\title{
APPLICATION OF NEURAL NETWORKS FOR ROUTING IN TELECOMMUNICATIONS NETWORKS
}

\author{
by
}

Mark Collett

\begin{abstract}
A thesis
presented to the University of Manitoba

in partial fulfillment of the requirements

for the degree of

Master of Science of Electrical Engineering

Department of

Electrical and Computer Engineering

University of Manitoba

Winnipeg, Manitoba, Canada 1992
\end{abstract}

(C) Mark Collett 1992 


\section{APPLICATION OF NEURAL NETWORKS FOR ROUTING}

IN TELECOMMUNICATIONS NETWORKS

BY

MARK COLLETT

A Thesis submitted to the Faculty of Graduate Studies of the University of Manitoba in partial fulfillment of the requirements for the degree of

MASTER OF SCIENCE

(c) 1992

Permission has been granted to the LIBRARY OF IHE UNTYERSITY OF MANITOBA to lend or sell copies of this thesis, to the NATIONAL LIBRARY OF CANADA to microfilm this thesis and to lend or sell copies of the film, and UNTVERSITY MICROFILMS to publish an abstract of this thesis.

The author reserves other publication rights, and neither the thesis nor extensive extracts from it may be printed or otherwise reproduced without the author's permission. 
I hereby declare that I am the sole author of this thesis.

I authorize the University of Manitoba to lend this thesis to other institutions or individuals for the purpose of scholarly research.

I authorize the University of Manitoba to reproduce this thesis by photocopying or other means, in total or in part, at the request of other institutions or individuals for the purpose of scholarly research. 


\begin{abstract}
This thesis applies neural networks to an application in the field of telecommunications. Neural networks are investigated to determine if they can be used to perform routing decisions within a telecommunications network. Once a neural network was modelled to perform the routing function for an individual node in a network, the modelling is extended to a sample telecommunications network to evaluate its performance in a variety of network conditions.
\end{abstract}




\section{Acknowledgements}

I would like to thank Dr. Witold Pedrycz for his guidance and support in the production of this thesis, and Matt Jensen - the creator of the MacBrain program - for his advice in using MacBrain to perform the neural network simulations performed as part of the research. 


\section{Dedication}

This thesis is dedicated to

Jackie, for her constant support and interest in this work My parents, for their constant interest and inquiries, and Shatzie, who always reminded me when it was time to take a break. 


\section{Table of Contents}

Chapter 1 - Introduction

1.1 Purpose

1.2 Problem 1

1.3 Scope 2

\section{Chapter 2 - Overview}

2.1 The telecommunications network 4

2.1.1 Telecommunications network structure 5

2.1.2 Routing and network reliability 8

2.1.3 Transport network protection and restoration 11

2 1.3.1 Protection channels and manual restoration 11

2.1.3.2 Digital cross connect systems 14

2.1.3.3 Survivable ring architectures 14

2.1.3.4 Dynamic restoration methods and self healing $\quad 17$

2.2 Routing Theory 20

2.2.1 Combinatorial techniques 22

2.2.2 Algebraic techniques 24

2.3 Neural networks and telecommunications routing 24

2.4 Attributes of an optimal routing method using neural networks 27

2.5 Description of the routing method under study 28

Chapter 3 - Single node modelling

3.1 Description 33

3.1.1 Description of the sample network 33

3.1.2 Description of the neural network 34

3.1.2.1 Learning rule 36

3.1.2.2 Topology 36

3.1.2.3 Activation function and associated variables 36

3.1.2.4 Learning rate and momentum 37

3.1.3 Determination of the routing information 38

3.1.4 Description of the modelling package 43

3.2 Training the neural networks 46 
3.2.1 The V1 Macro 46

3.2.2 Initial analysis of the V1 Macro trained networks 47

3.2.3 The V2 and V3 Macros 53

3.3 Analysis of the V1, V2, and V3 Macro results 56

3.3.1 Analysis of the individual network results. 56

3.3.1.1 Average and standard deviation results for the trained networks 57

3.3.1.2 Full and modified value results $\quad 57$

3.3.1.3 Distribution of difference values $\quad 59$

3.3.1.4 Direct comparison of results between the different networks and macros $\quad 60$

3.3.2 Comparison \#1 - description and results 60

3.3.3 Comparison \#2 - description and results 61

3.3.4 Comparison \#3-description and results 64

3.3.5 Comparison \#4 - description and results 64

3.3.6 Summary of conclusions from Comparisons 1 to $4 \quad 67$

3.3.7 Comparison of the routing results of the trained networks 68

$\begin{array}{ll}\text { 3.3.8 Modification of the training set } & 72\end{array}$

3.3.9 Comparison of the initial and modified training set networks 75

3.4 Conclusions from single network node modelling 77

\section{Chapter 4 - Full network modelling}

4.1 Full network simulation results 80

4.2 Performing routing decisions 81

4.2.1 Postprocessing of the output values 83

4.3 Simulation of a routing decision - normal network 87

4.3.1 Successful selection of optimal route 87

4.3.2 Unsuccessful selection of optimal route 89

4.4 Simulation of a routing decision around unavailable links 91

4.4.1 Selection of an alternate route to the destination node 92

4.4.2 Selection or alternate routing around an unavailable link 93

4.4.3 Unsuccessful selection of alternate route 96

4.5 Simulation of a routing decision - node failure 97

4.5.1 Routing around an unavailable node between originating and destination nodes

98 
4.5.2 Routing determination when the destination node is $\begin{array}{ll}\text { unavailable } & 101\end{array}$

$\begin{array}{ll}\text { 4.5.3 Unsuccessful selection of alternate route } & 102\end{array}$

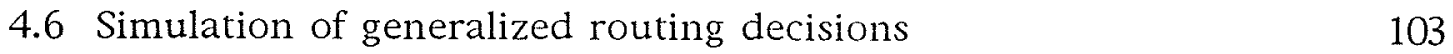

$\begin{array}{ll}\text { 4.6.1 Generalization test results } & 105\end{array}$

$\begin{array}{ll}\text { 4.6.2 Summary of generalization tests } & 109\end{array}$

Chapter 5 - Conclusions and recommendations

$\begin{array}{ll}5.1 \text { Conclusions } & 110\end{array}$

5.2 Recommendations 111

References

Appendix A - Training macros and sets

Appendix B - Single node modelling results

Appendix C - Full network modelling results 


\section{List of Figures}

Figure number and title Page

Figure 2.1: Sections of a telecommunications network 6

Figure 2.2: Analog and digital telecommunications network 8

Figure 2.3: 1:1 and 1:N protection $\quad 12$

Figure 2.4: Manual rerouting of traffic around a failed facility 13

Figure 2.5: Digital cross connect system 15

Figure 2.6: Fast facility protection 16

Figure 2.7: Fibre Distributed Data Interface 18

Figure 2.8: Shared protection ring 19

Figure 2.9: Method of routing under study 30

Figure 3.1: Sample network 33

Figure 3.2: Neural network and its relation to the sample network 35

Figure 3.3: Comparison of sample network to a geographic area of equivalent scale 41

Figure 3.4: Neural network with 16 input neurons, 6 output neurons, and 2 hidden layers of 6 neurons each 45

Figure 3.5: Neural network with 16 input neurons, 6 output neurons, and 2 hidden layers of 8 neurons each 45

Figure 3.6: Neural network with 16 input neurons, 6 output neurons, and 2 hidden layers of 10 neurons each 45

Figure 3.7: Flowchart for the V1 Macro 48

Figure 3.8: MSE Values as a function of number of training cycles for network V1.2.06.4

Figure 3.9: MSE values for networks with two output neurons using the V1 Macro 50

Figure 3.10: MSE values for networks with four output neurons using the V1 Macro

Figure 3.11: MSE values for networks with six output neurons using the V1 Macro

Figure 3.12: MSE values for networks with six neurons per hidden layer using the Vl Macro

Figure 3.13: MSE values for networks with eight neurons per hidden layer using the V1 Macro

Figure 3.14: MSE values for networks with ten neurons per hidden layer using the V1 Macro 
Figure 3.15: Comparison of MSE Values for V1 and Special Macros 53

Figure 3.16: Flowchart of the V2 Macro 54

Figure 4.1: Winner-take-all circuit 85

Figure 4.2: Final neural network topology 86

Figure 4.3: Routes from Node 6 to Node 13 through available 7 adjacent links $\quad 87$

Figure 4.4: Unsuccessful selection of optimal route from Node 11 to Node $14 \quad 90$

Figure 4.5: Alternate routes determined as a function of unavailable link (Routing from Node 16 to Node 3) 94

Figure 4.6: Alternate routes determined as a function of unavailable link (Routing from Node 3 to Node 16) 95

Figure 4.7: Alternate routes around unavailable links for each unavailable link

Figure 4.8: Alternate routing around unavailable node to destination node 99

Figure 4.9: Alternate routing around an unavailable node 100

Figure 4.10: Attempt to determine alternate routing for an unavailable destination node 101

Figure 4.11: Generalized routing examples 104 


\section{List of Tables}

Table number and title Page

Table 3.1: Valence values for the sample network 34

Table 3.2: Length values for each link in the sample network 40

Table 3.3: Scaled node and link weights and final network lengths 42

Table 3.4: Pattern file for Node 2 of the sample network 44

Table 3.5: Neural network topologies used in the initial testing 46

Table 3.6: V1 Macro minimum MSE results and number of training cycles 52

Table 3.7: Comparison of MSE results for the V1, V2, and V3 Macros 56

Table 3.8: Training results for V1.2.06.4 (Network 2.06.4, V1 Macro) 58

Table 3.9: Comparison \#1: Average/standard deviation measurements and distribution counts 62

Table 3.10: Comparison \#2: Average/standard deviation measurements and distribution counts 63

Table 3.11: Comparison \#3: Direct comparison of difference values 65

Table 3.12: Comparison \#4: Direct comparison of difference values 66

Table 3.13: Comparison of target rankings to trained rankings of 2 output neuron networks using the $\mathrm{V} 2$ Macro

69

Table 3.14: Comparison of target rankings to trained rankings of 4 output neuron networks using the V2 Macro

Table 3.15: Comparison of target rankings to trained rankings of 6 output neuron networks using the V2 Macro

Table 3.16: Comparison of routing performance versus number of neurons per hidden layer 72

Table 3.17: Quantized output values for different values of $\mathrm{n} \quad 74$

Table 3.18: Comparison of MSE results for networks using original and modified training sets

Table 3.19: Neural Network Model Test Results: Average/standard deviation measurements and distribution counts Comparison of V2 trained networks using real and modified training sets

Table 3.20: V2 Macro 2 output neuron routing comparison - real vs. modified training set

Table 3.21: V2 Macro 4 output neuron routing comparison - real vs. modified training set 
Table 3.22: V2 Macro 6 output neuron routing comparison - real vs. modified training set

Table 4.1: Routing results for node 12 of the sample network

Table 4.2: Comparison of routing performance for Sample Network for entire network and different valence values

Table 4.3: Test \#1: Route selection from Node 13 to Nodes 6 and $14 \quad 107$

Table 4.4: Test \#2: Route selection from Node 5 to Nodes 13 and $14 \quad 107$

Table 4.5: Test \#3: Route selection from Node 8 to Nodes 6 and $12 \quad 107$

Table 4.6: Test \#4: Route selection from Node 16 to Nodes 4 and $6 \quad 108$

Table 4.7: Test \#5: Route selection from Node 4 to Nodes 10,12 and $16 \quad 108$

Table 4.8: Test \#6: Route selection from Node 3 to Nodes 9, 14 and $15 \quad 108$ 


\section{Chapter 1}

\section{Introduction}

\subsection{Purpose}

The purpose of this thesis is to research the application of neural networks to a specific issue in the field of telecommunications. The issue being considered is the problem of performing efficient shortest path routing decisions within a telecommunications network under normal and abnormal conditions.

The study of neural networks has been a steadily increasing area of interest over the past 5 years, where the application of neural networks to different scientific and engineering disciplines has produced alternative methods to solving classic problems [1]. Neural networks have been applied to areas of optimization, control, associative memories, and pattern recognition [2], areas that can be applied to routing in telecommunications networks. By studying the concept of routing both from the specific field of telecommunications and the more general field of routing theory, this thesis will map routing concepts into the neural network paradigm, define a neural network solution, and demonstrate through modelling the ability of a neural network to perform telecommunications network routing.

\subsection{Problem}

The operation of the telecommunications network depends on the efficient use of the components in the network, and network routing is a key factor in determining this efficiency. Improper network routing results in poor network utilization, which in turn affects the services that the network can provide.

As telecommunications networks evolve, the importance of routing in these 
networks is increasing. This increased importance is due to a number of factors: telecommunications companies are under pressure to become more efficient, requiring maximum utilization of network components; the increased dependence of the public and corporations on these networks requires higher levels of network reliability and availability; and the introduction of new technology has made the network more complex. Routing methods must evolve to maximize network utilization under normal conditions, plus adapt to problems in the network so that the effects of any abnormal network condition is minimized to provide maximum reliability and availability.

A neural network router can be created that can meet these requirements. While new telecommunications routing methods are being researched, only a small portion of this work involves neural networks. Conventional approaches are superior to the older, manual routing methods, but still have disadvantages in terms of speed and flexibility. By utilizing the adaptive properties of neural networks, an advanced method of network routing could be created without the disadvantages of conventional routing methods.

\subsection{Scope}

This thesis is composed of six chapters. In Chapter 2, an overview of relevant information to this thesis is provided, and begins with an introduction to and description of telecommunications networks. After this general discussion the issue of network routing and reliability will be introduced. This is followed by a discussion of current and proposed routing methods within telecommunications networks, and a brief discussion of routing theory. The chapter concludes with a discussion on the application of neural networks in telecommunications, and a description of the specific routing approach to be studied. In Chapter 3, the modelling of a single node within a defined sample network will be presented, with the objective of optimizing the topology, learning, and neural network training set to perform the network routing task. The results of Chapter 3 will be applied in Chapter 4 , where the routing for the full sample network will be modelled, the results discussed, and the 
performance of the neural networks evaluated for different routing conditions. Chapter 5 contains conclusions on the research and recommendations on areas for further investigation. The thesis concludes with a list of references used in this research, and appendices containing related material. 


\section{Chapter 2}

\section{Overview}

This chapter will provide an overview of topics of interest in this thesis. The chapter will first review of the operation of telecommunications networks, then discuss the nature and current methods of routing within these networks. A review of methods and research in the area of routing theory is then provided, and this is followed by a discussion of current applications of neural networks to telecommunications routing. The desired attributes for a neural network router are then outlined, and the method of routing studied in the remainder of the thesis is discussed.

\subsection{The telecommunications network}

The telecommunications network provides services that most people take for granted. To the majority of people, telecommunications is represented by the telephone, and the process of inputting a series of numbers into the telephone that then connects the calling person to another telephone. But between these telephones is a massive investment by utilities and corporations in a complex telecommunications network. This network provides not only the basic connection service familiar to the public, but also specialized services to corporations that rely on the network for providing service to their customers. These specialized services are becoming more important as telecommunications networks evolve, and are projected to become an important factor in the future competitiveness of many companies and countries.

As stated above, the telecommunications network is a complex system. Providers of telecommunications services - typically the phone company must plan, install, and maintain these networks. Underlying these network activities is the requirement to provide a high level of service to its users. In some areas of telecommunications a monopoly exists, and a single network 
provider is regulated by a government to provide service at a price that allows for reasonable rates of return on the provider's investment. In other areas telecommunications services are deregulated, and multiple service providers compete in the marketplace to provide services based on the cost of the network and the variety of services. In Canada, an example of a service monopoly is long distance telephone service, and an example of a deregulated service is cellular phone service. The global trend in telecommunications is toward deregulation; as will be discussed later, this results in the need for increased efficiency, reliability, and functionality within the network, and the need to find new methods of controlling and managing this network.

\subsubsection{Telecommunications network structure}

The basic telecommunications network shown in Figure 2.1 is composed of three major sections, with each section performing a specific network function. The first section is called the access section; this section connects the equipment of a telephone user - known as customer premise equipment to the telephone switching center or central office. The customer premise equipment is connected by a dedicated set of wires or access lines to the central office. This configuration is the most simple example of the access portion of the network, but there are other configurations. One example is where a switching element similar in function to a central office is placed between a central office and the customer premise equipment. In one example, a switching element is located at the business site, and is called a private branch exchange, or PBX. A second example is where a smaller switching center, called a remote, is placed to service a portion of a central office's serving area. The purpose of both the PBX and the remote is to concentrate a large number of access lines into a smaller number of transport facilities; this will be described in the following paragraphs.

The second section of a telecommunications network is the switching section, where a central office concentrates the access lines of a specific geographic area and connects these lines to access lines in its own area or to transport facilities between it and central of fices in other areas. To provide full 


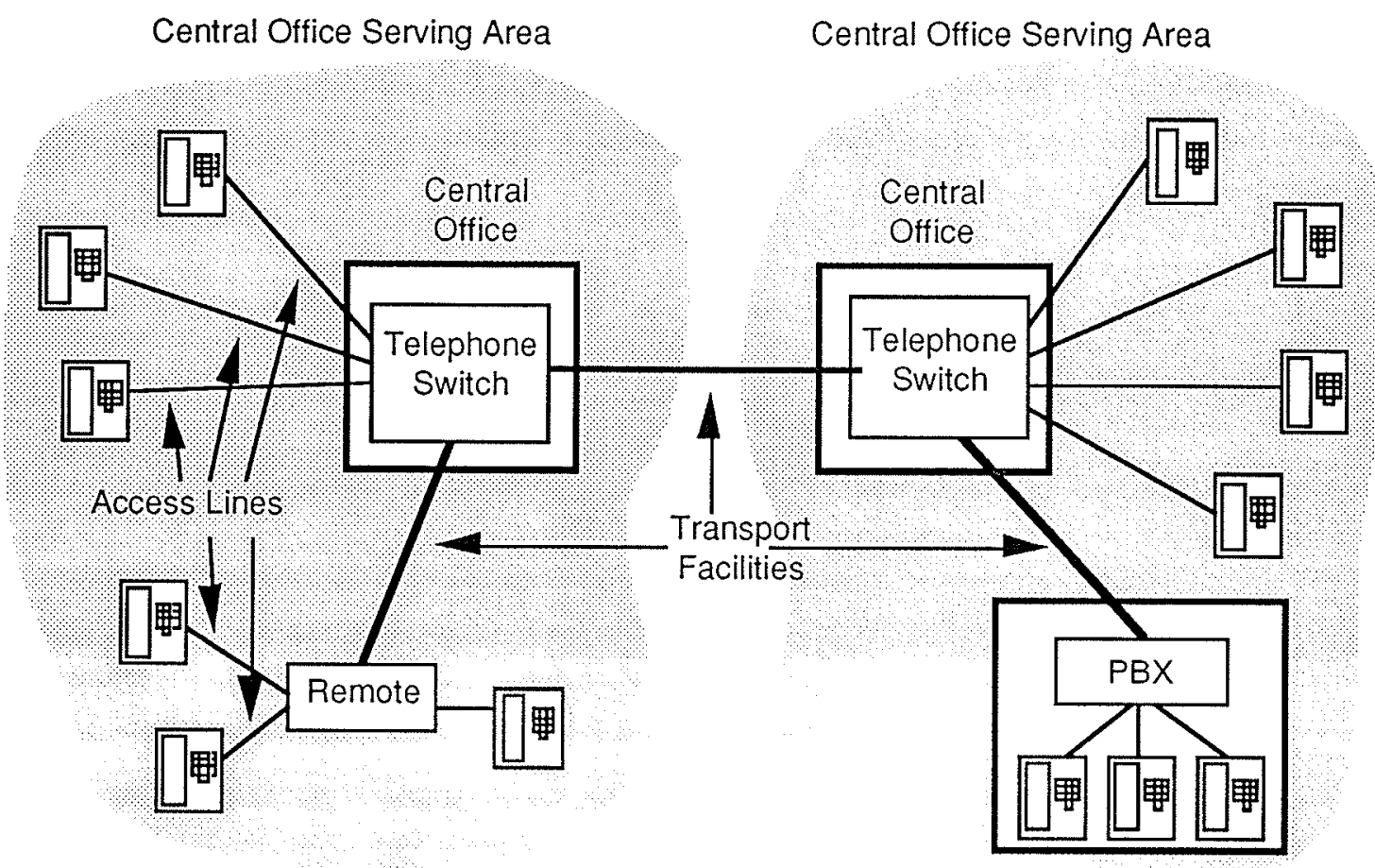

Figure 2.1:

Sections of a Telecommunications Network

interconnection between the hundreds of millions of telephones in the telecommunications network is not possible; instead the network relies on the fact that only a small portion of the telephones in the network are in use at a given time to reduce the number of connections required. The main component of the central office is the telephone switch, which is connected to all access lines in its geographic area and then selectively connects the lines in use to the access line of a telephone that is being called. In the case where the called telephone is within the same geographic area as the calling telephone, the telephone switch will connect them within the central office. If, however, the called telephone is in a geographic area served by another telephone switch, then some form of link is required between these switches. This link is called a transport facility, and is part of the set of dedicated interoffice links that forms the transport portion of the network. Depending on the location of the destination telephone switch, a series of telephone switches and transport facilities may be required to provide the end-to-end connection. The location of the called telephone and telephone switch is determined from the telephone number, which can be considered to be a 
network address.

The final section of the network is the transport section. As stated above, transport facilities are dedicated links between central offices, and like the facilities in the access section of the network, the links are not all in use at one time. The amount of activity on a specific transport facility is a function of the network traffic between the central offices, and a properly planned transport facility will have the available capacity to handle the heaviest traffic requirements under the busiest, or peak, conditions. When the traffic between two offices reaches the level where the transport facility is at full capacity, no further connections can be provided over that link.

Advances in technology have produced dramatic changes in telecommunications networks. One of the most dramatic revolutions has been the migration of the network from analog to digital systems. In the analog network shown in Figure 2.2(a), the access, switching, and transport sections were analog based; an analog signal originated from the telephone, connected with a physical connection at the calling telephone switch to an analog transport facility, and connected through a physical connection at the destination telephone switch to the called telephone. With the evolution to digital technology shown in Figure 2.2(b), only the access section of the network remains analog; at the digital switch, the analog signal is digitized, and the digital signal is now digitally switched and carried over digital transport facilities to a destination digital switch where is it converted to analog for connection to the called telephone. The benefits of the digital network are significant: noise has been reduced, improved reliability has been achieved, and the performance and capacity of the network has been increased. One of the major benefits of digitization of the transport facilities is the use of time division multiplexing to combine multiple low speed signals from the access network onto a single high speed transport facility. While an analog transport facility consisted of thousands of wire pairs with each pair representing a single transport circuit, a single digital transport facility can now carry thousands of time multiplexed channels, with each channel representing a transport circuit. The introduction of fibre optics transmission technology in the telecommunications network has provided the most 


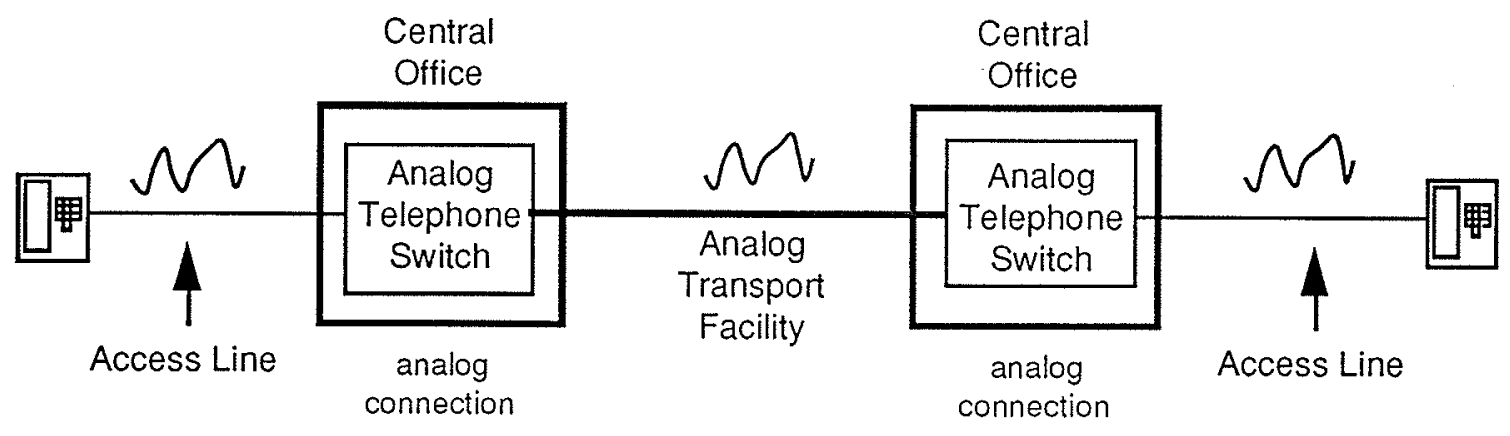

Figure 2.2(a):

Analog Telecommunications Network

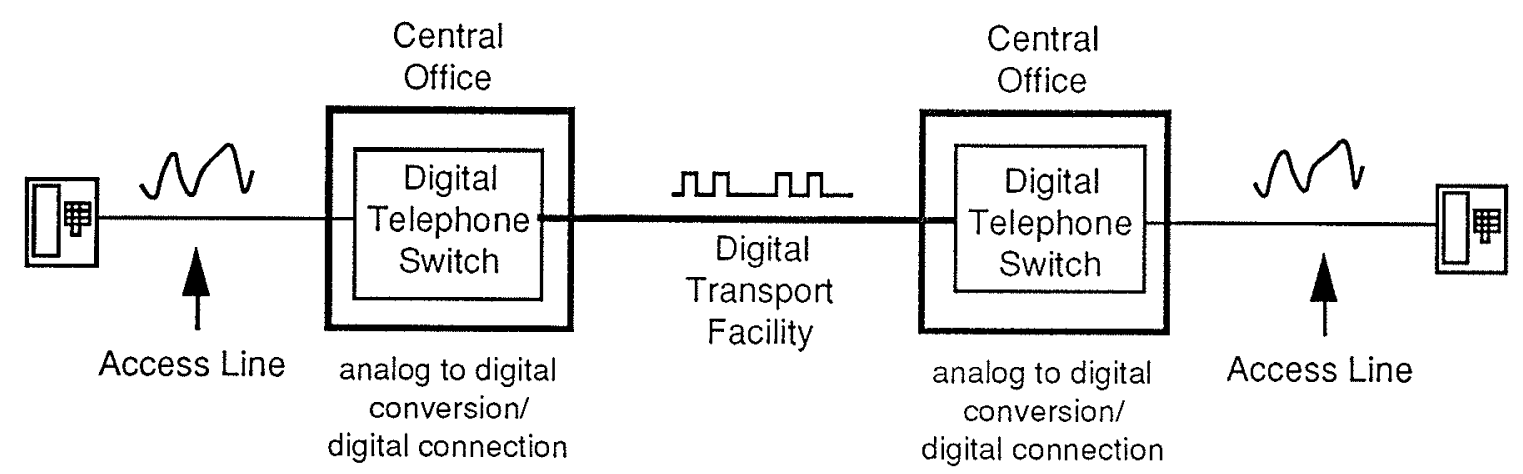

Figure 2.2(b):

Digital Telecommunications Network

significant increases in capacity, with new systems now providing over 30000 voice channels, or a combination of voice, data, and video signals, on one fibre optic pair.

\subsubsection{Routing and network reliability}

To obtain maximum efficiency from a telecommunications network, digital signals representing voice, data, or video signals must be routed between the originating and destination points in the network quickly and reliably.

Routing activity occurs at several points between the originating and destination locations. First, the telephone switch translates the telephone number into routing information, and the connection is routed through the switch to another access line or a transport facility. If the destination is within the switch's serving area, the routing is performed within that switch. 
If the destination is in another central office, then a destination telephone switch will route the call from the transport facility that connects it to the originating central office to the access line of the destination telephone. As the distance between originating and destination central offices increases, more transport facilities and telephone switches may be required to provide the end-to-end connection, with each switch performing similar routing functions. Under normal traffic conditions the network performs this activity with a high level of reliability. If, however, an abnormal condition occurs, then the network attempts to compensate for the condition, and depending on the type of condition, different compensation methods can be used. If a transport facility reaches capacity, the telephone switch can redirect traffic to other transport facilities along other routes that have not reached capacity. But if a transport facility becomes unavailable as a result of failure, the transport network must attempt to restore the lost capacity by using spare capacity in the network. The ability of the network to route traffic under normal and abnormal conditions is a fundamental requirement.

One of the most important issues in the telecommunications network is network reliability. Some of the reasons are:

- Network providers are under increasing pressure to provide new services. These new services increasingly rely on information stored in the local and remote computer systems of network providers, resulting in increased network communication.

- Some new services are data applications. These applications require higher bandwidths and lower bit error rates than regular voice transmission.

- Digital technology has allowed for increased capacity within the network; as a result, more services and users are affected when a network problem occurs.

- While the amount of required maintenance and been reduced and the overall availability of network equipment has been improved, the problems that now occur in the networks are becoming increasingly more complex.

- Network failures can be caused by a variety of factors, including human error, natural disasters, terrorism, and the complexity of 
modern equipment used in the digital network.

- The reliance of the users on services and the introduction of service competition has resulted in the need for increased reliability and availability in the network.

- Though rare, major network failures can affect thousands of users and disrupt activities over a large area. In one recent event, a telephone switch in New York City failed; tens of thousands of users lost basic communications, financial operations were affected and air traffic control was disrupted in regional airports [3]. In the worst case, the financial cost can be significant; a fire in a central office in Chicago disrupted operations at O'Hare airport and resulted in service outages of up to a month for some residential and business customers; the estimates of the cost of lost business range from the hundreds of millions to billions of dollars [4].

To mitigate the effect of future failures, network providers are deploying systems that provide improved network routing, restoration and survivability. Depending on the section of the network (access, switching, or transport) different systems or methods are available. Of the three sections of the network, this thesis will focus on the routing and restoration in the transport section. The reasons are:

- Transport failures are a frequent type of abnormal network condition.

- Transport failures affect more users across a larger geographic area.

- Since transport facilities are outside the controlled environment of central offices, they are more susceptible to failure from a number of areas.

- The topology of the transport network lends itself to a number of possible restoration solutions.

- The various restoration solutions all involve static or dynamic routing methods.

- If a routing method can be defined that can be mapped into a neural network structure, then a solution may be possible. 


\subsubsection{Transport network protection and restoration}

The areas of network protection and restoration have been extensively researched in telecommunications; a complete review of network protection and restoration methods is not required for this thesis. Instead, this section will provide a brief overview of existing and new methods.

\section{1.3.1 Protection channels and manual restoration}

The most widespread method of providing restoration of failed facilities is by the use of protection channels. A protection channel is a redundant or spare facility with the same capacity as the regular working facility it protects. Figure 2.3(a) shows a working and protection channel in normal operation, with the traffic being carried on the working channel. When a problem occurs with the working facility, the problem is detected and the traffic is switched by protection equipment to the protection channel, as shown in Figure 2.3(b). The method of protection where one working channel is protected by one protection channel is called 1:1 protection. A more general form of this protection is called $1: \mathrm{N}$ protection, where one protection channel provides a redundant facility for $\mathrm{N}$ working channels; if any one of the $\mathrm{N}$ working channels fails, the traffic can be restored on the protection channel. Figures $2.3(\mathrm{c})$ and $2.3(\mathrm{~d})$ depict the normal and restoration activities for a $1: \mathrm{N}$ system.

This form of restoration is well suited to facility failures that affect only one working channel of a transport facility; an example of this type of failure is where a portion of the transport equipment associated with one channel fails. Where this form of protection fails to perform is in instances where a failure affects an entire transport facility such as a failure of transport equipment common to all channels or the loss of the transmission medium such as the cutting of a fibre optic cable or loss of a microwave relay tower. In these cases, $1: 1$ and $1: \mathrm{N}$ protection methods cannot provide restoration, and restoration is performed by manually reconnecting the traffic from the failed facility onto the protection channels of one or more alternate transport facilities, restoring the traffic to the opposite end of the failed facility. This 


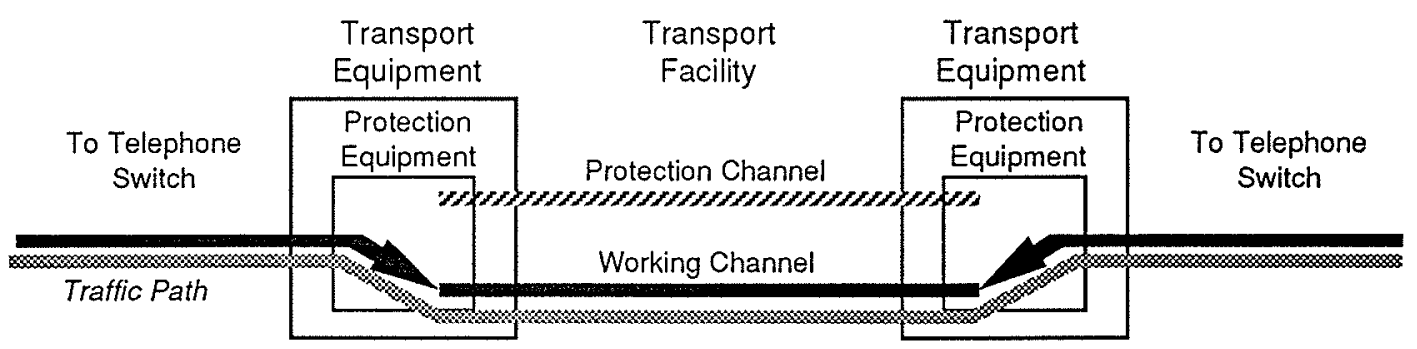

Figure 2.3(a): 1:1 Protection - Normal Condition

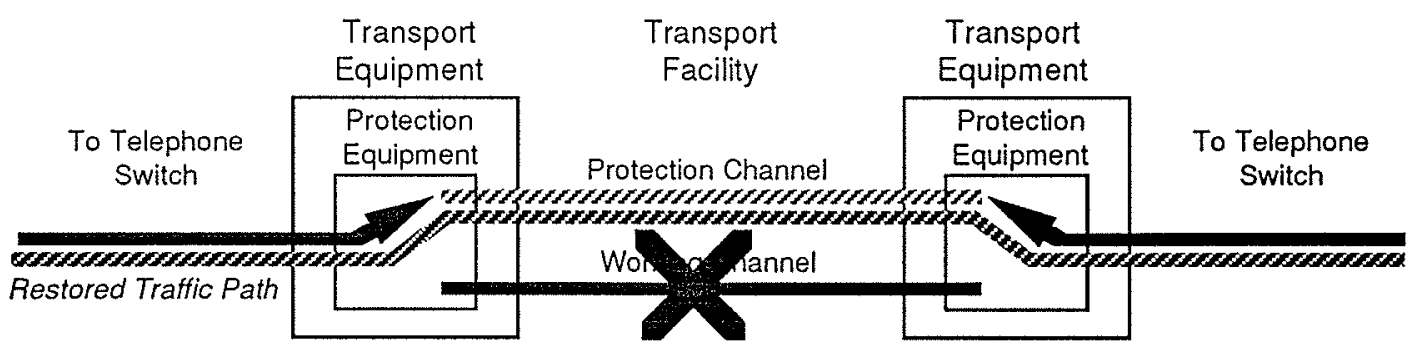

Figure 2.3(b): 1:1 Protection - Protection Condition

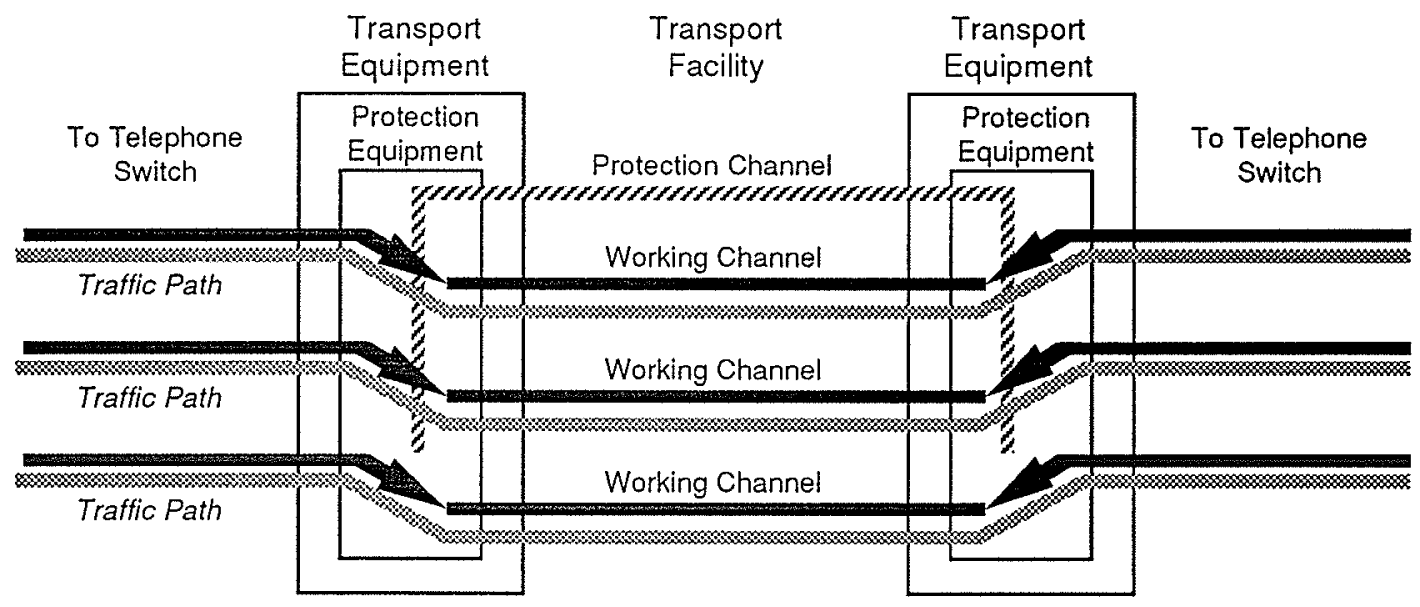

Figure 2.3(c): 1:N Protection-Normal Condition

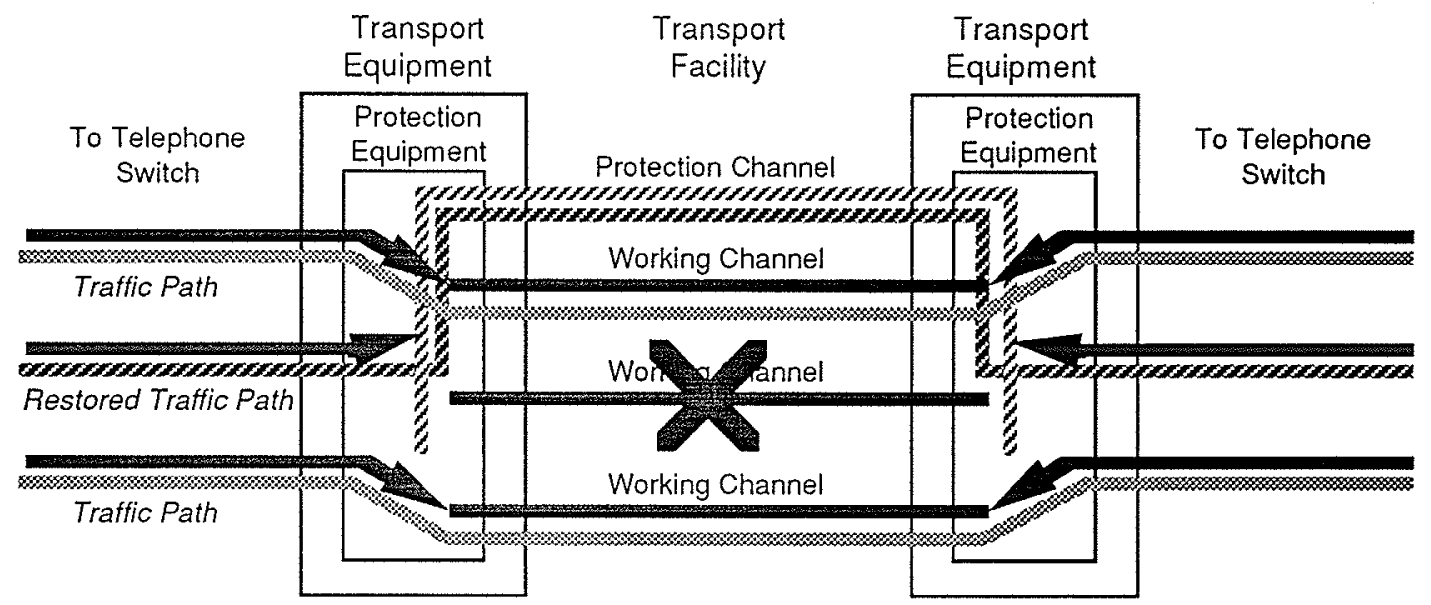

Figure 2.3(d): 1:N Protection - Protection Condition 


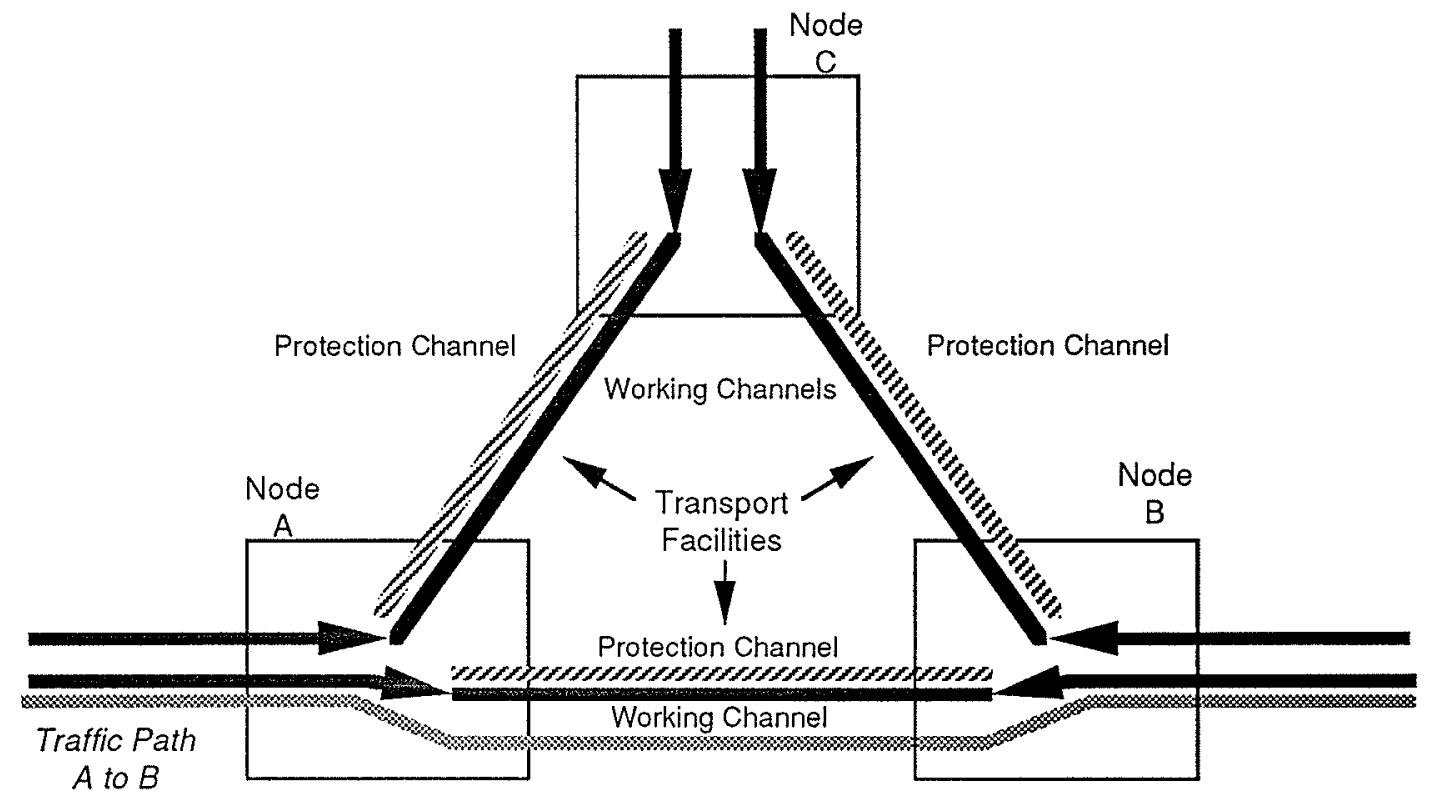

Figure 2.4(a):

Normal traffic conditions

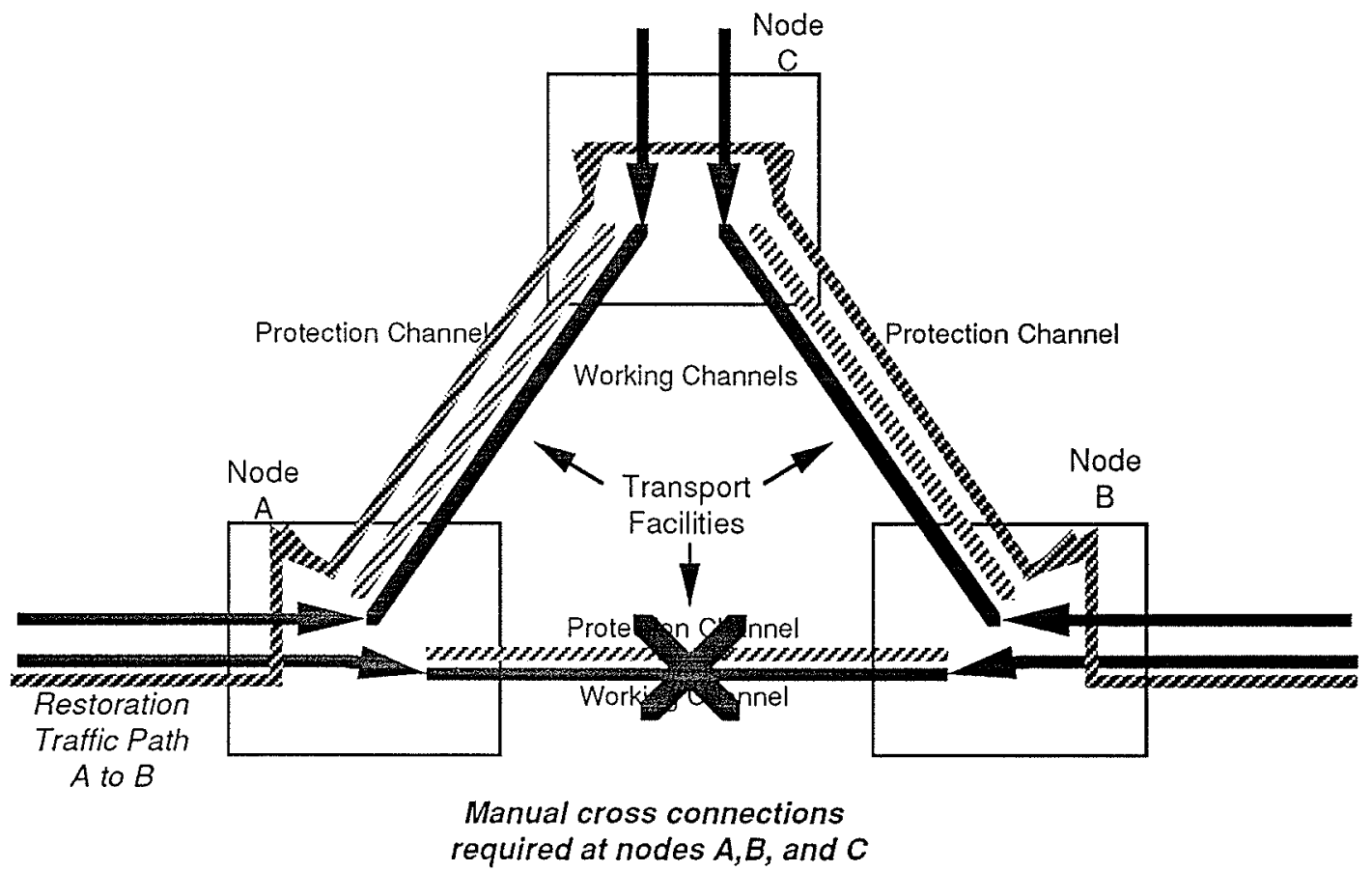

Figure 2.4(b):

Manual rerouting of a failed facility to an alternate traffic path 
process of manual rerouting is a time consuming process, and can result in the loss of service for significant periods of time; as a result, methods of providing faster restoration around a failed facility were needed. The process of manual rerouting of a failed facility is shown in Figure 2.4(a) and (b).

\subsubsection{Digital Cross Connect Systems}

The introduction of digital cross connect systems (or DCS) has been a significant development in the management of transport facilities. The DCS replaces the manual cross connect equipment in central offices, and provides remote and automatic control of the connections of digital facilities. The network provider operations staff can issue commands to a DCS to perform connections of different transport facilities, and in the case of the failure of a transport facility the operations staff can use a DCS to quickly perform the reconnections required to restore service. The placement and use of a DCS for restoration are shown in Figure 2.5.

The use of a DCS can reduce the time of restoration to minutes, but this still results in loss of traffic connections and service. To reach the goal of providing restoration without the loss of most traffic connections, the time to restore facilities must be in the 1-2 second range. The introduction of special features in the DCS is now making this level of restoration possible. Using a feature called Fast Facility Protection (FFP) [5], the protection channels for 1:1 protection systems can be routed over a different route to the destination. The traffic being carried is bridged onto both the working and protection channels by the DCS at one end of the facility, and at the other end the presence and signal quality of the traffic is monitored. If there is a loss of traffic (resulting from the loss of the entire facility), or if the signal quality is degraded, then the DCS at the other end switches to the protection channel. This feature is now available and is being considered for use by some network providers. An example of FFP is shown in Figure 2.6.

\subsubsection{Survivable Ring Architectures}




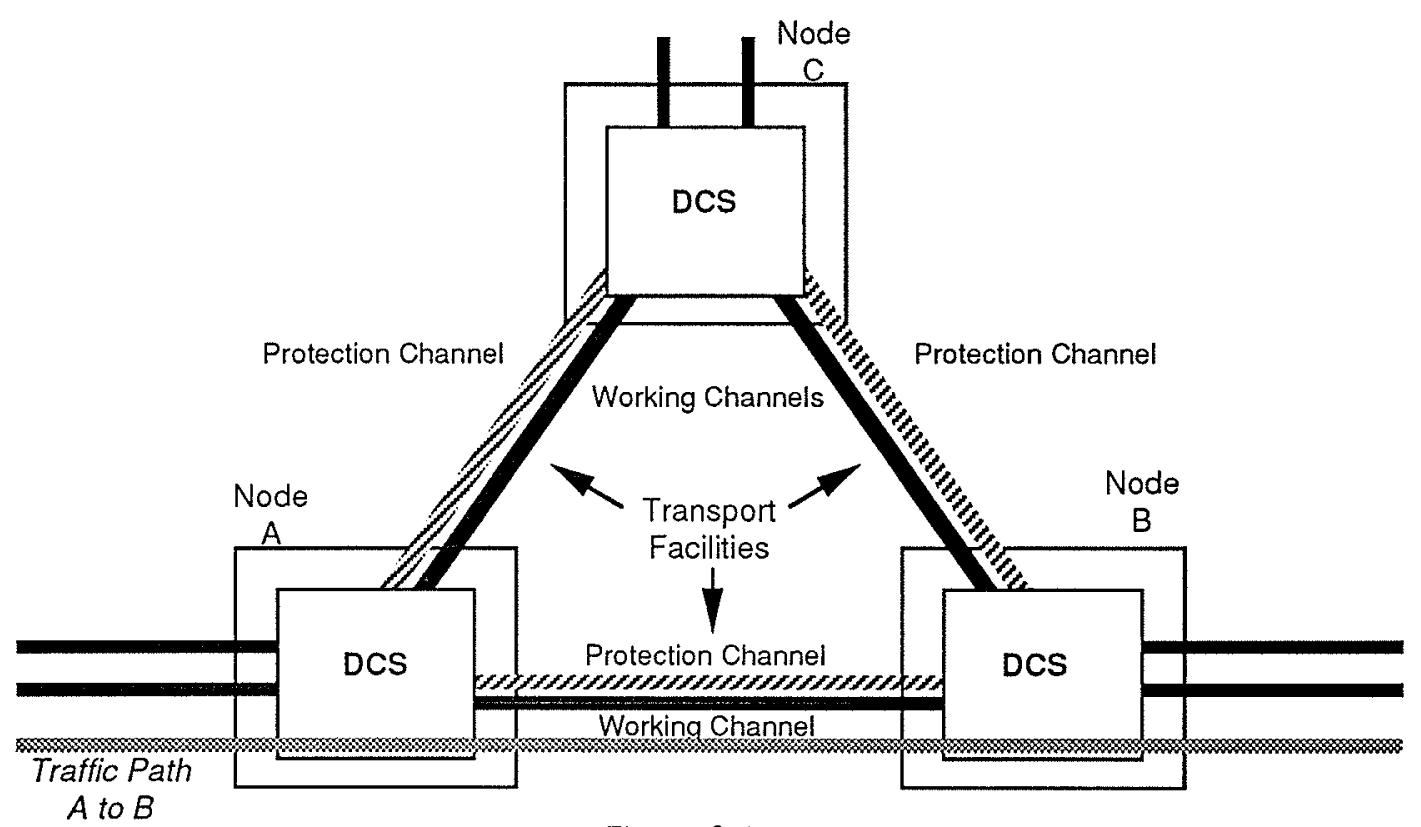

Figure 2.5(a):

Normal traffic condition with digital cross connects

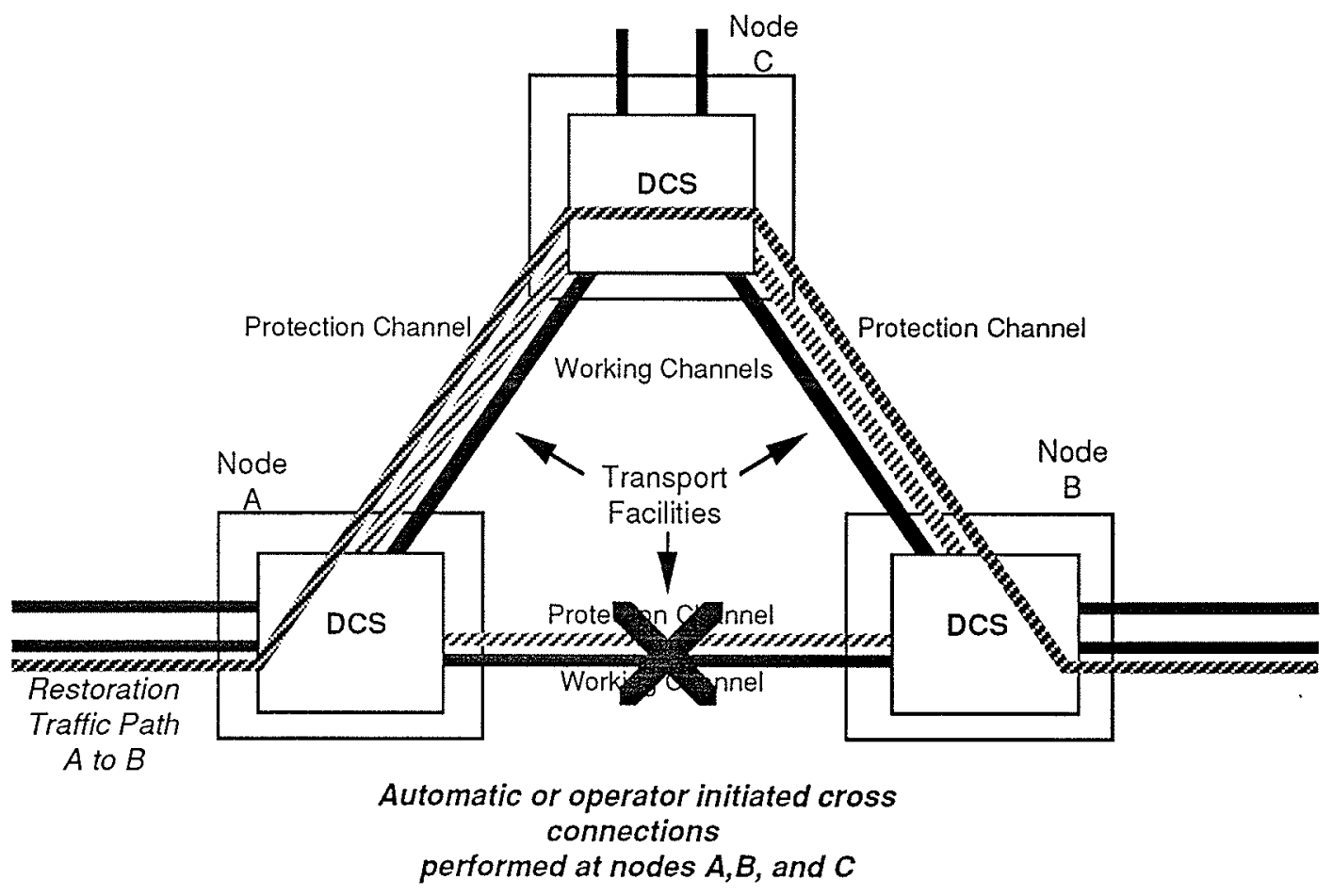

Figure 2.5(b):

Digital cross connect rerouting of traffic around a failed facility 


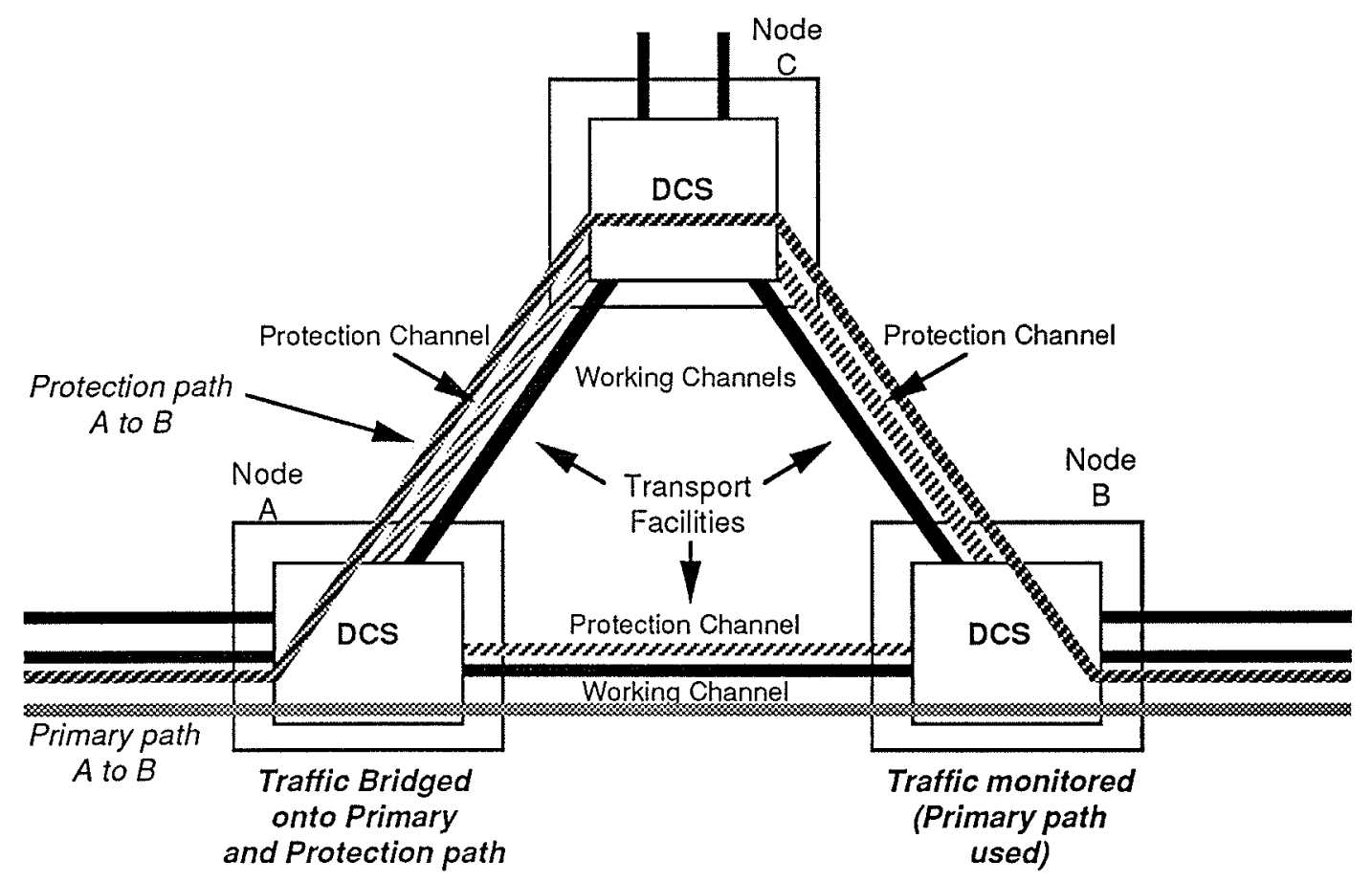

Figure 2.6(a):

Normal traffic condition with Fast Facility Protection

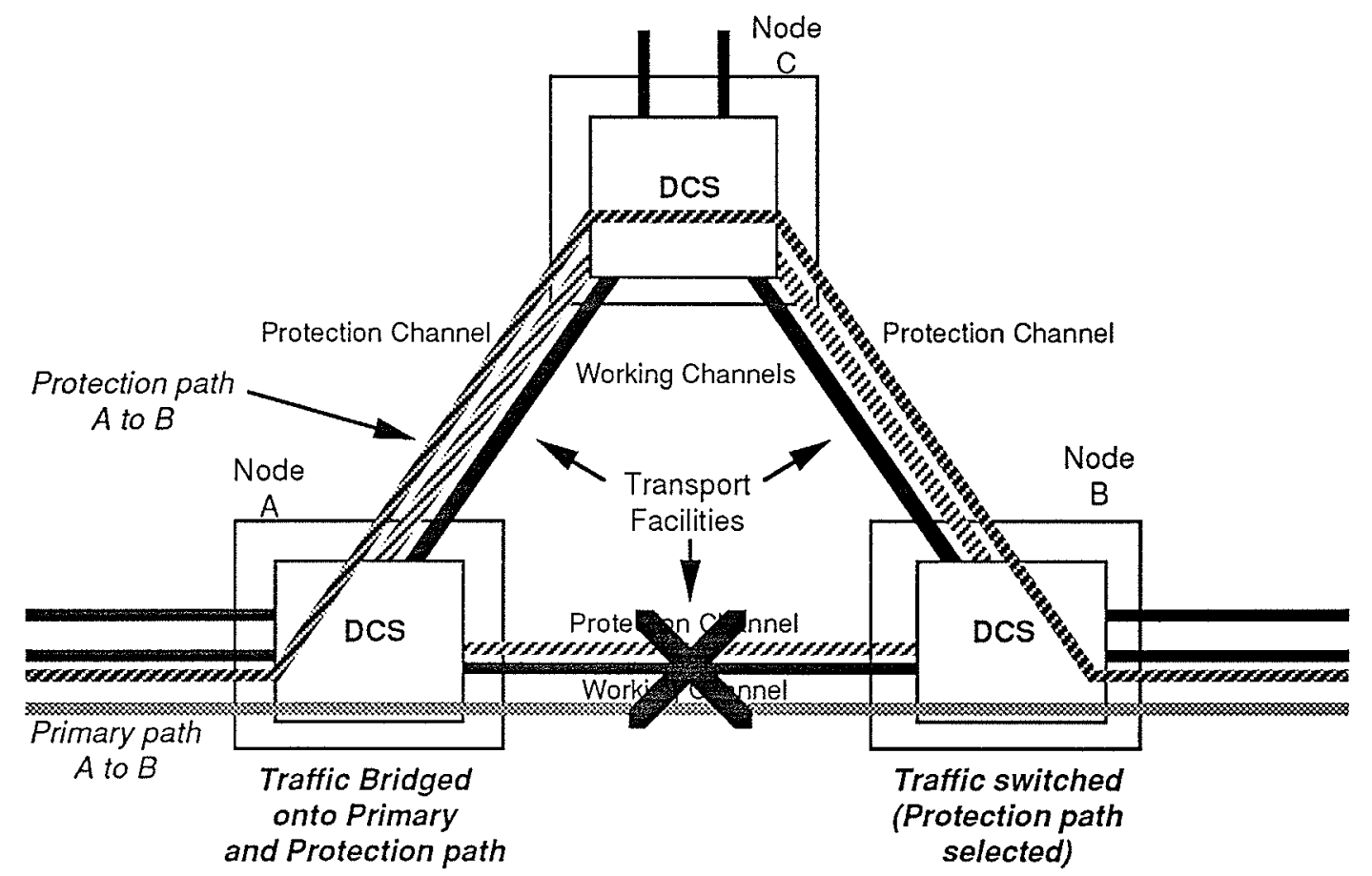

Figure 2.6(b):

Selection of alternate traffic path using Fast Facility Protection 
Another method of providing restoration is by implementing ring architectures in transport networks. By arranging the transport links between a number of separate locations into a ring, simple redundancy methods can be implemented. Current examples of this type of architecture are available from both the computer and telecommunications network areas.

For computer networks, the Fibre Distributed Data Interchange (FDDI) standard describes a 100 million bits per second fibre optic data network. To build survivability into the FDDI network, working and protection network traffic circulates around a fibre optic ring connecting all nodes, with the working and protection channels being transmitted in opposite directions around the ring. If any site on the loop experiences a failure, or the facility fails between two nodes, protection circuitry at the nodes detect the failure and redirect working traffic onto the protection channel, creating a single traffic loop connecting all nodes and isolating the failure. FDDI restoration is presented in Figure 2.7.

In telecommunications networks, one example of restoration using the ring architecture is provided by the Shared Protection Ring (SPRING) [6]. Like FDDI the SPRING has two traffic channels transmitting in opposite directions, but in the case of SPRING the working and protection capacity is split between the two rings, resulting in one half of the working traffic being transmitted in the opposite direction to the other half. If a facility failure occurs, the working capacity carried on the failed facility is redirected in to the protection capacity of the working facility, isolating the failed facility and maintaining traffic connections. SPRING restoration is presented in Figure 2.8 .

\subsubsection{Dynamic restoration methods and self healing}

Restoration provided by methods such as HP and ring architectures can provide rapid restoration from failures on the order of 50 milliseconds. This short restoration time will maintain traffic and service connections in the network, but there are drawbacks to these methods. Bandwidth must be dedicated to the restoration of specific facilities, resulting in the need to 

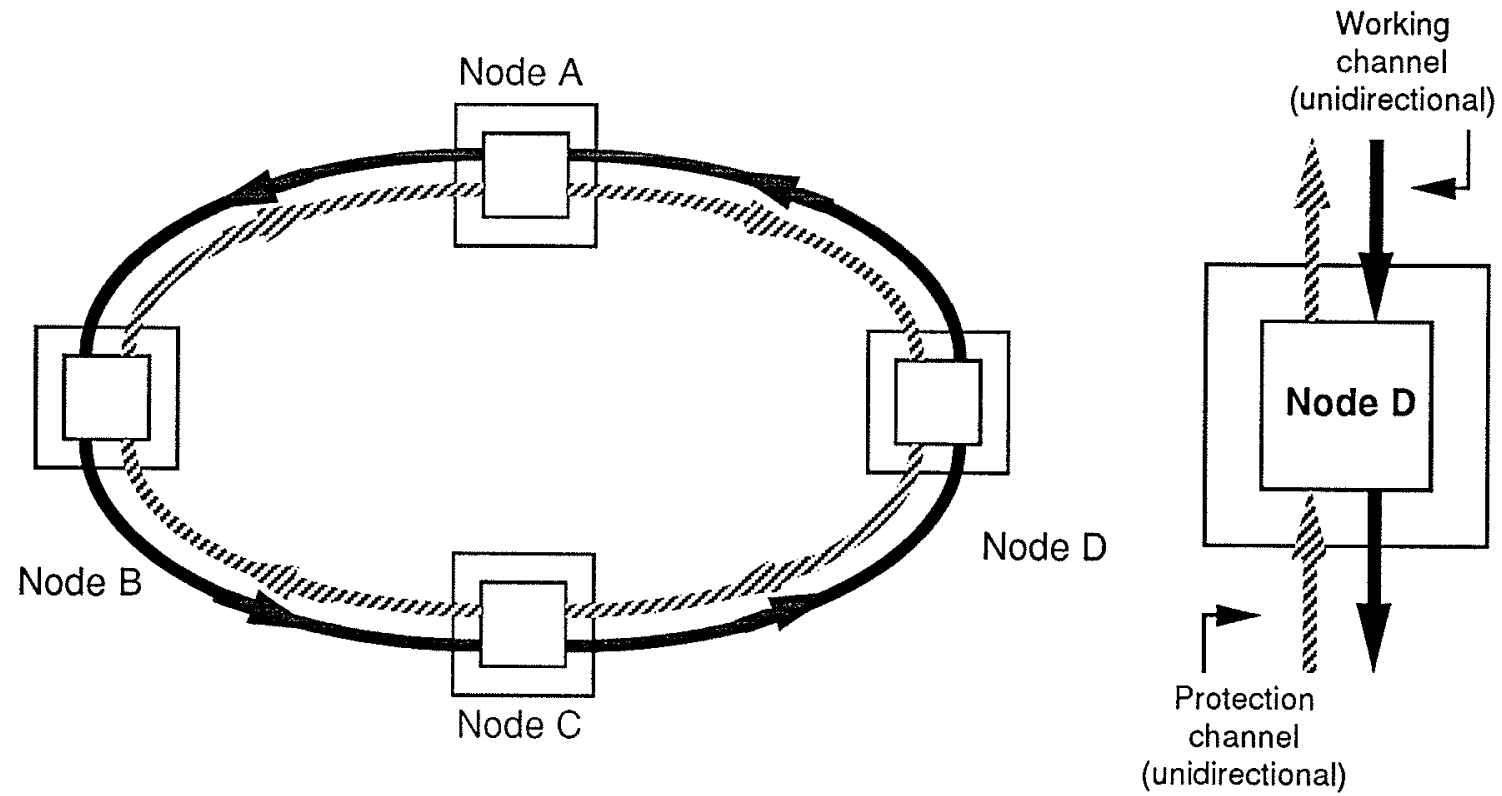

Figure 2.7(a):

Normal traffic on FDDI ring

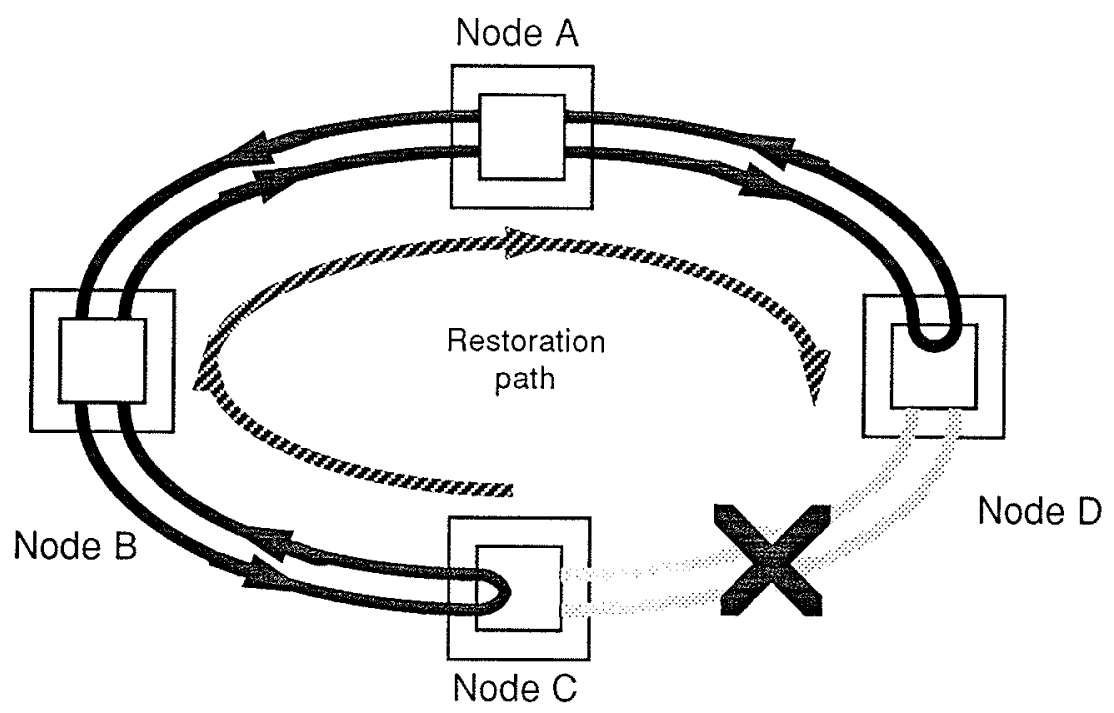

Working channel traffic looped back onto protection channel from

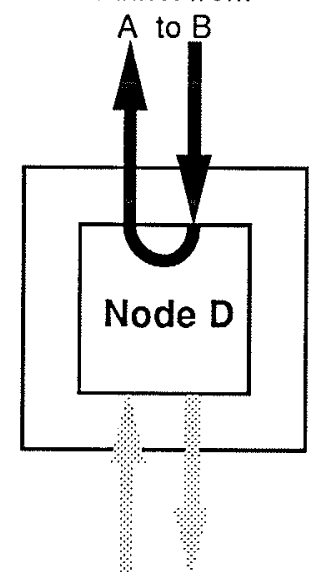

Working and protection channels lost between nodes $C$ and D

Figure 2.7(b):

Restoration of traffic on FDDI ring after loss of facilities between Nodes $C$ and $D$ 


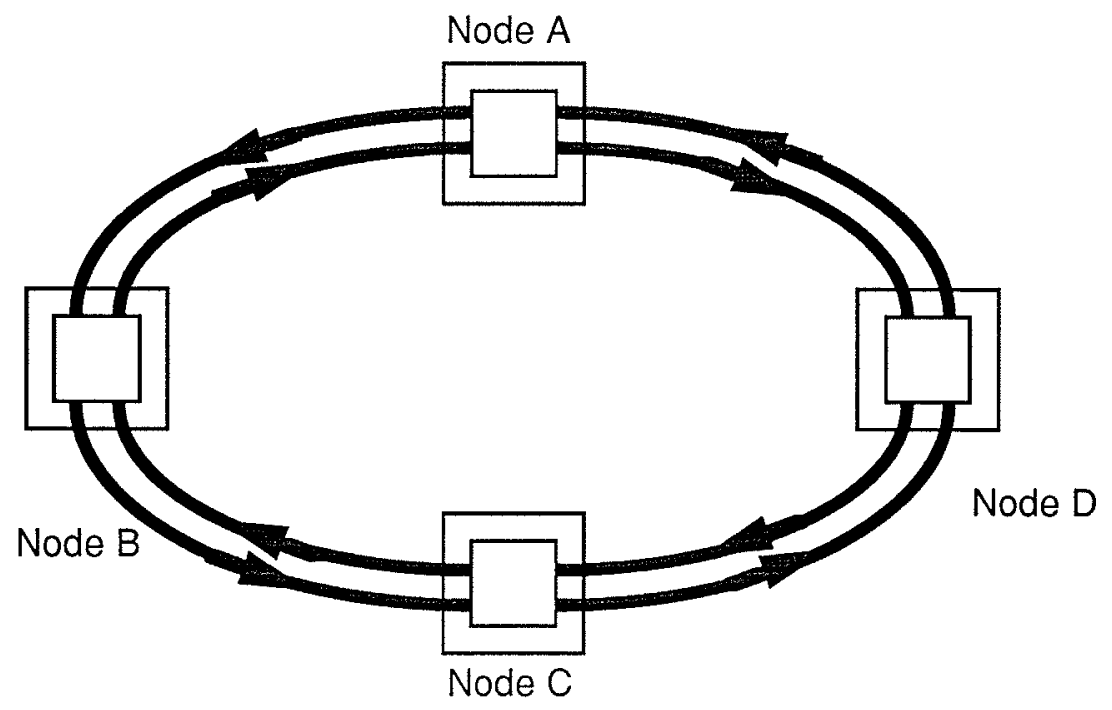

Figure 2.8(a):

Normal traffic on SPRING

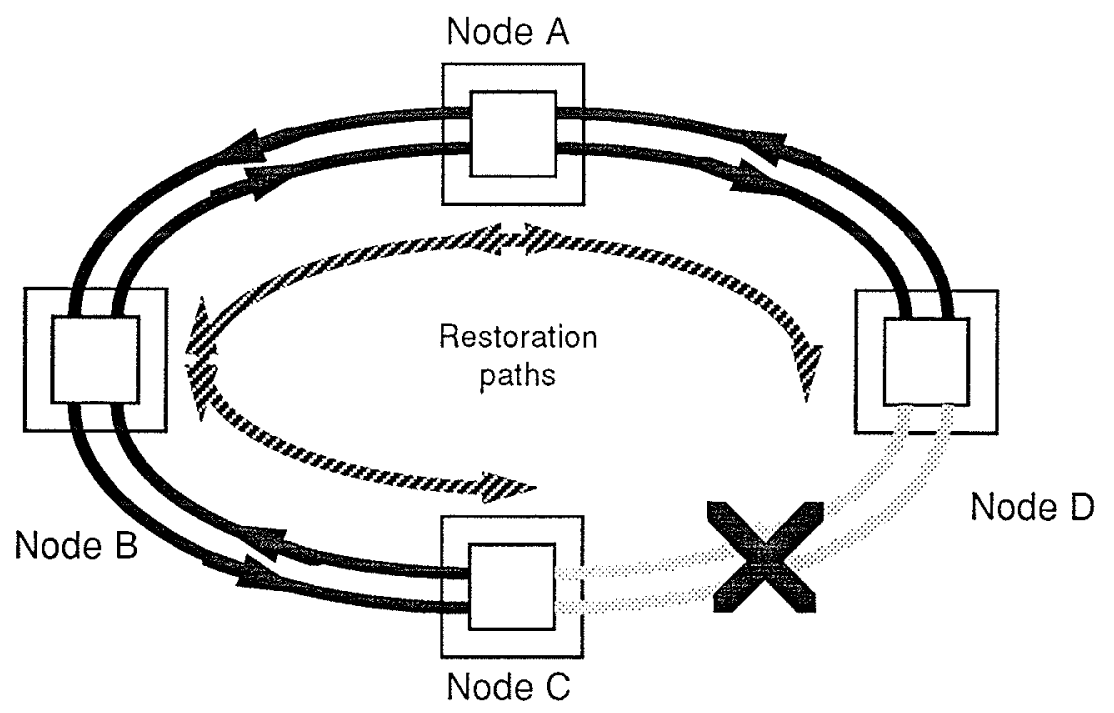

Working traffic from $C$ to $D$ is redirected to protection traffic capacity from

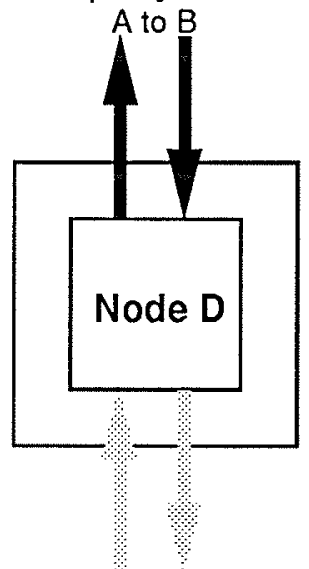

Working traffic lost between $\mathrm{C}$ and $\mathrm{D}$

Figure 2.8(b):

Restoration of traffic on SPRING after loss of facilities between Nodes C and D 
provision large amounts of channel capacity just for restoration and imposing added design rules on the network. In addition, these methods are based on static conditions in the transport network, and cannot adapt to multiple network changes should they occur. In order to provide flexibility and to reduce the need to provide large amounts of restoration capacity, several adaptive and dynamic restoration techniques have been proposed.

Dynamic restoration or self-healing techniques rely on the use of a restoration protocol implemented in transport network DCSs. When the failure of a transport facility occurs, a designated DCS sends restoration messages to adjacent DCSs over connecting facilities with available capacity. The process is repeated by these adjacent DCSs resulting in a "message wave" that floods the network. The target of this message wave - and the signature information contained in it - is the DCS at the other end of the failed facility; when the messages are received, the signatures are processed, and the alternate restoration routes and their capacities are determined. To complete the process, a return message is issued along the alternate routes to establish the restoration $\operatorname{link}(s)$ required to restore all traffic. This self-healing process has been suggested by Yang and Hasegawa [7] and Grover et al [8]: in the former case the authors based their approach on the implementation of specific types of equipment in the transport network; in the latter case, a more generalized form and more well defined protocol has been created. For both these methods, the restoration time has been estimated in the 1.5-2 second range.

\subsection{Routing Theory}

Routing has been researched extensively from a theoretical perspective to determine efficient algorithms; for the purposes of this thesis, the study was focussed on the area of shortest path (SP) problems. SP problems are encountered in many areas of research apart from communications, and are a subset of a larger class of problems that include longest path problems, most reliable path problems, and largest capacity path problems. Several good 
sources classify the types of SP algorithms available. Deo and Pang [9] classified different algorithms based on a number of factors with the goal of identifying suitable candidates for parallel implementation and computation; the majority of the material for this section was obtained (unless otherwise noted) from this source.

As stated above, SP algorithms are dependent on a number of problem factors. One of these factors is the characteristics of the network. Some of the network characteristics that must be considered are:

- Are the nodes in the network densely or sparsely connected?

- Are the arcs of the network directed (have one direction) or undirected?

- Are the lengths of the arcs positive values or any real value?

- Are the lengths of the arcs based on uniform (unit) distance, euclidean distance, or a more generalized real valued function?

- Are the lengths of the arcs deterministic (fixed) or probabilistic?

In comparing these characteristics to a telecommunications network, we find the transport network can be generally represented as a sparsely connected, undirected graph with positive arc values based on a deterministic function related to euclidean distance. While the pure euclidean distance can be used, several factors can be combined to determine the cost function for a transport facility such as cost of use, type of equipment, and network delay factors.

The type of solution required is another factor in determining the desired SP algorithm. The solution will be governed by a number of characteristics, including:

- Are there any constraints on the selection of the path (i.e. the path must have a certain number of arcs, or pass through a specific node) or is the solution unconstrained?

- Must the solution provide the shortest path only or define the 2nd, 3rd...K-th shortest paths?

- Will the solution determine the path between two specific nodes, between one node and all other nodes in the network, or the shortest paths between all nodes? 
In transport networks the paths for restoration routing are typically unconstrained, but the other characteristics are not as clearly defined, allowing for a number of possible routing methods. If the transport network has sufficient capacity in its protection channels to accommodate any single facility failure, then a shortest path algorithm would be adequate; what is more likely, however, is that protection channels in the network have different capacities, and that a $\mathrm{K}$-th shortest path algorithm is required. K-th shortest path algorithms were used in the self healing systems discussed previously, where several low capacity alternate routes can be used to reroute the traffic of a single failed high capacity facility.

In most cases the primary requirement for restoration is to provide an alternate route for traffic to the node at the other end of the failed facility, and an algorithm that determines a path between two specific nodes can be implemented. However, since any given network node can have a number of transport facilities, and since we would like to design a routing system that can tolerate the loss of network nodes, an algorithm that can provide a path from a specific node to all other nodes in the network is desirable.

The last major factor that classilies SP algorithms is the technique used to determine the shortest path. Each algorithm can be described by its use of preprocessing and the method used to determine the solution. The majority of these SP techniques involve path finding through general purpose computer simulations, and can be further broken into combinatorial and algebraic techniques.

\subsubsection{Combinatorial techniques}

Combinatorial techniques involve traversing the arcs of a graph and recording information obtained in the process; this method is also called the graph traversal technique. Information recorded while traversing the graph is contained in labels that are associated with the nodes and arcs of the graph; all labels are temporary when the lechnique begins, and all are 
assigned permanent or fixed labels when the process is complete. The method that the labels are assigned and modified further classifies the labelling technique. Most research in the area of labelling methods aim to optimize the basic procedure by a number of methods.

In methods where permanent labels are assigned while the algorithm is being performed are called label setting techniques. This technique was first described by Dijkstra [10], as a method of determining the shortest path between two given nodes $P$ and $Q$ in a graph containing $N$ nodes. The method starts at Node $\mathrm{P}$, and the label for Node $\mathrm{P}$ is made permanent. Starting at $\mathrm{P}$, all the arcs connecting $\mathrm{P}$ to its adjacent nodes are identified and the nodes assigned temporary labels; the label is proportional to the distance from that node to Node P. All of the temporary labels are then examined, and the node with the smallest temporary label is then made permanent. The process is then repeated, with all the nodes connected to the nodes with permanent labels now assigned temporary labels. At each iteration, all nodes connected to permanently labelled nodes are searched and the paths that produce the smallest temporary labels are retained, and the node with the smallest temporary label is then made permanent. In this manner the process continues, adding one node at a time until Node $Q$ is reached; the maximum number of cycles required to perform this technique is $\mathrm{N}-1$. When complete, the labels defined by the technique identify the shortest distance to Node P and the sequence of nodes that define the path, and if allowed to run for $\mathrm{N}-1$ iterations the shortest paths to all nodes are determined. Many implementations of label setting algorithms are available using different data structures in an attempt to optimize the traversing process, resulting in several fast search techniques. The Dijkstra algorithm has also been implemented in a neural network by Fahner [11] as a method of providing planning for real-time robotic motion.

If the labelling technique keeps all labels as temporary labels until all calculations are completed, it is called a label correcting technique. One proposed implementation of a label correcting technique in communications is the use of the Bellman-Ford algorithm as discussed by Ephremides and Verdu [12]. The authors assert that a distributed implementation of the Bellman-Ford 
algorithm is well suited to communications networks, for messages between the nodes are processed to determine the labels for each link. By using this method the nodes in the network can have their information updated asynchronously, no network coordination is required, and the nodes rely only on receiving information from adjacent nodes. If the communications between the nodes is continuous, then each node maintains an accurate knowledge of the shortest path to any node in the network.

\subsubsection{Algebraic techniques}

Algebraic techniques are methods that involve the manipulations of matrices or the use of linear equations to produce a solution. An example of a matrix solution is an all pairs routing method similar to calculating the power of a matrix. Examples of linear equation methods are the organization of the network information as an algebraic system and then solving a set of simultaneous equations. For the purposes of this thesis, these methods are not being considered.

\subsection{Neural networks and telecommunications routing}

As stated earlier, neural networks are being applied to current problems and issues in many different fields, and telecommunications is no exception. A search of neural network and telecommunications literature has identified numerous references where neural networks are being applied to this field; the majority of this research has occurred in the last five years with the increased interest in and availability of neural network research tools. Most of the research performed involves some form of routing decision, but the specific applications, conditions, and routing methods vary. Brown [13] and Melsa et al [14] investigate the use of neural networks to perform routing within digital telephone switches, where the priority is performing the maximum number of input/output connections. Other applications include Hiramatsu [15,16], Chugo at al [17] and Frisiani at al [18] where routing decisions for data networks are made based on the traffic levels within 
transport facilities, network delay times and messages between nodes. Lastly, some of the research made specific reference to performing routing decisions in the transport network under normal or failure conditions; this research will be further discussed in the following paragraphs.

One of the routing methods reviewed was proposed by Rauch and Winarske [19]. In this method an all pairs solution to routing of an $N$ node telecommunications network is produced. For a given network, an NxN capacity matrix defines the link connections between nodes and their capacities, with zero entries inserted where no link is present between nodes. Multiplication of the capacity matrix is used to determine the number of links between two specific nodes in the network. If there are L number of nodes (including originating and destination nodes) comprising the route between two specific nodes, then a set of L Nx1 control vectors are defined to represent the two end nodes and their connections to adjacent nodes in the network. A neural network is then used to converge the vectors to determine the links producing the shortest route, utilizing a gradient approach that minimizes the loss function with respect to the capacity matrix and the control vectors. If a condition such as a link failure occurs, the capacity matrix would be changed and a new route can be determined with a new convergence of the vectors performed by the neural network.

This approach is able to perform routing decisions in a telecommunications network, but a number of shortcomings are apparent. These are:

- Routing is determined from the connectivity of the nodes in the network and the network link capacities, and does not utilize a more general form of network representation that could include factors such as network delays.

- This approach produces a centralized routing method where one neural network performs routing decisions for the entire transport network, rather than a distributed routing method.

- Extensive preprocessing in the form of matrix multiplications are required to prepare control vectors for comvergence.

- As a centralized method, neural network scalability becomes a problem. For the 16 node example used by the authors, 1260 active 
neural elements and 9000 connections are required; for a telecommunications network with 100 nodes and 2000 sourcedestination pairs, the authors estimate that 14,000 active neural elements and 100,000 connections would be required.

As a result of these factors, other methods were researched. The most promising approach found is proposed by Jensen et al [20], where a distributed adaptive routing algorithm is investigated for telecommunications networks. In this approach a neural network is placed within each node of a telecommunications network, and is responsible for routing to adjacent nodes. A multilayer feed forward network resides at each node, with the input layer representing the nodes in the network and the output layer representing the links that connect the specific node to adjacent nodes. By using information on the propagation time of packets arriving from other nodes in the network, a mapping of delays through a given link to any node in the network is developed. The propagation delay values, combined with the adjacent link and the source node information, are then used to train the neural network. To use the trained network, an input neuron representing a specific destination node is asserted, and the output of the neural network will be the adjacent link on the shortest path to the destination node. The neural network in the next node of the telecommunications network in turn will make a routing decision toward the destination node based on similar training to that of the first node. When tested against other routing methods, it was found that after training the neural network routing decisions were of equal performance to static routing tables, and were more flexible in adapting to abnormal network conditions.

This approach overcomes the shortcomings of the previous neural network method. By providing a distributed routing system, decisions can be made locally instead of from a centralized point, resulting in faster response. By defining a neural network structure based on mappings of the network nodes and adjacent links, the network implementation is more scalable. And while network delay was used solely in the approach, more generalized functions can be used to represent the network facilities. One of the drawbacks identified was the need for constant communication between nodes, but in a 
modern transport network this can be accommodated.

\subsection{Attributes of an optimal routing method using neural networks}

From reviewing the background on routing from the viewpoints of telecommunications, routing theory, and existing neural network applications, we can define attributes of a preferred network routing system using neural networks. These are:

- The method must be able to perform routing decisions quickly, given the requirement to adapt io restoration situations. Neural networks offer fast decision times, for once a neural network is trained the speed of the decision is limited to the speed of a simulator in the case of a software implementation or chip delays in the case of a hardware implementation.

- The routing method should be distributed to provide the fast decision times, for centralized methods incur delays from the communications required to report the status of the network, perform the decision, and implement the routing changes. Routing methods discussed by Ephremides and Verdu can be implemented in a distributed manner, and both Grover et al and Jensen et al demonstrated distributed routing with conventional and neural network methods respectively.

- The routing method should be able to provide multiple route alternatives in the case of a network failure. This type of performance is produced by using $K$-th shortest paths routing methods, as implemented in Grover at al for the current telecommunications network.

- The routing method will require information provided by adjacent nodes for performing routing decisions. Ephremides and Verdu, Grover et al and Jensen et al all used messages; while Ephremides and Verdu and Jensen et al used continuous messaging to defined the characteristics of the network, Grover et al used messaging only when a failure occurred to determine restoration routes. The 
transmission and processing of the messages between nodes is the main reason for the length of restoration times simulated by Grover et al; if such a message wave is transmitted in advance of a failure when the network is under normal conditions, the processed signatures could be used to determine the ranking of adjacent links for routing to the nodes in a telecommunications network. This ranking information could then be used as a training set for a neural network.

- The neural networks used should be scalable, for as telecommunications networks change a neural network router must be able to adapt accordingly. of the neural network research reviewed, the method used by Jensen et al appears to scale easily.

The purpose of this thesis is to implement a neural network router with the attributes outlined above. The following paragraphs provide a detailed description of the proposed method.

\subsection{Description of the routing method under study}

The routing method studied in this thesis would perform distributed routing, where each individual node in the network performs routing decisions. These routing decisions are based on the relationship between the originating node where the decision is being made, the desired destination node, and the links that connect the originating node to adjacent nodes in the network. By limiting the routing decision to the originating node's links, the number of alternatives that a neural nework has to consider in performing the routing function is reduced.

As discussed, networks consist of a set of nodes connected to each other by a set of links. For simplicity the sample network used in this thesis will be an undirected connected graph; this means that all links are bidirectional (a link can be used to define a route in either direction) and that at least one route can be defined between any two nodes. A route through a network consists of an originating node, a destination node, and a sequence of intervening links and 
nodes between them that defines a route. One of the intervening links in the route will be a link that connects the originating node to an adjacent node.

As stated above, there will always be at least one route that will connect any two nodes; in most cases there will be multiple routes between them. In order to make a routing decision, some type of criteria is required to allow for comparison of the multiple routes between the originating and destination nodes. One method of providing this criteria is by assigning a weight or value to the links and nodes of the network; a route that is comprised of a sequence of nodes and links is then assigned a weight equal to the sum of the weights of the links and nodes. By relating the weights of the multiple routes to the links that connect the originating node to its adjacent nodes, a pattern is defined that can be used as the criteria for performing routing decisions.

This method can best be shown by example. In Figure 2.9(a), route C-F-J connects originating node $\mathrm{C}$ to destination node $\mathrm{J}$. One of the intervening links in the route will be a link that connects the originating node $C$ to the adjacent node $\mathrm{F}$. In addition to this first route, additional routes from $\mathrm{C}$ to $\mathrm{J}$ can be defined through the other links that connect node $\mathrm{C}$ to its other adjacent nodes. In Figure 2.9(b), three other routes can be defined: route C-E-H-J connects $\mathrm{C}$ to $\mathrm{J}$ through the link that connects node $\mathrm{C}$ to node E; C-D-G-J connects $\mathrm{C}$ to $\mathrm{J}$ through the link that connects node $\mathrm{C}$ to node D; and C-A-B-E-H$\mathrm{J}$ connects $\mathrm{C}$ to $\mathrm{J}$ through the link that connects node $\mathrm{C}$ to node $\mathrm{A}$.

In the example we have identified four separate routes from $\mathrm{C}$ to $\mathrm{J}$, but as yet we cannot make a decision as to which route is the best; some form of criteria is required for us to make a decision. In Figure 2.9(c), weights are added to the links that are in proportion to their geometric distance. By adding the weights of the links for each route together, a weight for each route is determined. The weight for each route is now associated with the link that connects originating node $\mathrm{C}$ to the adjacent node that defines the first part of each route from $\mathrm{C}$ to $\mathrm{J}$. On the basis of comparing the route weights, the route that represents the shortest geometric distance from $\mathrm{C}$ to J is C-F-J, and the link from $\mathrm{C}$ to $\mathrm{F}$ is the first choice for routing. In addition, the ranking of the other 
Figure 2.9(a):

One possible route

from originating node $C$ to destination node $J$ is the route $C-F-J$. One of the links in the route is the link that connects originating node $C$ to node $F$.

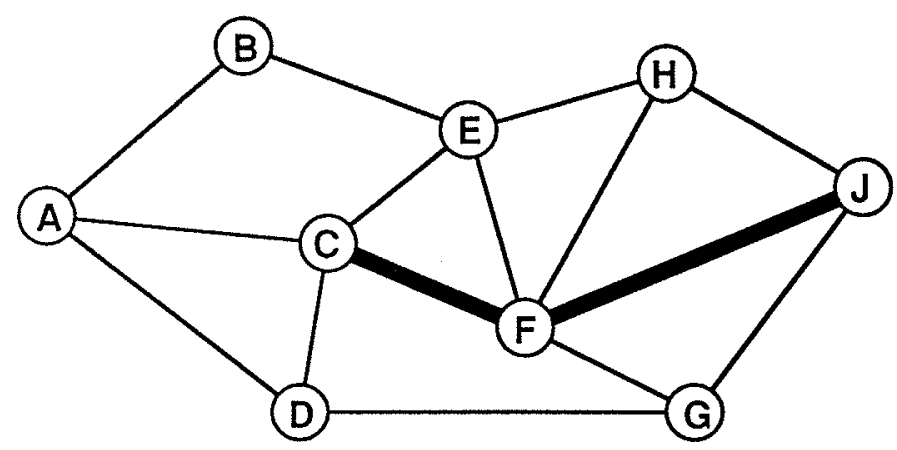

Figure 2.9(b):

Other possible routes

from originating node $C$ to destination node $J$ and their links from originating node $C$ to an adjacent node:

- Route C-E-H-J (link C to E)

- Route C-D-G-J (link C to D)

- Route C-A-B-E-H-J (link C to A)

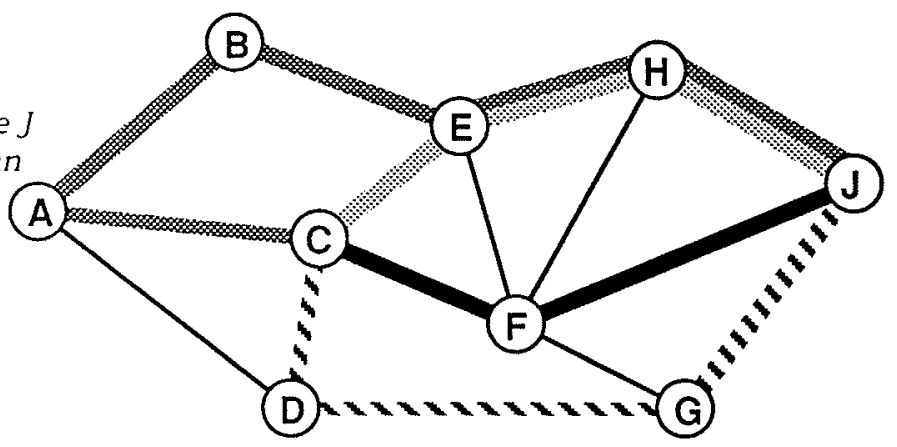

Figure 2.9(c):

Weights are assigned to links. Weighted values for routes are calculated and associaled with links.

\begin{tabular}{ccc} 
Route & link & route value \\
\hline$C-F-J$ & $C$ to $F$ & 0.240 \\
$C-E-H-J$ & Cto $E$ & 0.254 \\
$C-D-G-J$ & C to $D$ & 0.350 \\
$C-A-B-E-H-J$ & C to $A$ & 0.504
\end{tabular}

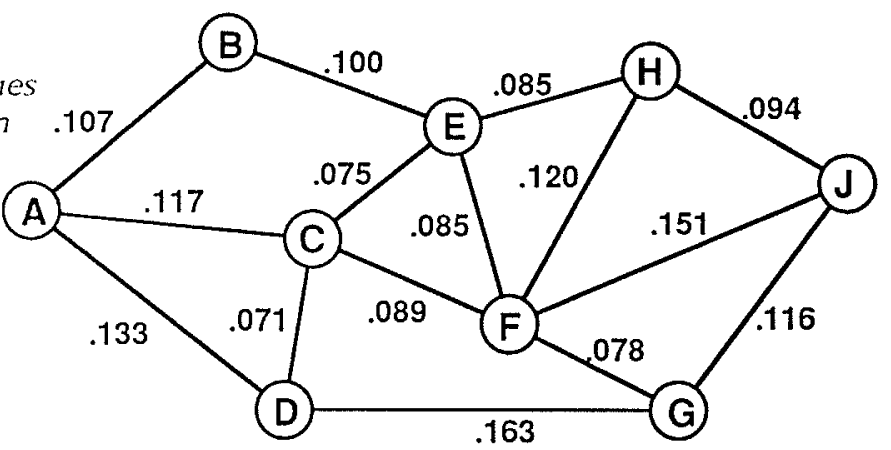

Figure 2.9(d):

Routing process is repeated with node $F$ acting as the originating node.

$\begin{array}{ccc}\text { Route } & \text { link } & \text { roure value } \\ \text { F-C-E-H-J } & \text { Fto } C & 0.343 \\ F-E-H-J & \text { F to } E & 0.264 \\ F-H-J & \text { Fto } H & 0.214 \\ F-J & \text { Fto } J & 0.151 \\ F-G-J & \text { Fto } G & 0.194\end{array}$

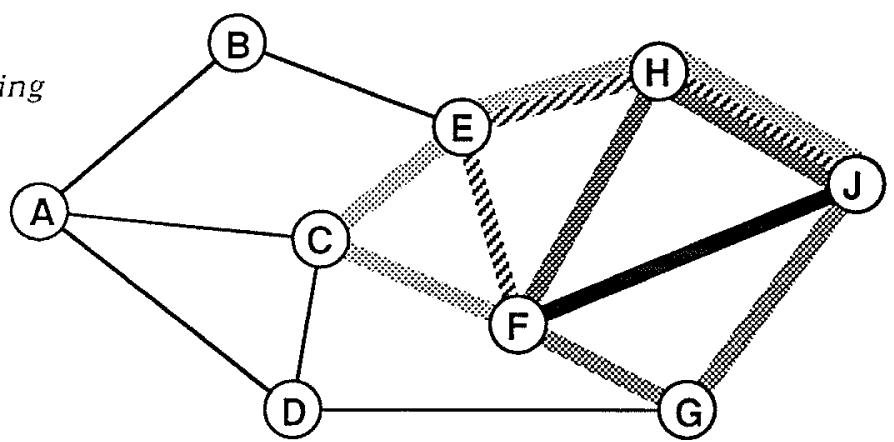


routes has also been determined; routes C-E-H-J, C-D-G-J, and C-A-B-E-H-J are the $2 \mathrm{nd}, 3 \mathrm{rd}$, and 4 th choices for routing, respectively.

By using the above method, the links from a given originating node have been ranked with respect to a weighting factor to the destination node. The routing decision would select the link from node $C$ to node $F$ as the best choice for routing to node $\mathrm{J}$, and a portion of the complete route has been defined. In order to completely determine the route, the same process would be performed with node $\mathrm{F}$ as the originating node trying to determine the best route to node $J$. The process is repeated relating destination node $J$ to the links that connect node $\mathrm{F}$ to its adjacent nodes. In this case node $\mathrm{F}$ is connected by a link to node $\mathrm{J}$, and in determining the weights of the route the direct link would be chosen. The routing example with node $F$ is shown in Figure 2.9(d).

As demonstrated above, the relationship of the originating node, the destination node, and the adjacent links can be used to perform routing. In order to perform the routing decisions properly, however, the routes that are related to the adjacent links must comply with the following rules:

- The routing sequence from the originating node to the destination node through a specific adjacent link must be the best route using that link based on the criteria used for routing. In figure 2.9, route C-A-B-E-H-J and C-A-D-G-J are both routes from originating node $\mathrm{C}$ to destination node $J$, and both use the adjacent link from $C$ to $A$; but route $\mathrm{C}-\mathrm{A}-\mathrm{B}-\mathrm{E}-\mathrm{H}-\mathrm{J}$ has the smallest weighting factor. The best route must be used for each adjacent link in order for the order of the routing choices to be properly determined.

- The routing sequence from the originating node to the destination node through a specific adjacent link must not reuse the originating node. This would lead to incorrect routing decisions and a dependence on another adjacent link connected to the originating node. Please note that in 2.9(d), node $\mathrm{F}$ searched for a route from node $\mathrm{F}$ to node $\mathrm{C}$; this is allowed for node $\mathrm{F}$ has become the originating node performing the routing decision. 
The next section of this thesis will further describe the routing information used in the simulations and experiments. In considering the objectives of this thesis, the collection and creation of the routing information and its relation to the adjacent nodes is not being studied; signature analysis of messages between nodes as discussed previously is one possible method that can perform this function. The objective is to study the ability of a neural network to learn and process this information to perform routing decisions. As will be shown in later sections, the learning of the routing information will allow an individual node to not only determine the link that defines the shortest route to a given destination node, but also determine the best alternate link(s) if the first choice is not available. 


\section{Chapter 3}

\section{Single Network Node Modelling}

In this chapter neural networks will be studied to determine their ability to learn routing information for a single node in a sample network. Several network topologies and training methods will be tested, and modifications to the training set will be performed to determine the effects on learning. From these tests, conclusions will be made on the optimal parameters to perform learning of routing information.

\subsection{Description}

\subsubsection{Description of the sample network}

In order to study the ability of a neural network to perform network routing decisions, a sample network is required to provide the network data needed for the research. The sample network used for this research is shown in Figure 3.1.

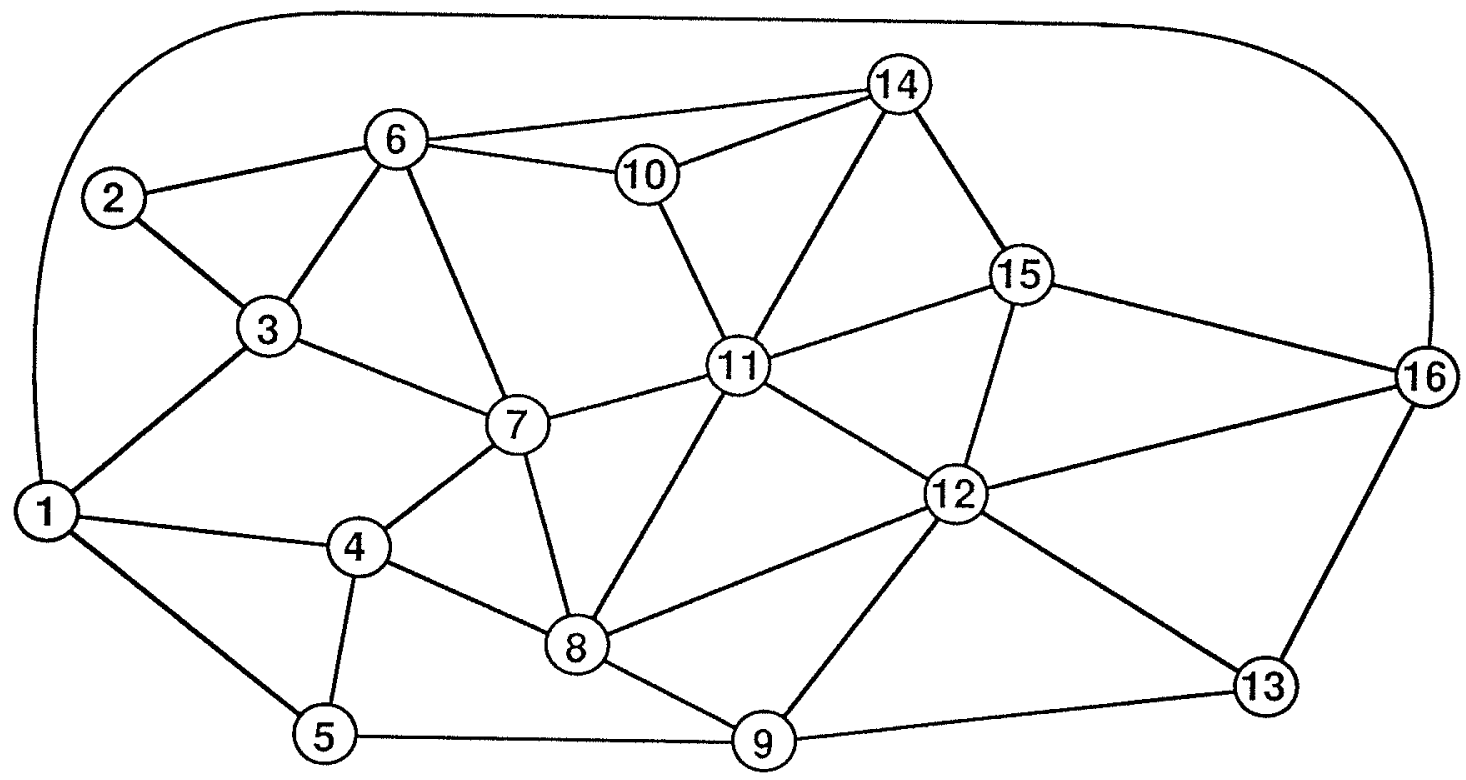

Figure 3.1:

Sample Network 
The sample network is an undirected connected graph illustrative of a simple communications network, consisting of 16 nodes and a set of associated links. The number of links connected to each node (the node's valence) ranges from a 2 to 6 . Table 3.1 lists the valence values, the number of nodes in the sample network of each valence, and the numbers of the nodes.

Table 3.1:

Valence values for the sample network

\begin{tabular}{|c|c|c|}
\hline Valence & \# Nodes & Nodes \\
\hline 2 & 1 & 2 \\
\hline 3 & 3 & $5,10,13$ \\
\hline 4 & 7 & $1,3,4,9,14,15,16$ \\
\hline 5 & 3 & $6,7,8$ \\
\hline 6 & 2 & 11,12 \\
\hline
\end{tabular}

\subsubsection{Description of the neural network}

Figure 3.2 depicts the neural network studied in this thesis and its relationship to the nodes in the sample network. Each neural network is trained to determine routing results for a specific node in the sample network. The neurons in the input layer of the neural network represent the nodes of the sample network. The neurons in the sutput layer of the neural network represent the links of the specilic node in the sample network to its adjacent nodes; in Figure 3.2, links 1.1 to $L 4$ represent the adjacent links connected to node 9. The training set for each neural network consists of routing information relating each node and its adjacent links to the other nodes in the sample network as described in the introduction of this thesis. The task of the neural network is to learn this related routing information for each node. By performing postprocessing on the neural network's outputs, the link that best fits the routing criteria can be determined from the output layer.

In considering the various neural network topologies, activation functions, and learning rules available for use, the following choices were made for the test networks. 


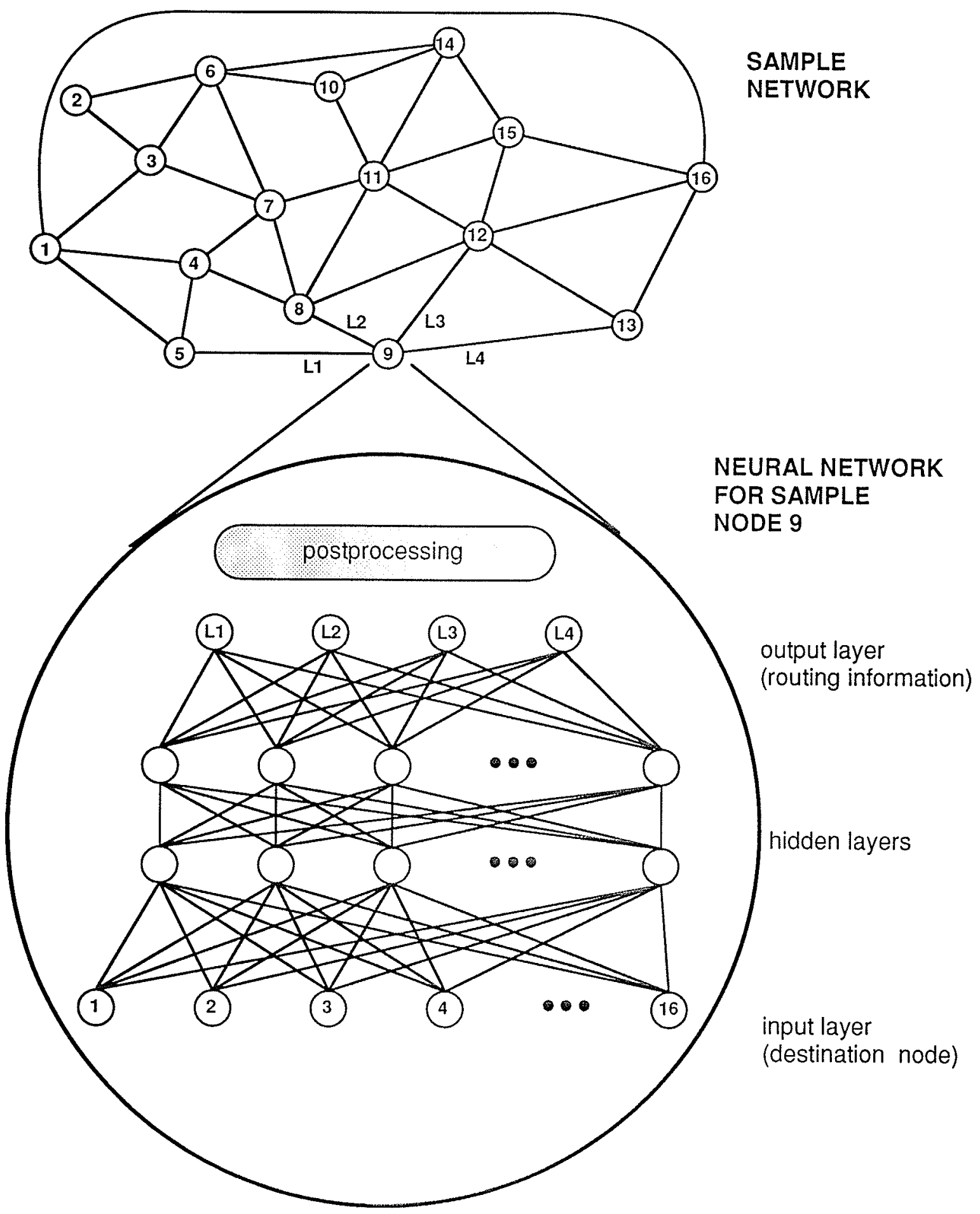

Figure 3.2:

Neural Network and its relation to the Sample Network 


\subsubsection{Learning rule}

The learning rule to be used is Back Propagation. This rule was chosen because the task to be performed is a function of mapping input vectors to output vectors. Back Propagation has been found to be an effective learning technique for mapping and pattern classification problems [21].

\subsubsection{Topology}

The network topology is a lour layer leed forward network consisting of an input, an output, and wo hidden layers. Each layer is fully connected to the following layer. The four layer feed forward network (a three layer perceptron) is commonly used with the Back Propagation learning rule in mapping applications and pattern classification; the output rankings of the neural network can be considered a pattern that must be classified for each input. There are N! possible ranking patterns for a sample network node with $\mathrm{N}$ outputs, and to select the correct paltern oul of a large number of patterns would require a pattern classifier capable of creating complex and arbitrary decision regions. The three layer perceptron is well suited to creating these types of regions [22].

\subsubsection{Activation function and associated variables}

The activation function for the nework is a continuous sigmoid [23], represented by the equation below

$$
a(t+1)=\frac{1}{1+e^{-w(t) / T}}
$$

where

$a(t+1)$ is the activation value of a neuron, $w(t)$ is the weighted sum of the neuron's inputs, including 
any threshold values, and

$\mathrm{T} \quad$ is the network temperature.

For this function, the threshold is set to zero, and the maximum and minimum activation values are set at 0 and 1 respectively; these values are common in back propagation learning. The temperature variable is used to modify the steepness of the sigmoid function, and is usually set high at the start of learning to provide a gradual activation function. As learning progresses the temperature is reduced, resulting in a steeper activation function that forces the network towards the minimum error solution.

\subsubsection{Learning rate and momentum}

The back propagation learning rule determines the error between a neuron's actual output values and the desired values, and modifies the weight of the connection to the previous layer; this process starts at the output layer and is propagated back to through the neural network to the input layer. The formula [24] that determines the change in connection weight is shown below

$$
\Delta w(t)=-\alpha \frac{\partial E}{\partial w}(t)+\sigma \Delta w(t-1)
$$

where

$w(t) \quad$ is the weight of the comnection between two nodes in dillerent layers,

$\Delta w(t) \quad$ is the change in connection weight through learning,

$\mathrm{E} \quad$ is the global error (the squared error between the actual and desired output values),

$\partial E(t) / \partial w$ is the rate of change of the global error with respect to the connection weight,

$\alpha \quad$ is the learning rate (a variable that controls the amount of weight change) and

$\sigma \quad$ is momentum (a variable that speeds learning by using previous weight change information). 
By adjusting the values of the learning rate and momentum variables, the rate of learning can be controlled. For this thesis, values of 0.2 and 0.9 used for the learning rate and momentum respectively; the values were defaults supplied by the neural network simulator, and were left unchanged.

\subsubsection{Determination of the routing information}

As discussed in the introduction, routing criteria can be established by assigning weights or values to the links and nodes of a network, and using the sum of the weights of a sequence on links and nodes to determine the weight of a route. This routing informalion is the basis of the training sets used in this thesis. In telecommunications neworks, a weight related to distance (physical separation of nodes in a communications network) or time (the time to transmit a signal between nodes) could be used for the study. For this thesis a weighting factor related (o) time was chosen, for a time related factor can be used to model both the links and nodes of a communications network.

The links and nodes of a telcommunications network introduce time delays through different mothods. I'ime deliys incurred by a transport facility (transport delays) are a function of the physical distance between two nodes in the network; in this case a signal with a known velocity travels down the facility, with the delay equal to the distance divided by velocity. Examples of links in a telecommunications nework include copper wire, fibre optic cable, and microwave radio. Time delays in a network node are incurred due to the time required to switch a given signal beween links in and out of a node. An example of a equipment that performs this function is a telephone switch or a DCS, which connects different digital signal inputs to outputs by using a combination of time and space switching; time switching involves the switching of a signal between different time slots, and this process introduces delays. Depending of the nature of a signal and the complexity of a telecommunications node, multiple switches may be performed. By using time based weighting, very dissimilar transport and switching activities can be modelled in the same newwrk, since the artivities all relate to a time 
component.

Depending on the geographical size of the telecommunications network being considered, the comparative magnitude of the weighting for the nodes will change with respect to the links. If the values of the node and link weights are similar in size, then the time delays of the links are small, and the network being modelled will have a small geographic area in telecommunications network terms; such a network model would represent a city. If, however, the node weights are small in comparison to the link weights, then the network would cover a large geographic area, more closely modelling a large network between cities. In this thesis the goal is to model large scale networks, so the link weights would be several times larger than the node weights.

For the purposes of this thesis, a value of 0.25 milliseconds was chosen to approximate the maximum time delay for a network node; this is representative of an average delay in current telecommunications networks. The lengths of the links shown in Figure 3.1 were measured and provided in Table 3.2. If the unmodified link lengths were to be used for the weights, the link weights would be between 8 and 60 times larger than the node weights, which would be too large a difference in magnitude for the desired simulations. To reduce the difference in magnitude between link and node weights, the link weights were multiplied by a factor of 0.37 ; as a result the link weights were now between 3 and 20 limes the size of the node weights. The value of 0.37 was produced by using the following formula

$$
\frac{(7600000)(1.46)}{(300)(100000)}=0.37 \quad(\mathrm{msec} / \mathrm{cm})
$$

where

$$
\begin{array}{lll}
7600000 & = & \text { Scaling factor } \\
1.46 & = & \text { index of refraction } \\
300 & = & \text { Speed of } l i g h t(\mathrm{~km} / \mathrm{msec}) \\
100000 & = & \text { number of }(\mathrm{entimeters} \mathrm{per} \mathrm{kilometer}(\mathrm{cm} / \mathrm{km})
\end{array}
$$


Table 3.2

Length values for each link in the sample network

\begin{tabular}{|c|c|c|c|c|c|c|c|c|c|c|c|c|c|c|c|c|}
\hline From/To & 1 & 2 & 3 & 4 & 5 & 6 & 7 & 8 & 9 & 10 & 11 & 12 & 13 & 14 & 15 & 16 \\
\hline 1 & & & 2.9271 & 3.1907 & 3.6200 & & & & & & & & & & & 15.000 \\
\hline 2 & & & 2.0330 & & & 2.9271 & & & & & & & & & & \\
\hline 3 & 2.9271 & 2.0330 & & & & 2.2895 & 2.7127 & & & & & & & & & \\
\hline 4 & 3.1907 & & & & 1.9311 & & 2.0330 & 2.4180 & & & & & & & & \\
\hline 5 & 3.6200 & & & 1.9311 & & & & & 4.4450 & & & & & & & \\
\hline 6 & & 2.9271 & 2.2895 & & & & 3.1269 & & & 2.5598 & & & & 4.9621 & & \\
\hline 7 & & & 2.7127 & 2.0330 & & 3.1269 & & 2.3114 & & & 2.3114 & & & & & \\
\hline 8 & & & & 2.4180 & & & 2.3114 & & 2.1298 & & 3.2690 & 4.1275 & & & & \\
\hline 9 & & & & & 4.4450 & & & 2.1298 & & & & 3.1750 & 5.1196 & & & \\
\hline 10 & & & & & & 2.5598 & & & & & 2.1298 & & & 2.7127 & & \\
\hline 11 & & & & & & & 2.3114 & 3.2690 & & 2.1298 & & 2.5598 & & 3.2690 & 3.0122 & \\
\hline 12 & & & & & & & & 4.1275 & 3.1750 & & 2.5598 & & 3.7026 & & 2.3114 & 4.9289 \\
\hline 13 & & & & & & & & & 5.1196 & & & 3.7026 & & & & 3.5497 \\
\hline 14 & & & & & & 4.9621 & & & & 2.7127 & 3.2690 & & & & 2.2896 & \\
\hline 15 & & & & & & & & & & & 3.0122 & 2.3114 & & 2.2896 & & 4.2360 \\
\hline 16 & 15.000 & & & & & & & & & & & 4.9289 & 3.5497 & & 4.2360 & \\
\hline
\end{tabular}


To use the values in the above formula in an illustrative example, if the sample network was drawn to a 1:7 600000 scale, and the velocity of a signal was the speed of light divided by the given index of refraction, the factor of 0.37 milliseconds/ centimeter is produced. To provide an idea of the scale used, Figure 3.3 shows the sample network superimposed on a geographic area of equivalent scale.

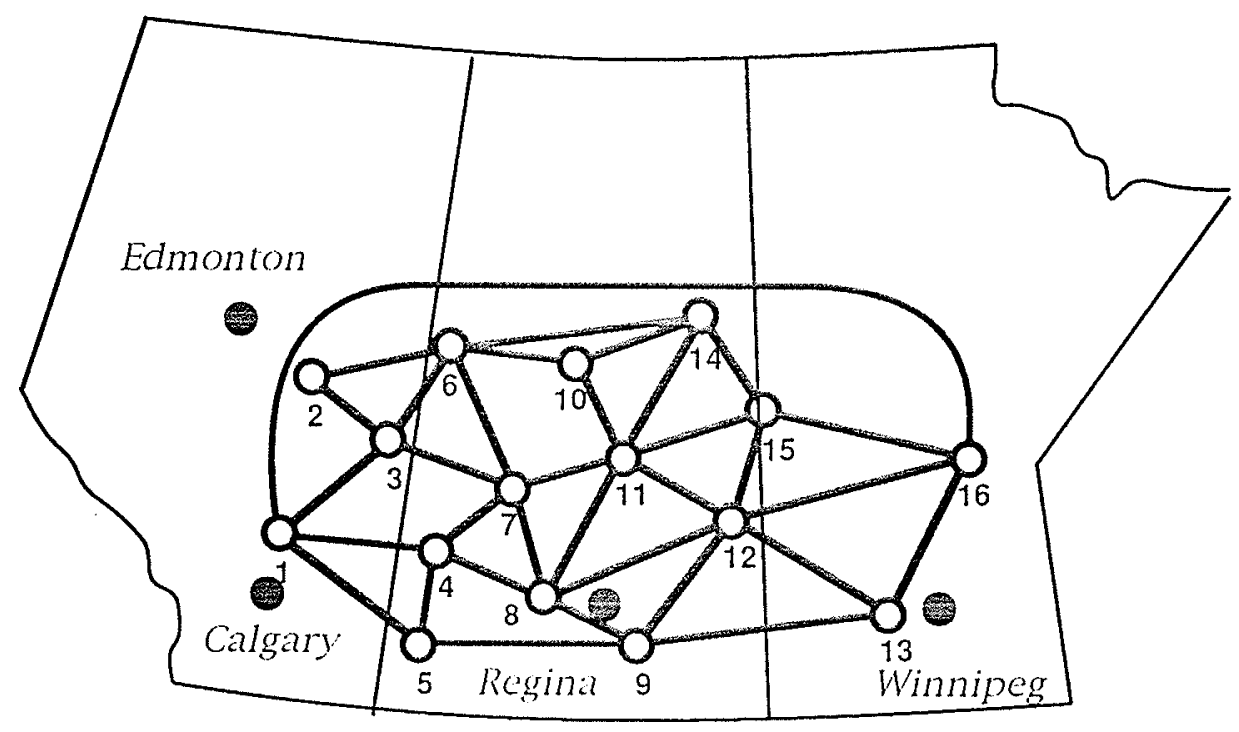

ligure 3.3:

Comparison of sample ncuwork to a gcographic area of equivalent scale.

After performing the described scaling, the new values are provided in Table 3.3(a). When the weights of several sample sequences of nodes and links were summed, it was found that the route weights were too large to be used as output values for training in back propagation neworks; the route weights must be between 0 and 1 for the networks, activation functions, and learning paradigms to be used. By multiplying all of the weights by 0.1 , the weights were reduced to values where the route weights could be used for training purposes. The resulting final weighting values for the nodes and links are provided in 3.3 (b). 
Table 3.3 (a)

Scaled node and link weights

\begin{tabular}{|c|c|c|c|c|c|c|c|c|c|c|c|c|c|c|c|c|}
\hline From/To & 1 & 2 & 3 & 4 & 5 & 6 & 7 & 8 & 9 & 10 & 11 & 12 & 13 & 14 & 15 & 16 \\
\hline 1 & 0.2500 & & 1.0734 & 1.1700 & 1.3275 & & & & & & & & & & & 5.5005 \\
\hline 2 & & 0.2500 & 0.7455 & & & 1.0734 & & & & & & & & & & \\
\hline 3 & 1.0734 & 0.7455 & 0.2500 & & & 0.8396 & 0.9947 & & & & & & & & & \\
\hline 4 & 1.1700 & & & 0.2500 & 0.7081 & & 0.7455 & 0.8867 & & & & & & & & \\
\hline 5 & 1.3275 & & & 0.7081 & 0.2500 & & & & 1.6300 & & & & & & & \\
\hline 6 & & 1.0734 & 0.8396 & & & 0.2500 & 1.1466 & & & 0.9387 & & & & 1.8196 & & \\
\hline 7 & & & 0.9947 & 0.7455 & & 1.1466 & 0.2500 & 0.8476 & & & 0.8476 & & & & & \\
\hline 8 & & & & 0.8867 & & & 0.8476 & 0.2500 & 0.7810 & & 1.1987 & 1.5136 & & & & \\
\hline 9 & & & & & 1.6300 & & & 0.7810 & 0.2500 & & & 1.1643 & 1.8774 & & & \\
\hline 10 & & & & & & 0.9387 & & & & 0.2500 & 0.7810 & & & 0.9947 & & \\
\hline 11 & & & & & & & 0.8476 & 1.1987 & & 0.7810 & 0.2500 & 0.9387 & & 1.1987 & 1.1046 & \\
\hline 12 & & & & & & & & 1.5136 & 1.1643 & & 0.9387 & 0.2500 & 1.3577 & & 0.8476 & 1.8074 \\
\hline 13 & & & & & & & & & 1.8774 & & & 1.3577 & 0.2500 & & & 1.3017 \\
\hline 14 & & & & & & 1.8196 & & & & 0.9947 & 1.1987 & & & 0.2500 & 0.8396 & \\
\hline 15 & & & & & & & & & & & 1.1046 & 0.8476 & & 0.8396 & 0.2500 & 1.5533 \\
\hline 16 & 5.5005 & & & & & & & & & & & 1.8074 & 1.3017 & & 1.5533 & 0.2500 \\
\hline
\end{tabular}

Internal (Node) Weight: $\quad 0.25$ Link Weight Multiplier: $\quad 0.3667$

Table $3.3(b)$

Final network weights (node and link weights mulliplied by 0.1 )

\begin{tabular}{|c|c|c|c|c|c|c|c|c|c|c|c|c|c|c|c|c|}
\hline From/To & 1 & 2 & 3 & 4 & 5 & 6 & 7 & 8 & 9 & 10 & 11 & 12 & 13 & 14 & 15 & 16 \\
\hline 1 & 0.0250 & & 0.1073 & 0.1170 & 0.1327 & & & & & & & & & & & 0.5501 \\
\hline 2 & & 0.0250 & 0.0746 & & & 0.1073 & & & & & & & & & & \\
\hline 3 & 0.1073 & 0.0746 & 0.0250 & & & 0.0840 & 0.0995 & & & & & & & & & \\
\hline 4 & 0.1170 & & & 0.0250 & 0.0708 & & 0.0746 & 0.0887 & & & & & & & & \\
\hline 5 & 0.1327 & & & 0.0708 & 0.0250 & & & & 0.1630 & & & & & & & \\
\hline 6 & & 0.1073 & 0.0840 & & & 0.0250 & 0.1147 & & & 0.0939 & & & & 0.1820 & & \\
\hline 7 & & & 0.0995 & 0.0746 & & 0.1147 & 0.0250 & 0.0848 & & & 0.0848 & & & & & \\
\hline 8 & & & & 0.0887 & & & 0.0848 & 0.0250 & 0.0781 & & 0.1199 & 0.1514 & & & & \\
\hline 9 & & & & & 0.1630 & & & 0.0781 & 0.0250 & & & 0.1164 & 0.1877 & & & \\
\hline 10 & & & & & & 0.0939 & & & & 0.0250 & 0.0781 & & & 0.0995 & & \\
\hline 11 & & & & & & & 0.0848 & 0.1199 & & 0.0781 & 0.0250 & 0.0939 & & 0.1199 & 0.1105 & \\
\hline 12 & & & & & & & & 0.1514 & 0.1164 & & 0.0939 & 0.0250 & 0.1358 & & 0.0848 & 0.1807 \\
\hline 13 & & & & & & & & & 0.1877 & & & 0.1358 & 0.0250 & & & 0.1302 \\
\hline 14 & & & & & & 0.1820 & & & & 0.0995 & 0.1199 & & & 0.0250 & 0.0840 & \\
\hline 15 & & & & & & & & & & & 0.1105 & 0.0848 & & 0.0840 & 0.0250 & 0.1553 \\
\hline 16 & 0.5501 & & & & & & & & & & & 0.1807 & 0.1302 & & 0.1553 & 0.0250 \\
\hline
\end{tabular}


With the weighting values for the nodes and links determined, pattern files containing the routing information for a set of sample nodes were generated. It was decided that a small set of sample nodes would be initially tested to evaluate the performance of the neural networks. The sample nodes chosen were nodes 2, 9 and 11; these nodes have 2, 4, and 6 adjacent links respectively, and by using these nodes for the initial testing the results would provide some indication of the performance of the neural networks for small, medium, and large numbers of output nodes.

The pattern file for Node 2 is shown in Table 3.4. Each pattern file consists of a set of input/output vector pairs. The input vector is a binary vector that asserts a single input neuron representing a specific destination node. The output vector is a real value vector representing the routing information for that particular destination node derived from the routing criteria discussed. In each pattern file, one of the 16 inpul neurons represents the particular node in the sample network that is being trained; as a result, the output vector for that node would contain zero values. In Table 3.4, these values are associated with input neuron 12 . In the 2, 4, and 6 output node training sets, the corresponding input neurons with zero output vector values are i2, i9, and i1 1 respectively. When the actual training was performed, this zero output value vector pair was excluded, and the training set contained 15 input/output vector pairs instead of 16.

All of the pattern files used in the initial lesting can be found in Appendix A.

\subsubsection{Description of the simulation package}

With the basic neural network lopology, activation function, learning rule and initial training sets defined, the training of the neural networks could be simulated. The network simulations were performed on an Apple Macintosh IIfx with 4MB RAM and an 80) MBB hard drive. The application used to perform the simulations was Mackrain 2.0 and 3.0, distributed by Neurix of Massachusetts. MacBrain is an neural network simulation package capable of 
Table 3.4:

Pattern File for Node 2 of Sample Network

\begin{tabular}{|c|c|c|c|c|c|c|c|c|c|c|c|c|c|c|c|c|c|c|}
\hline \multirow{2}{*}{$\begin{array}{l}\text { Destination } \\
\text { Node }\end{array}$} & \multicolumn{16}{|c|}{ Input Vector } & \multicolumn{2}{|c|}{ Output Vector } \\
\hline & $i 1$ & $i 2$ & i3 & i4 & 15 & i6 & $i 7$ & 18 & i9 & 110 & $i 11$ & 112 & $i 13$ & $i \uparrow 4$ & $i 15$ & $i 16$ & 01 & 02 \\
\hline 1 & 1 & 0 & 0 & 0 & 0 & 0 & 0 & 0 & 0 & 0 & 0 & 0 & 0 & 0 & 0 & 0 & 0.2569 & 0.3986 \\
\hline 2 & 0 & 1 & 0 & 0 & 0 & 0 & 0 & 0 & 0 & 0 & 0 & 0 & 0 & 0 & 0 & 0 & 0.0000 & 0.0000 \\
\hline 3 & 0 & 0 & 1 & 0 & 0 & 0 & 0 & 0 & 0 & 0 & 0 & 0 & 0 & 0 & 0 & 0 & 0.1245 & 0.2663 \\
\hline 4 & 0 & 0 & 0 & 1 & 0 & 0 & 0 & 0 & 0 & 0 & 0 & 0 & 0 & 0 & 0 & 0 & 0.3486 & 0.3966 \\
\hline 5 & 0 & 0 & 0 & 0 & 1 & 0 & 0 & 0 & 0 & 0 & 0 & 0 & 0 & 0 & 0 & 0 & 0.4146 & 0.4924 \\
\hline 6 & 0 & 0 & 0 & 0 & 0 & 1 & 0 & 0 & 0 & 0 & 0 & 0 & 0 & 0 & 0 & 0 & 0.2335 & 0.1573 \\
\hline 7 & 0 & 0 & 0 & 0 & 0 & 0 & 1 & 0 & 0 & 0 & 0 & 0 & 0 & 0 & 0 & 0 & 0.249 & 0.297 \\
\hline 8 & 0 & 0 & 0 & 0 & 0 & 0 & 0 & 1 & 0 & 0 & 0 & 0 & 0 & 0 & 0 & 0 & 0.3588 & 0.4068 \\
\hline 9 & 0 & 0 & 0 & 0 & 0 & 0 & 0 & 0 & 1 & 0 & 0 & 0 & 0 & 0 & 0 & 0 & 0.4618 & 0.5097 \\
\hline 10 & 0 & 0 & 0 & 0 & 0 & 0 & 0 & 0 & 0 & 1 & 0 & 0 & 0 & 0 & 0 & 0 & 0.3524 & 0.2762 \\
\hline 11 & 0 & 0 & 0 & 0 & 0 & 0 & 0 & 0 & 0 & 0 & 1 & 0 & 0 & 0 & 0 & 0 & 0.3588 & 0.3793 \\
\hline 12 & 0 & 0 & 0 & 0 & 0 & 0 & 0 & 0 & 0 & 0 & 0 & 1 & 0 & 0 & 0 & 0 & 0.4776 & 0.4981 \\
\hline 13 & 0 & 0 & 0 & 0 & 0 & 0 & 0 & 0 & 0 & 0 & 0 & 0 & 1 & 0 & 0 & 0 & 0.6385 & 0.659 \\
\hline 14 & 0 & 0 & 0 & 0 & 0 & 0 & 0 & 0 & 0 & 0 & 0 & 0 & 0 & 1 & 0 & 0 & 0.4405 & 0.3643 \\
\hline 15 & 0 & 0 & 0 & 0 & 0 & 0 & 0 & 0 & 0 & 0 & 0 & 0 & 0 & 0 & 1 & 0 & 0.4942 & 0.4733 \\
\hline 16 & 0 & 0 & 0 & 0) & 0 & 0 & 0 & 0 & 0 & 0 & 0 & 0 & 0 & 0 & 0 & 1 & 0.6745 & 0.6536 \\
\hline
\end{tabular}




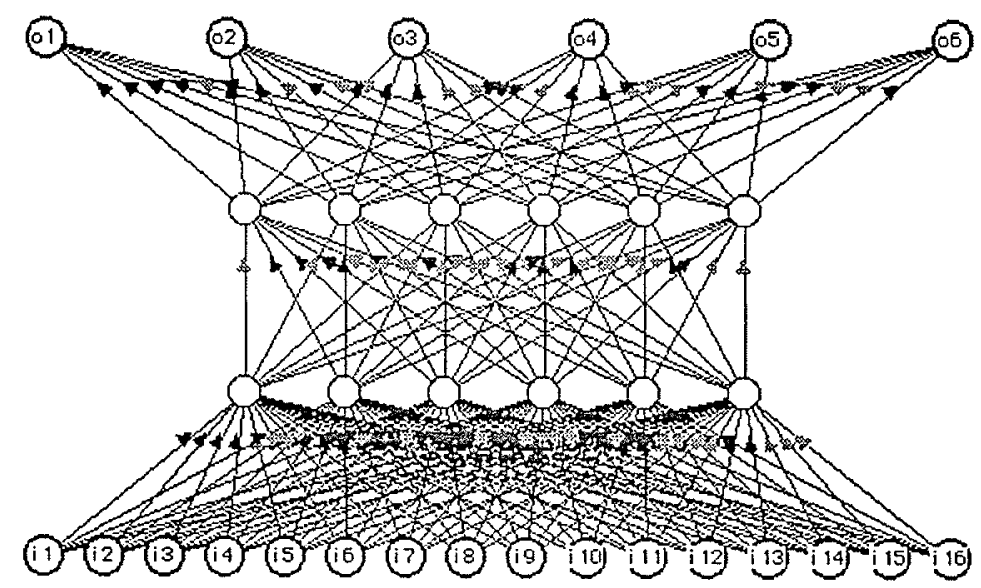

Figure 3.4:

Neural Network with 16 input netrons, 6 output neurons, and 2 hidden layers of 6 neurons each

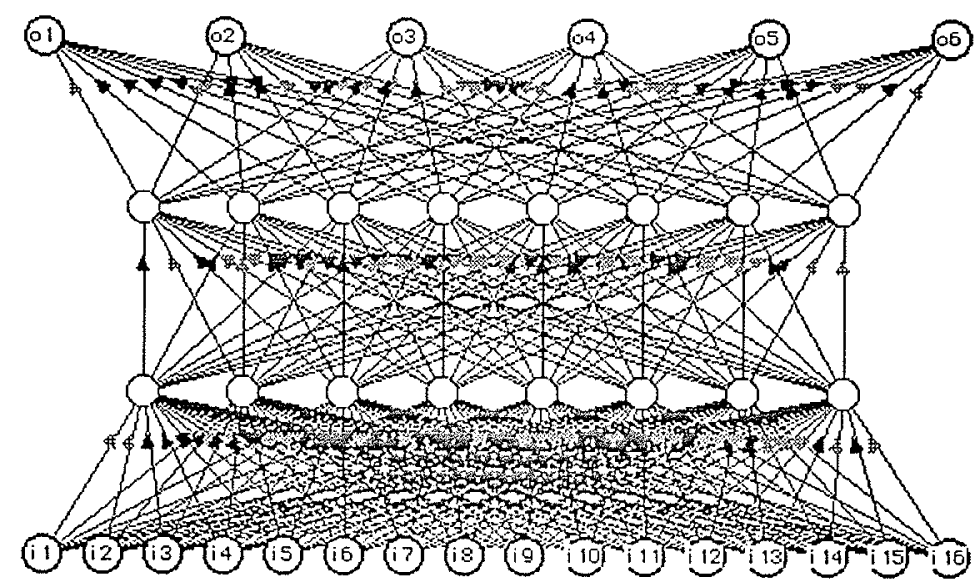

Figure 3.5:

Neural Network with 16 input neurons, 6 output neurons, and 2 hidden layers of 8 neurons each

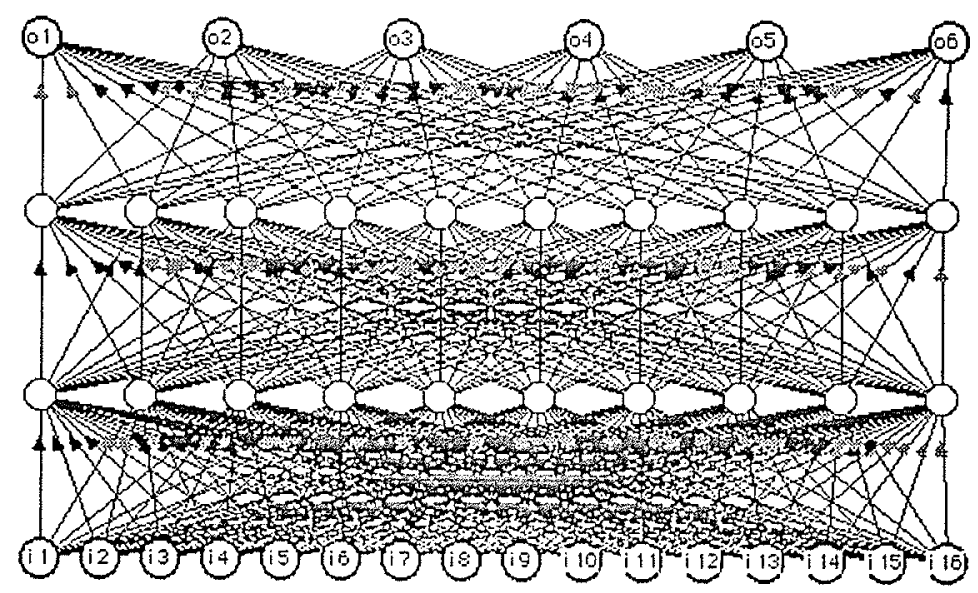

Figure 3.6:

Neural Network with 16 input neurons, 6 output neurons, and 2 hidden layers of 10 neurons each 
simulating different neural network topologies, activation functions, and learning rules.

The first objective of the simulations was to determine a suitable number of neurons for the neural nework's wo hidden layers. For the initial testing three combinations of hidden layers were used; both hidden layers contained equal numbers of neuroms, with each layer having 6, 8, or 10 neurons. Figures 3.4, 3.5, and 3.6 show the topologies of a 6 output neuron, two hidden layer network with 6,8 , and 10 neurons per hidden layer respectively. These networks would become the foundation for all testing; to create a neural network for any of the sample network nodes, output neurons are removed until the desired number is allatined.

\subsection{Training the neural networks}

\subsubsection{The V1 Macro}

The three network copologies were lrained using the three training sets generated for nodes 2, 9, and 11, resulting in 9 (rained neural networks; Table 3.5 lists the nine nework opologics.

Table 3.5:

Netral network topologies used in the initial testing

\begin{tabular}{|c|c|c|}
\hline $\begin{array}{l}\text { Network } \\
\text { Identifier }\end{array}$ & $\begin{array}{l}\text { Description of macro used, number of neurons } \\
\text { per hidden layer, and number of output neurons }\end{array}$ & $\begin{array}{l}\text { Training } \\
\text { set used }\end{array}$ \\
\hline $\mathrm{V} 1.2 .06 .2$ & V1 Macro, 6 neurons/hidden layer, 2 output neurons & Node 2 \\
\hline $\mathrm{V} 1.2 .06 .4$ & V1 Macro, 6 neurons/hidden layer, 4 output neurons & Node 9 \\
\hline $\mathrm{V} 1.2 .06 .6$ & V1 Macro, 6 neurons/hidden layer, 6 output neurons & Node 11 \\
\hline $\mathrm{V} 1.2 .08 .2$ & V1 Macro, 8 neurons/hidden layer, 2 output neurons & Node 2 \\
\hline $\mathrm{V} 1.2 .08 .4$ & V1 Macro, 8 neurons/hidden layer, 4 output neurons & Node 9 \\
\hline $\mathrm{V} 1.2 .08 .6$ & V1 Macro, 8 neurons/hidden layer, 6 output neurons & Node 11 \\
\hline $\mathrm{V} 1.2 .10 .2$ & V1 Macro, 10 neurons/hidden layer, 2 output neurons & Node 2 \\
\hline V1.2.10.4 & V1 Macro, 10 neurons/hidden layer, 4 output neurons & Node 9 \\
\hline $\mathrm{V} 1.2 .10 .6$ & V1 Macro, 10 neurons/hidden layer, 6 output neurons & Node 11 \\
\hline
\end{tabular}


In MacBrain the training of the neural networks is performed by opening a network file containing the neural network and the parameters for its neurons and connections, pasting a pattern file with the training set on to the network, and executing a icon-based flowchart macro that contains the training algorithm. The first algorithm used in testing was called the V1 Macro, with the flowchart for the macro shown in Figure 3.7; the actual V1 Macro graphic can be found in Appendix $A$.

The V1 Macro performs the following functions:

- Prompts the user for the number of training cycles to be executed (NUMCYCLES) and the initial temperature (TEMPERATURE) for the activation function.

- Executes the learning function identified by the Learn node.

- Performs a loop in the program (using the variables INCREMENT and TESTPER) that calculates the mean squared error (NSE), assigns it to the variable MSERROR, and writes it $(0)$ a lext window every 5 learning cycles. In the same loop it reduces the temperature, annealing the network every 5 learning cycles.

- Performs various display housckecping functions.

- Alerts the user when the simulation is complete.

The initial settings for the training were as follows:

- Number of training cycles (NUACYC.ILS):

- Initial temperature (TLAMIRATURE):

- Learning rate:

- Momentum:

0.9

- Method of updating:

Updating by layer

- Learning rulc:

Back Propagation

Balch with Momentum

\subsubsection{Initial analysis of the V1 Macro trained networks}

After the networks were trained the mean squared error results were studied. The MSE graph for nework V1.2.06.4, shown in Figure 3.8, is typical of the 


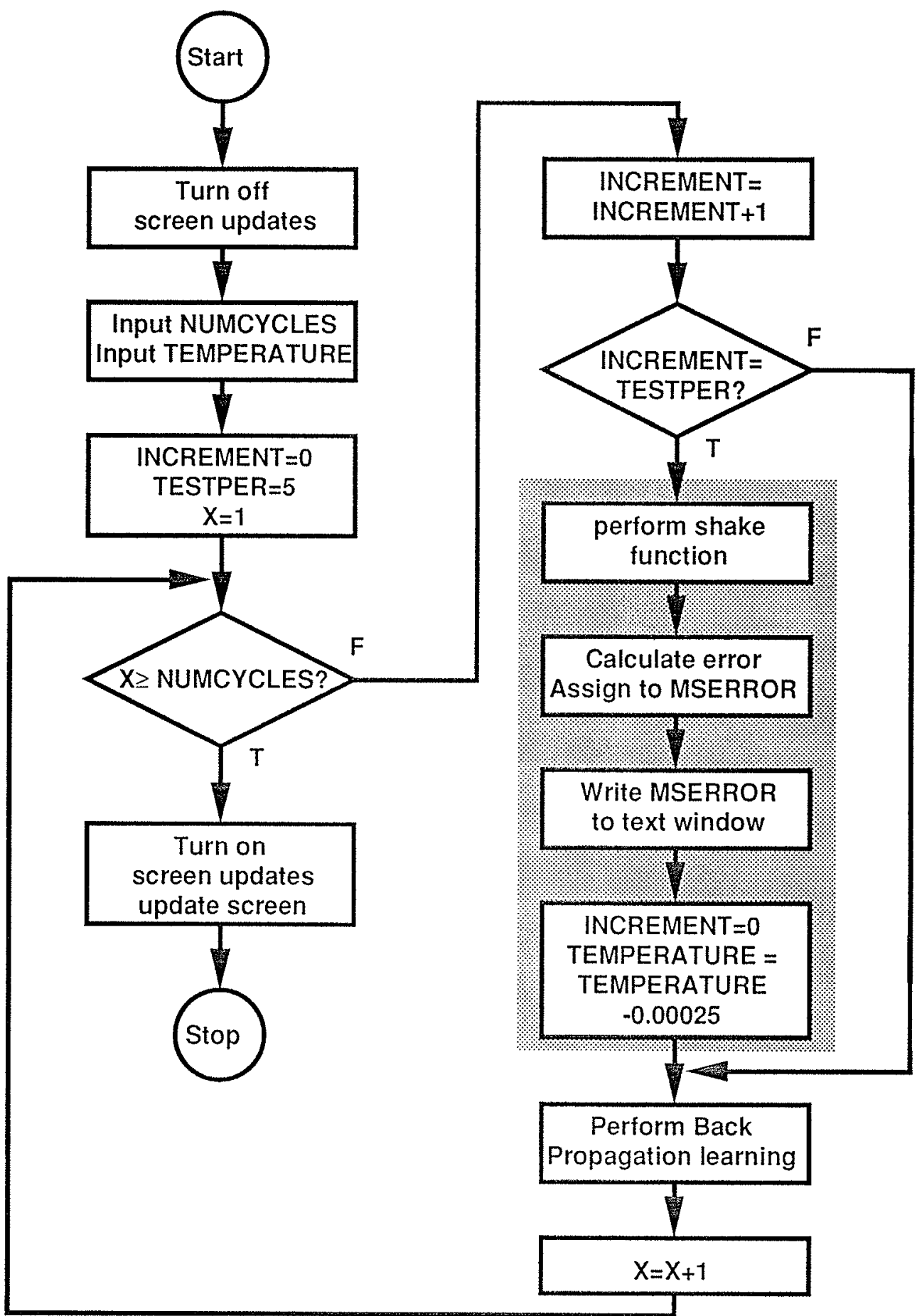

Mean Squared Error calculation and annealing section 


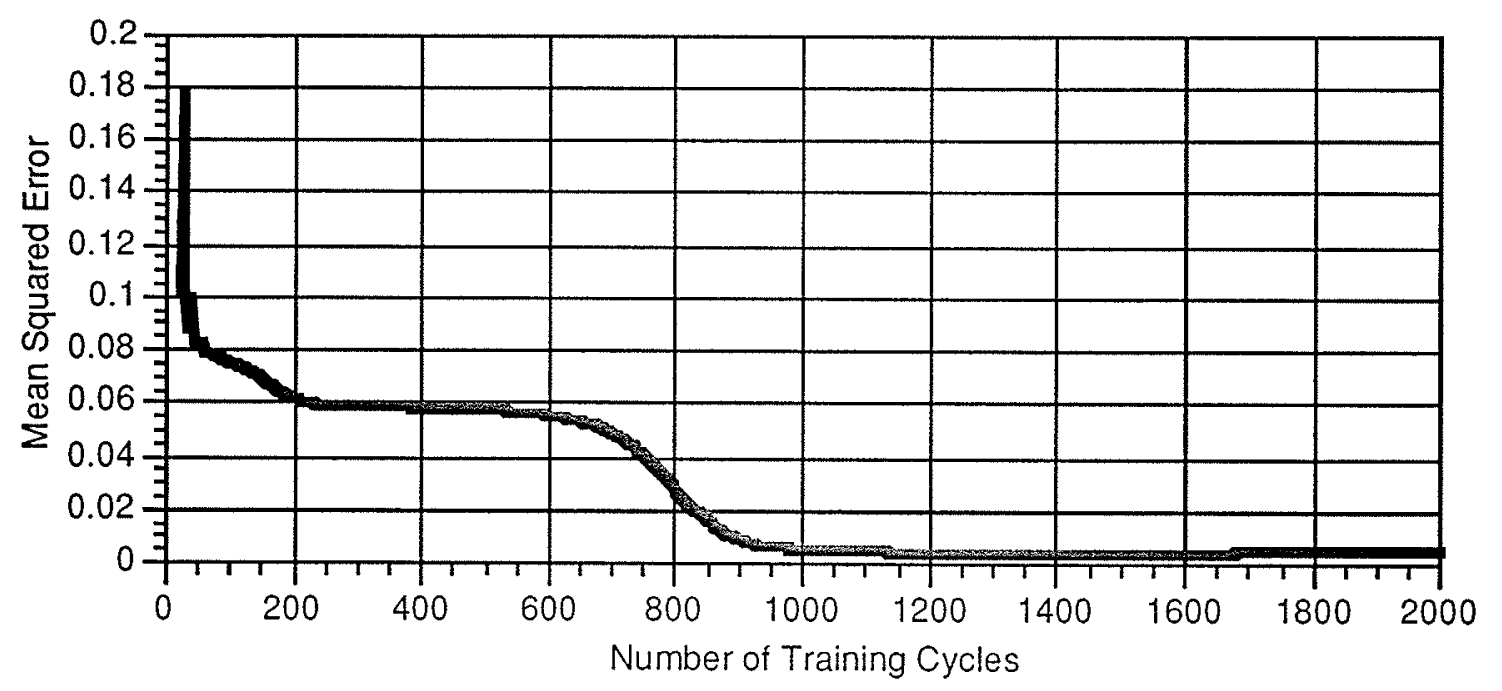

Figure 3.8:

MSE Values as a function of number of training cycles for network V1.2.06.4

mean squared error results for the nine networks trained; for each network the MSE started as a large value and then decreased monotonically after some initial fluctuations. The smooth decrease of the MSE value function is attributed to the fact that the crror was calculated every five training cycles, thus resulting in some degree of smoothing of the function due to sampling. After the initial error fluctuations the function decrease was gradual, followed by a more rapid decrease in the characteristic before once again levelling off; the majority of the learning in the network was probably performed during the rapid decrease in the characteristic. A revicw of the MSE graphs for all nine networks shows that the number of cycles required to reach this point in the characteristic was dependent on the number of output neurons and not the number of hidden neurons per layer. Figures 3.9. 3.10, and 3.11 show the MSE characteristic as a function of 2,4 , and 6 output nodes, respectively. Figures $3.12,3.13$, and 3.14 show the MSE characteristic as a function of 6,8 , and 10 neurons per hidden layer respectively.

One other important feature of each MSE characteristic is that eight of the nine networks reached a minimum MSE: value during the training, and then the MSE values began to increase; this feature is most evident in the V1.2.06.2, which reached the minimum MSE after 685 training cycles. In V1.2.06.6, the 


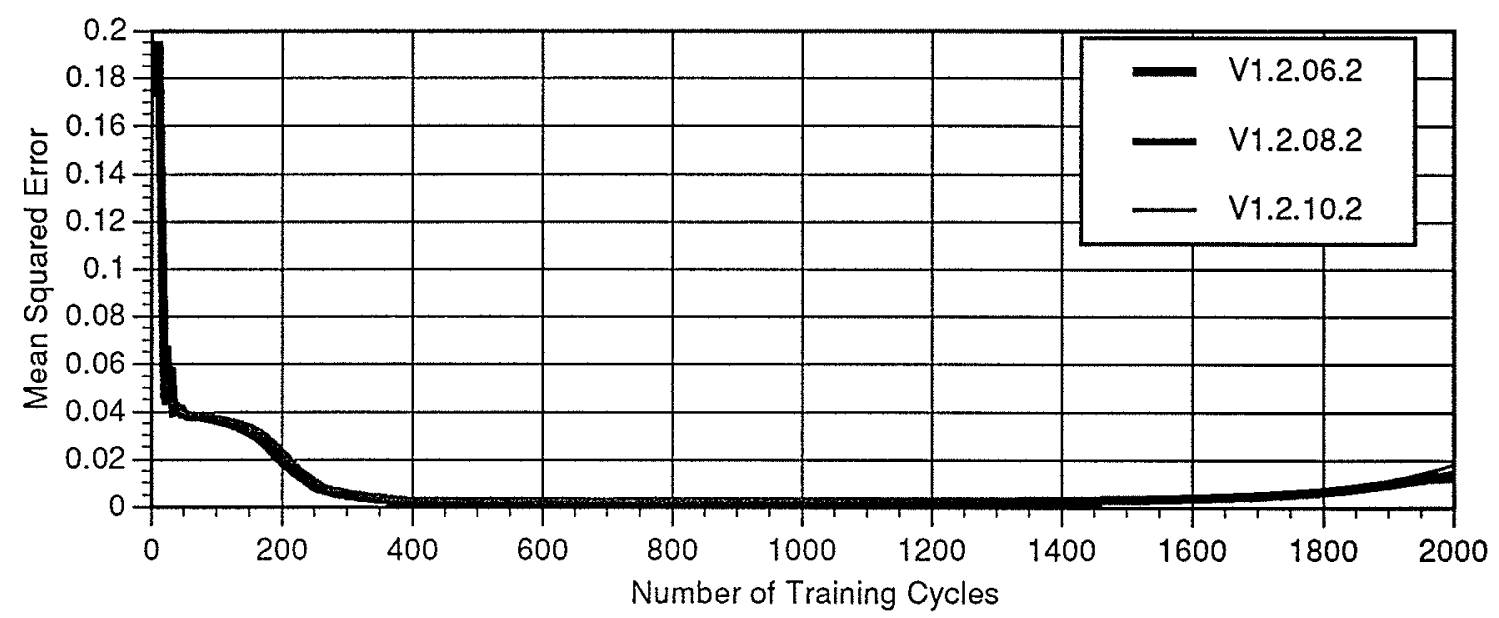

Figure 3.9:

MSE values for networks with two output neurons using the VI Macro.

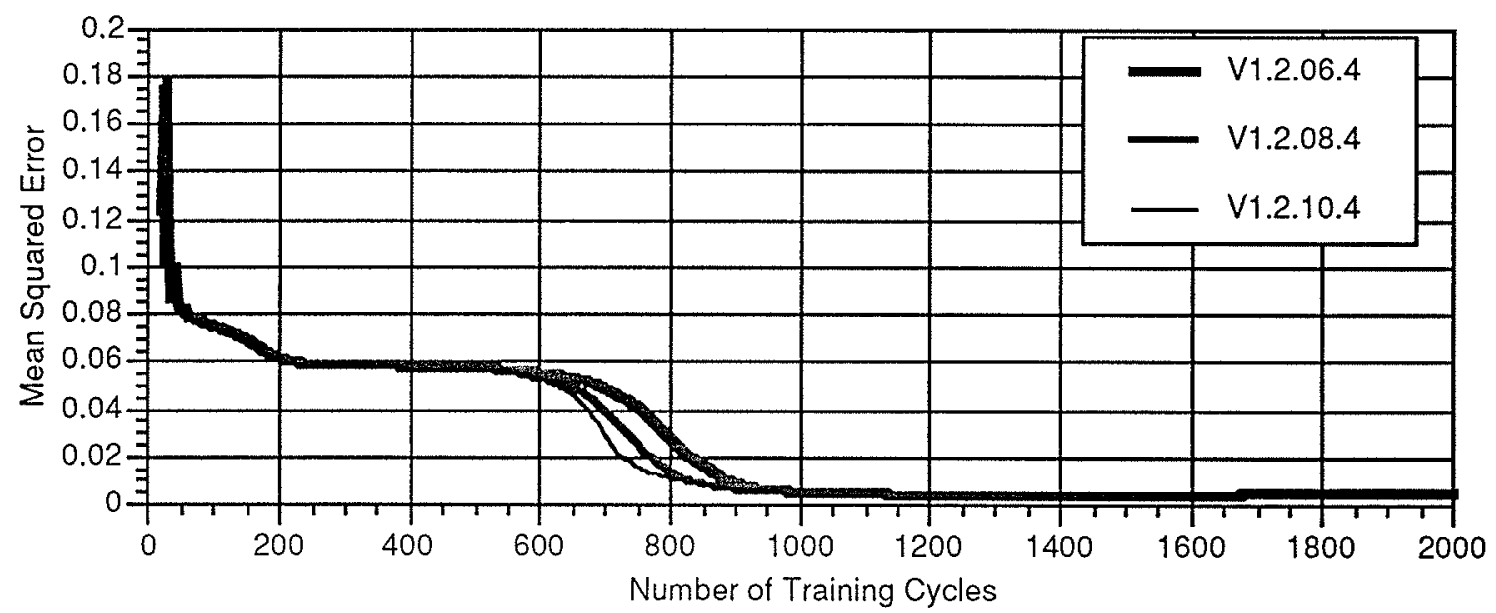

Figure 3.10:

MSE values for networks with four output neurons using the V1 Macro.

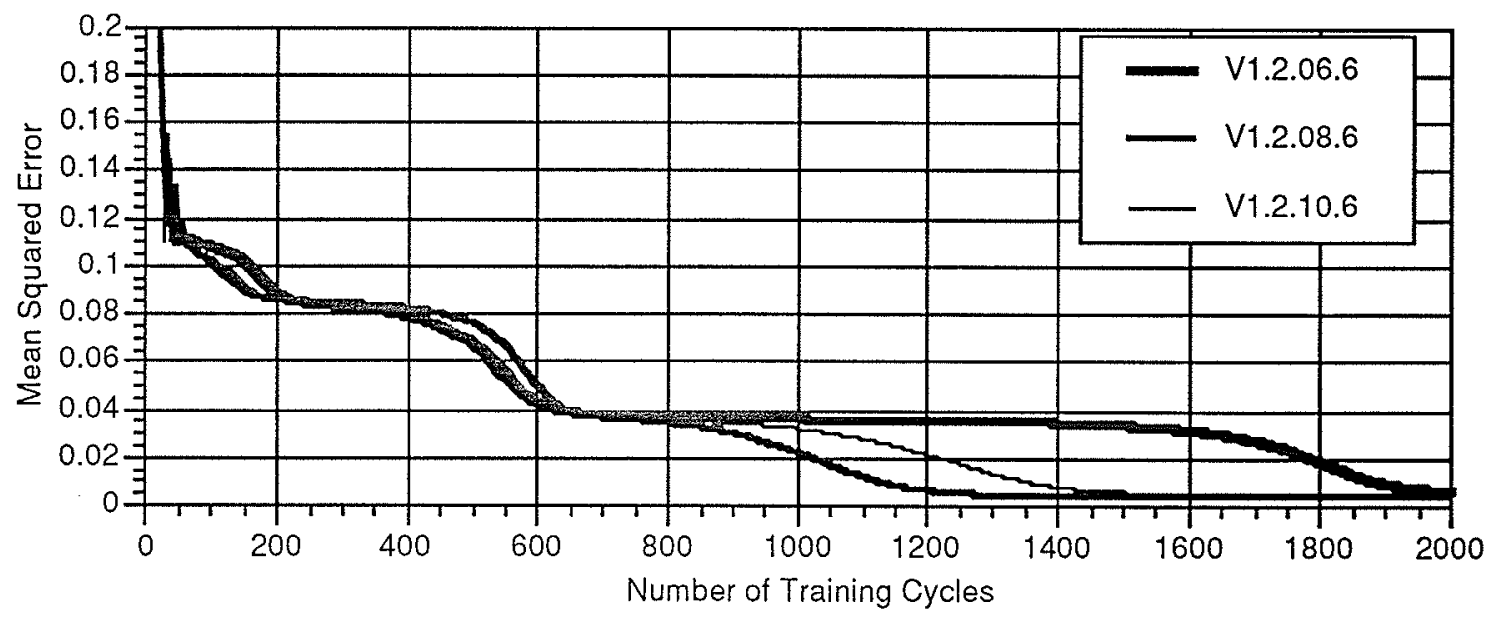

Figure 3.11:

MSE values for networks with six output neurons using the V1 Macro. 


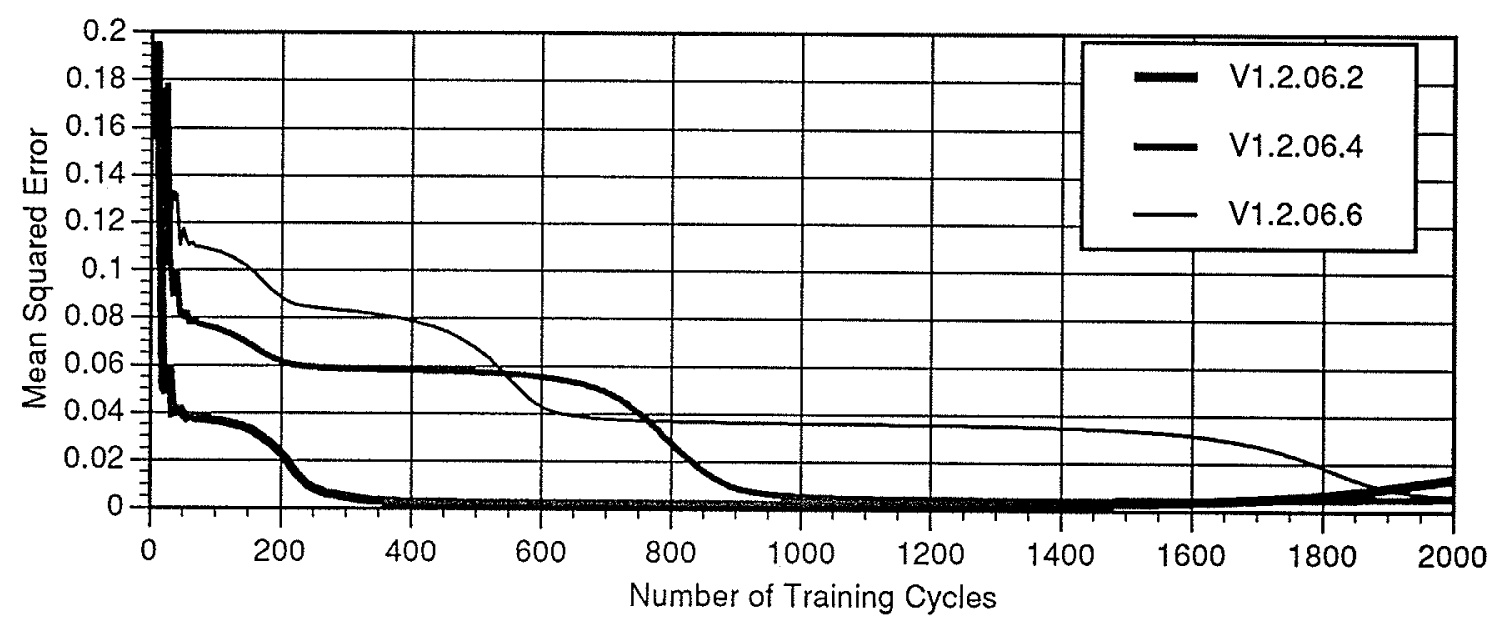

Figure 3.12:

MSE Values for networks with six neurons per hidden layer using the V1 Macro.

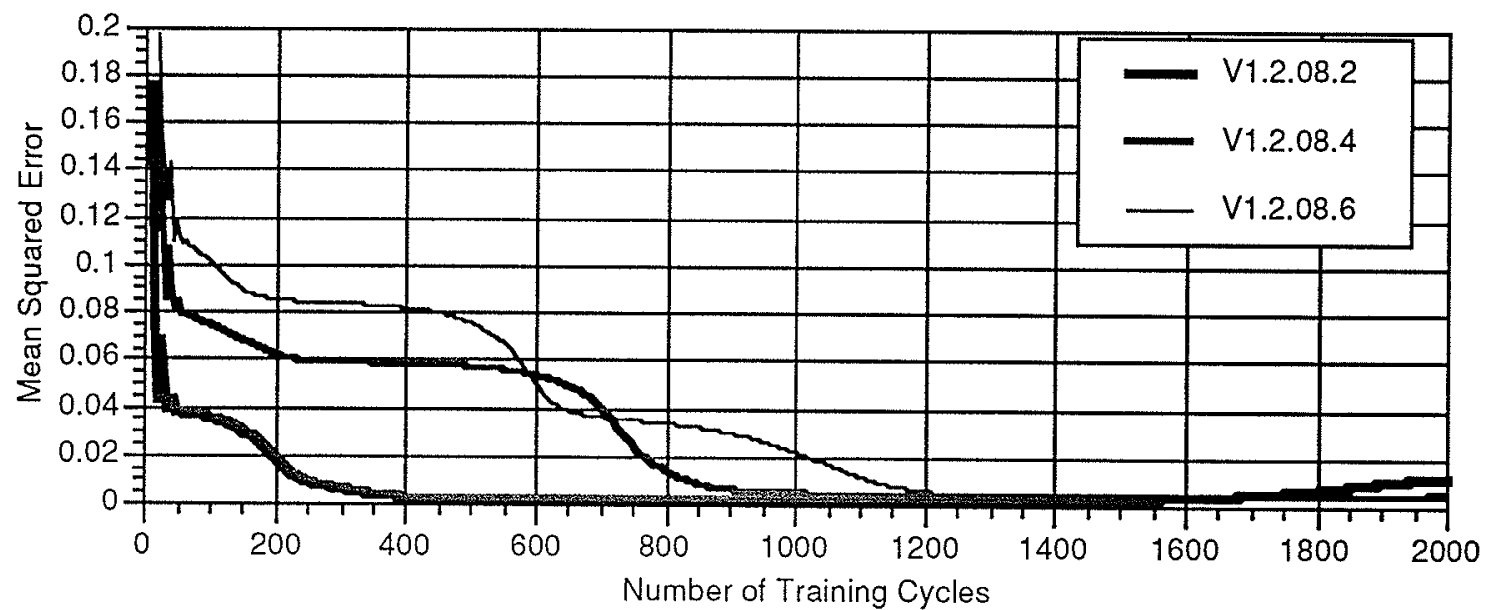

Figure 3.13:

MSE Values for networks with eight neurons per hidden layer using the V1 Macro.

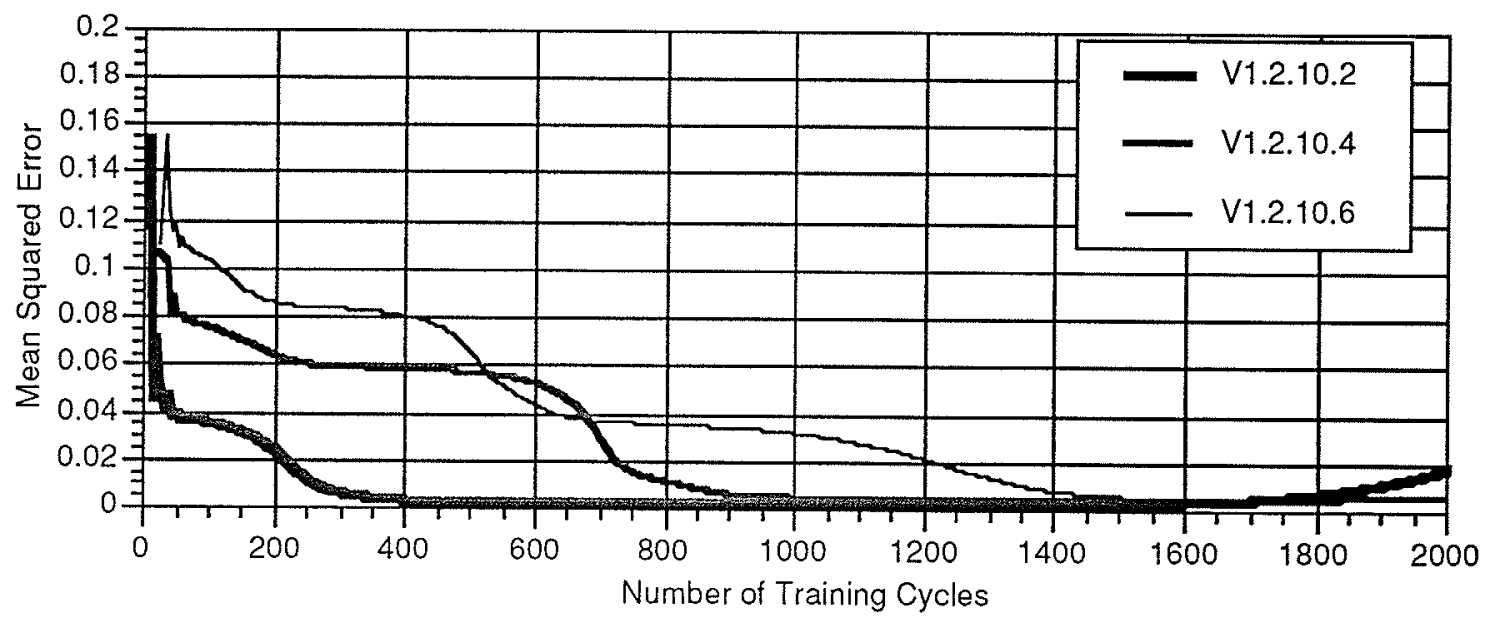

Figure 3.14:

MSE Values for networks with ten neurons per hidden layer using the V1 Macro. 
network did not reach a minimum value within the 2000 cycles; a later training simulation found that this network reached the minimum MSE at 2265 training cycles. Table 3.6 provides a complete listing of each network's minimum MSE and the number of training cycles required to reach the minimum.

Table 3.6:

V.1 Macro minimum MSE results and number of training cycles

\begin{tabular}{|c|c|c|}
\hline Network & Minimum MSE & \# Training cycles \\
\hline $\mathrm{V} 1.2 .06 .2$ & $2.26890 \mathrm{E}-3$ & 685 \\
\hline $\mathrm{V} 1.2 .06 .4$ & $4.41559 \mathrm{E}-3$ & 1375 \\
\hline $\mathrm{V} 1.2 .06 .6$ & $6.57322 \mathrm{E}-3$ & $2000(2265)$ \\
\hline $\mathrm{V} 1.2 .08 .2$ & $2.25664 \mathrm{E}-3$ & 795 \\
\hline $\mathrm{V} 1.2 .08 .4$ & $4.10530 \mathrm{E}-3$ & 1480 \\
\hline $\mathrm{V} 1.2 .08 .6$ & $3.41948 \mathrm{E}-3$ & 1540 \\
\hline $\mathrm{V} 1.2 .10 .2$ & $2.26328 \mathrm{E}-3$ & 875 \\
\hline $\mathrm{V} 1.2 .10 .4$ & $4.26277 \mathrm{E}-3$ & 1300 \\
\hline $\mathrm{V} 1.2 .10 .6$ & $4.83774 \mathrm{E}-3$ & 1750 \\
\hline
\end{tabular}

In considering the cause of the increasing MSE, it was theorized that the V1 Macro's annealing process had reached a point where the decrease in network temperature was no longer assisting the network to reach its minimum energy, and that the steepening activation function was the cause of the increasing error.

To test this theory, a special macro was created that would anneal the network until the minimum MSE was detected, then hold the temperature at a fixed value and let the training continue. An untrained copy of the network 2.06.4 was selected, and the new training macro was executed for 2000 training cycles. The result was thal al the point of minimum MSE a small jump in the MSE value was found as the lomperalure was being fixed, then the MSE characteristic continued 10 decrease as before. Figure 3.15 compares the portion of the V1.2.06.4 characteristic near the minimum MSE point to the new macro's results.

With this result found, it was decided that the next part of the research would compare the output results of the Vl Macro trained networks with two new sets 
of trained networks; the first new set of networks would use the new macro that stops the annealing at the point of minimum MSE and continues learning, and a second set of networks would be trained to the point of minimum MSE. The training for these two sets of networks will now be described.

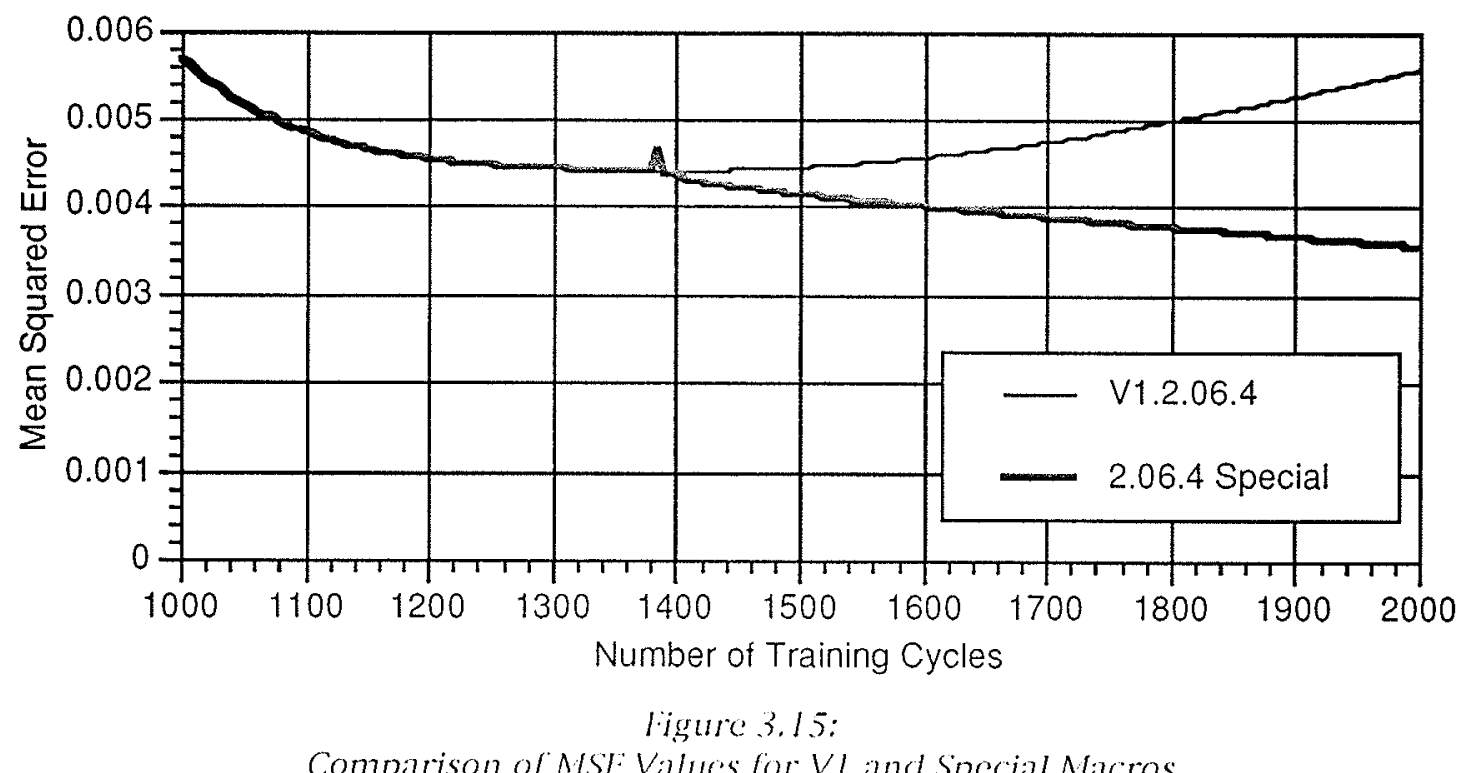

\subsubsection{The V2 and V3 Macros}

The special macro described earlier is called the V2 Macro; the flowchart for the macro shown in ligure 3.16, and the actual V2 Macro graphic can be found in Appendix A.

The V2 Macro is an extension of the VI Macro. This new macro performs the following functions:

- Prompts the user for the number of training cycles to be executed (NUMCYCLES) and the initial temperature (TEMPERATURE) for the activation function.

- Executes the learning function identified by the learn node.

- Performs a loop in the program (using the variables INCREMENT and TESTPER) that calculates the mean squared crror, assigns it to the variable MSERROR, and writes it to a text window every 5 learning cycles. In the same loop the lesting for the minimum MSE is 


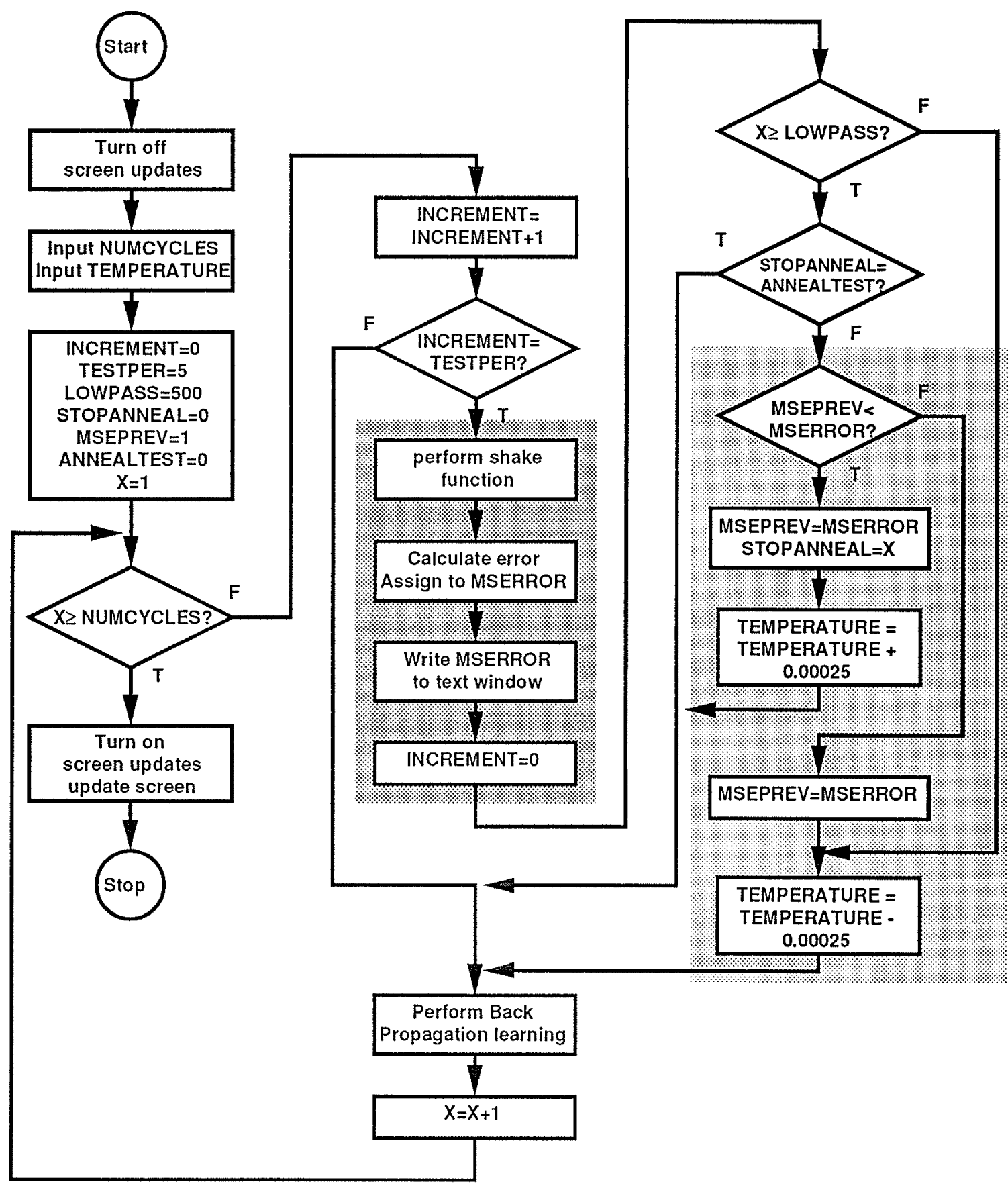

Mean Squared Error calculation section
Minimum Mean Squared Error test and annealing section

Figure 3.16:

Flowchart for the V2 Macro 
performed and annealing the network is also performed. The macro starts to test for the minimum MSE after the number of training cycles is greater than the variable IOWPASS, which was set to a high value so that the initial MSE fluctuations would not cause the annealing to stop prematurely. The variable MSEPREV holds the previous value for the MSE, which is compared to the latest value stored in MSERROR. If MSERROR is lower, then its value is written to MSEPREV, the temperature is lowered, and the learning continues. If MSERROR is higher, MSERROR is written to MSEPREV, the temperature is increased to the value where the minimum MSE was determined, STOPANNEAL is assigned the value of $X$, and learning continues. By assigning the value of $X$ to STOPANNEAL, the testing for minimum MSE is stopped at the point where STOPANNEAL is compared to ANNEALTEST.

- Performs various display housekeeping functions.

- Alerts the user when the simulation is complete.

The V3 Macro is the same as the V1 Nacro. In order to train the networks up to the point of the minimum NSL, the value of NUMCYCLES for each of the networks was set to the number of training cycles to reach minimum MSE found during the previous training using the V1 Macro. For the V2 and V3 network training, the following parameters were used:

- NUMCYCLES (for V2 Macro): 2000

- NUMCYCLES (for V3 Macro): See Table 3.6

- Initial temperature (TEMPERATURE): 0.2

- Learning rate: 0.2

- Momentum: 0.9

- Method of updating: Updating by group

- Learning rule: Back Propagation Batch with Momentum

By performing the training of the networks up to 2000 cycles, similar relationships were found between the V1, V2, and V3 Macro trained networks; the one exception to this was the 2.(16.6 networks, where the results for all 
macros were the same since the function did not reach a point of minimum MSE. For this network to exhibit the same relationship to the others tested, the number of training cycles performed has to exceed the number of cycles required to reach the minimum MSE. Since the minimum value was reached at 2265, the V1 and V2 networks were trained with the value of NUMCYCLES set to 2500 .

\subsection{Analysis of the V1, V2, and V3 Macro results}

A total of 9 network topologies using 3 different training methods were trained, resulting in a total of 27 trained noworks. These networks were then compared with respect to how well they learned the larget values of the training sets. A number of comparisons were performed on these networks, and these are described in this section.

\subsubsection{Analysis of the individual network results}

The Mean Squared Error value for each of the 27 trained networks were recorded; Table 3.7 compares these values for each of the macros tested. In all cases, the MSE values were lowest for the V2 Marro. This MSE value, however, does not give us any detailed information as to how the trained values compare to the target values of the training set. To provide a better analysis of the training, the set of trained output values was recorded for each network and compared to the training sets.

Table 3.7:

Comparison of MSt results for the Vl, V2, and V.3 Macros

\begin{tabular}{|c|c|c|c|}
\hline Network & V1 Macro MSE & V2 Macro MSE & V3 Macro MSE \\
\hline 2.06 .2 & 1.38996 E-2 & 2.12573 E-3 & 2.26890 E-3 \\
\hline 2.06 .4 & 5.58325 E-3 & 3.55090 E-3 & 4.41559 E-3 \\
\hline 2.06 .6 & 5.33428 E-2 & 4.79963 E-3 & 6.57322 E-3 \\
\hline 2.08 .2 & 1.21472 E-2 & 2.09814 E-3 & 2.25664 E-3 \\
\hline 2.08 .4 & 4.71355 E-2 & 2.83197 E-3 & 4.10530 E-3 \\
\hline 2.08 .6 & 4.47730 E-3 & 2.54631 E-3 & 3.41948 E-3 \\
\hline 2.10 .2 & 1.82580 E-2 & 2.14461 E-3 & 2.26328 E-3 \\
\hline 2.10 .4 & 5.22332 E-3 & 2.73264 E-3 & 4.26277 E-3 \\
\hline 2.10 .6 & 5.16670 E-3 & 3.56538 E-3 & 4.83774 E-3 \\
\hline
\end{tabular}


3.3.1.1 Average and standard deviation results for the trained networks

In addition to the Mean Squared Error results, more detailed results from each trained network were recorded for analysis. The table for one of the trained networks (Network V1.2.06.4) is shown in Table 3.8 for illustration, and the tables for all 27 of the trained networks are provided on pages B.1 to B.27 of Appendix B. Each table represents the results of a trained network, and provides the difference of the target (actual) values minus measured (trained) values of each output neuron for all input patterns. In addition, the following additional information was calculated for each output neuron and the whole network:

- The average of the absolute value of the difference of target minus measured output values. This value provides the average magnitude of the difference between measured and target values. The smaller the average, the closer the trained values are to the target values for the whole network.

- The standard deviation of the absolute value of the difference of target minus measured output values. This value provides a measure of the variation of the difference values for the trained network. The smaller the standard devialion, the more consistent the trained values are to the target values.

\subsubsection{Full and modified value results}

In analysing the results of the training, it was found that the networks performed differently with respect of the missing inpul/output vector pair representing the node being trained. In some of the networks, the learning process trained the outpul neurons w produce a \%ero value. This success in learning a zero value was dependent on the number of hidden layer neurons in each trained network; the neworks with 6 and 8 neurons per hidden layer learned smaller output values than the 10 neuron per hidden layer networks. 
Table 3.8

Training Resulls for V1.2.06.4

(Network 2.06.4, VI Macro)

\begin{tabular}{|c|c|c|c|c|c|c|}
\hline \multirow[t]{2}{*}{ Destination } & \multicolumn{3}{|c|}{ Neuron o1 (Route 5) } & \multicolumn{3}{|c|}{ Neuron 02 (Route 8) } \\
\hline & Target & Meas & Diff & Target & Meas & Diff \\
\hline$i 1$ & 0.3708 & 0.38696 & -0.0162 & 0.3838 & 0.34123 & 0.04257 \\
\hline i2 & 0.6027 & 0.59431 & 0.00839 & 0.4619 & 0.45203 & 0.00987 \\
\hline i3 & 0.5031 & 0.49084 & 0.01226 & 0.3623 & 0.34667 & 0.01563 \\
\hline 14 & 0.3088 & 0.2663 & 0.0425 & 0.2418 & 0.23695 & 0.00485 \\
\hline i5 & 0.213 & 0.28783 & -0.0748 & 0.3376 & 0.27743 & 0.06017 \\
\hline 16 & 0.5481 & 0.53388 & 0.01422 & 0.3776 & 0.36776 & 0.00984 \\
\hline$i 7$ & 0.4084 & 0.33077 & 0.0 & 0.2379 & 0.24452 & -0.0066 \\
\hline 18 & 0.4225 & 0.26476 & 0.15774 & 0.1281 & 0.2078 & -0.0797 \\
\hline 19 & & 0.03653 & -0.0365 & & 0.02367 & -0.0237 \\
\hline i10 & 0.6212 & 0.62441 & -0.0032 & 0.3761 & 0.37954 & -0.0034 \\
\hline$\$ 11$ & 0.5181 & 0.5127 & 0.0054 & 0.273 & 0.28933 & -0.0163 \\
\hline$\lceil 12$ & 0.5989 & 0.58511 & 0.01379 & 0.3045 & 0.31116 & -0.0067 \\
\hline$i 13$ & 0.7597 & 0.78442 & -0.0247 & 0.4653 & 0.46794 & -0.0026 \\
\hline 114 & 0.663 & 0.67004 & -0.007 & 0.4179 & 0.41063 & 0.00727 \\
\hline$\lceil 15$ & 0.6536 & 0.67837 & -0.0 & 0.4085 & 0.38525 & 0.02325 \\
\hline$i 16$ & 0.8046 & 0.79761 & 0.00 & 0.5102 & 0.4862 & 0.024 \\
\hline \multirow{4}{*}{$\begin{array}{l}\text { Neuron } \\
\text { Results }\end{array}$} & \multicolumn{2}{|c|}{ Average |Diff| (Full) } & 0.03289 & \multicolumn{2}{|c|}{ Average |Diff| (Full) } & 0.02103 \\
\hline & \multicolumn{2}{|c|}{ Average |Diff| (Mod) } & 0.03264 & \multicolumn{2}{|c|}{ Average |Diff| (Mod) } & 0.02086 \\
\hline & \multicolumn{2}{|c|}{\begin{tabular}{|l|l|l|l|} 
Std Dev |Diff| (Full) \\
Std
\end{tabular}} & 0.04048 & \multicolumn{2}{|c|}{ Std Dev [Diff] (Full) } & 0.02202 \\
\hline & \multicolumn{2}{|c|}{ Std Dev |Diff| (Mod) } & 0.04189 & \multicolumn{2}{|c|}{ Std Dev [Diff] (Mod) } & 0.02278 \\
\hline
\end{tabular}

\begin{tabular}{|c|c|c|c|c|c|c|}
\hline \multirow[t]{2}{*}{ Destination } & \multicolumn{3}{|c|}{ Neuron o3 (Route 12) } & \multicolumn{3}{|c|}{ Neuron o4 (Route 13) } \\
\hline & Target & Meas & Diff & Target & Meas & Diff \\
\hline$i 1$ & 0.5985 & 0.59328 & 0.00522 & 0.8306 & 0.83464 & -0.004 \\
\hline$i 2$ & 0.6191 & 0.61651 & 0.00259 & 0.8512 & 0.83803 & 0.01317 \\
\hline 13 & 0.5195 & 0.5021 & 0.0174 & 0.7516 & 0.76498 & -0.0134 \\
\hline$i 4$ & 0.4565 & 0.44198 & 0.01452 & 0.6886 & 0.70348 & -0.0149 \\
\hline ¡5 & 0.5523 & 0.53303 & 0.01927 & 0.7844 & 0.78615 & -0.0018 \\
\hline$i 6$ & 0.5072 & 0.49192 & 0.01528 & 0.7393 & 0.74378 & -0.0045 \\
\hline i7 & 0.395 & 0.38908 & 0.00592 & 0.6271 & 0.6 & -0.02 \\
\hline i8 & 0.3428 & 0.34296 & -0.0002 & 0.5749 & 0.58669 & -0.0118 \\
\hline i9 & & 0.02331 & -0.0233 & & 0.03318 & -0.0332 \\
\hline$\$ 10$ & 0.3884 & 0.38312 & 0.00528 & 0.6205 & 0.58901 & 0.03149 \\
\hline$i 11$ & 0.2853 & 0.2917 & -0.0064 & 0.5174 & 0.4 & 0.04227 \\
\hline$i 12$ & 0.1664 & 0.2295 & -0.0631 & 0.3985 & 0.31962 & 0.07888 \\
\hline$i 13$ & 0.3272 & 0.24207 & 0.08513 & 0.2377 & 0.24794 & -0.0102 \\
\hline$i-1$ & 0.3851 & 0.35 & -0.0054 & 0.6172 & 0.5 & 0.03275 \\
\hline & 0.2762 & 0.30626 & -0.0301 & 0.5083 & 0.4 & 0.05941 \\
\hline$i 16$ & 0.3712 & 0.33132 & 0.03988 & 0.3929 & 0.43999 & -0.0471 \\
\hline \multirow{4}{*}{$\begin{array}{l}\text { Neuron } \\
\text { Results }\end{array}$} & \multicolumn{2}{|c|}{ Average |Diff| (Full) } & 0.02118 & \multicolumn{2}{|c|}{ Average |Diff $\mid$ (Full) } & 0.02618 \\
\hline & \multicolumn{2}{|c|}{ Average |Diff| (Mod) } & 0.02104 & \multicolumn{2}{|c|}{ Average |Diff| (Mod) } & 0.02571 \\
\hline & \multicolumn{2}{|c|}{ Std Dev |Diff| (Full) } & 0.02362 & \multicolumn{2}{|c|}{ Std Dev |Diff| (Full) } & 0.02193 \\
\hline & \multicolumn{2}{|c|}{ Std Dev [Diff $\mid$ (Mod) } & 0.02445 & \multicolumn{2}{|c|}{ Std Dev |Diff] (Mod) } & 0.02262 \\
\hline
\end{tabular}

\begin{tabular}{|l|l|l|l|l|}
\hline $\begin{array}{l}\text { Network } \\
\text { Results }\end{array}$ & Average |Diff| (Full) & 0.02532 & Std Dev |Diff| (Full) & 0.02787 \\
\cline { 2 - 6 } & Average |Diff| (Mod) & 0.02506 & Std Dev |Diff| (Mod) & 0.02874 \\
\hline
\end{tabular}


In studying the difference values associated with the missing input/output vector, it was found that this difference value was significantly larger than the difference values for the other training patterns. In order to remove the effect of the missing input/output vector from the average and standard deviation values discussed above, modified average and standard deviation values were calculated that removed the missing input/output vector results. Both the full and modified average and standard deviation values for each output neuron and the whole network are provided for all of the trained networks. These values will be used in the comparisons that follow.

\subsubsection{Distribution of difference values}

The average and standard deviation values described above provide a basis for comparison of the target and measured outpul values. However, there was also interest in the distribution of the difference values for each model. The difference values for each model were analysed and the following distribution counts were determined:

- The number of target and measured values with an absolute difference of less than 0.01;

- The number of target and measured values with an absolute difference greater than or equal (0)(2.01 and less than (0.0)2; and

- The number of target and measured values with an absolute difference greater than of equal to 0.02 .

The values used for the coun thresholds were arbitrarily chosen based on a review of the difference values. By studying the counts listed above, we could study the distribution of the difference values for each network and compare these distributions against other networks; the better networks would display larger counts in the smaller difference ranges and smaller counts in larger difference ranges. The counts were also calculated using the full and modified results to study the effect of the originating node and the missing input/output vecur on these distributions. These counts will be used in the comparisons that follow. 


\subsubsection{Direct comparison of results between the different networks and macros}

For the following comparisons the average, standard deviation, and distribution counts were prepared separately for each network and then compared to other networks. These comparisons do not directly compare the difference values of networks using the same training sets against networks using different numbers of hidden neurons per layer or networks using different macros. To provide these direct comparisons, the following counts were performed:

- A set of three networks is compared, and the best result (smallest absolute difference) for carch masured outpul was determined and counted;

- After the best result for each measured outpul was determined, the difference results of the other networks were compared to it and counted if the difference between it and the best result was less than or equal to 0.01 ;

- After the best result for cach measured output was determined, the difference results of the other neworks were compared to it and counted if the difference between it and the best result was greater than 0.01 .

By determining the best results of sets of traned networks, a method of direct comparison between the various models under study is provided. Better networks will display larger counts of best results and smaller counts of result values that were worse than the best results. Poorer networks will display smaller counts of best results and larger counts of resull values that were worse than the best results. Tiwo of the following comparisons will compare sets of networks versus different numbers of hidden neurons and versus different macros used for training.

\subsubsection{Comparison $\# 1$ - description and results}

Table 3.9 contains nine comparisons of lained networks. In each comparison 
networks that were trained with the same macro and have the same number of output neurons were compared versus the number of neurons per hidden layer. For this comparison the difference distribution count for less than 0.01, the difference distribution count for greater than or equal to 0.02 , the average of the absolute value of the difference, and the standard deviation of the absolute value of the difference are compared. The best results in each comparison are shaded.

In analysing the results of this comparison, no single network topology $(6,8$, or 10 neurons per hidden layer) consistently provided the best results, but the 8 neuron per hidden layer topology performed hetter overall than the 6 or 10 neuron topologies by supplying the best results or close to the best results more often. The comparison of distribution counts showed that the originating node results consistently appcared in the highest count range, or the range where the absolute value of the difference was greater than or equal to 0.02 .

In the V1 Macro trained newworks, the difference in average and standard deviation values between the full and modified results was small for all network topologies. This showed thall these networks learned the zero value result for the untrained originaling node. In the V2 and V3 Macro comparisons, the modified average and standard deviation values were less than the values for the full set of results, with significant differences being found in the V2 and V3 Macro trained neworks with 2 and 4 output nodes. This shows that the $V 2$ and $V 3$ Macros did not learn the untrained originating node's values to the same degree as the V1 Narro.

\subsubsection{Comparison $\# 2$ - description and results}

Table 3.10 contains nine comparisons of trained networks. In each comparison networks that were trained with the same network topology (number of output neurons and number of neuron per hidden layer) were compared versus the macro used for training. For this comparison the difference distribution coun lor less than 0.01, the difference distribution 
Table 3.9

Comparison \#1:

Average/Standard Deviation measurements and distribution counts

Comparison versus number of neurons per hidden layer

\begin{tabular}{|c|c|c|c|c|c|c|c|c|c|c|}
\hline \multirow[t]{3}{*}{ NN Model } & \multicolumn{6}{|c|}{ Distribution Counts for $|\mathrm{X}|$} & \multirow{2}{*}{\multicolumn{2}{|c|}{$\begin{array}{l}\text { Average of } \\
\text { |Diff| }\end{array}$}} & \multirow{2}{*}{\multicolumn{2}{|c|}{$\begin{array}{l}\text { Std Deviation of } \\
\text { |Diff| }\end{array}$}} \\
\hline & \multicolumn{2}{|c|}{$|X|<.01$} & \multicolumn{2}{|c|}{$.01 \leq|X|<.02$} & \multicolumn{2}{|c|}{$|X| \geq .02$} & & & & \\
\hline & Full & Mod & Full & Mod & Full & Mod & Full & Mod & Full & Mod \\
\hline$V_{1.2 .06 .2}$ & 8 & 8 & 4 & 4 & 20 & 16 & 005003 & 0005358 & 0.06472 & 0.06688 \\
\hline$V 1.2 .08 .2$ & 4 & 4 & 6 & 6 & 22 & 20 & 0.05765 & 0.05653 & 00.007202 & 10006061 \\
\hline$V 1.2 .10 .2$ & 2 & 2 & 5 & 5 & 25 & 23 & 0.0747 & 0.07258 & 0.06829 & 0.07007 \\
\hline
\end{tabular}

\begin{tabular}{|c|c|c|c|c|c|c|c|c|c|c|}
\hline$V 1.2 .06 .4$ & 23 & 23 & 15 & 15 & 26 & 22 & 00253 & 0,02506 & 0.02787 & 0.02874 \\
\hline$V_{1.2 .08 .4}$ & 11 & 11 & 23 & 23 & 30 & 26 & 0.02687 & 0.02556 & 0002422 & 000248 \\
\hline$V_{1.2 .10 .4}$ & 13 & 13 & 18 & 18 & 33 & 29 & 0.0304 & 0.02779 & 0.02684 & 0.02491 \\
\hline
\end{tabular}

\begin{tabular}{|c|c|c|c|c|c|c|c|c|c|c|}
\hline $\mathrm{V} 1.2 .06 .6$ & 21 & 21 & 18 & 14 & 57 & 55 & 0.02638 & 0.02686 & 0.01845 & 0.01895 \\
\hline $\mathrm{V} 1.2 .08 .6$ & 27 & 27 & 24 & 22 & 45 & 41 & 0.02094 & 0.0209 & 0.00689 & 0.04 .488 \\
\hline $\mathrm{V} 1.2 .10 .6$ & 24 & 24 & 26 & 26 & 46 & 40 & 0.02261 & 0.02238 & 0.01867 & 0.01927 \\
\hline
\end{tabular}

\begin{tabular}{|c|c|c|c|c|c|c|c|c|c|c|}
\hline \multirow[t]{3}{*}{ NN Model } & \multicolumn{6}{|c|}{ Distribution Counts for $|X|$} & \multirow{2}{*}{\multicolumn{2}{|c|}{$\begin{array}{l}\text { Average of } \\
\text { |Diff| }\end{array}$}} & \multirow{2}{*}{\multicolumn{2}{|c|}{$\begin{array}{l}\text { Std Deviation of } \\
\text { |Diff } \mid\end{array}$}} \\
\hline & \multicolumn{2}{|c|}{$|x|<.01$} & \multicolumn{2}{|c|}{$01 \leq|X|<.02$} & \multicolumn{2}{|c|}{$|X| \geq .02$} & & & & \\
\hline & Full & Mod & Full & Mod & Full & Mod & Full & Mod & Full & Mod \\
\hline $\mathrm{V} 2.2 .06 .2$ & 12 & 12 & 8 & 8 & 12 & 90 & 0.04579 & 0.0251 & 0.08402 & 0.02116 \\
\hline$V 2.2 .08 .2$ & 11 & 11 & 6 & 6 & 15 & 13 & 0.04566 & 0002,182 & 0.08457 & 0.02117 \\
\hline $\mathrm{V} 2.2 .10 .2$ & 7 & 7 & 11 & 11 & 14 & 12 & 004545 & 0.0253 & 008106 & $002 / 15$ \\
\hline
\end{tabular}

\begin{tabular}{|c|c|c|c|c|c|c|c|c|c|c|}
\hline V2. & 22 & 22 & 19 & 19 & 23 & 19 & $03 \% 7$ & 0.02027 & 000456 & 0 \\
\hline $\mathrm{V} 2$ & 28 & 28 & 15 & 19 & 1 & 13 & & & & \\
\hline V2.2. & 29 & 29 & 3 & 15 & 20 & 16 & .04655 & 0.0 & 0.11968 & \\
\hline
\end{tabular}

\begin{tabular}{|c|c|c|c|c|c|c|c|c|c|c|}
\hline 2. & 21 & 21 & 19 & 15 & 56 & 54 & 0.02638 & 0.02686 & 0.01845 & 0018 \\
\hline$\overline{V 2}$ & 6 & 36 & 29 & 28 & 31 & 26 & & & & \\
\hline $\mathrm{V} 2.2$ & 35 & 35 & 2.5 & 22 & 39 & 33 & 0.02189 & 0.01787 & 0.02262 & .0 \\
\hline
\end{tabular}

\begin{tabular}{|c|c|c|c|c|c|c|c|c|c|c|}
\hline \multirow[t]{3}{*}{ NN Model } & \multicolumn{6}{|c|}{ Distribution Counts for $|X|$} & \multirow{2}{*}{\multicolumn{2}{|c|}{$\begin{array}{l}\text { Average of } \\
\text { |Diff| }\end{array}$}} & \multirow{2}{*}{\multicolumn{2}{|c|}{$\begin{array}{l}\text { Std Deviation of } \\
\text { |Diff | }\end{array}$}} \\
\hline & \multicolumn{2}{|c|}{$|X|<.01$} & \multicolumn{2}{|c|}{$.01 \leq|X|<.02$} & \multicolumn{2}{|c|}{$|x| \geq .02$} & & & & \\
\hline & Full & Mod & Full & Mod & Full & Mod & Full & Mod & Full & Mod \\
\hline V3.2.06.2 & 18 & 1 & 8 & 8 & 17 & 15 & 0.04698 & 0.02657 & 0.08294 & 0.02119 \\
\hline V3.2.08.2 & 7. & 7. & 9 & 9 & 16 & 14 & 0.047 & 0.02659 & 0.08293 & $0.02 \% 05$ \\
\hline V3.2.10.2 & 7 & 7 & 9 & 9 & 16 & 18 & 0.04622 & 0006614 & 0008058 & 0.02133 \\
\hline
\end{tabular}

\begin{tabular}{|c|c|c|c|c|c|c|c|c|c|c|}
\hline V3.2.06.4 & 23 & 23 & 12 & 12 & 20 & 25 & 0.040 .3 & 0.02358 & 0.07233 & 0.02412 \\
\hline V3.2.08.4 & 22 & 22 & 13 & 13 & 2.9 & 25 & 0.04388 & 0.02359 & 0.08596 & 0.02352 \\
\hline V3.2.10.4 & 22 & 22 & 12 & 12 & 30 & 26 & 0.05172 & 0.0237 & 0.11699 & 0.02366 \\
\hline
\end{tabular}

\begin{tabular}{|c|c|c|c|c|c|c|c|c|c|c|}
\hline V3 & 27 & 21 & 15 & 15 & 54 & 54 & 683 & 0.02826 & 0.20 .26 & 0.0201 \\
\hline & 86 & 06 & 27 & 27 & 33 & 27 & & & 0.03 & \\
\hline V3.2 & 20 & 26 & 20 & 20 & 50 & 44 & 0.02565 & 0.02215 & 0.02279 & 0.018 \\
\hline
\end{tabular}


Table 3.10

Comparison \#2:

Average/Standard Deviation measurements and distribution counts Comparison versus training macro

\begin{tabular}{|c|c|c|c|c|c|c|c|c|c|c|}
\hline \multirow[t]{3}{*}{ NN Model } & \multicolumn{6}{|c|}{ Distribution Counts for $|X|$} & \multirow{2}{*}{\multicolumn{2}{|c|}{$\begin{array}{l}\text { Average of } \\
\text { |Diff } \mid\end{array}$}} & \multirow{2}{*}{\multicolumn{2}{|c|}{$\begin{array}{l}\text { Std Deviation of } \\
\text { |Diff| }\end{array}$}} \\
\hline & \multicolumn{2}{|c|}{$|X|<.01$} & \multicolumn{2}{|c|}{$01 \leq|X|<.02$} & \multicolumn{2}{|c|}{$|x| \geq .02$} & & & & \\
\hline & Full & Mod & Full & Mod & Full & Mod & Full & Mod & Full & Mod \\
\hline$V 1.2 .06 .2$ & 8 & 8 & 4 & 4 & 20 & 18 & 0.05303 & 0.05358 & $0006 / 12$ & 0.06688 \\
\hline$V 2.2 .06 .2$ & 12 & 12 & 8 & 8 & $12 \%$ & 10: & $0.045 \%$ & 0.0251 & 0.08402 & $002 / 6$ \\
\hline V3.2.06.2 & 7 & 7 & 8 & 8 & 17 & 15 & 0.04698 & 0.02657 & 0.08294 & 0.02119 \\
\hline
\end{tabular}

\begin{tabular}{|c|c|c|c|c|c|c|c|c|c|c|}
\hline$V 1.2 .06 .4$ & 23 & 23 & 15 & 15 & 26 & 22 & 0.0253 & 0.02506 & 0.0 .218 & 0.02874 \\
\hline$V 2.2 .06 .4$ & 22 & 22 & 19 & 19 & 23 & 19 & 0.03777 & 0.02027 & 0.07456 & 0.02 .2020 \\
\hline$V 3.2 .06 .4$ & 2.3 & 23 & 12 & 12 & 29 & 25 & 0.04043 & 0.02358 & 0.07235 & 0.02412 \\
\hline
\end{tabular}

\begin{tabular}{|c|c|c|c|c|c|c|c|c|c|c|}
\hline$v 1.2 .06 .6$ & 21 & 21 & 18 & 14 & 57 & 55 & 0.608 & 00268 & 0.0189 & 895 \\
\hline V2. & 21 & 2 & 15 & 1 & 56 & 54 & & & & \\
\hline V3.2 & 27 & 21 & 15 & 15 & 54 & 54 & 0.02683 & 0.02826 & 0.02026 & 0.0201 \\
\hline
\end{tabular}

\begin{tabular}{|c|c|c|c|c|c|c|c|c|c|c|}
\hline \multirow[t]{3}{*}{ NN Model } & \multicolumn{6}{|c|}{ Distribution Counts for $|X|$} & \multirow{2}{*}{\multicolumn{2}{|c|}{$\begin{array}{l}\text { Average of } \\
\text { |Diff| }\end{array}$}} & \multirow{2}{*}{\multicolumn{2}{|c|}{$\begin{array}{l}\text { Std Deviation of } \\
\text { |Diff| }\end{array}$}} \\
\hline & \multicolumn{2}{|c|}{$|X|<.01$} & \multicolumn{2}{|c|}{$.01 \leq|X|<.02$} & \multicolumn{2}{|c|}{$|x| \geq .02$} & & & & \\
\hline & Full & Mod & Full & Mod & Full & Mod & Full & Mod & Full & Mod \\
\hline$V 1.2 .08 .2$ & 4 & 4 & 6 & 6 & 22 & 20 & 0.05765 & 0.05653 & 60.05782 & 0.05961 \\
\hline V2.2.08.2 & $1 \%$ & $17 \%$ & $\overline{6}$ & 6 & 15 & 18 & 0004566 & 10.62882 & 0.08457 & 0.02117 \\
\hline V3.2.08.2 & 7 & 7 & $\overline{9}$ & 9 & 16 & 14 & 0.047 & 0.02659 & 0.08293 & 0.02105 \\
\hline
\end{tabular}

\begin{tabular}{|c|c|c|c|c|c|c|c|c|c|c|}
\hline $\mathrm{V} 1.2$ & 11 & 11 & 23 & 23 & 30 & 26 & 0002687 & 0.02556 & 0.02422 & 0.02428 \\
\hline $\mathrm{V} 2.2$ & 28 & 28 & 19 & 19 & 17 & 13 & 0.03923 & $0.01 \% 9$ & 0.08956 & 100 \\
\hline V3.2.08.4 & 22 & 22 & 13 & 13 & 29 & 25 & 0.04388 & 0.02359 & 0.08596 & 0.02352 \\
\hline
\end{tabular}

\begin{tabular}{|c|c|c|c|c|c|c|c|c|c|c|}
\hline 11.2 .08 .6 & 27 & 27 & 24 & 22 & 45 & 41 & 0002094 & 0.02094 & 00 & 48 \\
\hline $\mathrm{V} 2.2$ & 36 & 36 & 29 & 28 & 3 & 26 & & & 94 & \\
\hline V3.2. & 36 & 36 & 27 & 27 & 33 & 27 & 0.02391 & 0.01752 & 0.03 & 0.0169 \\
\hline
\end{tabular}

\begin{tabular}{|c|c|c|c|c|c|c|c|c|c|c|}
\hline \multirow[t]{3}{*}{ NN Model } & \multicolumn{6}{|c|}{ Distribution Counts for $|X|$} & \multirow{2}{*}{\multicolumn{2}{|c|}{$\begin{array}{l}\text { Average of } \\
\text { |Diff } \mid\end{array}$}} & \multirow{2}{*}{\multicolumn{2}{|c|}{$\begin{array}{l}\text { Std Deviation of } \\
\text { |Diff } \mid\end{array}$}} \\
\hline & \multicolumn{2}{|c|}{$|x|<.01$} & \multicolumn{2}{|c|}{$.01 \leq|x|<.02$} & \multicolumn{2}{|c|}{$|x| \geq 02$} & & & & \\
\hline & Full & Mod & Full & Mod & Full & Mod & Full & Mod & Full & Mod \\
\hline $\mathrm{V} 1.2 .10 .2$ & 2 & 2 & 5 & 5 & 25 & 23 & 0.0747 & 0.07258 & 0.06829 & 0.07007 \\
\hline$V 2.2 .10 .2$ & 7 & 18 & 11 & 11 & 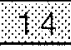 & $12 \%$ & 0001545 & 0.0253 & 0.08196 & 000215 \\
\hline V3.2.10.2 & 1 & 7 & 9 & 9 & 16 & 14 & 0.04622 & 0.02644 & 0.08058 & 0.02133 \\
\hline
\end{tabular}

\begin{tabular}{|c|c|c|c|c|c|c|c|c|c|c|}
\hline$V 1.2 .10 .4$ & 13 & 13 & 18 & 18 & 33 & 29 & 0.0304 & 0.02779 & 0.02688 & 0.02491 \\
\hline$V 2.2 .10 .4$ & 29 & 29 & 15 & 15 & 20 & 16 & 0.04655 & 0.01762 & 0.11968 & 0.0645 \\
\hline$V 3.2 .10 .4$ & 22 & 22 & 12 & 12 & 30 & 26 & 0.05172 & 0.0237 & 0.11699 & 0.02366 \\
\hline
\end{tabular}

\begin{tabular}{|c|c|c|c|c|c|c|c|c|c|c|}
\hline$V 1.2 .10 .6$ & 24 & 24 & 26 & 26 & 46 & 40 & 0.02261 & 0.02238 & 0.0 .0666 & 0.01927 \\
\hline$V 2.2 .10 .6$ & 35 & 35 & 22 & 22 & 39 & 33 & 0.02189 & $0.01 \% 8$ & 0.02262 & 0.0 .6682 \\
\hline$V 3.2 .10 .6$ & 26 & 26 & 20 & 20 & 50 & 44 & 0.02565 & 0.02215 & 0.02279 & 0.01883 \\
\hline
\end{tabular}


count for greater than or equal to 0.02 , the average of the absolute value of the difference, and the standard deviation of the absolute value of the difference are compared. The best results in each comparison are shaded.

In this comparison, the V2 Macro trained networks consistently performed better than the V1 and V3 Macro trained networks. This was most evident in the modified results, where the V2 trained networks provided the best result or tied with the best result in 34 of the 36 comparisons. This demonstrated that the V2 Macro trained networks performed the best learning of the target values.

\subsubsection{Comparison \#3 - description and results}

Table 3.11 contains nine comparisons of trained networks. Each comparison directly compared the measured output values of networks that were trained with the same macro and have the same number of output neurons versus the number of neurons per hidden layer. For this comparison the best result count, and the count for results greater than 0.01 than the best result were compared. The best results in each comparison are shaded.

The results of this comparison are very similar to those of Comparison \#1; no single network topology consistently produced the best results in learning the target values. Once again, the 8 neuron per hidden layer topology performed best overall, producing either the best or close to the best results in most of the comparisons.

\subsubsection{Comparison $\# 4$ - description and results}

Table 3.12 contains nine comparisons of trained networks. Each comparison directly compared the measured output values of networks that were trained with the same network topology (number of output neurons and number of neuron per hidden layer) versus the training macro used. For this 
Table 3.11

Comparison \#3:

Direct comparison of difference values

Comparison versus number of neurons per hidden layer

\begin{tabular}{|c|c|c|c|c|c|c|}
\hline \multirow[t]{3}{*}{ NN Model } & \multicolumn{6}{|c|}{ Counts - V1 Macro } \\
\hline & \multicolumn{2}{|c|}{ Best Result } & \multicolumn{2}{|c|}{ Result|SBest+.01 } & \multicolumn{2}{|c|}{ Result|>Best+.01 } \\
\hline & Full & Mod & Full & Mod & Full & Mod \\
\hline$V 1.2 .06 .2$ & 23. & 211 & 0 & 0 & 9 & 9 \\
\hline$V 1.2 .08 .2$ & 1 & 1 & 15 & 15 & 16 & 14 \\
\hline$V 1.2 .10 .2$ & 8 & 8 & 6 & $\overline{6}$ & 18 & 16 \\
\hline
\end{tabular}

\begin{tabular}{|c|c|c|c|c|c|c|}
\hline $\mathrm{V} 1.2 .06 .4$ & 29 & 2. & 20 & 20 & $1 / 5$. & 15 \\
\hline $\mathrm{V} 1.2 .08 .4$ & 19 & 19 & 28 & 27 & 17 & 14.2 \\
\hline $\mathrm{V} 1.2 .10 .4$ & 16 & 16 & 21 & 21 & 27 & 23 \\
\hline
\end{tabular}

\begin{tabular}{|c|c|c|c|c|c|c|}
\hline $\mathrm{V} 1.2 .06 .6$ & 26 & 21 & 25 & 24 & 45 & 45 \\
\hline $\mathrm{V} 1.2 .08 .6$ & 3.6 & 3.4 & 32 & 28 & 28 & 28.8 .8 \\
\hline $\mathrm{V} 1.2 .10 .6$ & 32 & 32 & 28 & 24 & 36 & 34 \\
\hline
\end{tabular}

\begin{tabular}{|c|c|c|c|c|c|c|}
\hline \multirow[t]{3}{*}{ NN Model } & \multicolumn{6}{|c|}{ Counts - V2 Macro } \\
\hline & \multicolumn{2}{|c|}{ Best Result } & \multicolumn{2}{|c|}{$\mid$ Result| $\leq$ Best+.01 } & \multicolumn{2}{|c|}{ Result|>Best+.01 } \\
\hline & Full & Mod & Full & Mod & Full & Mod \\
\hline$V 2.2 .06 .2$ & 8 & 8 & 24 & 22 & 0 & 0 \\
\hline V2.2.08.2 & 15 & 15 & 15 & 15 & 2 & 0 \\
\hline$V 2.2 .10 .2$ & 10 & 8 & 22 & 22 & 0 & 0 \\
\hline
\end{tabular}

\begin{tabular}{|c|c|c|c|c|c|c|}
\hline $\mathrm{V} 2.2 .06 .4$ & 16 & 12 & 36 & 36 & 12 & 12 \\
\hline $\mathrm{V} 2.2 .08 .4$ & 2.6 & 2.6 & 31 & 30 & 7 & 4 \\
\hline $\mathrm{V} 2.2 .10 .4$ & 22 & 22 & 37 & 37 & \% 5 5. \\
\hline
\end{tabular}

\begin{tabular}{|c|c|c|c|c|c|c|}
\hline $\mathrm{V} 2.2 .06 .6$ & 30 & 24 & 20 & 20 & 46 & 46 \\
\hline $\mathrm{V} 2.2 .08 .6$ & 3.8 & 38 & 30 & 30 & 28 & .22 .2 .1 \\
\hline $\mathrm{V} 2.2 .10 .6$ & 29 & 29 & 34 & 34 & 33 & 27 \\
\hline
\end{tabular}

\begin{tabular}{|c|c|c|c|c|c|c|}
\hline \multirow[t]{3}{*}{ NN Model } & \multicolumn{6}{|c|}{ Counts - V3 Macro } \\
\hline & \multicolumn{2}{|c|}{ Best Result } & \multicolumn{2}{|c|}{ Result $\mid \leq B e s t+.01$} & \multicolumn{2}{|c|}{ Result|>Best+.01 } \\
\hline & Full & Mod & Full & Mod & Full & Mod \\
\hline V3.2.06.2 & 10 & 10 & 19 & 18 & 3 & 2. \\
\hline V3.2.08.2 & 9 & 9 & 19 & 19 & 4 & 2 \\
\hline$\sqrt{ } 3.2 .10 .2$ & 18 & $\sqrt{2}$ & 16 & 16 & 2 & 2 \\
\hline
\end{tabular}

\begin{tabular}{|c|c|c|c|c|c|c|}
\hline V3.2.06.4 & 32 & 28 & 25 & 25 & 78 & 47 \\
\hline V3.2.08.4 & 15 & 15 & 38 & 37 & 11 & 8 \\
\hline V3.2.10.4 & 17 & 17 & 34 & 34 & $\overline{13}$ & 9 \\
\hline
\end{tabular}

\begin{tabular}{|c|c|c|c|c|c|c|}
\hline$V 3.2 .06 .6$ & 33 & 27 & 15 & 15 & 48 & 48 \\
\hline V3.2.08.6 & 4.4 & 4.8 & 27 & 27 & 25 & . \\
\hline V3.2.10.6 & 19 & 19 & 36 & 36 & 41 & 35 \\
\hline
\end{tabular}


Table 3.12

Comparison \#4:

Direct comparison of difference values

Comparison versus training macro

\begin{tabular}{|c|c|c|c|c|c|c|}
\hline \multirow[t]{3}{*}{ NN Model } & \multicolumn{6}{|c|}{ Counts - 6 neurons per hidden layer } \\
\hline & \multicolumn{2}{|c|}{ Best Result } & \multicolumn{2}{|c|}{ Result $\leq$ Best +.01} & \multicolumn{2}{|c|}{$\mid$ Result $\mid>$ Best +.01} \\
\hline & Full & Mod & Full & Mod & Full & Mod \\
\hline$V 1.2 .06 .2$ & 11 & 9 & 7 & 7 & 14 & 14 \\
\hline V2.2.06.2 & 94 & $1 \%$ & 11 & 11 & 7 & 58 \\
\hline V3.2.06.2 & 7 & 7 & 17 & 17 & 8 & 6 \\
\hline
\end{tabular}

\begin{tabular}{|c|c|c|c|c|c|c|}
\hline $\mathrm{V} 1.2 .06 .4$ & 28 & 24 & 20 & 20 & 1.6 .8 & 16 \\
\hline $\mathrm{V} 2.2 .06 .4$ & 26 & 2.6 & 22 & 22 & 1.6 .8 & .16 \\
\hline $\mathrm{V} 3.2 .06 .4$ & 10 & 10 & 30 & 30 & 24 & 20 \\
\hline
\end{tabular}

\begin{tabular}{|c|c|c|c|c|c|c|}
\hline$V 1.2 .06 .6$ & 51 & 5 & 24 & 24 & $2 \%$ & 15 \\
\hline V2.2.06.6 & 5 & 51 & 24 & 24 & $2 \%$ & 18 \\
\hline V3.2.06.6 & 45 & 39 & 25 & 25 & 26 & 26 \\
\hline
\end{tabular}

\begin{tabular}{|c|c|c|c|c|c|c|}
\hline \multirow[t]{3}{*}{ NN Model } & \multicolumn{6}{|c|}{ Counts - 8 neurons per hidden layer } \\
\hline & \multicolumn{2}{|c|}{ Best Result } & \multicolumn{2}{|c|}{ Result|sBest+.01 } & \multicolumn{2}{|c|}{$\mid$ Result $\mid>$ Best+. 01} \\
\hline & Full & Mod & Full & Mod & Full & Mod \\
\hline $\mathrm{V} 1.2 .08 .2$ & 10 & 8 & 3 & 3 & 19 & 19 \\
\hline V2.2.08.2 & 15. & 15. & 11 & 11 & 6. & (3. \\
\hline$\sqrt{ } 3.2 .08 .2$ & 8 & 8 & 15 & 15 & 9 & 7 \\
\hline
\end{tabular}

\begin{tabular}{|c|c|c|c|c|c|c|}
\hline$V 1.2 .08 .4$ & 20 & 16 & 17 & 17 & 27 & 27 \\
\hline$V 2.2 .08 .4$ & 27 & 27 & 23 & 23 & $1 \%$ & 180 \\
\hline V3.2.08.4 & 17 & 17 & 16 & 16 & 31 & 27 \\
\hline
\end{tabular}

\begin{tabular}{|c|c|c|c|c|c|c|}
\hline $\mathrm{V} 1.2 .08 .6$ & 20 & 14 & 44 & 44 & 32 & 32 \\
\hline $\mathrm{V} 2.2 .08 .6$ & 43 & .13 & 33 & 33 & 2.0 .8 & .3 .4 \\
\hline $\mathrm{V} 3.2 .08 .6$ & 34 & 34 & 33 & 33 & 29 & 23 \\
\hline
\end{tabular}

\begin{tabular}{|c|c|c|c|c|c|c|}
\hline \multirow[t]{3}{*}{ NN Model } & \multicolumn{6}{|c|}{ Counts -10 neurons per hidden layer } \\
\hline & \multicolumn{2}{|c|}{ Best Result } & \multicolumn{2}{|c|}{ Result $\mid \leq B e s t+.01$} & \multicolumn{2}{|c|}{ Result $\mid>$ Best +.01} \\
\hline & Full & Mod & Full & Mod & Full & Mod \\
\hline$V 1.2 .10 .2$ & 6 & 4 & 3 & 3 & 23 & 23 \\
\hline $\mathrm{V} 2.2 .10 .2$ & 15 & 45 & 12 & 12 & (3. & 3. \\
\hline V3.2.10.2 & 11 & 11 & 13 & 13 & 8 & 6 \\
\hline
\end{tabular}

\begin{tabular}{|c|c|c|c|c|c|c|}
\hline$V 1.2 .10 .4$ & 18 & 14 & 18 & 18 & 28 & 28 \\
\hline V2.2.10.4 & 32 & 32 & 20 & 20 & 112 & 88 \\
\hline V3.2.10.4 & 15 & 15 & 22 & 22 & 27 & 23 \\
\hline
\end{tabular}

\begin{tabular}{|c|c|c|c|c|c|c|}
\hline V1.2.10.6 & 32 & 26 & 30 & 30 & 34 & 34 \\
\hline V2.2.10.6 & 49 & .99 & 28 & 28 & 1.9 & .3. \\
\hline V3.2.10.6 & 16 & 16 & 40 & 40 & 40 & 34 \\
\hline
\end{tabular}


comparison the best result count, and the count for results greater than 0.01 than the best result were compared. The best results in each comparison are shaded.

The results of this comparison are very similar to those of Comparison \#2; in almost all cases, the V2 Macro trained networks produced better results than the V1 and V3 Macro trained networks. In comparing the modified results the V2 Macro provided the best results in all 18 comparisons.

\subsubsection{Summary of conclusions from Comparisons 1 to 4}

Comparisons \#2 and \#4 conclude that the V2 Macro trained networks produced the best trained networks of the three macros used in the simulations. If Comparisons \#1 and \#3 are revisited with the focus on the V2 Macro trained comparisons, analysis of these comparisons demonstrates that the 8 neuron per hidden layer network topology provided the best overall performance in learning the target values. In Comparison " 3 , the 8 neuron topology provided the best results in 5 of the 6 comparisons using the modified results. Similiarly, in Comparison \#1 the 8 neuron upology provided the best or near best results of the three topologies when comparing the full and modified difference distribution counts. In comparing the average and standard deviation values there was litle variation in the results of all three topologies, with the 8 neuron topology again producing the best or near best results.

Another conclusion from the comparisons was that it was useful to consider the effect of the originating node on the training of the network. The untrained node does affect the learning of the networks; at the end of this thesis some recommendations will be made for further research in this area.

Based on the comparisons performed above, networks using the 8 neuron per hidden layer topology and the $V 2$ Macro produced the best results for the range of output neurons configurations tested. 


\subsubsection{Comparison of the routing results of the trained networks}

In the comparisons performed above, the results of each trained network were judged as to how close the measured output values were to the target values. Our conclusions from these comparisons were that the V2 Macro trained networks performed best, and that the 8 node per hidden layer networks provided the best overall performance, though this result was not as clear as the macro comparisons. While this is an important part of this study, the ultimate goal of the research is to determine how well each network is able to perform a routing function. To perform a basic routing decision, the trained network is not required to exactly learn the target values; it is only required that the target values be learned to the degree that the ranking sequence of each output neuron in relation (o) the other output neurons can be determined. The next comparison evaluates the performance of the trained networks in determining these rankings.

As stated earlier, each output vector in a training set represents the routing information from an originating node to a particular destination node. The routing information consists of time based values, and each output neuron's value is compared or ranked against the other output values to determine the best route. If, as a result of training, the learned values are close enough to the actual values of the training set, then the routing sequences are learned and can be extracted from the network. Ilowever, if the difference between an actual and learned output value is sulficiently large, then this error can cause the output value to be larger or smaller than the learned output values of adjacent higher of lower ranked outputs, and a routing mismatch could occur.

Tables $3.13,3.14$, and 3.15 compare the routing results of V2 Macro trained networks for 2, 4, and 6 output nodes respectively: The routing results compare the performance of the neworks versus the number of neurons per hidden layer. The V2 Macro trained networks were chosen for comparison because of the performance of the $V 2$ Macro against the other macros in earlier comparisons. In each table the target rankings for each training set are provided, with rank 1 as the smallest value, rank 2 as the second smallest, up to 6 for the 6 output node networks. The masured rankings from each network 
Table 3.13:

Comparison of target rankings to trained rankings

of 2 output neuron networks using the V2 Macro.

\begin{tabular}{|c|c|}
\hline $\begin{array}{c}\text { Destination } \\
\text { Node }\end{array}$ \\
\hline$i 1$ \\
\hline$i 2$ \\
\hline$i 3$ \\
\hline$i 4$ \\
\hline$i 5$ \\
\hline$i 6$ \\
\hline 17 \\
\hline 18 Tanget \\
\hline 01 & 02 \\
\hline 1 & 2 \\
\hline 1 & \\
\hline 1 & 2 \\
\hline 1 & 2 \\
\hline 19 & 2 \\
\hline 2 & 1 \\
\hline 1 & 2 \\
\hline 1 & 2 \\
\hline 1 & 2 \\
\hline 110 \\
\hline 111 \\
\hline 112 \\
\hline 113 \\
\hline$i 14$ \\
\hline$i 15$ \\
\hline 116 \\
\hline 1 & 1 \\
\hline 1 & 2 \\
\hline 2 & 2 \\
\hline 2 & 1 \\
\hline 2 & 1 \\
\hline
\end{tabular}
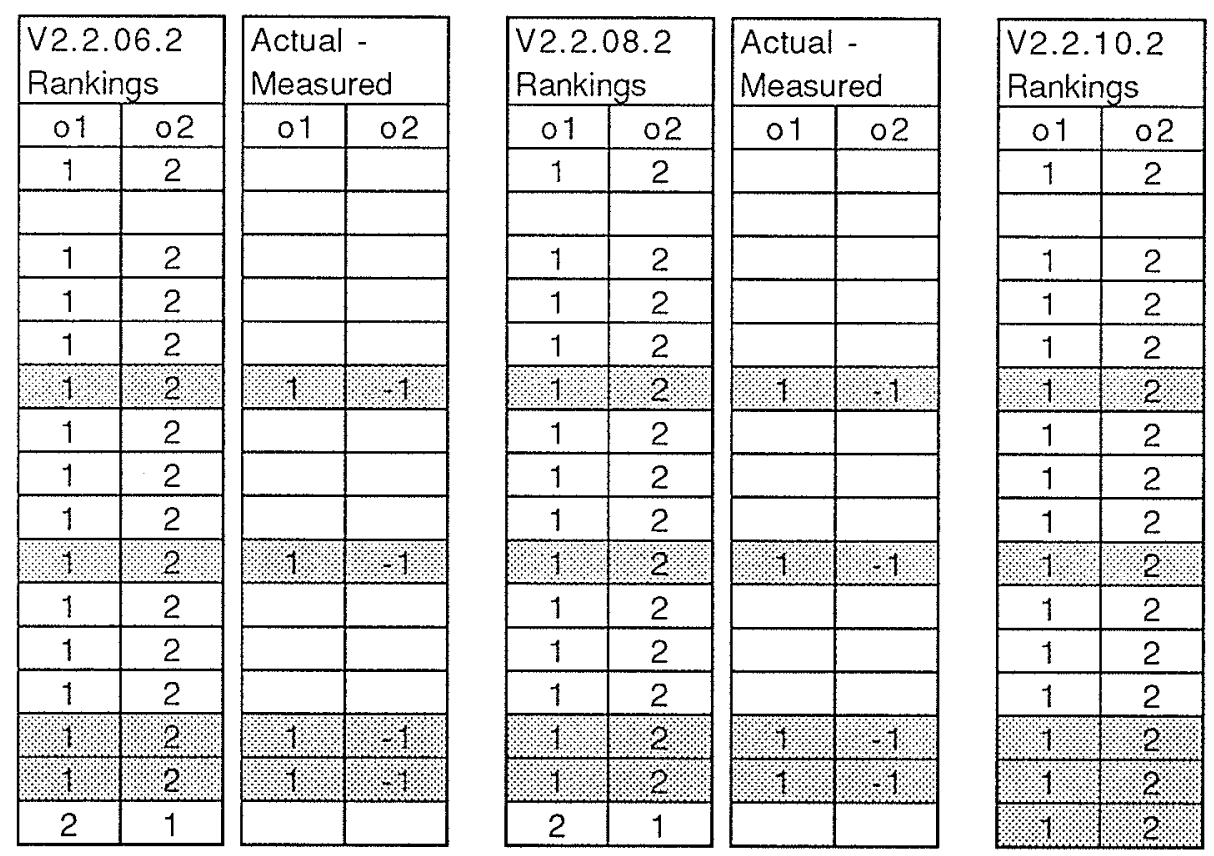

Actual Measured

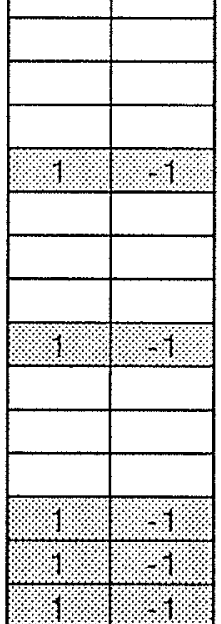


Table 3.14:

Comparison of target rankings to trained rankings for 4 output neuron networks using the V2 Macro

\begin{tabular}{|c|c|c|c|c|}
\hline \multirow[t]{2}{*}{$\begin{array}{c}\text { Destination } \\
\text { Node }\end{array}$} & \multicolumn{4}{|c|}{$\begin{array}{l}\text { Target } \\
\text { Rankings }\end{array}$} \\
\hline & 01 & 02 & 03 & 04 \\
\hline$i 1$ & 1 & 2 & 3 & 4 \\
\hline$i 2$ & 2 & 1 & 3 & 4 \\
\hline$i 3$ & 2 & 1 & 3 & 4 \\
\hline$\lcm{i 4}$ & 2 & 1 & 3 & 4 \\
\hline$i 5$ & 1 & 2 & 3 & 4 \\
\hline$i 6$ & 3 & 1 & 2 & 4 \\
\hline$i 7$ & 3 & 1 & 2 & 4 \\
\hline $\mathrm{i8}$ & 3 & 1 & 2 & 4 \\
\hline 19 & & & & \\
\hline 110 & 4 & 1 & 2 & 3 \\
\hline$i 11$ & 4 & 1 & 2 & 3 \\
\hline$i 12$ & 4 & 2 & 1 & 3 \\
\hline$i 13$ & 4 & 3 & 2 & 1 \\
\hline$i 14$ & 4 & 2 & 1 & 3 \\
\hline$i 15$ & 4 & 2 & 1 & 3 \\
\hline$i 16$ & 4 & 3 & 1 & 2 \\
\hline
\end{tabular}
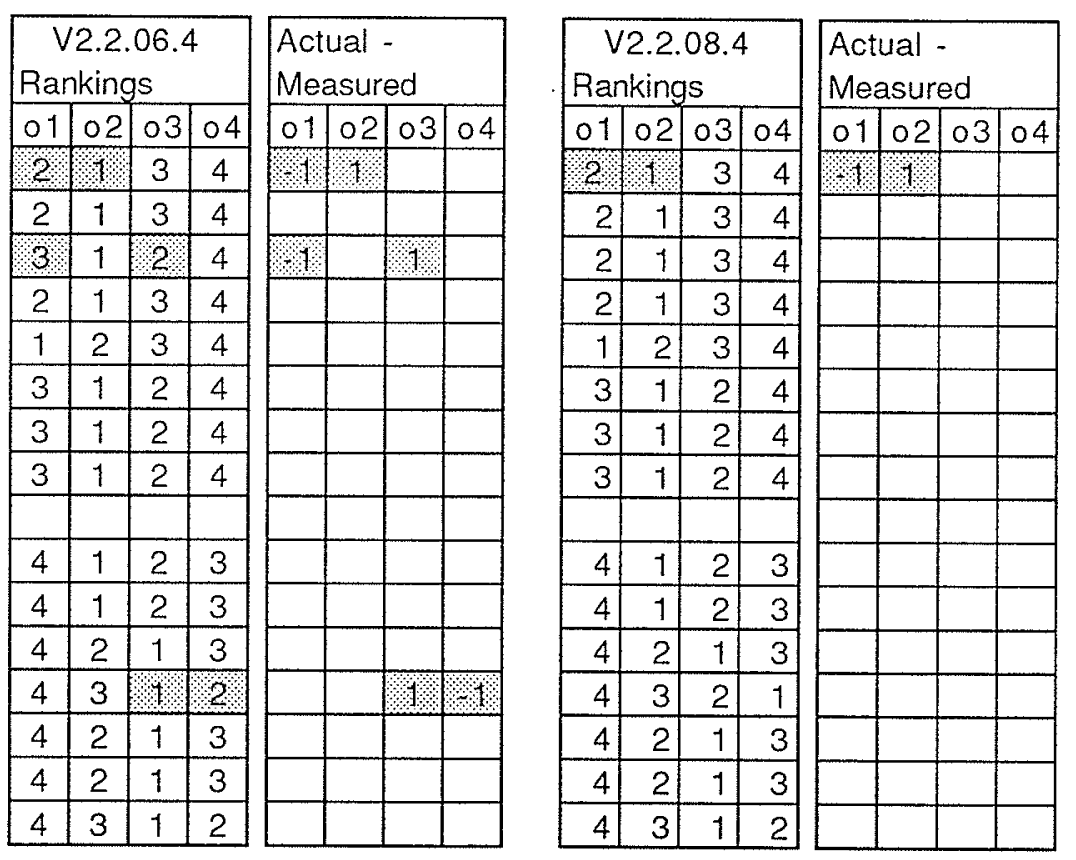

\begin{tabular}{|r|r|r|r|}
\hline \multicolumn{4}{|c|}{ V2.2.10.4 } \\
Rankings \\
\hline 01 & 02 & 03 & 04 \\
\hline 2 & 1 & 3 & 4 \\
\hline 2 & 1 & 3 & 4 \\
\hline 2 & 1 & 3 & 4 \\
\hline 2 & 1 & 3 & 4 \\
\hline 1 & 2 & 3 & 4 \\
\hline 3 & 1 & 2 & 4 \\
\hline 3 & 1 & 2 & 4 \\
\hline 3 & 1 & 2 & 4 \\
\hline & & & \\
\hline 4 & 1 & 2 & 3 \\
\hline 4 & 1 & 2 & 3 \\
\hline 4 & 2 & 1 & 3 \\
\hline 4 & 3 & 2 & 1 \\
\hline 4 & 2 & 1 & 3 \\
\hline 4 & 2 & 1 & 3 \\
\hline 4 & 3 & 1 & 2 \\
\hline
\end{tabular}

Actual -

Measured

\begin{tabular}{|l|l|l|l|}
\hline 01 & 02 & 03 & 04 \\
\hline
\end{tabular}

11

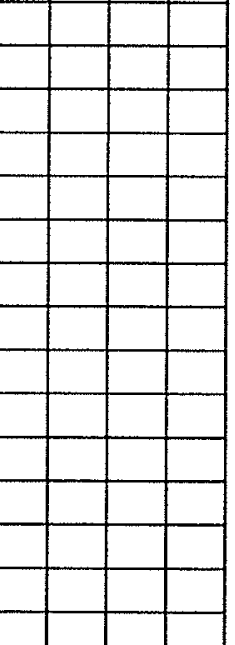


Table 3.15:

Comparison of target rankings to trained rankings

of 6 output neuron networks using the V2 Macro

\begin{tabular}{|c|c|c|c|c|c|c|}
\hline \multirow[t]{2}{*}{$\begin{array}{l}\text { Destination } \\
\text { Node }\end{array}$} & \multicolumn{6}{|c|}{$\begin{array}{l}\text { Target } \\
\text { Rankings }\end{array}$} \\
\hline & 01 & 02 & 03 & 104 & To5 & $\longdiv { 0 6 }$ \\
\hline$i 1$ & 1 & 2 & 3 & 4 & 5 & 6 \\
\hline i2 & 1 & 3 & 2 & 6 & 4 & 5 \\
\hline 13 & 1 & 3 & 2 & 5 & 4 & 6 \\
\hline$i 4$ & 1 & 2 & 4 & 3 & 6 & 5 \\
\hline$i 5$ & 1 & 2 & 4 & 3 & 6 & 5 \\
\hline$i 6$ & 2 & 4 & 1 & 6 & 3 & 5 \\
\hline 17 & 1 & 2 & 3 & 4 & 5 & 6 \\
\hline i8 & 2 & 1 & 5 & 3 & 6 & 4 \\
\hline 19 & 3 & 1 & 6 & 2 & 5 & 4 \\
\hline$i 10$ & 3 & 6 & 1 & 5 & 2 & 4 \\
\hline i11 & & & & & & \\
\hline $\mathrm{i} 12$ & 5 & 3 & 6 & 1 & 4 & 2 \\
\hline$i 13$ & 5 & 3 & 6 & \begin{tabular}{|l|}
1 \\
\end{tabular} & 4 & 2 \\
\hline$i 14$ & 5 & 6 & 2 & 4 & 1 & 3 \\
\hline $\mathrm{it5}$ & 6 & 5 & 4 & 2 & 3 & 1 \\
\hline$i 16$ & 6 & 5 & 4 & 2 & 3 & 1 \\
\hline
\end{tabular}
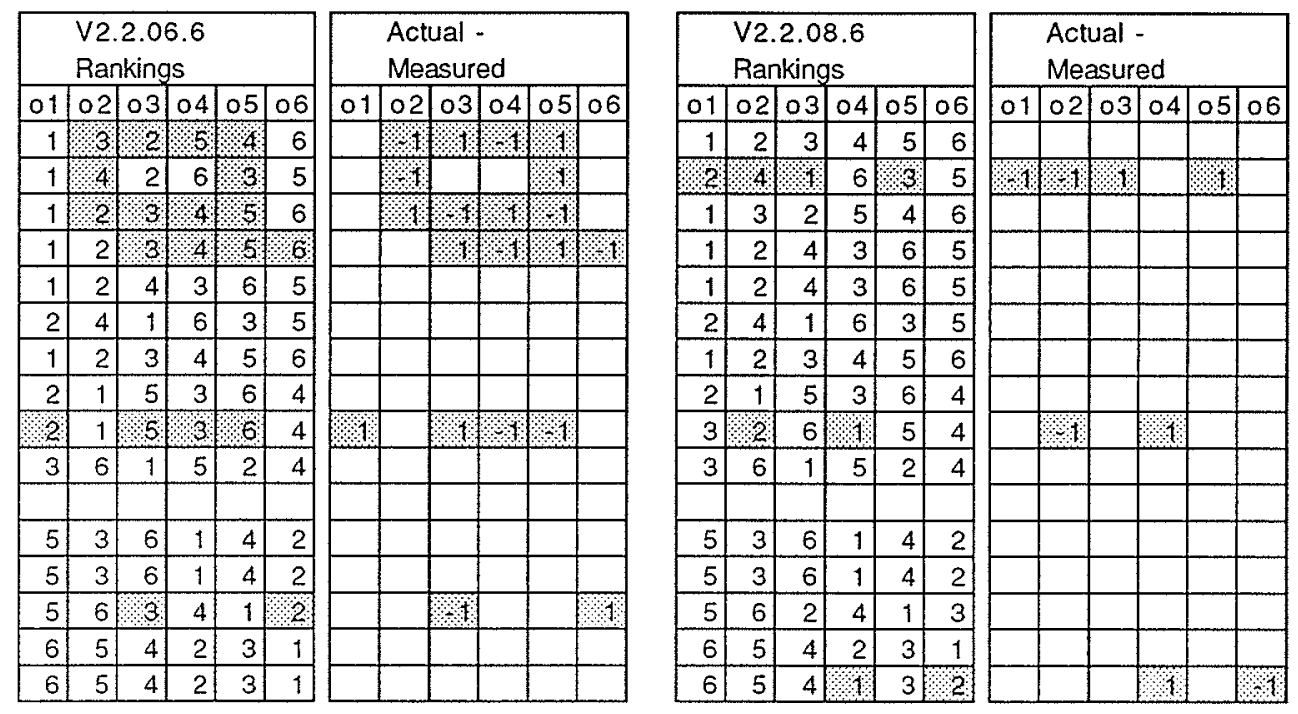

\begin{tabular}{|c|c|c|c|c|c|c|}
\hline $\begin{array}{c}\text { Destination } \\
\text { Node }\end{array}$ \\
\hline$i 1$ \\
\hline$i 2$ \\
\hline$i 3$ \\
\hline$i 4$ \\
\hline 15 \\
\hline 161 & 02 & 03 & 04 & 05 & 06 \\
\hline 1 & 2 & 3 & 4 & 5 & 6 \\
\hline 1 & 3 & 2 & 6 & 4 & 5 \\
\hline 1 & 3 & 2 & 5 & 4 & 6 \\
\hline 1 & 2 & 4 & 3 & 6 & 5 \\
\hline 1 & 2 & 4 & 3 & 6 & 5 \\
\hline 2 & 4 & 1 & 6 & 3 & 5 \\
\hline 1 & 2 & 3 & 4 & 5 & 6 \\
\hline 2 & 1 & 5 & 3 & 6 & 4 \\
\hline 3 & 1 & 6 & 2 & 5 & 4 \\
\hline 3 & 6 & 1 & 5 & 2 & 4 \\
\hline 18 \\
\hline$i 10$ \\
\hline$i 11$ \\
\hline 112 \\
\hline 5 & 3 & & & & \\
\hline 113 & 6 & 1 & 4 & 2 \\
\hline 114 & 3 & 6 & 1 & 4 & 2 \\
\hline 5 & 6 & 2 & 4 & 1 & 3 \\
\hline 6 & 5 & 4 & 2 & 3 & 1 \\
\hline 6 & 5 & 4 & 2 & 3 & 1 \\
\hline 115 &
\end{tabular}
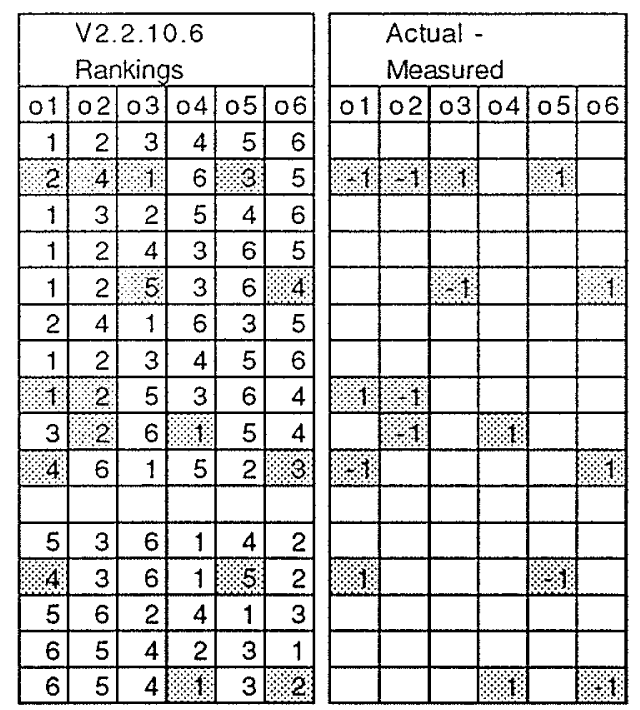
are also provided, and the difference of actual ranking versus measured ranking. No difference value (a difference of zero) means that the network learned the correct ranking for the output. If the difference is a positive value, the network learned an output ranking that is lower in value that the actual rank; if the difference is negative, the network learned an output ranking that is higher in value than the actual rank. The ranking mismatches and corresponding difference values are shaded.

In studying the results, it was found that all networks were trained to a level where the difference between measured and actual output ranking was never more than 1 or less than -1; this moans that all most a routing mismatch transposed the rankings of two outputs. In addition, the 8 neuron per hidden layer networks performed the best in learning the routing sequences, having the fewest mismatches in each comparison and overall. Table 3.16 provides a summary of routing performance for each network topology.

The results in the table agree with the general conclusions drawn from the previous comparisons. Based on these results, it was found that the 8 neuron per hidden layer networks using the V2 Nacro best performed the functions of learning training sets for rouling purposes.

Table 3.16:

Comparison of routing performance versus number of neurons per hidden layer

\begin{tabular}{|c|c|c|c|}
\hline Item & 6 neurons & 8 neurons & 10 neurons \\
\hline Mismatches - 2 outputs & 8 & 8 & 10 \\
\hline Mismatches - 4 outputs & 6 & 2 & 2 \\
\hline Mismatches - 6 outputs & 20 & 8 & 16 \\
\hline Total mismatches (out of 180 ) & 34 & 18 & 28 \\
\hline Total correct matches (out of 180 ) & 146 & 162 & 152 \\
\hline Percent correct matches & $81 \%$ & $90 \%$ & $84 \%$ \\
\hline
\end{tabular}

\subsubsection{Modification of the training set}

As demonstrated in the above results, the neural neworks are able to perform 
routing decisions with a $90 \%$ accuracy. If routing decisions were being performed strictly for the purposes determining a restoration route for a failed or lost link in a network, then the level of accuracy is higher. In the case of performing restoration routing, mismatchs between outputs with ranks 1 and 2 are not a concern; if a link with actual rank 1 and learned rank 2 fails and has to be restored, restoration would occur on a link that has an actual rank of 2 and learned rank of 1 . Despite the mismatch, the alternate link still has a lower ranking than the rest of the alternative links. If mismatches between rank 1 and 2 were ignored, the accuracy of the 8 neuron per hidden layer networks exceeds $99 \%$.

If, however, the network will be used (o perform more complex routing decisions than just restoralion, a higher level of accuracy is required. The work done to this point hats determined optimal parameters for both the network topology and the training macros; in order to further increase the accuracy, we must look at other factors. One of these is the training set.

In reviewing the training sets used, it was found that some output values in a given output vector were very close in value; this would represent links with almost equivalent routing times. If in the course of learning the target values the error in learning is grealter than the small difference between two close output values, then a routing mismatch would occur. If the difference between these target values were greater, then the effect of the errors produced by learning could be reduced. To test this statement, it was decided that modifying the training set should be tested.

There are several different ways thall the training set could be modified. One possible method is a pertorm further scaling of the data like that initially performed when creating the training sets. The problem with this approach, however, was that a complete training sel already used most of the range of values between 0 and 1 , and that the incremental benefit of scaling performed on the entire training set would be small. In order to provide better scaling, each output vector in the training set would have to be scaled separately. Scaling of each output vector would allow for greater separation of close output values, but even this may not be able on help in cases where the 
difference between target values can be as small as 0.001 .

An alternative approach to simple scaling would be to both scale and quantize the output values. In this case, the target values within each vector are ranked, and a quantized value is created for each value based on the ranking. By quantizing the output values, the real value separation of output values no longer affects the learning, and should result in fewer mismatches. There are several formulas that could be used to quantize these values, but the one decided upon was the following:

$$
\text { Quantizcdvalue }=\frac{1}{2 n}+\frac{r-1}{n}
$$

\section{Where}

$r \quad$ is the rank of output, and

$\mathrm{n} \quad$ is the number of output neurons (the node valence)

This formula provides a equal spacing of the larget values through the range of 0 and 1 of $1 / \mathrm{n}$, with the lowest and highest ranked values separated from 0 and 1 by $1 / 2 n$; this spacing from the ends of the value range results in the network not having (o learn training values at the extreme ends of the activation range. Table 3.17 provides a list of the quantization levels for different values of $n$.

Tabkes.t7:

Quancirect output values for different values of $n$

\begin{tabular}{|c|c|}
\hline$n$ & Quantized output levels \\
\hline 2 & $0.25,0.75$ \\
\hline 3 & $0.1666,0.5,0.8333$ \\
\hline 4 & $0.125,0.375,0.625,0.875$ \\
\hline 5 & $0.1,0.3,0.5,0.7,0.9$ \\
\hline 6 & $0.0833,0.25,0.4166,0.5833,0.75,0.9166$ \\
\hline
\end{tabular}




\subsubsection{Comparison of the initial and modified training set networks}

Using the formula above, the training sets used for the nework testing were modified; these modified training sets can be found on pages B.28 to B.30 of Appendix B. Neural networks consisting of 2, 4, and 6 output neurons and 8 neurons per hidden layer were trained using the modified training set, and the detailed tables of learning results were created; these tables can be found on pages B.31 to B.33 of Appendix B.

The learning performance of the modified training set networks was compared to the networks trained with the original training set. The six networks were compared in the following areas: mean squared error; average and standard deviation of the absolute value of the difference values; distribution of the absolute value of the difference values; and routing performance.

Table 3.18 shows the mean squared error results for the 6 networks. In this comparison, networks with $t$ and o output neurons had lower MSE values with the original traning set than the modilied training set networks, showing that these networks learned the real outpul values beter than the modified values. The 2 output neuron networts results were the reverse of the 4 and 6 output neurons results, showing a very high level of larning. In this case, the networks trained with the modilied values maly actually have memorized the output values, given the small number of output possibilities.

Table 3.18:

Comparison of MSF results for networks using original and modified training sets

\begin{tabular}{|c|c|c|}
\hline Network & MSE (original) & MSE (modified) \\
\hline V2.2.08.2 & $2.09814 E-3$ & $1.90260 E-5$ \\
\hline$V 2.2 .08 .4$ & $2.83197 E-3$ & $5.47008 E-3$ \\
\hline$V 2.2 .08 .6$ & $2.54631 E-3$ & $1.372125 E-2$ \\
\hline
\end{tabular}

Table 3.19 shows the comparison ol the dilference distribution count for less 
than 0.01 , the difference distribution count for greater than or equal to 0.02 , the average of the absolute value of the difference, and the standard deviation of the absolute value of the difference between networks trained with the original and modified training sets. These comparisons show that for the 2 output neuron networks the modified training produced very precise learning, but for the larger networks the original training set produced better results. This comparison agrees with the MSE results found above.

Table 3.19:

Neural Network Model Test Results:

Average/Standard Deviation measurements and distribution counts Comparison of $V 2$ traned neworks using real and modified training sets

\begin{tabular}{|c|c|c|c|c|c|c|c|c|c|c|}
\hline \multirow[t]{3}{*}{ NN Model } & \multicolumn{6}{|c|}{ Distribution Counts for $|\mathrm{X}|$} & \multirow{2}{*}{\multicolumn{2}{|c|}{$\begin{array}{c}\text { Average of } \\
\text { |Diff } \mid\end{array}$}} & \multirow{2}{*}{\multicolumn{2}{|c|}{$\begin{array}{l}\text { Std Deviation of } \\
\text { |Diff } \mid\end{array}$}} \\
\hline & \multicolumn{2}{|c|}{$x<.01$} & \multicolumn{2}{|c|}{$.01 \leq X<.02$} & \multicolumn{2}{|c|}{$x \geq .02$} & & & & \\
\hline & Full & Mod & Full & Mod & Full & Mod & Full & Mod & Full & Mod \\
\hline $\mathrm{V} 2.2$ & 11 & 11 & 6 & 6 & 15 & 13 & 0.04566 & 0.02482 & 00845 & 0.02117 \\
\hline $\mathrm{V} 2.08 .2(\mathrm{M})$ & 30 & 30 & 0 & 0 & 2 & 0 & 0.02928 & 000236 & 0.10592 & 000021 \\
\hline
\end{tabular}

\begin{tabular}{|c|c|c|c|c|c|c|c|c|c|c|}
\hline $\mathrm{V} 2.2 .08 .4$ & 28 & 28 & 19 & 19 & 17 & 13 & 0.03923 & 0.0179 & 0.08956 & 0.0210 .2 \\
\hline $\mathrm{V} 2.2 .08 .4(\mathrm{M})$ & 17 & 17 & 15 & 15 & 32 & 28 & 0.03248 & 0.0277 & 0.0330 & 0.0247 \\
\hline
\end{tabular}

\begin{tabular}{|c|c|c|c|c|c|c|c|c|c|c|}
\hline $\mathrm{V} 2.2 .10 .6$ & 36 & 36 & 29 & 28 & 31 & 26 & 0.02253 & 0.01569 & 0.02994 & 0.01354 \\
\hline $\mathrm{V} 2.2 .10 .6(\mathrm{M})$ & 9 & 9 & 13 & 13 & 74 & 68 & 0.04062 & 0.04084 & 0.02444 & 0.02509 \\
\hline
\end{tabular}

The comparisons made above show that the original training set networks performed better at learning the outpul values of the training sets. But how do the networks compare in the learning of the rankings of the outputs? Tables $3.20,3.21$, and 3.22 compare the routing results of the original and modified training set networks. These comparisons show that the modified training set networks learned the routing rankings better than the original training set, producing only 2 routing mismalches oul of 180 output rankings. The probable reason for the improved performance in ranking is due to the scaling and quantizing of target output values; the original training set networks learned the actual values belce, but the separation of the output values in the modilied training sets was latger than the learning errors introduced by modifying the values. 


\subsection{Conclusions from single network node modelling}

Based on the limited amount of testing performed, the conclusions made from the research are as follows:

- The four layer feed forward network, back propagation learning rule, and continuous sigmoidal function were appropriate testing choices for the learning lask.

- A learning process involving annealing of the network until reaching minimum mean squared error, followed by further learning with the temperature held constant, provided the best learning results.

- 8 neuron per hidden layer networks performed best overall when applied to learning tasks involving networks with a range of 2 to 6 output neurons

- Scaling and quantizing of output values in the training sets produced networks that perform routing decisions better than networks with unscaled and unquantized values. 
Table 3.20:

V2 Macro 2 Output Neuron Routing Comparison - real vs. modified training set

\begin{tabular}{|r|r|}
\hline $\begin{array}{c}\text { Destination } \\
\text { Node }\end{array}$ & \multicolumn{2}{|c|}{$\begin{array}{l}\text { Target } \\
\text { Rankings }\end{array}$} \\
\hline 01 & 02 \\
\hline 11 \\
\hline 12 \\
\hline 13 \\
\hline 14 \\
\hline$i 5$ \\
\hline 16 \\
\hline 17 \\
\hline 1 & 2 \\
\hline 18 \\
\hline 19 \\
\hline 110 \\
\hline 111 \\
\hline 112 \\
\hline 113 \\
\hline 114 \\
\hline 115 \\
\hline 116 \\
\hline 1 & 2 \\
\hline 1 & 2 \\
\hline 1 & 2 \\
\hline 1 & 2 \\
\hline 2 & 1 \\
\hline 2 & 1 \\
\hline 2 & 1 \\
\hline
\end{tabular}
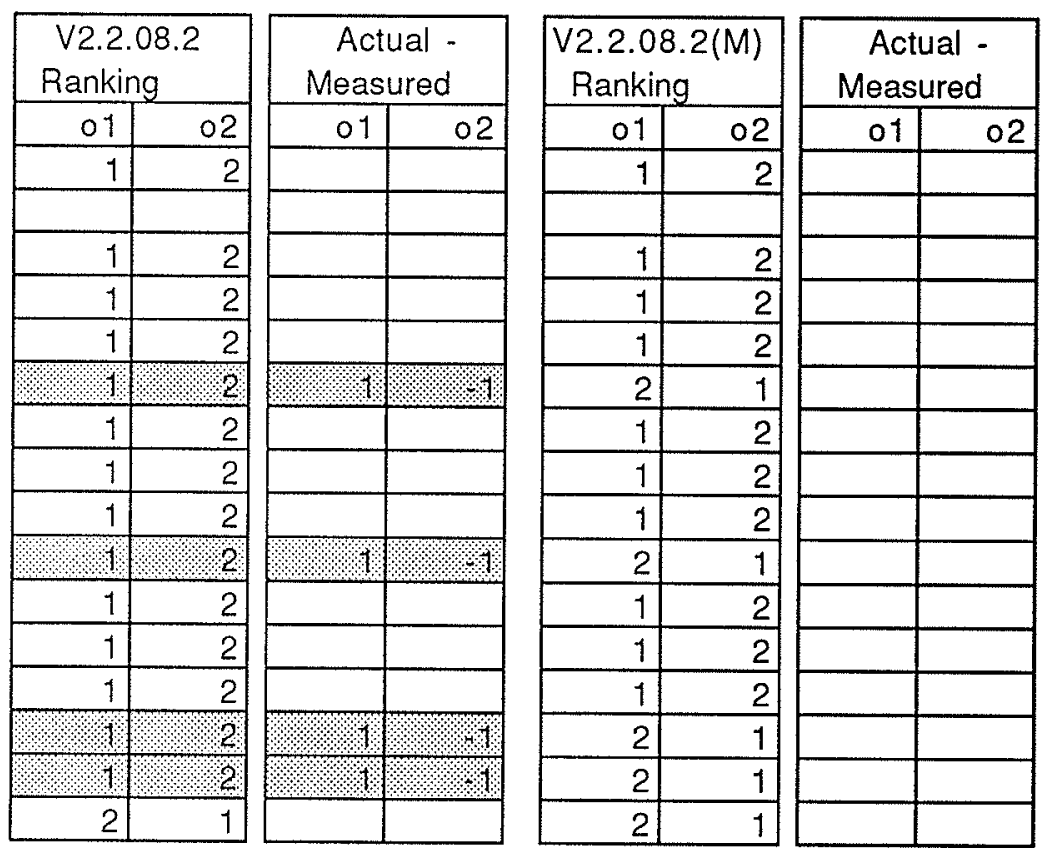

Table 3.21:

V2 Macro 4 Output Neuron Routing Comparison - real vs. modified training set

\begin{tabular}{|c|c|c|c|c|c|c|c|c|c|c|c|c|c|c|c|c|c|c|c|c|}
\hline \multirow[t]{2}{*}{$\begin{array}{l}\text { Destination } \\
\text { Node }\end{array}$} & \multicolumn{4}{|c|}{$\begin{array}{l}\text { Target } \\
\text { Rankings }\end{array}$} & \multicolumn{4}{|c|}{$\begin{array}{l}\text { V2.2.08.4 } \\
\text { Ranking }\end{array}$} & \multicolumn{4}{|c|}{$\begin{array}{r}\text { Actual - } \\
\text { Measured }\end{array}$} & \multicolumn{4}{|c|}{\begin{tabular}{|l|} 
V2.2.08.4(M) \\
Ranking \\
\end{tabular}} & \multicolumn{4}{|c|}{$\begin{array}{r}\text { Actual - } \\
\text { Measured }\end{array}$} \\
\hline & 01 & 02 & 03 & 04 & 01 & 02 & 03 & 04 & 01 & 02 & o3 & 04 & 01 & 02 & 03 & 04 & 01 & 02 & 03 & 04 \\
\hline i1 & 1 & 2 & 3 & 4 & 2 & 1 & 3 & 4 & 3 & 1 & & & 1 & 2 & 3 & 4 & & & & \\
\hline$i 2$ & 2 & 1 & 3 & 4 & 2 & 1 & 3 & 4 & & & & & 2 & 1 & 3 & 4 & & & & \\
\hline i3 & 2 & 1 & 3 & 4 & 2 & 1 & 3 & 4 & & & & & 2 & 1 & 3 & 4 & & & & \\
\hline 14 & 2 & 1 & 3 & 4 & 2 & 1 & 3 & 4 & & & & & 2 & 1 & 3 & 4 & & & & \\
\hline$i 5$ & 1 & 2 & 3 & 4 & 1 & 2 & 3 & 4 & & & & & 1 & 2 & 3 & 4 & & & & \\
\hline i6 & 3 & 1 & 2 & 4 & 3 & 1 & 2 & 4 & & & & & 3 & 1 & 2 & 4 & & & & \\
\hline 17 & 3 & 1 & 2 & 4 & 3 & 1 & 2 & 4 & & & & & 3 & 1 & 2 & 4 & & & & \\
\hline$i 8$ & 3 & 1 & 2 & 4 & 3 & 1 & 2 & 4 & & & & & 3 & 1 & 2 & 4 & & & & \\
\hline 19 & & & & & & & & & & & & & & & & & & & & \\
\hline 110 & 4 & 1 & 2 & 3 & 4 & 1 & 2 & 3 & & & & & 4 & 1 & 2 & 3 & & & & \\
\hline$i 11$ & 4 & 1 & 2 & 3 & 4 & 1 & 2 & 3 & & & & & 4 & 1 & 2 & 3 & & & & \\
\hline$i 12$ & 4 & 2 & 1 & 3 & 4 & 2 & 1 & 3 & & & & & 4 & 2 & 1 & 3 & & & & \\
\hline$i 13$ & 4 & 3 & 2 & 1 & 4 & 3 & 2 & 1 & & & & & 4 & 3 & 2 & 1 & & & & \\
\hline$i 14$ & 4 & 2 & 1 & 3 & 4 & 2 & 1 & 3 & & & & & 4 & 2 & 1 & 3 & & & & \\
\hline$i 15$ & 4 & 2 & 1 & 3 & 4 & 2 & 1 & 3 & & & & & 4 & 2 & 1 & 3 & & & & \\
\hline$i 16$ & 4 & 3 & 1 & 2 & 4 & 3 & 1 & 2 & & & & & 4 & 3 & 1 & 2 & & & & \\
\hline
\end{tabular}


Table 3.22:

V2 Macro 6 Output Neuron Routing Comparison - real vs. modified training set

\begin{tabular}{|c|c|c|c|c|c|c|}
\hline \multirow[t]{2}{*}{$\begin{array}{l}\text { Dest } \\
\text { Node }\end{array}$} & \multicolumn{6}{|c|}{$\begin{array}{l}\text { Target } \\
\text { Rankings }\end{array}$} \\
\hline & \begin{tabular}{|l|}
01 \\
\end{tabular} & 02 & 03 & 04 & 05 & $\longdiv { 0 6 }$ \\
\hline$i 1$ & 1 & 2 & 3 & 4 & 5 & 6 \\
\hline i2 & 1 & 3 & 2 & 6 & 4 & 5 \\
\hline i3 & 1 & 3 & 2 & 5 & 4 & 6 \\
\hline$i 4$ & 1 & 2 & 4 & 3 & 6 & 5 \\
\hline$i 5$ & 1 & 2 & 4 & 3 & 6 & 5 \\
\hline i6 & 2 & 4 & 1 & 6 & 3 & 5 \\
\hline i7 & 1 & 2 & 3 & 4 & 5 & 6 \\
\hline$i 8$ & 2 & 1 & 5 & 3 & 6 & 4 \\
\hline 19 & 3 & 1 & 6 & 2 & 5 & 4 \\
\hline$i 10$ & 3 & 6 & 1 & 5 & 2 & 4 \\
\hline $\mathrm{i11}$ & & & & & & \\
\hline$i 12$ & 5 & 3 & 6 & 1 & 4 & 2 \\
\hline $\mathrm{i} 13$ & 5 & 3 & 6 & 1 & 4 & 2 \\
\hline$i 14$ & 5 & 6 & 2 & 4 & 1 & 3 \\
\hline 115 & 6 & 5 & 4 & 2 & 3 & 1 \\
\hline$i 16$ & 6 & 5 & 4 & 2 & 3 & 1 \\
\hline
\end{tabular}
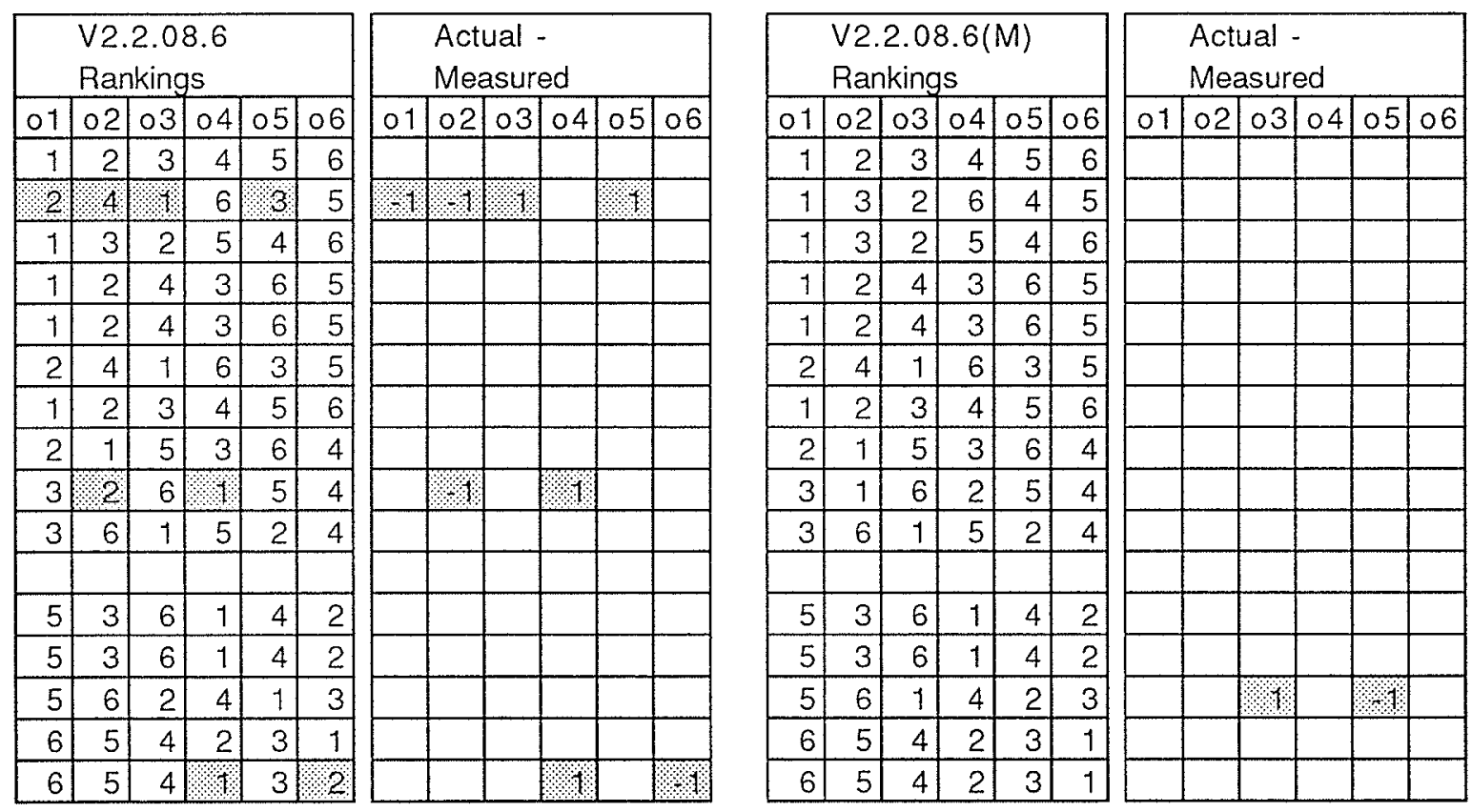


\section{Chapter 4}

\section{Full Network Modelling}

In this chapter the neural network topology, learning method, and modified training set identified in the previous chapter are applied to the full sample network, and the routing results determined. The neural networks are then tested to evaluate their ability to perform routing decisions related to different routing scenarios common in telecommunications networks. The chapter concludes with testing of the ability of the trained neural networks to perform generalized routing decisions, and a discussion of the results.

\subsection{Full network simulation results}

Using the findings of the single node research, the remaining 13 nodes of the sample network were simulated to study the routing performance of the trained networks for the entire sample network. For each network node the following activities were performed:

- The routing information and routing rankings for each node was determined.

- The ranking information was used to create the modified training set based on the formula used in the single node modelling.

- A neural network was created with 8 neurons per hidden layer and the required number of neurons for the output layer.

- The neural network was trained using the V2 Macro. The settings used for training were as per the single node research, but the number of training cycles was increased to 3000 to ensure the networks would have enough cycles to reach the point where the annealing process is stopped.

- The output values of the trained networks were recovered and the output rankings determined.

- The target rankings and measured rankings were compared. 
Table 4.1 shows the information and results determined in the simulation of node 12 of the sample network. The results for all network nodes can be found on pages C. 1 to C. 16 of Appendix C. The upper half of Table 4.1 show the actual routing values, the target rankings derived from this routing information, and the target training values used for the simulation. The bottom half of Table 4.1 shows the measured values recovered after simulation, and measured rankings produced from the values, and the comparison of actual and measured rankings. Routing mismatches have been shaded for presentation purposes to highlight their occurrence. A review of the sample network results shows that eight routing mismatches occurred out of 990 routing rankings. This performance translates into a routing accuracy of $99.19 \%$ for the overall network. Two pairs of routing mismatches occurred in Sample Network nodes with 5 links (one each in Nodes 6 and 7) and two pairs of routing mismatches occurred in sample network nodes with 6 links (one each in Nodes 11 and 12).

The occurrence of the routing mismatches suggests that neural networks with larger number of output neurons are more susceptible to routing mismatches; these networks have smaller quantization steps between the modified output values, and would be the more sensitive to mismatches caused by large learning errors. In addition, the mismatches occurred with the highest ranked or lowest ranked outputs, at the extremes of the output value range. This suggests that the network had difficulty learning the target values at the extremes of the value range, and a review of the measured values shows large errors in the mismatched outputs that resulted in the transposition of the output rankings. No routing mismatches occurred in sample network nodes with 4 or less links; Table 4.2 provides the details of the routing performance for the complete Sample Network and for the sets of nodes with equal numbers of links.

\subsection{Performing routing decisions}

When performing a routing decision, the goal is to select the best route that meets the routing criteria. The criteria used throughout this thesis has been 
Table 4.1:

Routing Results for Node 12 of the Sample Network
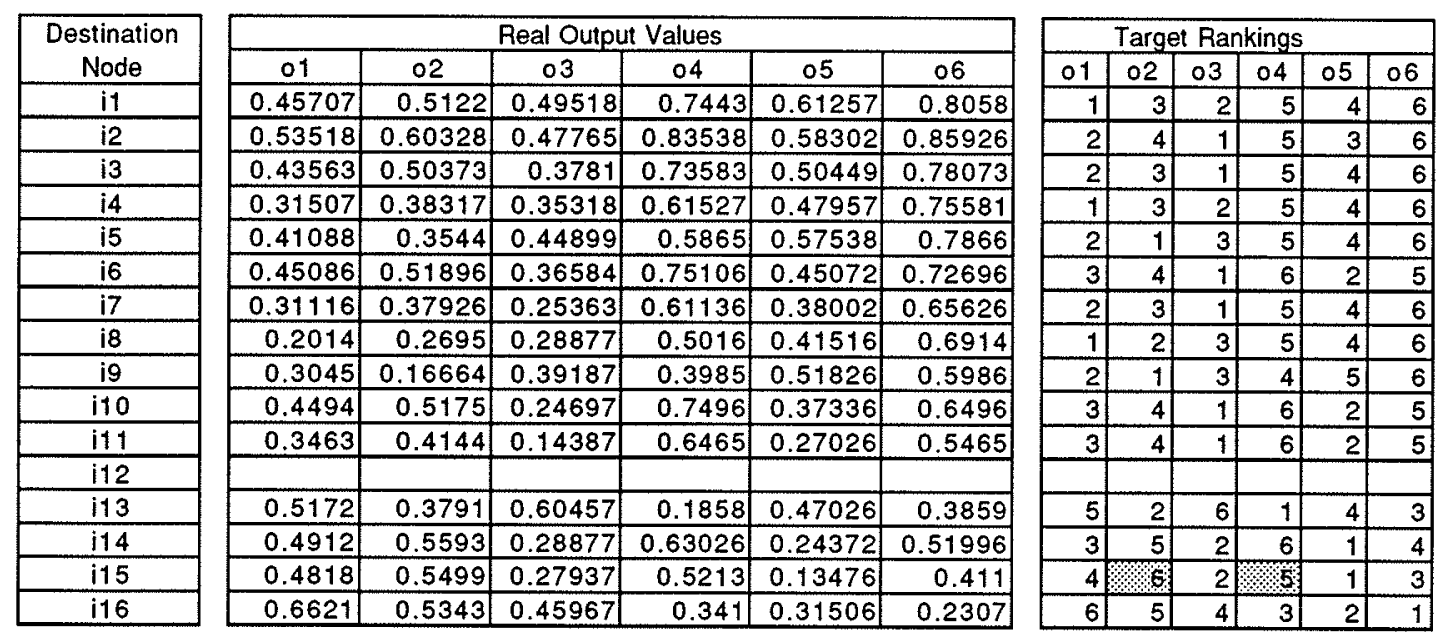

\begin{tabular}{|c|}
\hline $\begin{array}{c}\text { Destination } \\
\text { Node }\end{array}$ \\
\hline$i 1$ \\
\hline$i 2$ \\
\hline$i 3$ \\
\hline$i 4$ \\
\hline$i 5$ \\
\hline$i 6$ \\
\hline$i 7$ \\
\hline$i 8$ \\
\hline$i 9$ \\
\hline 110 \\
\hline$i 11$ \\
\hline 112 \\
\hline 113 \\
\hline$i 14$ \\
\hline$i 15$ \\
\hline 116 \\
\hline
\end{tabular}
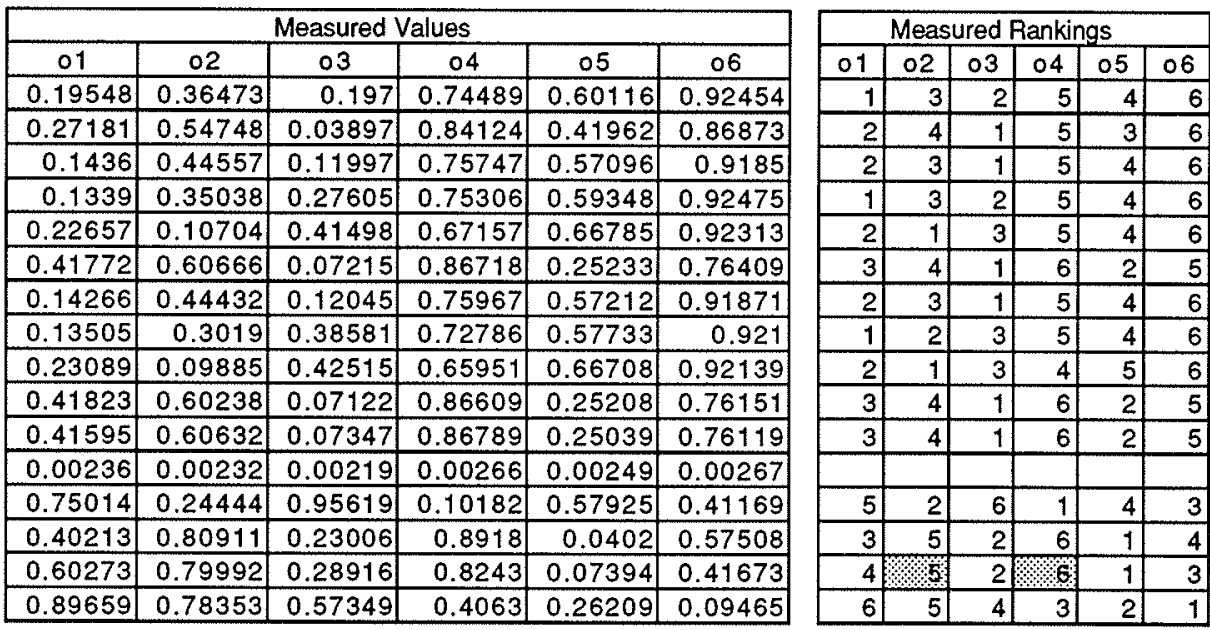

\begin{tabular}{|r|c|r|r|r|r|}
\hline \multicolumn{7}{|c|}{ Target Values } \\
\hline 01 & \multicolumn{1}{|c|}{02} & \multicolumn{1}{|c|}{03} & \multicolumn{1}{c|}{04} & \multicolumn{1}{c|}{05} & \multicolumn{1}{c|}{06} \\
\hline 0.08333 & 0.41667 & 0.25 & 0.75 & 0.58333 & 0.91667 \\
\hline 0.25 & 0.58333 & 0.08333 & 0.75 & 0.41667 & 0.91667 \\
\hline 0.25 & 0.41667 & 0.08333 & 0.75 & 0.58333 & 0.91667 \\
\hline 0.08333 & 0.41667 & 0.25 & 0.75 & 0.58333 & 0.91667 \\
\hline 0.25 & 0.08333 & 0.41667 & 0.75 & 0.58333 & 0.91667 \\
\hline 0.41667 & 0.58333 & 0.08333 & 0.91667 & 0.25 & 0.75 \\
\hline 0.25 & 0.41667 & 0.08333 & 0.75 & 0.58333 & 0.91667 \\
\hline 0.08333 & 0.25 & 0.41667 & 0.75 & 0.58333 & 0.91667 \\
\hline 0.25 & 0.08333 & 0.41667 & 0.58333 & 0.75 & 0.91667 \\
\hline 0.41667 & 0.58333 & 0.08333 & 0.91667 & 0.25 & 0.75 \\
\hline 0.41667 & 0.58333 & 0.08333 & 0.91667 & 0.25 & 0.75 \\
\hline & & & & & \\
\hline 0.75 & 0.25 & 0.91667 & 0.08333 & 0.58333 & 0.41667 \\
\hline 0.41667 & 0.75 & 0.25 & 0.91667 & 0.08333 & 0.58333 \\
\hline 0.58333 & 0.91667 & 0.25 & 0.75 & 0.08333 & 0.41667 \\
\hline 0.91667 & 0.75 & 0.58333 & 0.41667 & 0.25 & 0.08333 \\
\hline
\end{tabular}

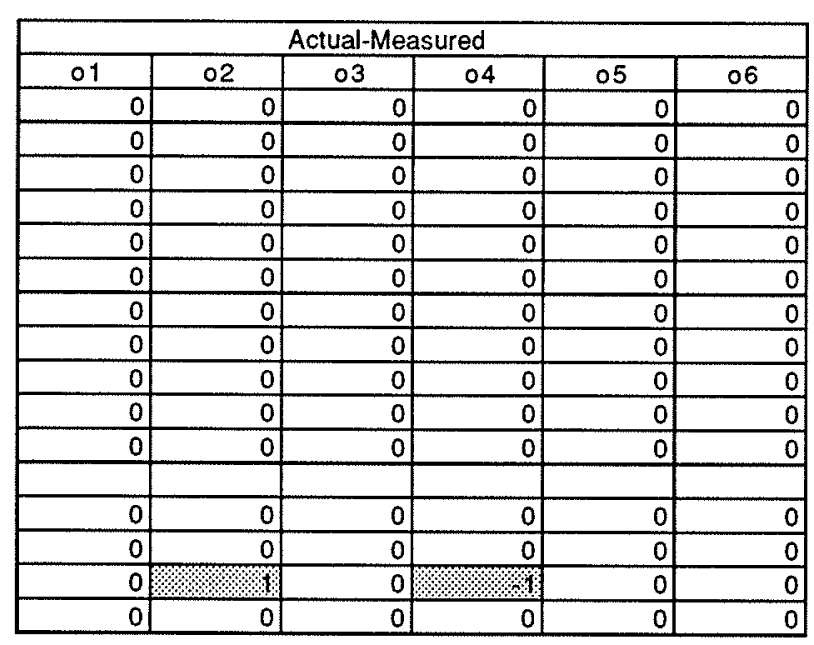


Table 4.2:

Comparison of routing performance for Sample Network for entire network and different valence values

\begin{tabular}{|c|c|c|c|c|c|}
\hline Item & $\begin{array}{c}\text { Number } \\
\text { of Nodes }\end{array}$ & $\begin{array}{c}\text { Number } \\
\text { of routes }\end{array}$ & $\begin{array}{c}\text { Number of } \\
\text { mismatches }\end{array}$ & $\begin{array}{c}\text { Error } \\
\text { (Percent) }\end{array}$ & $\begin{array}{c}\text { Accuracy } \\
\text { (Percent) }\end{array}$ \\
\hline 2 link nodes & 1 & 30 & 0 & $0 \%$ & $100 \%$ \\
\hline 3 link nodes & 3 & 135 & 0 & $0 \%$ & $100 \%$ \\
\hline 4 link nodes & 7 & 420 & 0 & $0 \%$ & $100 \%$ \\
\hline 5 link nodes & 3 & 225 & 4 & $1.78 \%$ & $98.22 \%$ \\
\hline 6 link nodes & 2 & 180 & 4 & $2.22 \%$ & $97.78 \%$ \\
\hline Full network & 16 & 990 & 8 & $0.81 \%$ & $99.19 \%$ \\
\hline
\end{tabular}

the shortest time delay between originating and destination nodes using the available links between the originating node and its adjacent nodes. In the regular case of determining the optimal route through a telecommunications network, all links are available. In the special case of determining a restoration route through a telecommunications network, one or more links are unavailable, and a choice is made between the remaining links. What is required is a method of automatically determining the best route given the availability of adjacent links.

\subsubsection{Postprocessing of the output values}

A trained neural network produces output values that represent the routing rankings of a given node in the sample network. This information now must be combined with information on availability of the links to the adjacent nodes to select the link representing the best route through the network. This route selection could be performed by postprocessing the output values with the link availability. There are several ways that this could be performed, but a neural network solution is desired considering the nature of the thesis work.

Choosing the best output from the output layer is a competitive activity. In neural networks this type of activity can be performed by using mutual inhibition, where each competing neuron provides a positive weight to itself and a negative weight to the neurons it competes against. This type of circuit 
is normally called a winner-take-all circuit, and is commonly used to select a given unit with the highest activation value. In this thesis, what is required is a "loser-take-all" circuit, where the lowest activation (smallest value) is selected. This can be achieved by using an offset activation value of 1 and subtracting the lowest activation value; in this manner the summation of 1 minus the smallest activation will produce the largest activation value.

This circuit is shown in Figure 4.1(a). In this figure the input neurons for the circuit are the outputs of the trained neural network. The input neuron is connected to the output neuron (one of the competing members of the winner-take-all circuit) by a inhibitory link with weight $=-1$. A select neuron is also connected to each output neuron by an excitory link with weight $=1$. This select neuron is used to provide link availability information: if the select neuron has an activation of 1 , the link associated with the output of the trained neural network is available; if the select neuron has an activation of 0 , the link is not available. When the link is available, the select neuron provides the offset activation value, and the sum of the activations to the competing neuron is 1 minus the input activation. When the link is not available, no offset is provided, and the sum of the activations is 0 minus the input activation. Figure 4.1(b) shows a winner-take-all circuit with all links available (all select neurons have an activation of 1 ). When the winner-takeall circuit has settled, the winning node is associated with the lowest ranking input value, provided by neuron 04 . Figure 4.1 (c) shows a winner-take-all circuit with one link not available (the unavailable link is associated with the lowest ranked input value, and the associated select neuron has an activation of 0 ). With the select neuron activation at 0 , the activation of the competing neuron is 0 minus the input activation, resulting in a negative input. The winner in this competition is the neuron associated with input value 02 as shown in Figure 4.2(d), for it now has the highest activation value of the three neurons with available links.

MacBrain provides a Group Winner activation function as one of the available activation functions; this was used to produce a winner-take-all circuit. By adding a Group Winner layer and select neurons to the trained network, the final neural network used for routing has the topology shown in Figure 4.2. 


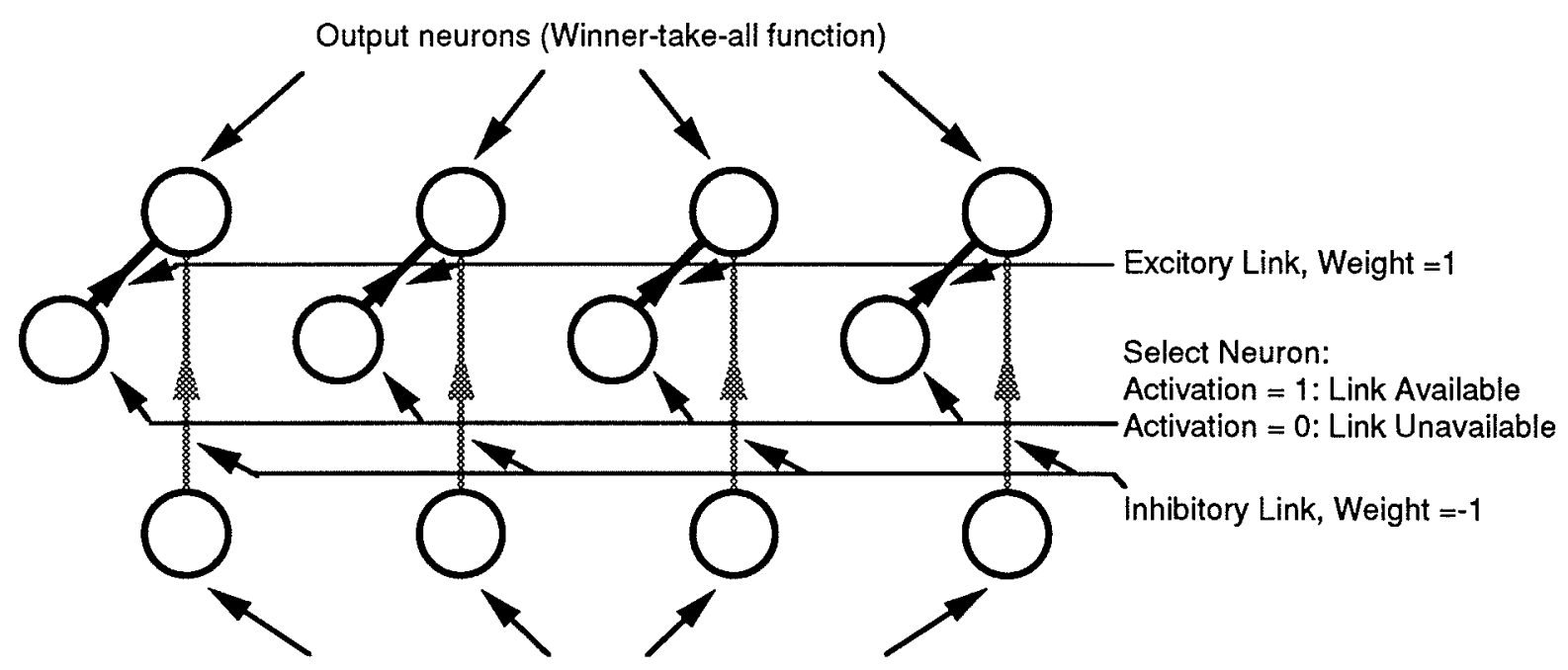

Input neurons (output Neurons from trained network)

Figure 4.1(a):

Winner-Take-All circuit

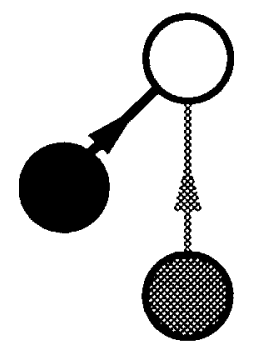

$01(4)$

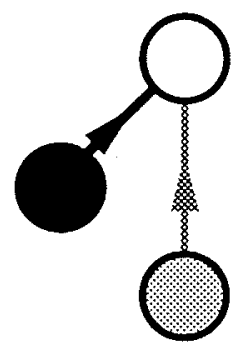

$02(2)$

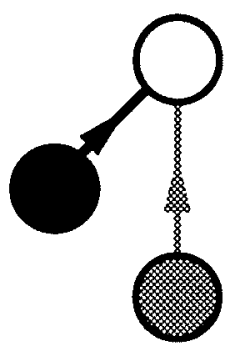

$03(3)$

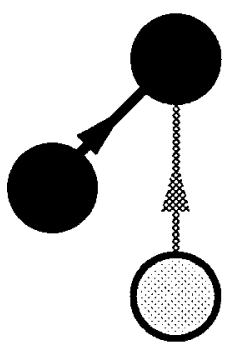

$04(1)$

Winner-take-all selects o4 output

All links available

(Activation $=1$ )

Output results from trained network. Shading indicates activation; value in brackets indicates ranking.

Figure 4.1(b):

Selection of output neuron with all links available

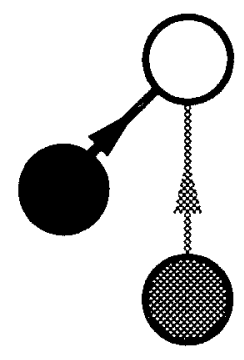

$01(4)$

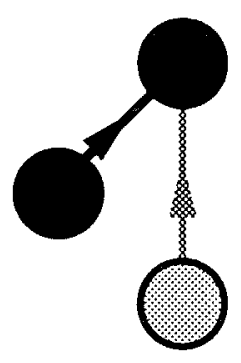

$02(2)$

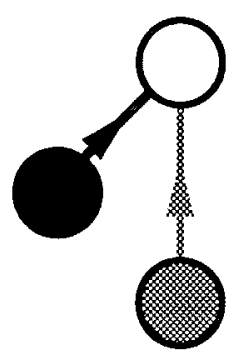

$03(3)$

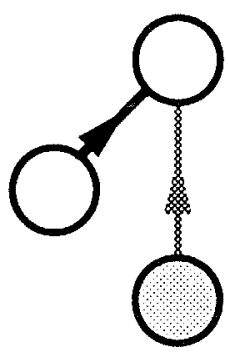

$04(1)$
Winner-take-all selects 02 output

Link related to 04 unavailable (Activation $=0$ )

Output results from trained network. Shading indicates activation; value in brackets indicates ranking.

Figure 4.1(c):

Selection of output neuron with one link unavailable 


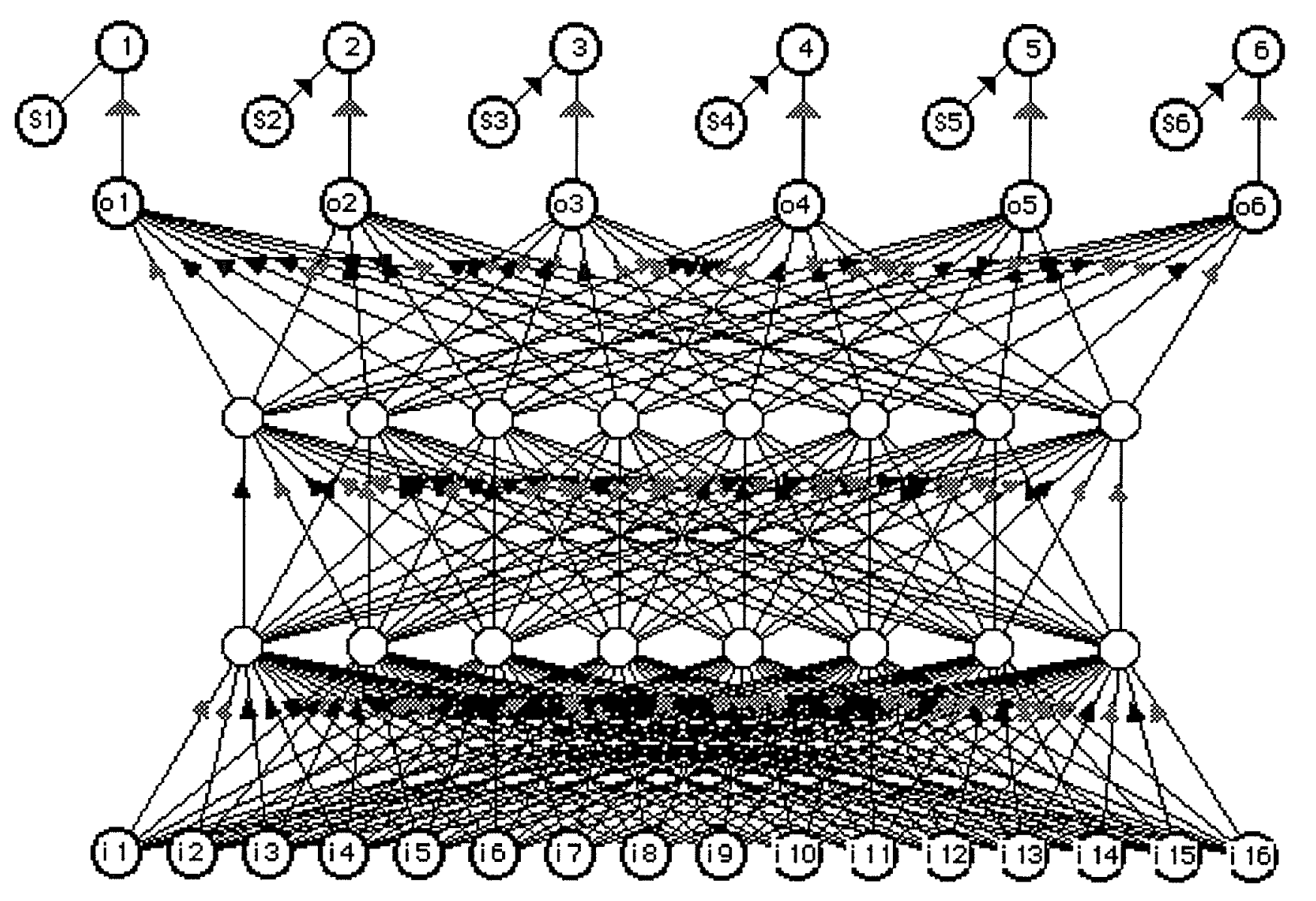

Figure 4.2:

Final neural network topology
Winner-Take-All Layer

Select neurons

(Link available/unavailable)

Output layer

(Network Information)

hidden layers,

8 neurons per hidden layer

Input layer

(Destination information) 
The inputs into the network are the destination node for which the route is to be determined, and the link availability as determined by the select nodes that provide activation to the Group Winner layer. This final neural network topology will be used in upcoming simulations.

\subsection{Simulation of a routing decision - normal network}

The first simulation of a routing decision uses the sample network in its normal configuration with all links available. In this simulation, the neural networks determine the best route based on the shortest network delay.

\subsubsection{Successful selection of optimal route}

For this simulation, Node 6 was selected as the originating node and Node 13 was selected as the destination node. Node 6 has five links to adjacent nodes, connecting it to Nodes $2,3,7,10$, and 14 . The best routes from each adjacent node to Node 13 were determined and these routes were combined with the adjacent links to create the five possible routes that can be selected by the neural network. Figure 4.3(a) shows the five routes and their associated routing values. Based on these values, the optimal route from Node 6 to Node 13 is $6-10-11-12-13$, and the link from Node 6 to Node 10 is the best choice for routing from Node 6 .

The file containing the trained neural network for Node 6 was opened, and the following neuron inputs were set: input i13 was set to 1 , all other input neurons were set to 0 , and the select neurons were all set to 1 (all links available). The input information was then cycled through the network, and the output neuron associated with 04 was activated by the winner-take-all circuit. The 04 output corresponds to the link from Node 6 to Node 10. The simulation was continued by opening the trained neural network for Node 10 , selecting the same input and select neuron information, and cycling the input values through the neural network. When the network had completed cycling 

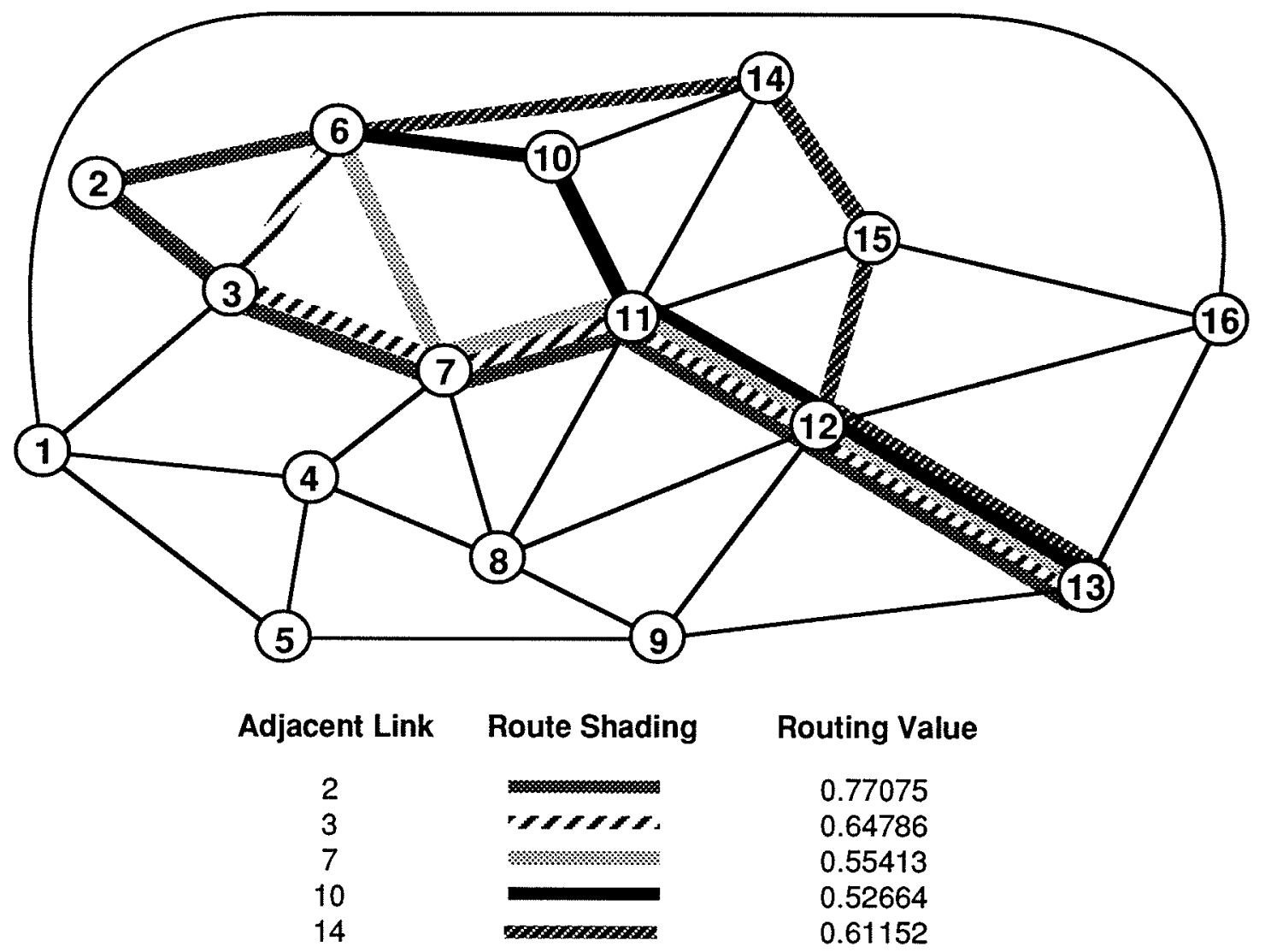

Figure 4.3(a):

Routes from Node 6 to Node 13 through available adjacent links

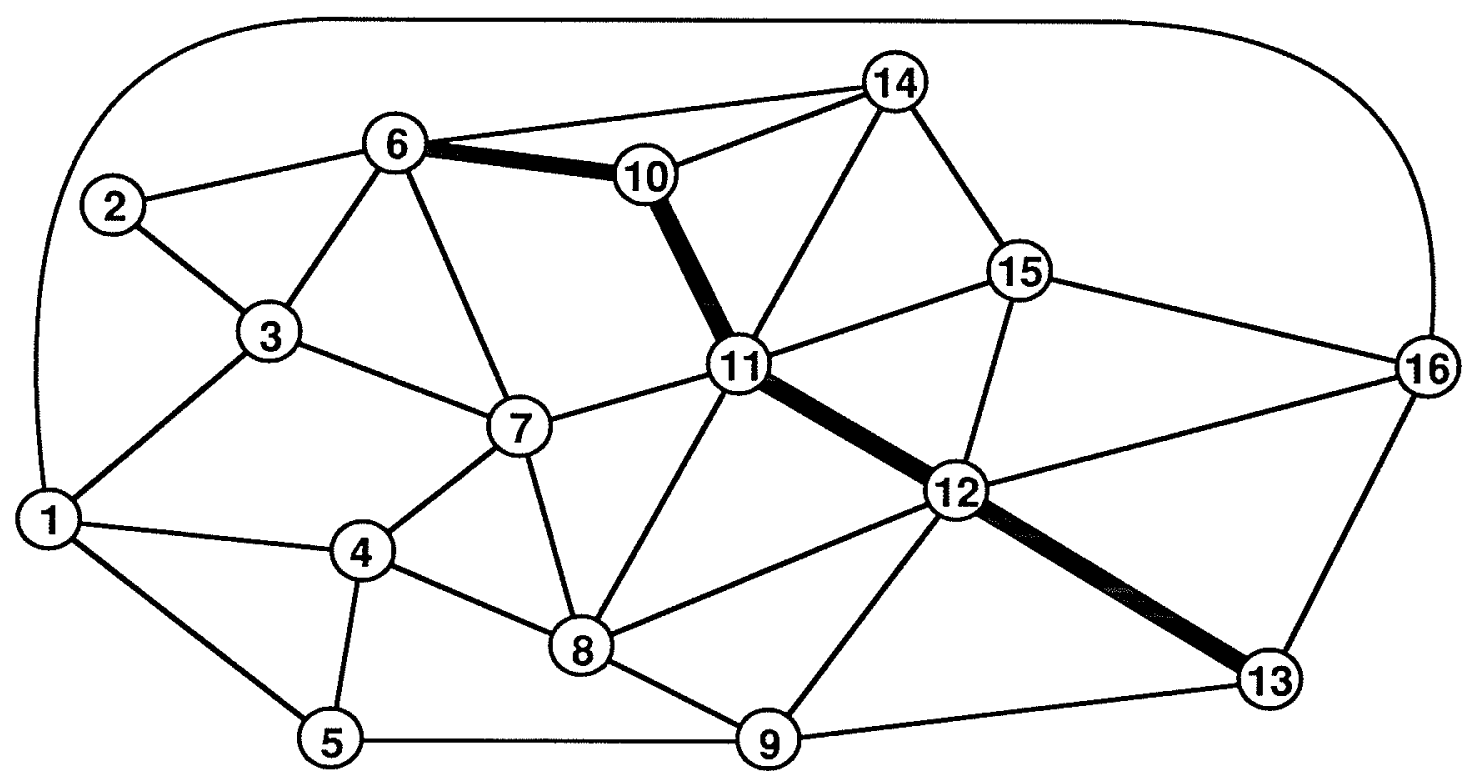

Figure 4.3(b):

Optimal route through Node 10 chosen by neural networks 
the input information, the output neuron corresponding to the link from Node 10 to 11 was selected. To complete the simulation, the process was repeated for the trained networks for Nodes 11 and 12, and the links from Node 11 to Node 12 and Node 12 to Node 13 were selected, respectively. The complete selected route was 6-10-11-12-13, the correct route identified previously. This route is shown in Figure 4.3(b)

\subsubsection{Unsuccessful selection of optimal route}

When a neural network incorrectly learned the routing sequence, a routing mismatch occurs. These routing mismatches can result in incorrect routing decisions being performed in the network.

To demonstrate the effect of a learning error producing an incorrect routing decision, the neural networks are used to determine the optimal route between Node 11 and Node 14. It can be easily determined that the optimal route is the link that directly connects Node 11 to Node 14, but in this case the neural network did not correctly learn the routing rankings. To test the effect of the learning error, the file containing the trained neural network for Node 11 was opened and the following neuron inputs were set: input i14 was set to 1, all other input neurons were set to 0 , and the select neurons were all set to 1 (all links available). The input information was then cycled through the network, and the output neuron associated with o3 was selected. The o3 output corresponds to the link from Node 11 to Node 10; the error in learning caused the network to select the link associated with output o3 instead of 05 , the link that directly connects Node 11 to Node 14 . The simulation was continued by opening the trained neural network for Node 10, selecting the same input and select neuron information, and cycling the input values through the neural network. When the network had completed cycling the input information, the output neuron corresponding to the link from Node 10 to 14 was selected. The complete route determined was 11-10-14, instead of 11-14. This result is shown in Figure 4.4(a). 


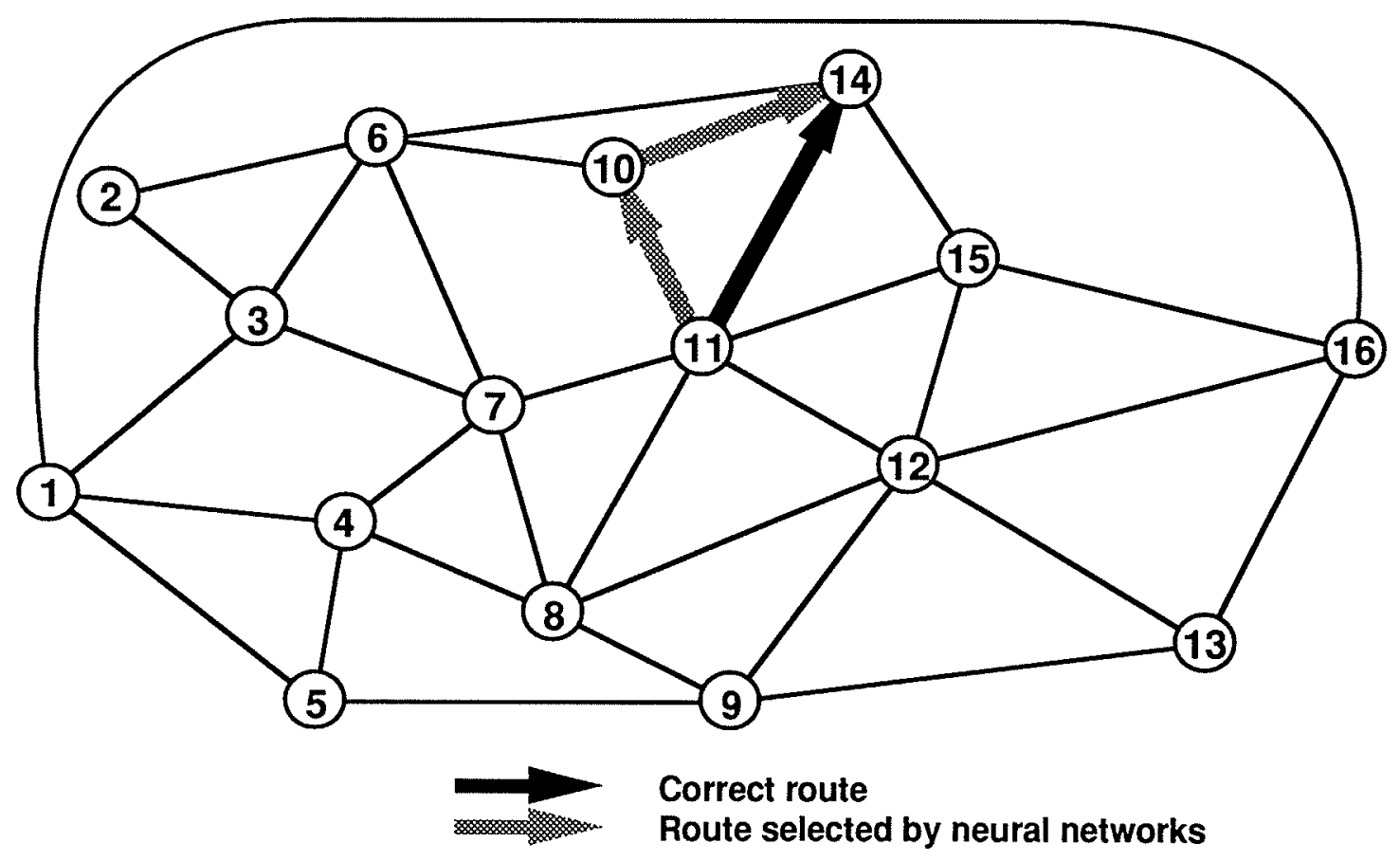

Figure 4.4(a):

Unsuccessful selection of optimal route from Node 11 to Node 14

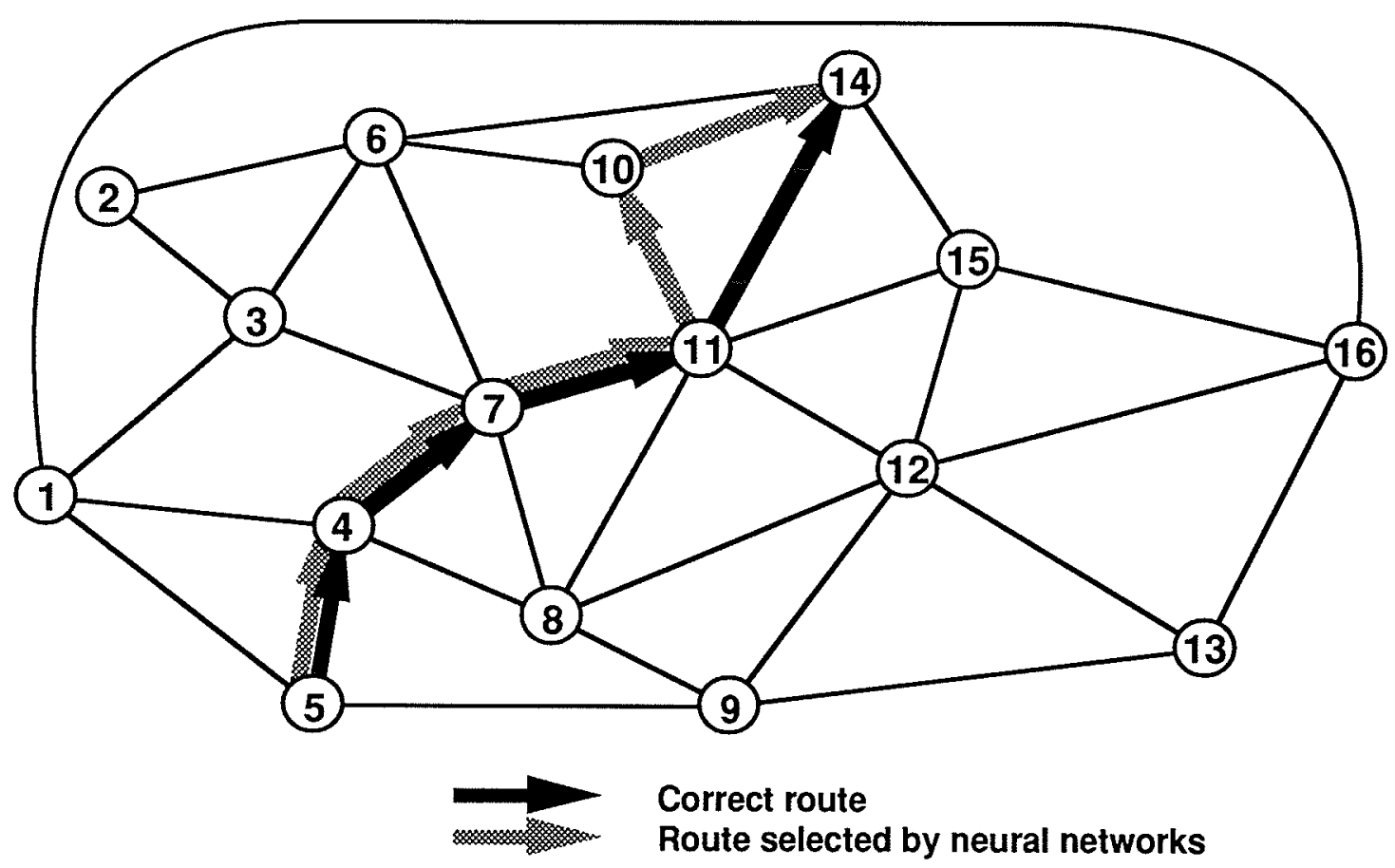

Figure 4.4(b):

Example of route impacted by unsuccessful selection in Figure 4.4(a). 
The above example shows the result of incorrect learning by the neural networks under study. It is also important to consider that this incorrect routing decision between Node 11 to Node 14 will impact other routing decisions. Any route with Node 14 as the destination node that uses the link between Node 11 and Node 14 as part of the route will have that part of the routing decision incorrectly performed. As an example, the optimal route from Node 5 to Node 14 is the route 5-4-7-11-14; but since this route includes the routing mismatch demonstrated above, the route chosen by the neural networks will be 5-4-7-11-10-14. Both routes are shown in Figure 4.4(b).

In studying the errors performed in the routing, it is important to consider that the routing error made in determining the route from Node 11 to Node 14 is not performed when the route from Node 14 to Node 11 is determined; there was no learning errors in the neural network trained for Node 14, so the correct routing decision would be performed in the opposite direction.

\subsection{Simulation of a routing decision around unavailable links}

The optimal route through the sample network with all links available was presented above. The process relied on the concept that routing decisions by the neural networks are dependent on the availability of links from each node to adjacent nodes in the network, and the optimal route consists of an originating node, a destination node, and a set of intervening links and nodes. For the following simulations, the task of the neural networks will be to determine the most optimal alternate route between two sample network nodes when a network link used by the optimal route is unavailable. These simulations are analogous to the removal of a link in the sample network, and the requirement to establish a alternate route to replace it.

Unless the neural networks are trained with new network information related to the unavailable link in sample network, the neural networks will still perform their routing decisions based on their learned information and 
adjacent link availability. As a result, any neural network for a node that is not connected to the unavailable link will perform its routing decisions as if that link is available. Only the neural networks for the nodes connected to the unavailable link will use that link information in their routing decisions.

Based on the method of routing, the determination of an alternate route can be performed by one of two methods. In the first method, an alternate route will be created from the node where the adjacent link is unavailable to the destination node. In the second method, an alternate route will be created that will replace the unavailable link only, without changing the rest of the existing optimal route.

\subsubsection{Selection of an alternate route to the destination node}

In this method, if all of the adjacent links to a node were available, the input neuron for the destination node would be set to 1, all other input neurons would be set to 0 , and all links are available. The input values would be cycled through the network, and the output routing result would be produced. If an adjacent link was unavailable, then the input neuron representing the destination would be set to 1 , all other input neurons would be set to 0 , and the link availability values set as required. When the input values are cycled to the outputs, the best adjacent link to the destination node would be selected. The node at the end of this adjacent link will then select another adjacent link that represents the optimal route to the destination node using the same method, and this is repeated until the destination node is reached. Since the new node and its alternate adjacent links are not part of the original set of the optimal intervening nodes and links, the alternative route may differ significantly from the original optimal route. In addition, since each node along the optimal route has a different set of adjacent links, the location of the unavailable link can significantly affect the resulting alternative routings.

As an example, the optimal route between Node 16 and Node 3 determined when all network links are available is $16-15-11-7-3$ as shown in Figure 4.5(a). In between Node 16 and Node 3 are four links; a removal of any one of these links 
will result in a specific neural network selecting the second choice adjacent link to determine an alternate route. Since each node in the network has a different set of adjacent links, the alternate solution for each node along the route will be different. Figure 4.5 (b) shows the alternate routes determined by the neural networks for the unavailability of different links in the network.

A study of the routing decisions above also reveals that the alternate route selected will also be dependent on the direction that the routing is performed, for the nodes on each end of the unavailable link can select different alternate links and nodes. To demonstrate this, routing decisions were performed from Node 3 to Node 16, the opposite direction to the previous routing simulation. Figure $4.6(a)$ is a duplicate of Figure $4.5(b)$, which shows the route selections made when the routing was performed from Node 16 to Node 3. Figure $4.6(\mathrm{~b})$ shows the route selections made for the same unavailable links, but in this example the routing was performed from Node 3 to Node 16 . In comparing the two figures it can be seen that for a given unavailable link the neural networks selected different alternate routes, with these routes being dependent upon the direction the routing is taking place.

\subsubsection{Selection or alternate routing around an unavailable link}

In the previous method, an alternate optimal route was created from a node with an unavailable link to the destination node. This method, while effective in establishing an alternate routing, resulted in alternative routings that were significantly different than the original optimal route. This can be useful in some forms of communications networks (such a packet networks where knowledge of the exact routing is not required) but the primary requirement in current telecommunications networks is to establish an alternate route around an unavailable link. This second routing method satisfies this requirement.

To test the routing activity of the neural networks using this method, a modified routing procedure was required. For this method, if all of the adjacent links of a node were available, the input neuron for the destination 


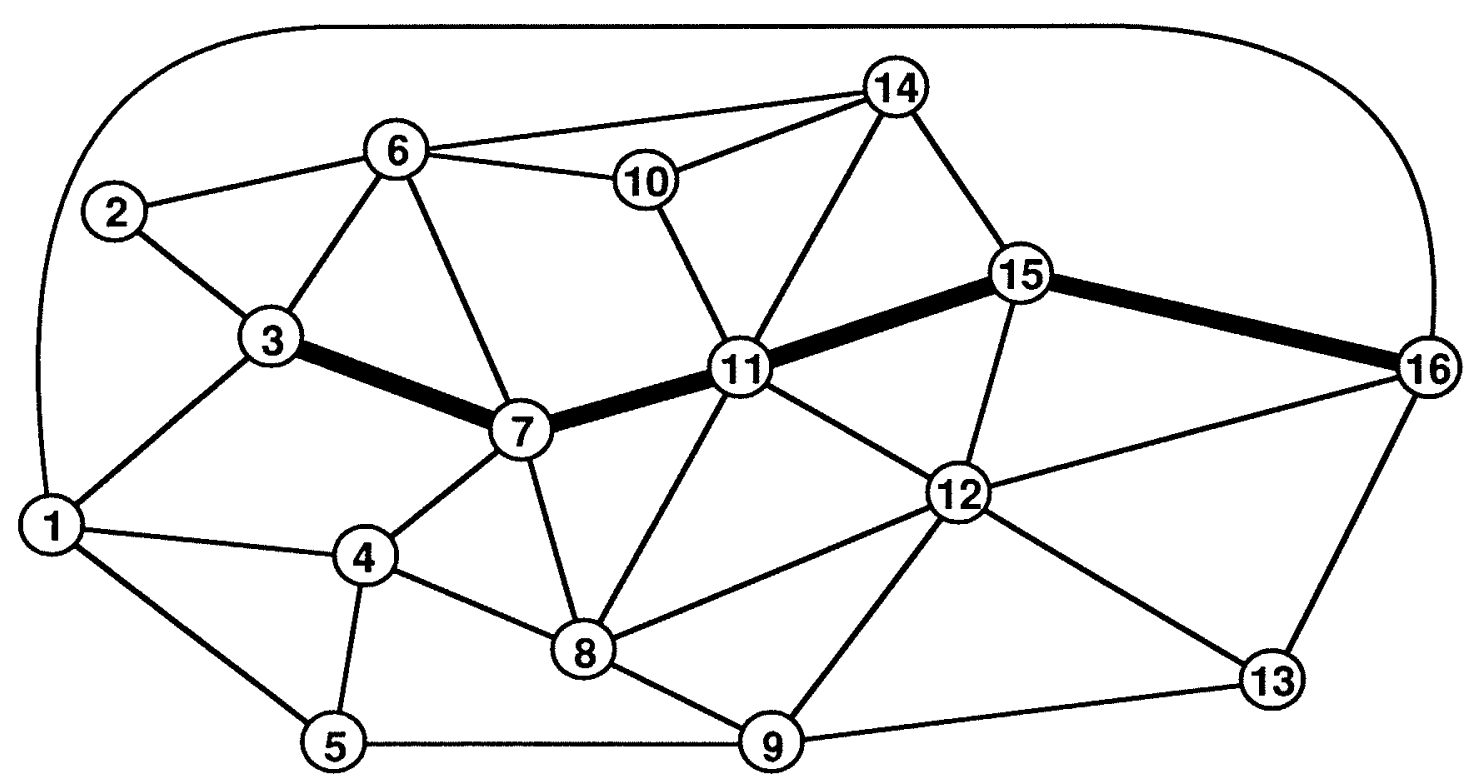

Figure 4.5(a)

Optimal Route from Node 16 to Node 3 with all links available

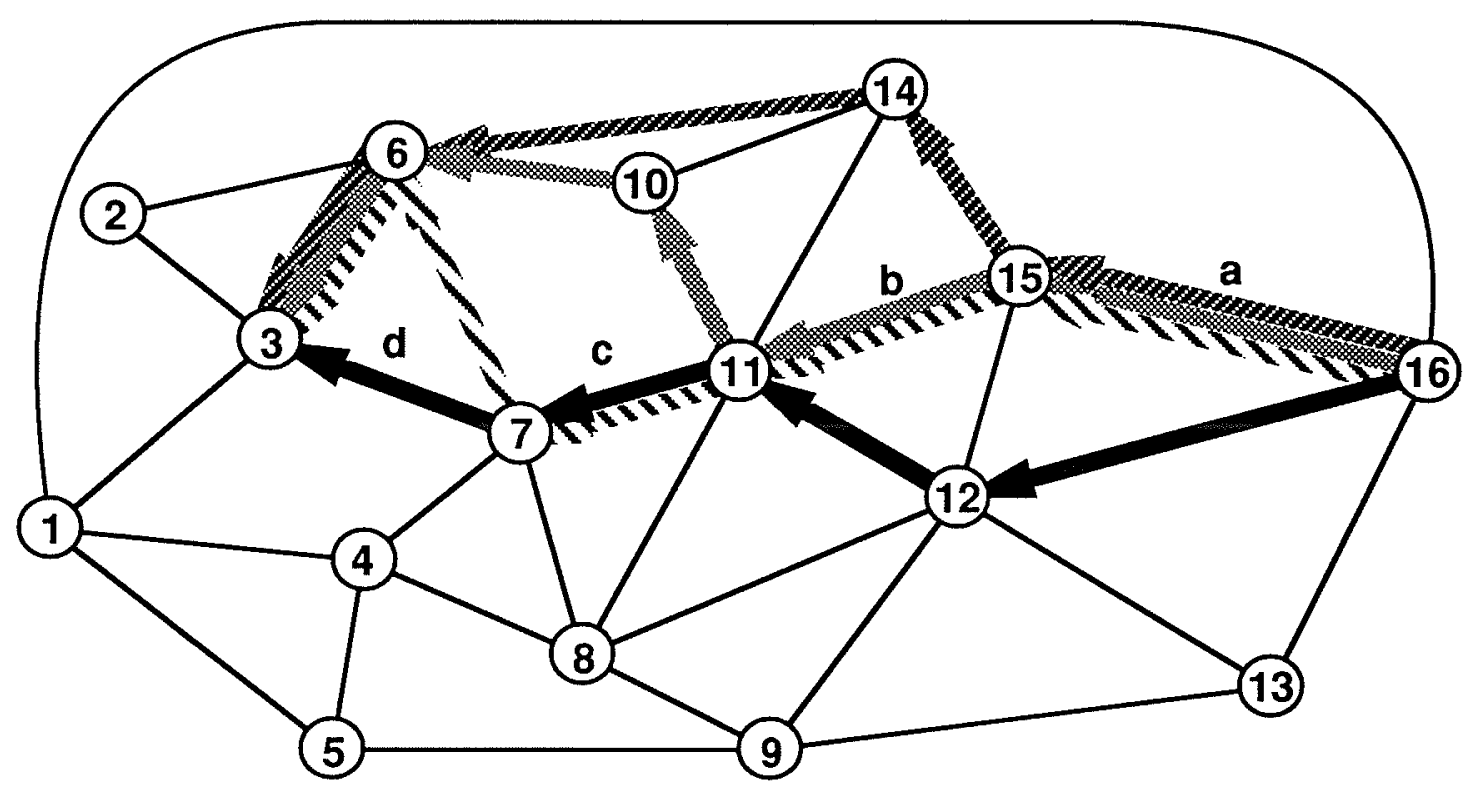

Unavailable Link Route Shading New Optimal Route Alternate routing at

$\begin{array}{llcc}a & 16-12-11-7-3 & 16 \\ \text { b } & 16-15-14-6-3 & 15 \\ c & 16-15-11-10-6-3 & 11 \\ \text { d } & 16-15-11-7-6-3 & 7\end{array}$

Figure 4.5(b)

Alternate routes determined as a function of unavailable link (Routing from Node 16 to Node 3) 


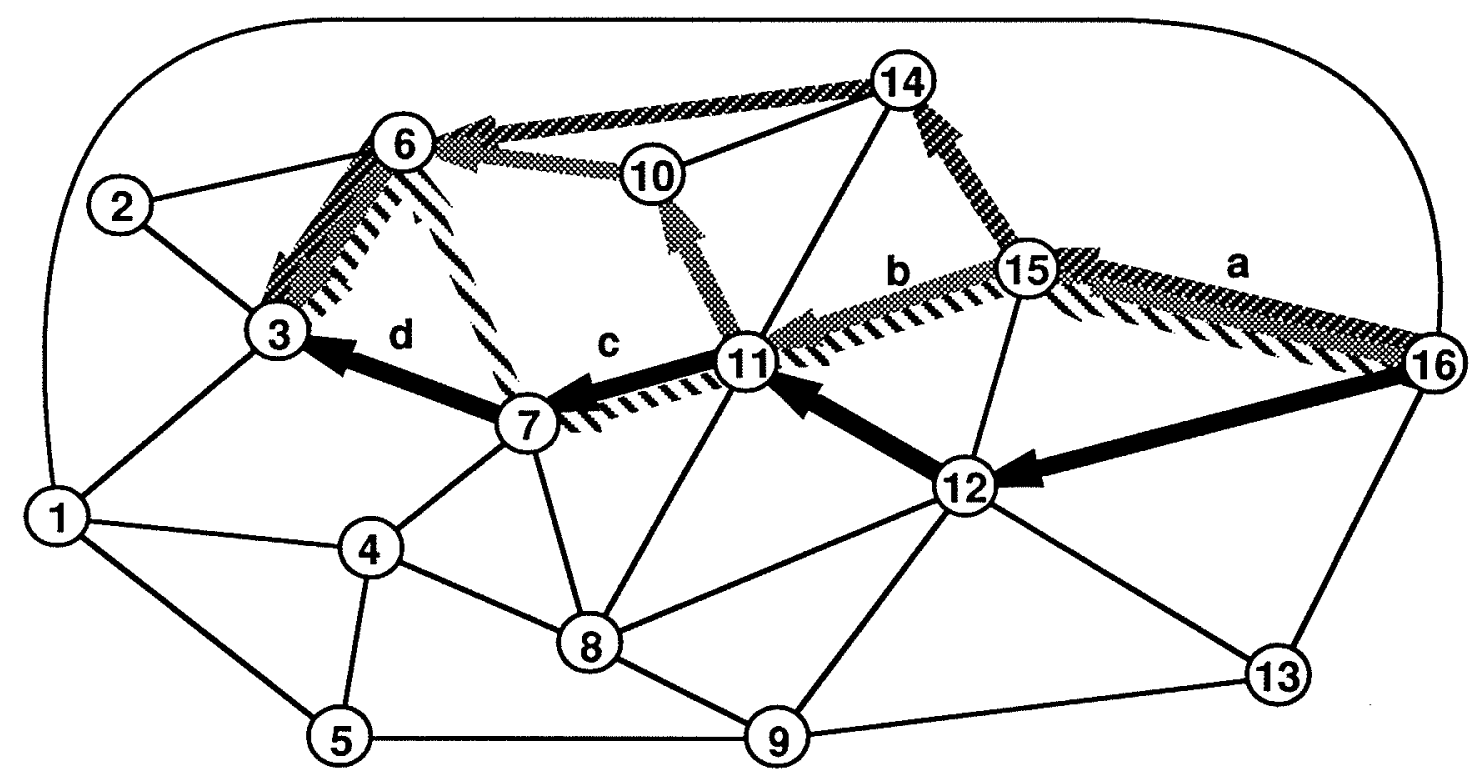

Unavailable Link Route Shading

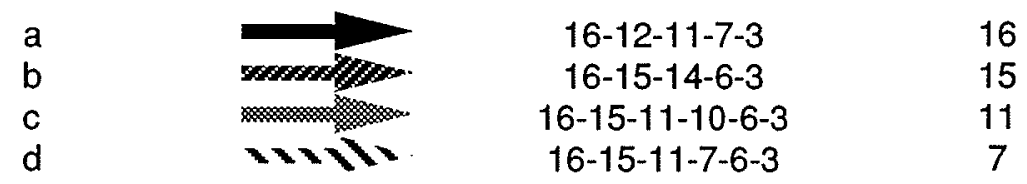

Figure 4.6(a)

Alternate routes determined as a function of unavailable link

(Routing from Node 16 to Node 3)

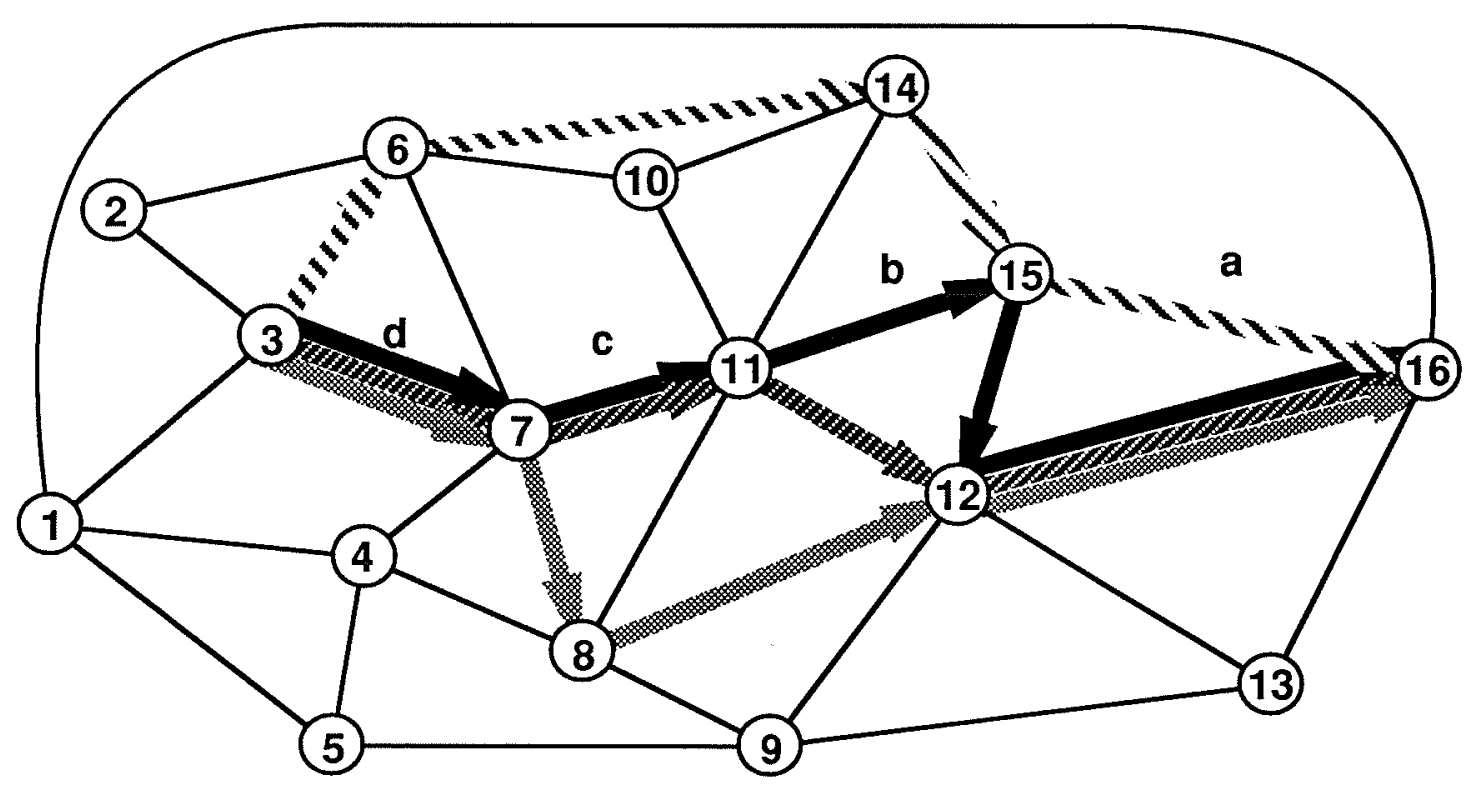

Unavailable Link Route Shading Alternate Route Alternate routing at

$\begin{array}{llcc}\text { a } & 3-7-11-15-12-16 & 15 \\ \text { b } & 3-7-11-12-16 & 11 \\ \text { c } & 3-7-8-12-16 & 7 \\ \text { d } & & 3-6-14-15-16 & 3\end{array}$

Figure $4.6(b)$

Alternate routes determined as a function of unavailable link (Routing from Node 3 to Node 16) 
node would be set to 1 , all other input neurons would be set to 0 , and all links are available. The input values would be cycled through the network, and the output routing result produced. If, however, the adjacent link used in the original optimal route was unavailable, then the input neuron for the sample network node at the other end of the unavailable adjacent link would be set to 1 , all other input neurons would be set to 0 , and the link availability values set as required. When the input values are cycled to the outputs, the best adjacent link to the node on the other end of the unavailable link would be selected. In this manner the node at the other end of the unavailable link becomes an interim destination node, and routing continues through the network until this node is reached. When the node at the other end of the unavailable link is reached, routing towards the original destination node is resumed, and the routing after the unavailable link is continued until the destination node is reached.

Using this method of routing for an unavailable link has two advantages over the original routing method: first, all nodes and all but one of the links used in the original optimal route are reused for the alternate route; and second, this form of routing produces the same results if the routing is performed in either direction. Figure 4.7 shows the results of using this routing method for the different unavailable links tested in the previous routing simulation.

\subsubsection{Unsuccessful selection of alternate route}

The above simulations demonstrate the successful determination of alternate routes around an unavailable link. In reviewing the learning of the neural networks, no unsuccessful alternate routings could be performed. The reason for this result was discussed in single network node modelling; in the case of performing alternative routing around an unavailable link, routing mismatches between rank 1 and rank 2 values have no effect on performance. Of the 4 routing mismatches that occurred in the 16 neural networks used, two were rank $1 /$ rank 2 mismatches and two were rank 5/rank 6 mismatches. The rank 5/rank 6 mismatches will also have no effect in the single link failure 


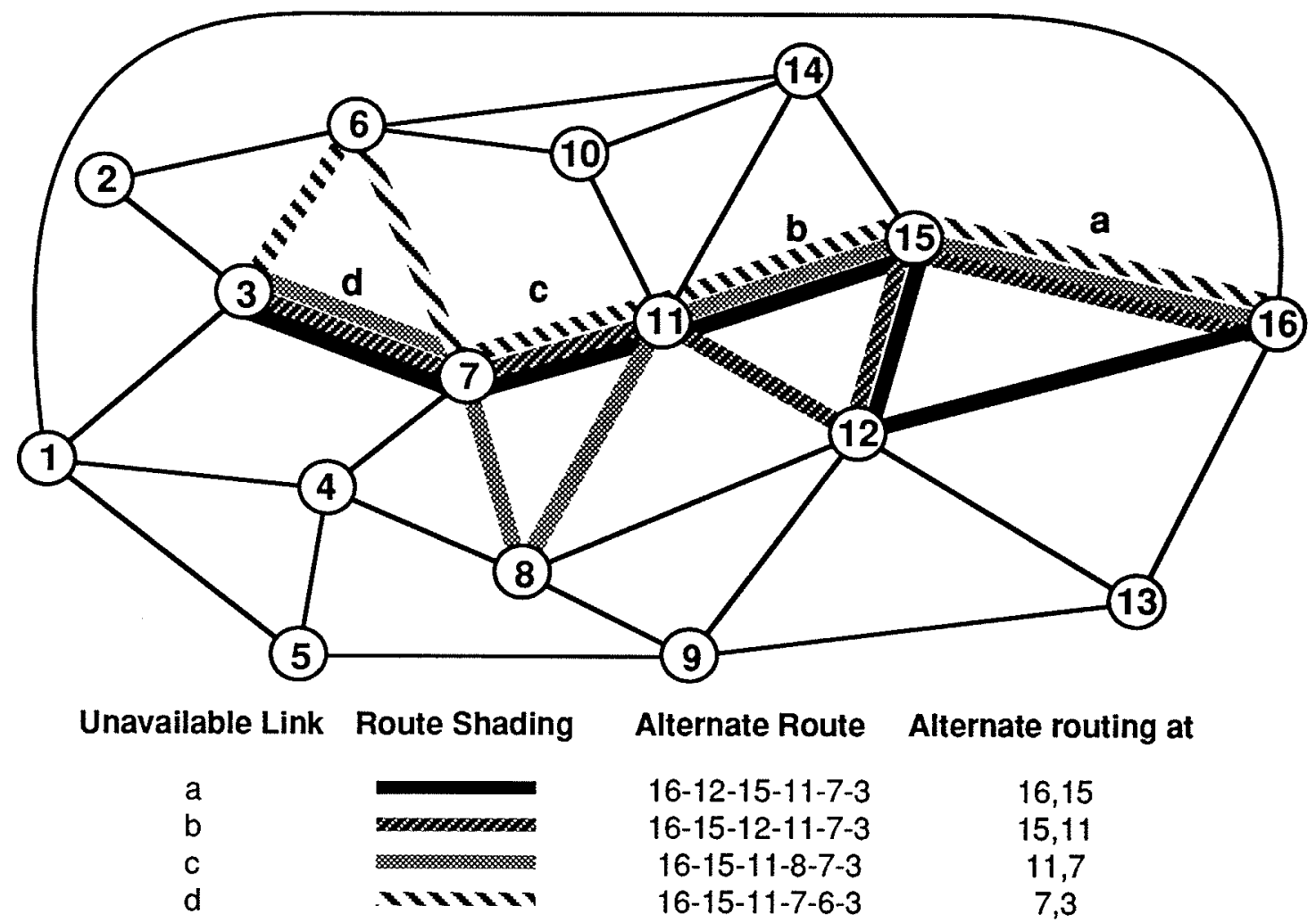

Figure 4.7:

Alternate routes around unavailable links for each unavailable link

case, for these alternative routes are too far down on the routing priority to affect this type of routing decision.

\subsection{Simulation of a routing decision - node failure}

In the previous set of simulations, routing decisions were performed for the case when a single link between two nodes is unavailable; this is an example of a link removal or failure. If a specific node is unavailable in the network, this is equivalent to all links associated with that node being made unavailable. This is an example of a node removal.

The method of performing routing decisions in the case of a node failure is similar to the link failure case; nodes in the sample network that were not directly connected to the removed node will perform routing decisions as if no 
network changes have occurred, and all nodes will still perform routing decisions based on the routing rankings of available links. But there are special considerations in the case of a node failure. These are:

- If the node that has been removed from the network is the originating node, it will not be performing any routing decisions.

- Selection of an alternate route around an unavailable link (the second form of routing utilized for link failures) cannot be performed, for this method attempts to create an alternate route to a node on the far end of the failed link; in this case that node is unavailable.

- If the node removed from the network is the destination node, no final route can be determined. It will be shown that the attempt to perform the alternate routing selection will cause the neural networks to define a route circling the removed node.

\subsubsection{Routing around an unavailable node between originating and destination nodes}

In the first method of selecting an alternate route (selecting an alternate route to the destination node), the first node along the optimal route that experiences an unavailable link selects an adjacent link that provides the best alternative route to the destination node. The node at the end of this adjacent link will then select another adjacent link that represents the optimal route to the destination node, and the process is continued until the destination node is reached. If any of the nodes along this alternate route have adjacent links that connect to the unavailable node, those links will be unavailable and not used in the route selection. As was demonstrated in the unavailable link case, the alternate routings selected are dependent on the direction that the route is determined, and that these routes can be significantly different. An example of the first method of alternate route selection around an unavailable node is shown in Figure 4.8, where an alternate route from Node 3 to Node 16 is required when Node 11 is unavailable. 

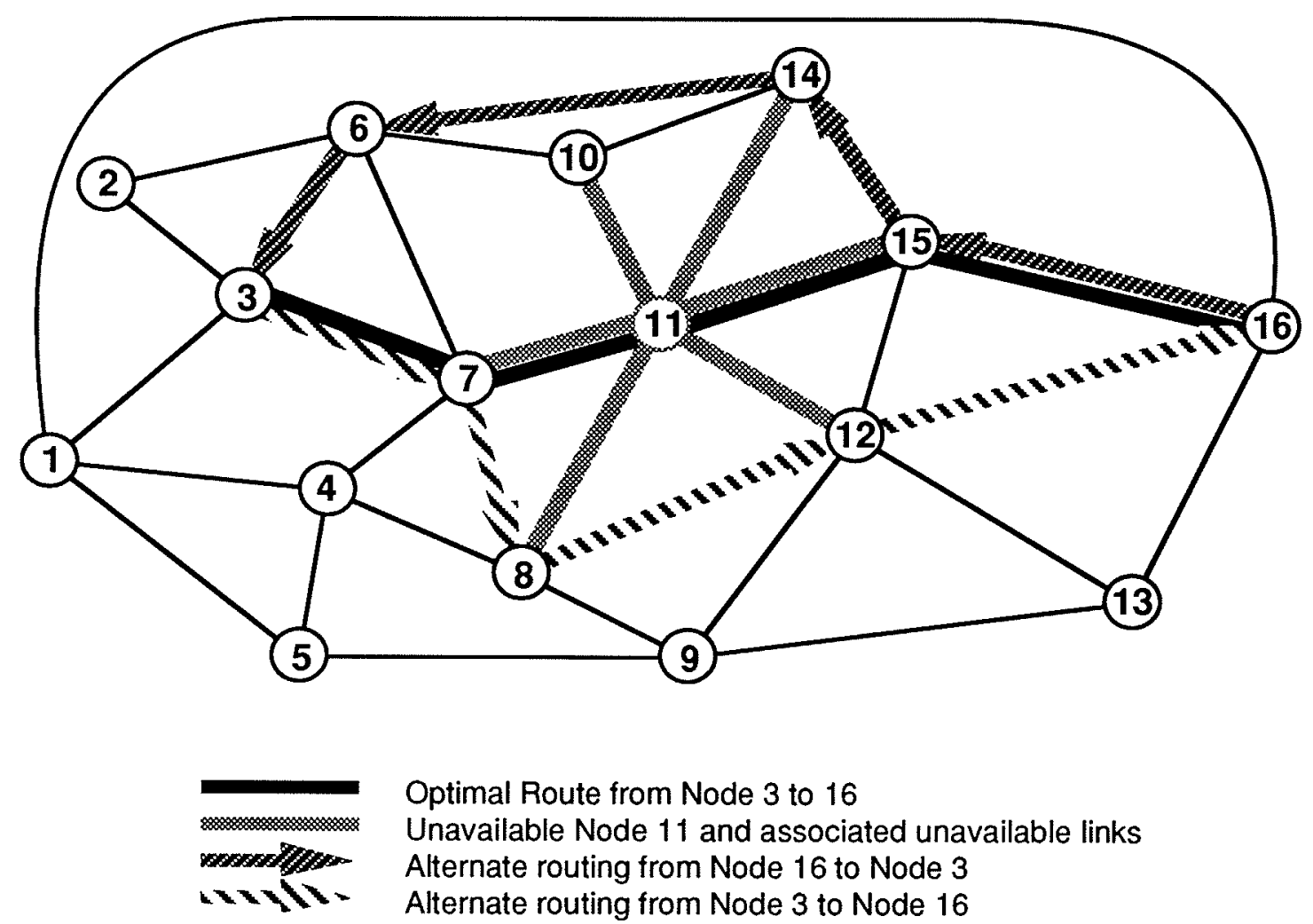

Figure 4.8:

Alternate routing around unavailable node to destination node

In the second method of selecting an alternate route (selecting an alternate route around an unavailable link), the method is the same as for an unavailable link until the point where the selection of an alternate path to the other end of the unavailable link is attempted. If another unavailable link to the node on the opposite end of the first unavailable link is encountered, then the routing decision would be changed from the node at the end of the unavailable link back to the original destination node. This change of routing destination will result in similar routing selections to those determined by the first method. In Figure 4.9(a), the second method of route selection is applied to the example of Node 11 unavailable on a route between Node 3 and Node 16. In this case the alternative routes determined were very similar in both directions, but in Figure 4.9(b) the example shows that significantly different alternate routes can determined by each direction using this method. 


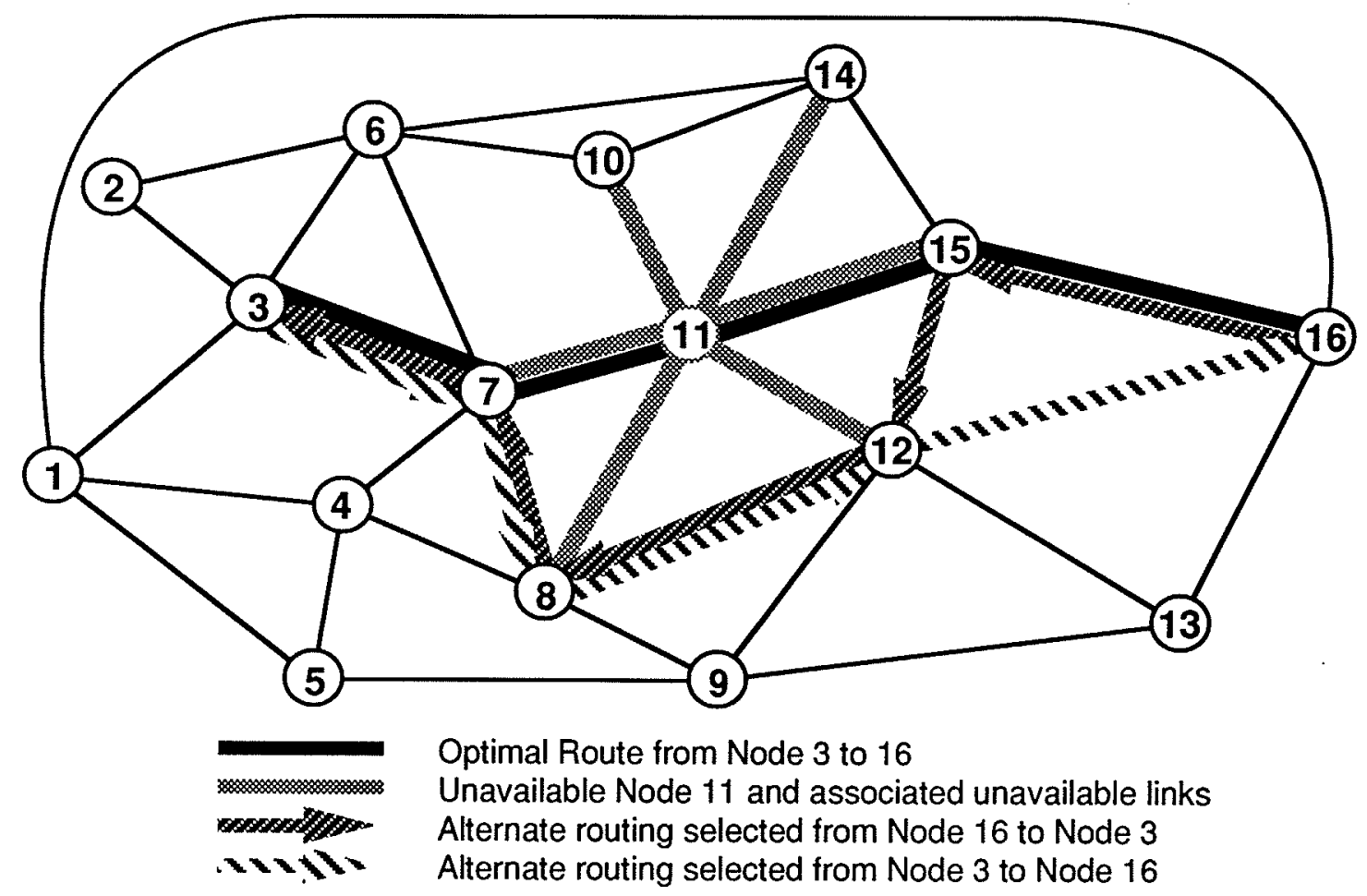

Figure 4.9(a)

Alternate routing around an unavailable node

Routing to destination node after first attempting to route around first unavailable link
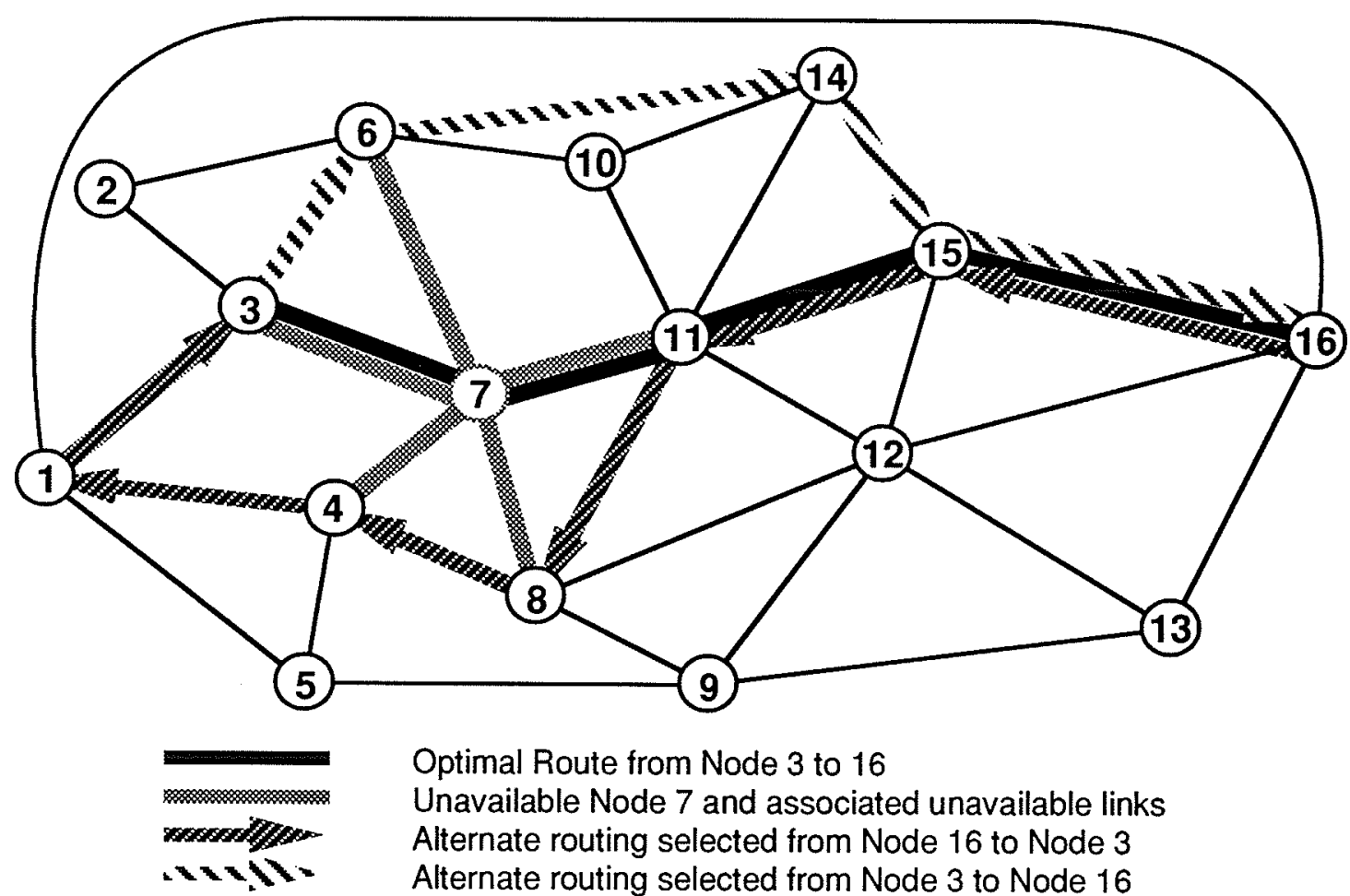

Optimal Route from Node 3 to 16

Unavailable Node 7 and associated unavailable links

Alternate routing selected from Node 16 to Node 3

Alternate routing selected from Node 3 to Node 16

Figure 4.9(b)

Second example of alternate routing around an unavailable node demonstrating significant differences between alternate routes 


\subsubsection{Routing determination when the destination node is unavailable}

If the unavailable node is the actual destination node, a complete route cannot be determined. When this condition was tested, the neural networks selected alternate routes to other nodes with unavailable links associated with the unavailable node. This function was seen in part in Figure 4.9(b), where the alternative route from Node 16 to Node 3 utilized several of the adjacent nodes with unavailable links associated with Node 7. In Figure 4.10, Node 7 is now an unavailable destination node, and the alternative route the neural networks attempted to determine starts at Node 11 and circles Node 7, linking to all other nodes with unavailable adjacent links until it returns to Node 11 . In attempting to determine the alternate route, a condition that the neural network would not select the adjacent link that connects to the previous node was used; this was based on the fact that the previous node chose the current
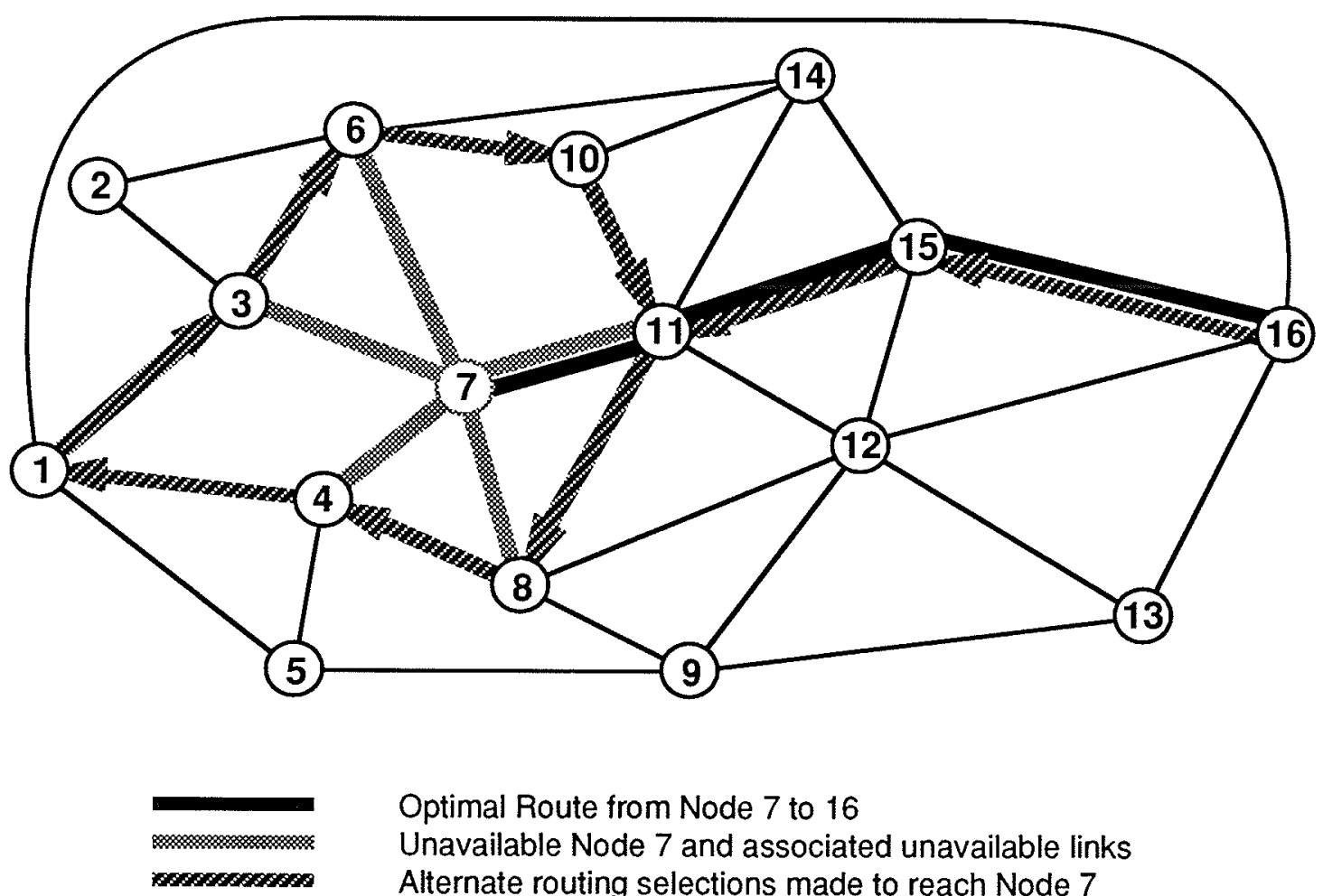

Optimal Route from Node 7 to 16 Unavailable Node 7 and associated unavailable links Alternate routing selections made to reach Node 7

Figure 4.10:

Attempt to determine alternate routing for an unavailable destination node 
node to continue with the alternate routing. If this condition was not used, then Node 4 would have chosen Node 8 in Figure 4.10 again, for the adjacent link to Node 8 is the second ranked choice from Node 4 to Node 7 after the unavailable link from Node 4 to Node 7 .

Unless a decision to stop the routing attempt is made, the process would continue indefinitely. Like the earlier situation where the routing destination was changed from the node at the other end of the unavailable link to the destination node when a second unavailable link was encountered, a decision external to the neural networks making the routing decision is required. A decision to terminate the routing attempt could be made after encountering a number of unavailable links, or it could be made when the alternate route encounters a node that had previously attempted to determine an alternate route; in the case of Figure 4.10, this would occur when Node 11 is reached.

\subsubsection{Unsuccessful selection of alternate route}

As with the simulations for unavailable links, the above simulations demonstrate the successful determination of alternate routes around an unavailable node when it is not an originating or destination node.

A review of the learning of the neural networks found that an alternate routing could always be determined, but since the training information is based on the availability of the adjacent links only, the alternate route may not be the best alternative available, for other unavailable links not directly connected to each node affect the final route. Apart from this condition, alternative routings around an unavailable node are equivalent to routing around multiple unavailable links. As in the routing determinations for unavailable links, routing mismatches between rank 1 and rank 2 values have no effect on performing routing decisions. Of the 4 routing mismatches that occurred in the 16 neural networks used, two were rank $1 /$ rank 2 mismatches and two were rank 5/rank 6 mismatches. The rank 5/rank 6 mismatches will have no effect in the unavailable node cases, for these alternative routes are too far down on the routing priority to affect almost all alternative routing 
decisions.

\subsection{Simulation of generalized routing decisions}

The final set of tests performed with the neural networks tested the ability to perform a generalization function. In this set of tests, the values of more than one input neuron were set to non-zero values to observe the neural network activity. Setting multiple input neurons to non-zero values is analogous to having the network determine which output neuron represents the best routing choice for a set of destination nodes.

Six test examples were used to investigate generalization, with the results of these tests provided in Tables 4.3 to 4.8 . In each test 2 or 3 input neurons of specific neural networks were set to non-zero values and cycled to propagate the information to their outputs. The output values of the neural network were collected and rank values calculated; these rankings were then compared with the output node rankings of the neural network for each destination node, and the ranking of the output nodes by the sum of the actual routing values (network delays) for the set of desired destination nodes. Figures 4.11(a) and (b) depict two of the generalization tests, their optimal routes for the respective destination nodes, and the optimal links based on network delays. Figure 4.11(a) depicts the condition where both destination nodes have the same optimal route, resulting in a simple decision. Figure 4.11(b) depicts the condition where the destination nodes have different optimal routes, and the optimal link derived from network delay is used by one of the routes. This is a more complex decision, and in this test the neural network made different link selections based on the method of input activation. The different methods of input activation are discussed below.

The tests were initially performed with activation of the input neurons representing the set of destination nodes set to 1 , and all other inputs neurons set to 0 . When this method of activation was used, it was found that for some tests the activation values of the output neurons approached or reached the 


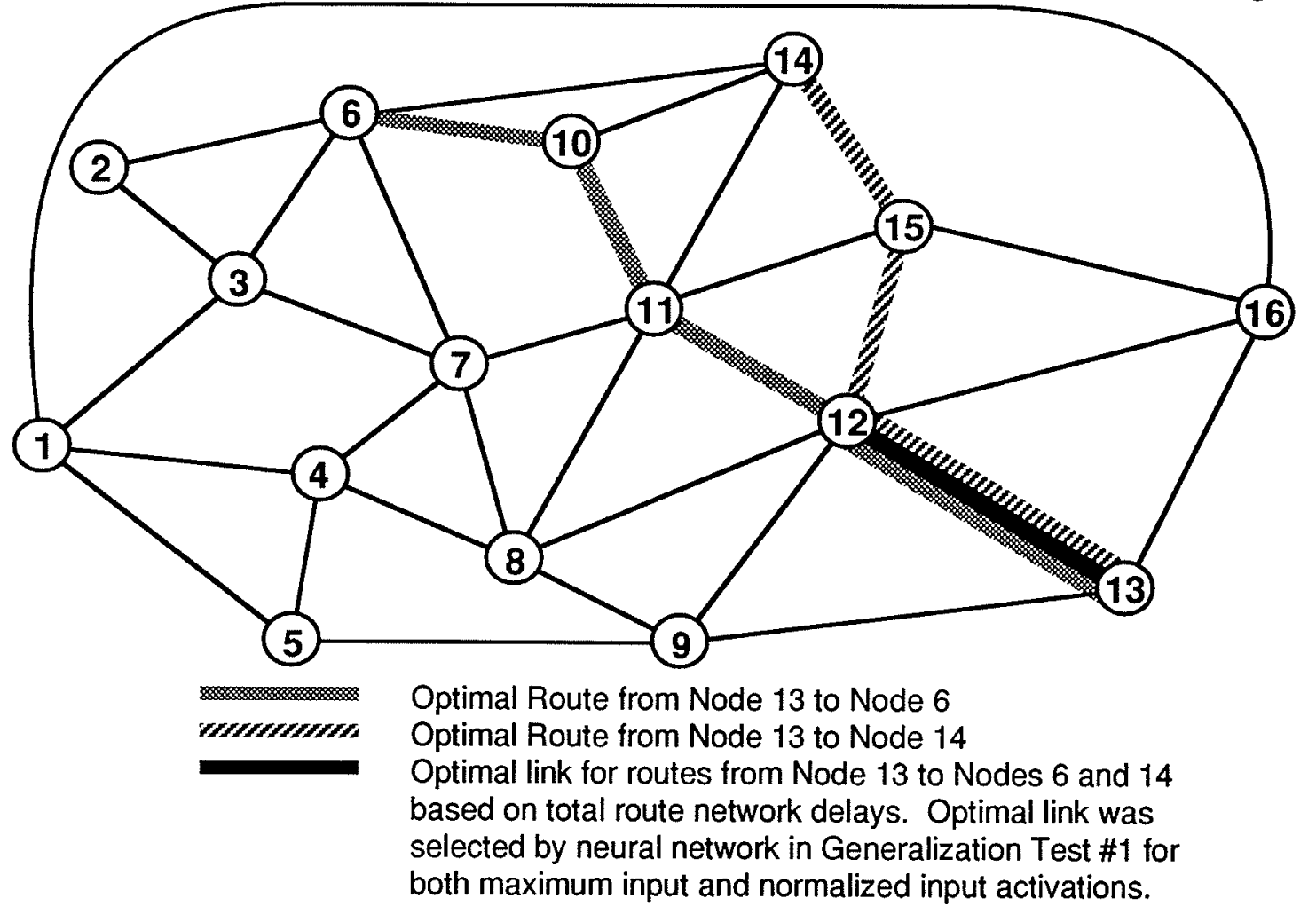

Figure 4.11(a)

Generalized routing example where 2 nodes have the same first choice link

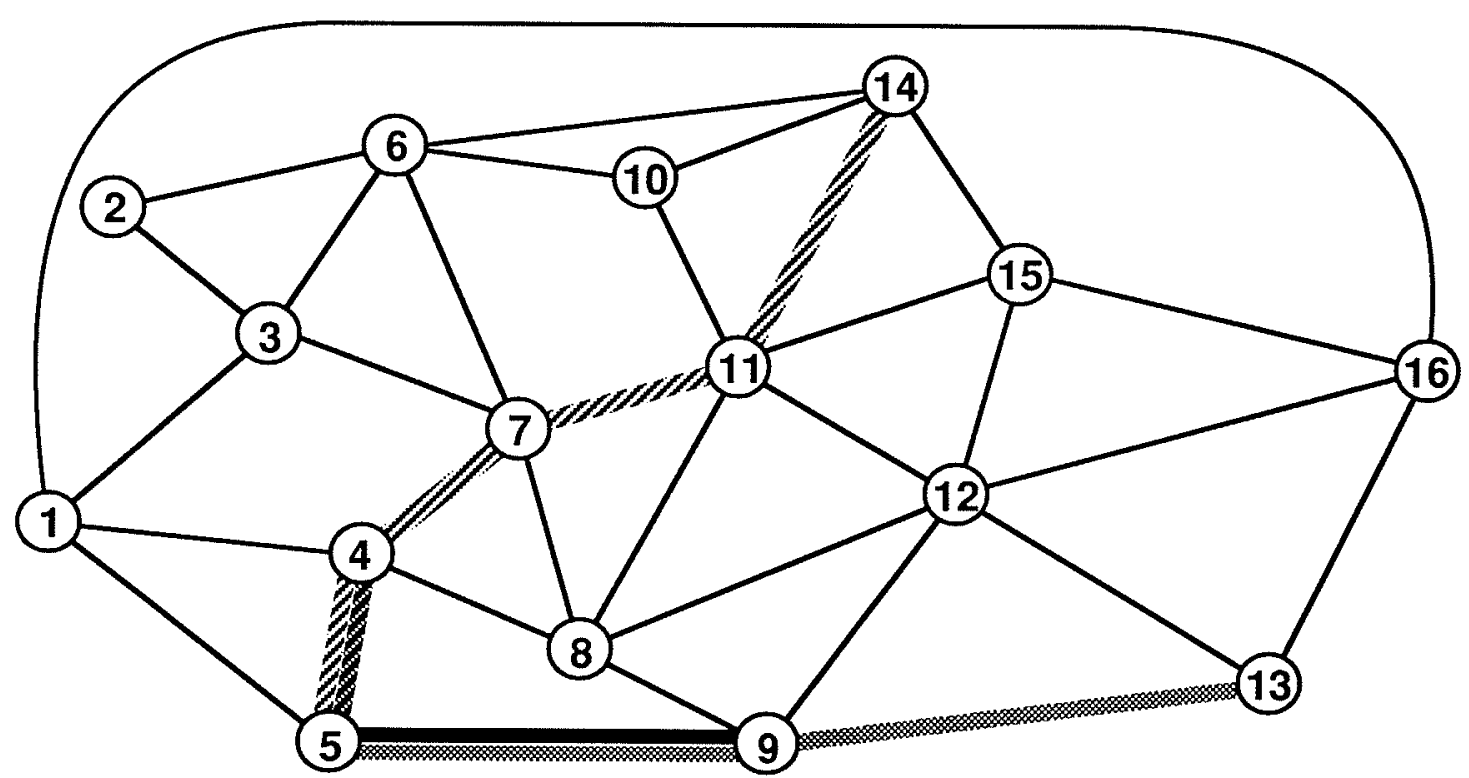

Optimal Route from Node 5 to Node 13

Oprimal Route from Node 5 to Node 14

Optimal link for routes from Node 5 to Nodes 13 and 14

based on total route network delays. Optimal link was

selected by neural network in Generalization Test \#2 with

maximum input activation.

$10000000000 \quad$ Link selected in Generalization Test \#2 with normalized input activation.

Figure 4.11(b)

Genralized routing example where 2 nodes have different first choice links 
maximum activation value of 1 . This saturation of the output values occurred because the neural networks were initially trained with training vectors having only a single input neuron set to 1 . To reduce the occurrence of saturation, a variation of the input activation method was also tested; in this second method, the activation values for the set of destination nodes were set to the reciprocal of the number of destination nodes. This form of normalized activation resulted in the total of the input activation values being equal to 1 . In the case of 2 active input neurons the activation was set at 0.5 , and for three active input neurons the activation was set at 0.33 .

\subsubsection{Generalization test results}

Test \#1 is an example of performing a decision from a 3 output originating node (Node 13) to two destinations (Nodes 6 and 14) that have the same first choice route. The results of the test are provided in Table 4.3, with output values of rank 1 shaded for comparison purposes. In this case, the optimal routes from Node 13 to Nodes 6 and 14 both utilize the link from Node 13 to Node 12. For both input activation methods the neural network selected the correct route, but the rankings of the other routes changed with the different methods of activation. When the input activation was normalized, the resulting output activations were smaller that when both input neurons were set to an activation of 1 ; this observation was also observed in the rest of the generalization tests.

Test \#2 is an example of performing a decision from a 3 output originating node (Node 5) to two destination nodes (Nodes 13 and 14) with different first choice routes. The results of the test are provided in Table 4.4, with output values of rank 1 shaded for comparison purposes. When the input activations were set to 1 , the neural network generated the routing ranking based on total network delay and the output ranking to Node 13, and selected the optimal route for both destination nodes. When the input activations were normalized, the neural network selected the output rankings of Node 14 , and selected the 2 nd choice route for the two destination nodes. 
Test \#3 is an example of performing a decision from a 5 output originating node (Node 8) to two destination nodes (Nodes 6 and 12) with significantly different output rankings. The results of the test are provided in Table 4.5, with output values of rank 1 shaded for comparison purposes. When the rankings of the outputs based on the total delay were calculated, these rankings were found to be different from the output rankings of the two destination nodes. When this test was performed, both methods of activation produced the correct routing selection, though the routing rankings of the outputs with the normalized activation were closer to the total delay ranking values than the output rankings produced when the input activations were set at 1. This test also showed that when input activations were set at 1, 4 of the 5 outputs neurons reached activation values of greater than 0.98 ; in this case, setting all active inputs to 1 resulted in near saturation of output values.

Test \#4 is an example of performing a decision from a 4 output originating node (Node 16) to two destination nodes (Nodes 4 and 6) with similar output rankings. The results of the test are provided in Table 4.6, with output values of rank 1 shaded for comparison purposes. When the input activations were set to 1 , the output neurons reached maximum activation and no ranking results could be determined. When the activation values were normalized, the output neurons produced almost no activation value, the opposite result of the previous test. When the output values for the normalized activation were ranked, the network selected the correct routing choice for Node 4 , but the incorrect routing choice for both nodes. The reason for these small activation values is not known, but observation of the activations of the various layers in the neural network showed high activation values in the first hidden layer and low activation values in higher layers; the combination of activation values and connection weights may have resulted in a cancellation of inputs in higher layers of the neural network.

Test \#5 is an example of performing a decision from a 4 output originating node (Node 4) to three destination nodes (Nodes 10,12, and 16) with similar output rankings. The results of the test are provided in Table 4.7, with output values of rank 1 shaded for comparison purposes. When both activation 
Table 4.3:

Test \#1: Route selection from Node 13 to Nodes 6 and 14

\begin{tabular}{|c|c|c|c|}
\hline Output Node & 01 & 02 & 03 \\
\hline Output Rankings: Node 13 to Node 6 & 2 & 曼 & 3 \\
\hline Output Rankings: Node 13 to Node 14 & 3 & 㷊 & 2 \\
\hline Total Delay for routes to Nodes 6 and 14 & 1.1881 & 0.9312 & 1.1459 \\
\hline Output Rankings by Total Delay & 3 & 1/. & 2 \\
\hline Output Node & 01 & 02 & 03 \\
\hline Output Values: $i 6=i 14=1$ & 0.9738 & 0.2234 & 0.7922 \\
\hline Output Rankings: $i 6=i 14=1$ & 3 & tis & 2 \\
\hline Output Node & 01 & 02 & 03 \\
\hline Output Values: $16=i 14=0.5$ & 0.49 & 0.0933 & 0.5057 \\
\hline Output Rankings: $i 6=i 14=0.5$ & 2 & tr. & 3 \\
\hline
\end{tabular}

Table 4.4:

Test \#2: Route selection from Node 5 to Nodes 13 and 14

\begin{tabular}{|l|c|c|c|}
\hline Output Node & 01 & 02 & 03 \\
\hline Output Rankings: Node 5 to Node 13 & 3 & 2 & $.1 \%$ \\
\hline Output Rankings: Node 5 to Node 14 & 3 & 1 & 2 \\
\hline Total Delay for routes to Nodes 6 and 14 & 1.3853 & 1.0253 & 0.9988 \\
\hline Output Rankings by Total Delay & 3 & 2 &. \\
\hline
\end{tabular}

\begin{tabular}{|l|c|c|c|}
\hline Output Node & 01 & 02 & 03 \\
\hline Output Values: $i 13=i 14=1$ & 0.8898 & 0.349 & 0.3359 \\
\hline Output Rankings: $i 13=i 14=1$ & 3 & 2 & $\%$ \\
\hline
\end{tabular}

\begin{tabular}{|l|c|c|c|}
\hline Output Node & 01 & 02 & 03 \\
\hline Output Values: $113=i 14=0.5$ & 0.7226 & 0.185 & 0.3161 \\
\hline Output Rankings: $i 13=i 14=0.5$ & 3 & & 2 \\
\hline
\end{tabular}

Table 4.5:

Test \#3: Route selection from Node 8 to Nodes 6 and 12

\begin{tabular}{|c|c|c|c|c|c|}
\hline Output Node & 01 & 02 & o3 & 04 & 05 \\
\hline Output Rankings: Node 8 to Node 6 & 2 & 策 & 5 & 3 & 4 \\
\hline Output Rankings: Node 8 to Node 12 & 5 & 4 & 2 & 3 & \% \\
\hline Total Delay for routes to Nodes 6 and 12 & 0.8448 & 0.6379 & 0.8798 & 0.6806 & 0.7436 \\
\hline Output Rankings by Total Delay & 4 & $\%$ & 5 & 2 & 3 \\
\hline
\end{tabular}

\begin{tabular}{|c|c|c|c|c|c|}
\hline Output Node & 01 & 02 & 03 & 04 & 05 \\
\hline Output Values: $i 6=i 12=1$ & 0.9889 & 0.5687 & 1 & 0.9993 & 0.9986 \\
\hline Output Rankings: i6 $=i 12=1$ & 3 & $1 \%$ & 5 & 4 & 2 \\
\hline
\end{tabular}

\begin{tabular}{|l|c|c|c|c|c|}
\hline Output Node & 01 & 02 & 03 & 04 & 05 \\
\hline Output Values: $i 6=i 12=0.5$ & 0.2017 & 0.0837 & 0.4972 & 0.1584 & 0.2503 \\
\hline Output Rankings: i6 $=i 12=0.5$ & 3 & .81 & 5 & 2 & 4 \\
\hline
\end{tabular}


Table 4.6:

Test \#4: Route selection from Node 16 to Nodes 4 and 6

\begin{tabular}{|c|c|c|c|c|}
\hline Output Node & 01 & 02 & 03 & 04 \\
\hline Output Rankings: Node 16 to Node 4 & 4 & Y. & 3 & 2 \\
\hline Output Rankings: Node 16 to Node 6 & 4 & 2 & 3 & 策 \\
\hline Total Delay for routes to Nodes 4 and 6 & 1.5835 & 1.0923 & 1.2915 & 1.0714 \\
\hline Output Rankings by Total Delay & 4 & 2 & 3 & 8. \\
\hline Output Node & 01 & 02 & 03 & 04 \\
\hline Output Values: $i 4=i 6=1$ & 1.0000 & 1.0000 & 1.0000 & 1.0000 \\
\hline Output Rankings: $i 4=i 6=1$ & 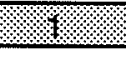 & 17 & 17 & 8 \\
\hline Output Node & 01 & 02 & 03 & 04 \\
\hline Output Values: $i 4=i 6=0.5$ & 0.0015 & 0.0012 & 0.0014 & 0.0012 \\
\hline Output Rankings: $i 4=i 6=0.5$ & 4 & 1 & 3 & 2 \\
\hline
\end{tabular}

Table 4.7:

Test \#5: Route selection from Node 4 to Nodes 10,12 and 16

\begin{tabular}{|l|c|c|c|c|}
\hline Output Node & 01 & 02 & 03 & 04 \\
\hline Output Rankings: Node 4 to Node 10 & 3 & 4 & & 2 \\
\hline Output Rankings: Node 4 to Node 12 & 4 & 3 & 2 & 2 \\
\hline Output Rankings: Node 4 to Node 16 & 4 & 3 & 2 & . \\
\hline Total Delay for routes to Nodes 10,12,16 & 1.9216 & 1.7449 & 1.2397 & 1.2251 \\
\hline Output Rankings by Total Delay & 4 & 3 & 2 & . \\
\hline
\end{tabular}

\begin{tabular}{|l|c|c|c|c|}
\hline Output Node & 01 & 02 & 03 & 04 \\
\hline Output Values: $i 10=i 12=i 16=1$ & 0.9999 & 0.9762 & 0.9406 & 0.0919 \\
\hline Output Rankings: $i 10=i 12=i 16=1$ & 4 & 3 & 2 & $\% .8 . \%$ \\
\hline
\end{tabular}

\begin{tabular}{|l|c|c|c|c|}
\hline Output Node & 01 & 02 & 03 & 04 \\
\hline Output Values: i10=i12=i16=0.33 & 0.7613 & 0.6026 & 0.2253 & 0.1884 \\
\hline Output Rankings: $i 10=i 12=\mathrm{i} 16=0.33$ & 4 & 3 & 2 & $.8 .1 \%$. \\
\hline
\end{tabular}

Table 4.8:

Test \#6: Route selection from Node 3 to Nodes 9,14 and 15

\begin{tabular}{|c|c|c|c|c|}
\hline Output Node & 01 & 02 & o3 & 04 \\
\hline Output Rankings: Node 3 to Node 9 & 3 & 4 & 2 & tis \\
\hline Output Rankings: Node 3 to Node 14 & 4 & 3 & 留 & 2 \\
\hline Output Rankings: Node 3 to Node 15 & 4 & 3 & 2 & 1 \\
\hline Total Delay for routes to Nodes $9,14,15$ & 1.8007 & 1.6461 & 1.2774 & 1.1612 \\
\hline Output Rankings by Total Delay & 4 & 3 & 2 & mo \\
\hline
\end{tabular}

\begin{tabular}{|c|c|c|c|c|}
\hline Output Node & 01 & 02 & 03 & 04 \\
\hline Output Values: $i 9=i 14=i 15=1$ & 1.0000 & 1.0000 & 1.0000 & 1.0000 \\
\hline Output Rankings: i $9=i 14=i 15=1$ & 1 & 1 & 1 & $\sqrt{1} . \%$ \\
\hline
\end{tabular}

\begin{tabular}{|l|c|c|c|c|}
\hline Output Node & 01 & 02 & 03 & 04 \\
\hline Output Values: $i 9=i 14=i 15=0.33$ & 0.0604 & 0.0346 & 0.0196 & 0.0178 \\
\hline Output Rankings: $i 9=i 14=i 15=0.33$ & 4 & 3 & 2 & . \\
\hline
\end{tabular}


methods were tested, the neural network correctly selected the first choice route for two of the destination nodes and all three nodes based on total delay. When the input activation was set at 1 , output values were close to saturation; when the input activation was normalized, output values were considerable lower.

Test \#6 is an example of performing a decision from a 4 output originating node (Node 3) to 3 destination nodes (Nodes 9, 14, and 15) with similar output rankings. The results of the test are provided in Table 4.8, with output values of rank 1 shaded for comparison purposes. The results of this test were similar to that of Test \#4; when the input activations were set at 1 , saturation of the outputs occurred, and when the input activations were normalized, very small output activations were produced. In this case, the output rankings were the same as the output rankings based on total delay, and the network selected the correct output.

\subsubsection{Summary of generalization tests}

In reviewing the performance of the neural networks in performing generalization decisions, the results were inconsistent. While some networks correctly determined output rankings, other networks produced incorrect output rankings and selected incorrect routes. In all but one test routing mismatches between the measured rankings and the output rankings based on total delay were off by a value of 1 , the same level of routing mismatches that occurred in the single node routing tests performed in earlier sections. Based on this performance, the neural networks could be used to perform generalized routing decisions for restoration purposes (where rank 1/rank 2 mismatches could be ignored) but the inconsistent results found in the small set of of tests conducted is not a significantly large sample to properly support the above conclusion. More testing of these types of decisions, and investigation into the neural network behaviour found in Tests \#4 and \#6, is required before firm conclusions could be made. 


\section{Chapter 5}

\section{Conclusions and recommendations}

\subsection{Conclusions}

A number of conclusions have been drawn from the work presented in this thesis. They are:

- Several approaches can be considered in mapping telecommunications routing problems into the area of neural networks. By studying the concepts of network routing from the perspective of current routing methods and routing theory, routing patterns or relationships can be extracted for study. In this thesis, a distributed routing routing method based on a time based ranking system was selected for neural network simulation and analysis.

- Experimentation with various neural network variables uncovered differences in the capabilities of neural networks to learn routing information. In this thesis, modifications to the structure of the neural network and to the learning method demonstrated that routing performance could be improved.

- The preparation of the training set information can significantly affect the routing performance of the tested neural networks. The quantization of routing rankings tested in this thesis resulted in poorer learning of the actual rank values but improved learning of the ranking order.

- Trained neural networks, combined with a postprocessing winnertake-all layer, can be used to perform distributed routing. When the set of neural networks was trained with routing information from a sample network, the neural networks performed the specific individual routing tasks with a high degree of efficiency under a number of different network routing conditions. The neural networks were also able to perform a number of generalized routing decisions, but the results of the generalized routing tests were not as 
conclusive as those of the individual routing analysis; a potential explanation for this is that the training sets for the neural networks did not contain any generalizing information.

\subsection{Recommendations}

Based on the work performed, there are a number of recommendations for areas of further investigation. These are:

- Extension of the routing method to more generalized routing problems.

- The performance of the neural networks could be tested with other learning rules such as Hebbian learning or counterpropagation.

- Routing performance can be tested using other neural network topologies. The thesis demonstrated that 8 neurons per hidden layer performed best of the three topologies considered, but other topologies with different number of neurons in each layer are possible. Neural network topologies with 4 and 10 neurons per hidden layer are examples of the different topologies considered for potential study, and the removal of the input neuron representing the originating node can be investigated to evaluate any effects on neural network performance.

- A study of features extracted by the neural network's hidden layers can be performed to determine their relationships to the routing network under study.

- Further testing in the area of generalization can be performed to more fully understand how the networks perform this function, and investigations into how generalized routing information could be incorporated into the training set and neural networks can be performed.

- The neural networks in this thesis were studied using software simulations on a general purpose computer. One possible extension of this work would be to implement the neural networks in silicon to test actual routing performance. 
References 


\section{References}

[1] D.W. Tank and J.J. Hopfield. Collective Computation in Neuronlike Circuits. Scientific American, September 1987, pp. 104-114.

[2] T. Kohonen. An Introduction to Neural Computing. Neural Networks, Volume 1, 1988, pp. 3-16.

[3] G.F. Watson coordinator. $\Delta V=0.7 V=85000$ irate travelers IEEE Spectrum, Volume 29, Number 2, February 1992, p. 54.

[4] G. Zorpette editor.Keeping the phone lines open. IEEE Spectrum, Volume 26, Number 6, June 1989, pp. 32-36.

[5] F. Ellefson. Migration to network restoration. Alberta Telecommunications Research Center conference on Trends in Network Restoration, Edmonton, Alberta. May 24-25, 1990.

[6] F. Corsale Survivable Transport Networks. Alberta Telecommunications Research Center conference on Trends in Network Restoration, Edmonton, Alberta. May 24-25, 1990.

[7] C.H. Yang and S. Hasegawa. FITNESS: Failure Inmmunization Technology for Network Service Survivability. IEEE Global Telecommunications Conference (Globecom) 1988, pp. $1549-1554$.

[8] W.D. Grover, B.D. Venables, M.H. MacGregor, and J.H. Sandham. Development and Performance Assessment of a Distributed Asynchronous Protocol for Real-Time Network Restoration. IEEE Journal on Selected Areas of Communications, Volume 9, Number 1 January 1991, pp.112-125.

[9] N. Deo and C. Pang. Shortest path algorithms: Taxonomy and annotation. Networks, Volume 14, 1984, pp. 275-323.

[10] E.W. Dijkstra. A Note on Two Problems in Connexion with Graphs. Numerishe Mathematik 1, 1959, pp. 269-271.

[11] G. Fahner. An Algorithm-Structured Neural Net for the Shortest-Path Problem, IEEE International Joint Conference on Neural Networks (IJCNN) 1991, pp.I 153-158.

[12] A. Ephremides and S. Verdu. Control and Optimization Methods in Communication Network Problems. IEEE Transactions on Automatic Control, Vol 34, No. 9, September 1989, pp. 930-941.

[13] T.X. Brown. Neural Networks for Switching. IEEE Communications Magazine, November 1989 pp. 72-81.

[14] P.J.W. Melsa, J.B. Kenney, and C.E. Rohrs. A neural network solution for call routing with preferential call placentent. IEEE Global Telecommunications Conference (Globecom) 1990, pp. 1377-1381.

[15] A. Hiramatsu. ATM communications network control by neural network. IEEE 
International Joint Conference on Neural Networks (IJCNN) 1989, pp. 1-259-266.

[16] A. Hiramatsu. Integration of ATM call admission control and link capacity control by distributed netural networks. IEEE Global Telecommunications Conference (Globecom) 1990, pp. 1382-1386.

[17] A. Chugo, W. Sotelo, and I. lida. Holonic Routing Scheme based on Neural Computations. IEEE Global Telecommunications Conference (Globecom) 1990, pp. 13661370 .

[18] G. Frisiani, T. Parisini, L. Siccardi, and R. Zoppoli. Team theory and backpropagation for dynamic routing in communication networks. IEEE International Joint Conference on Neural Networks (IJCNN) 1991, pp. I-325-334.

[19] H.E. Rausch and T. Winarske. Neural Networks for Routing Communications Traffic. IEEE Control Systems Magazine, Volume 8, Issue 2, April 1988, pp. 26-31.

[20] J.E. Jensen, M.A. Eshera, and S.C. Barash. Neural Network Controller for Adaptive $R$ outing in Survivable Communications Networks. IEEE International Joint Conference on Neural Networks (IJCNN) 1990, pp.II 29-36

[21] G.E. Hinton. Connectionist Learning Procedures Artificial Intelligence, Volume 40, Issues 1-3, September 1989, pp. 285-235.

[22] R.P. Lippman. An introduction to Computing with Neural Nets IEEE ASSP Magazine, April 1987, pp. 4-22.

[23] M. Jensen, S. Kennedy, and G. Blanston. MacBrain 3.0 User Manual.

[24] H.C. Card. Lecture notes for University of Manitoba course 24.723 "Neural Networks", Spring 1990. Portions of course notes (C) G.E. Hinton 1989. 
Appendix A:

Training macros and sets 


\section{Icon based representation of the $V 1$ Macro}

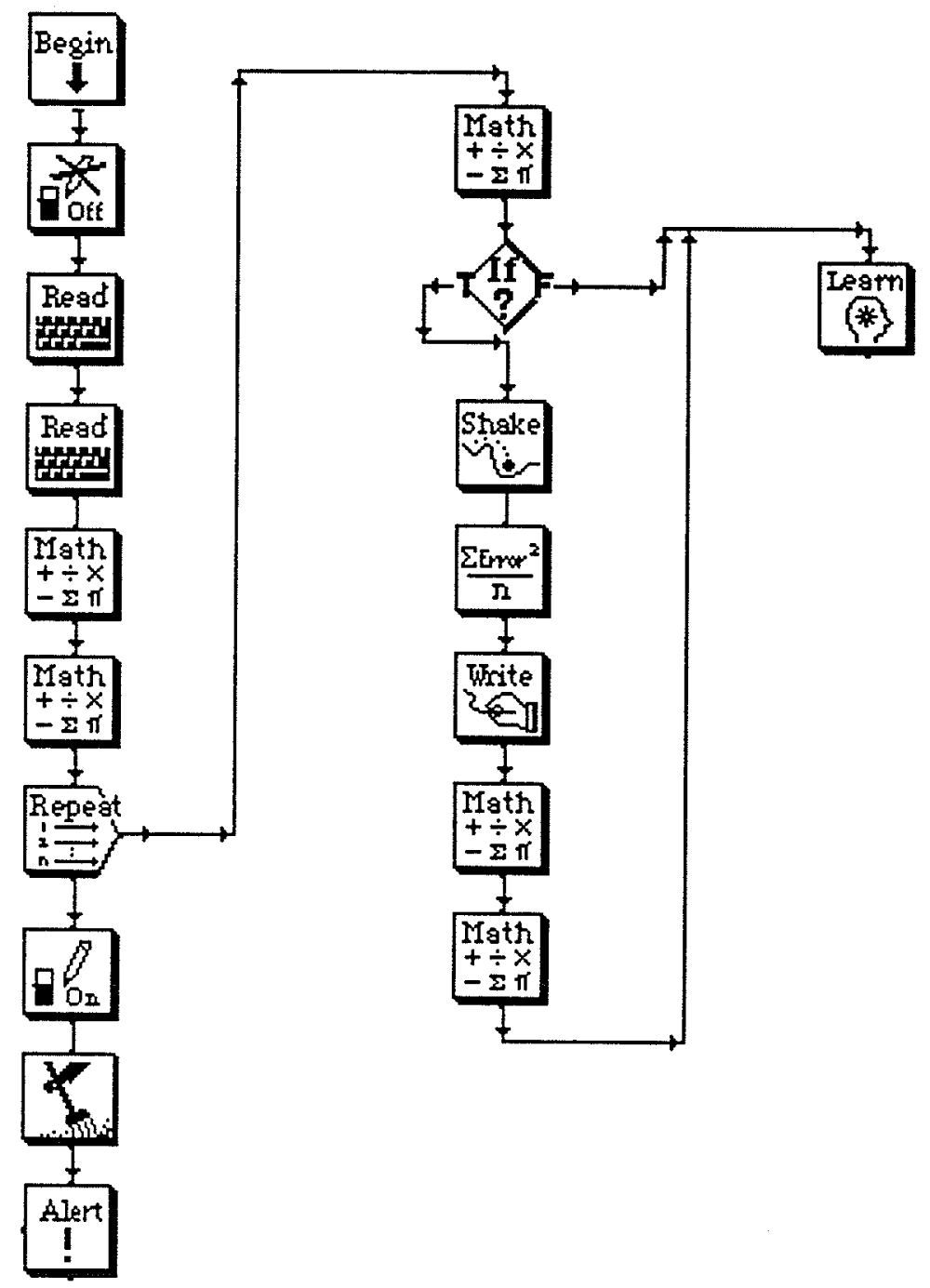

Page A.1 


$$
\begin{gathered}
\text { Icon based representation } \\
\text { of the V2 Macro }
\end{gathered}
$$

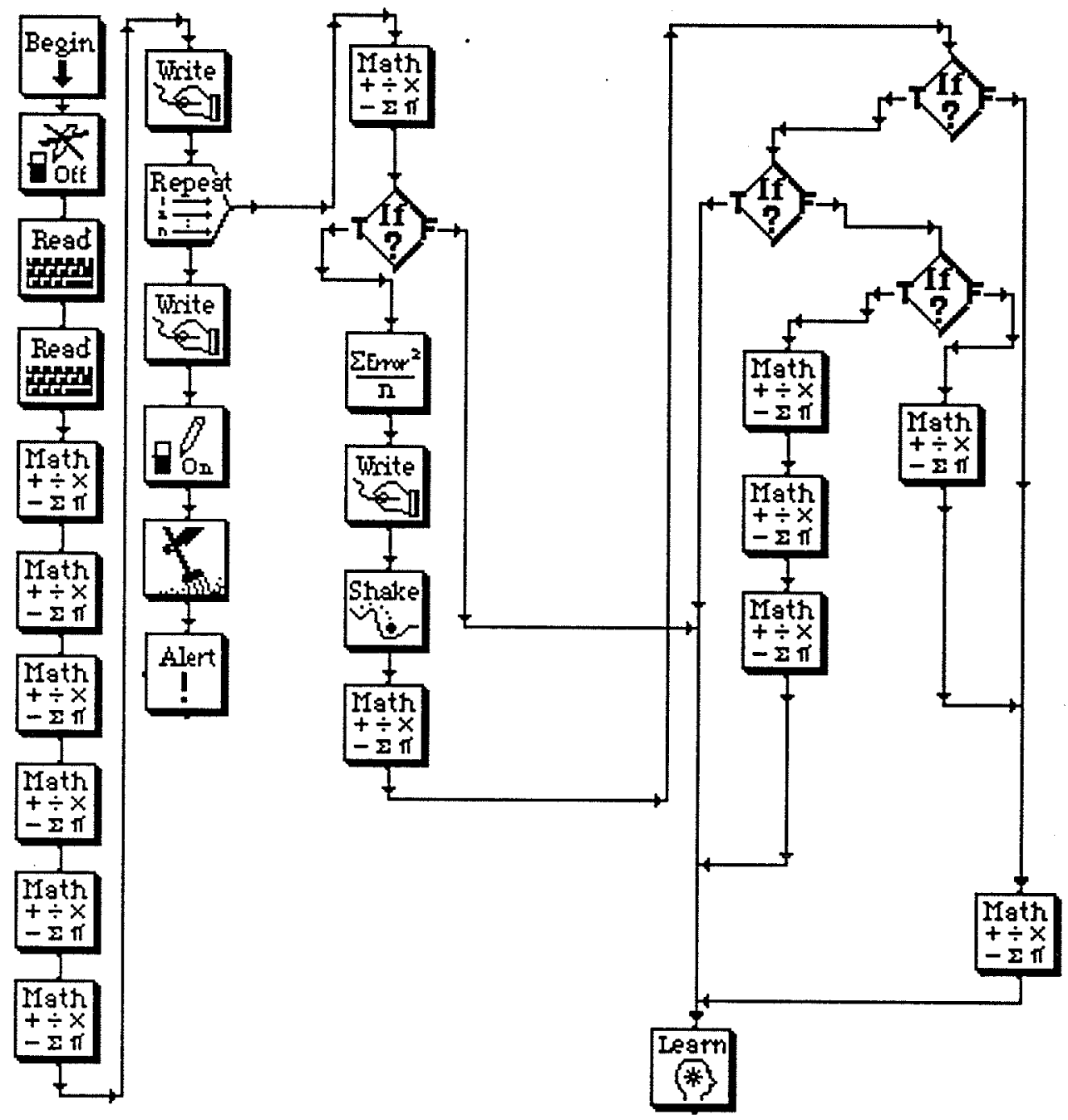

Page A.2 
Pattern File for Node 2 of Sample Network

\begin{tabular}{|c|c|c|c|c|c|c|c|c|c|c|c|c|c|c|c|c|c|c|}
\hline \multirow{2}{*}{$\begin{array}{l}\text { Destination } \\
\text { Node }\end{array}$} & \multicolumn{16}{|c|}{ Input Vector } & \multicolumn{2}{|c|}{ Output Vector } \\
\hline & i1 & $i 2$ & i3 & $i 4$ & i5 & $i 6$ & $i 7$ & $i 8$ & i9 & $i 10$ & $i 11$ & $i 12$ & $i 13$ & $i 14$ & $i 15$ & $i 16$ & 01 & 02 \\
\hline 1 & 1 & 0 & 0 & 0 & 0 & 0 & 0 & 0 & 0 & 0 & 0 & 0 & 0 & 0 & 0 & 0 & 0.2569 & 0.3986 \\
\hline 2 & 0 & 1 & 0 & 0 & 0 & 0 & 0 & 0 & 0 & 0 & 0 & 0 & 0 & 0 & 0 & 0 & 0.0000 & 0.0000 \\
\hline 3 & 0 & 0 & 1 & 0 & 0 & 0 & 0 & 0 & 0 & 0 & 0 & 0 & 0 & 0 & 0 & 0 & 0.1245 & 0.2663 \\
\hline 4 & 0 & 0 & 0 & 1 & 0 & 0 & 0 & 0 & 0 & 0 & 0 & 0 & 0 & 0 & 0 & 0 & 0.3486 & 0.3966 \\
\hline 5 & 0 & 0 & 0 & 0 & 1 & 0 & 0 & 0 & 0 & 0 & 0 & 0 & 0 & 0 & 0 & 0 & 0.4146 & 0.4924 \\
\hline 6 & 0 & 0 & 0 & 0 & 0 & 1 & 0 & 0 & 0 & 0 & 0 & 0 & 0 & 0 & 0 & 0 & 0.2335 & 0.1573 \\
\hline 7 & 0 & 0 & 0 & 0 & 0 & 0 & 1 & 0 & 0 & 0 & 0 & 0 & 0 & 0 & 0 & 0 & 0.249 & 0.297 \\
\hline 8 & 0 & 0 & 0 & 0 & 0 & 0 & 0 & 1 & 0 & 0 & 0 & 0 & 0 & 0 & 0 & 0 & 0.3588 & 0.4068 \\
\hline 9 & 0 & 0 & 0 & 0 & 0 & 0 & 0 & 0 & 1 & 0 & 0 & 0 & 0 & 0 & 0 & 0 & 0.4618 & 0.5097 \\
\hline 10 & 0 & 0 & 0 & 0 & 0 & 0 & 0 & 0 & 0 & 1 & 0 & 0 & 0 & 0 & 0 & 0 & 0.3524 & 0.2762 \\
\hline 11 & 0 & 0 & 0 & 0 & 0 & 0 & 0 & 0 & 0 & 0 & 1 & 0 & 0 & 0 & 0 & 0 & 0.3588 & 0.3793 \\
\hline 12 & 0 & 0 & 0 & 0 & 0 & 0 & 0 & 0 & 0 & 0 & 0 & 1 & 0 & 0 & 0 & 0 & 0.4776 & 0.4981 \\
\hline 13 & 0 & 0 & 0 & 0 & 0 & 0 & 0 & 0 & 0 & 0 & 0 & 0 & 1 & 0 & 0 & 0 & 0.6385 & 0.659 \\
\hline 14 & 0 & 0 & 0 & 0 & 0 & 0 & 0 & 0 & 0 & 0 & 0 & 0 & 0 & 1 & 0 & 0 & 0.4405 & 0.3643 \\
\hline 15 & 0 & 0 & 0 & 0 & 0 & 0 & 0 & 0 & 0 & 0 & 0 & 0 & 0 & 0 & 1 & 0 & 0.4942 & 0.4733 \\
\hline 16 & 0 & 0 & 0 & 01 & 0 & 0 & 0 & 0 & 0 & 0 & 0 & 0 & 0 & 0 & 0 & 1 & 0.6745 & 0.6536 \\
\hline
\end{tabular}


Pattern File for Node 9 of the Sample Network

\begin{tabular}{|c|c|c|c|c|c|c|c|c|c|c|c|c|c|c|c|c|c|c|c|c|}
\hline \multirow{2}{*}{$\begin{array}{c}\text { Destination } \\
\text { Node }\end{array}$} & \multicolumn{16}{|c|}{ Input Vector } & \multicolumn{4}{|c|}{ Output Vector } \\
\hline & $i 1$ & $i 2$ & i3 & $\mathrm{i4}$ & $i 5$ & i6 & $i 7$ & $i 8$ & i9 & $i 10$ & $i 11$ & $i 12$ & $i 13$ & $i 14$ & $i 15$ & $i 16$ & 01 & 02 & 03 & 04 \\
\hline i1 & 1 & 0 & 0 & 0 & 0 & 0 & 0 & 0 & 0 & 0 & 0 & 0 & 0 & 0 & 0 & 0 & 0.3708 & 0.3838 & 0.5985 & 0.8306 \\
\hline 12 & 0 & 1 & 0 & 0 & 0 & 0 & 0 & 0 & 0 & 0 & 0 & 0 & 0 & 0 & 0 & 0 & 0.6027 & 0.4619 & 0.6191 & 0.8512 \\
\hline i3 & 0 & 0 & 1 & 0 & 0 & 0 & 0 & 0 & 0 & 0 & 0 & 0 & 0 & 0 & 0 & 0 & 0.5031 & 0.3623 & 0.5195 & 0.7516 \\
\hline$i 4$ & 0 & 0 & 0 & 1 & 0 & 0 & 0 & 0 & 0 & 0 & 0 & 0 & 0 & 0 & 0 & 0 & 0.3088 & 0.2418 & 0.4565 & 0.6886 \\
\hline 15 & 0 & 0 & 0 & 0 & 1 & 0 & 0 & 0 & 0 & 0 & 0 & 0 & 0 & 0 & 0 & 0 & 0.213 & 0.3376 & 0.5523 & 0.7844 \\
\hline$i 6$ & 0 & 0 & 0 & 0 & 0 & 1 & 0 & 0 & 0 & 0 & 0 & 0 & 0 & 0 & 0 & 0 & 0.5481 & 0.3776 & 0.5072 & 0.7393 \\
\hline$i 7$ & 0 & 0 & 0 & 0 & 0 & 0 & 1 & 0 & 0 & 0 & 0 & 0 & 0 & 0 & 0 & 0 & 0.4084 & 0.2379 & 0.395 & 0.6271 \\
\hline$i 8$ & 0 & 0 & 0 & 0 & 0 & 0 & 0 & 1 & 0 & 0 & 0 & 0 & 0 & 0 & 0 & 0 & 0.4225 & 0.1281 & 0.3428 & 0.5749 \\
\hline i9 & 0 & 0 & 0 & 0 & 0 & 0 & 0 & 0 & 1 & 0 & 0 & 0 & 0 & 0 & 0 & 0 & 0.0000 & 0.0000 & 0.0000 & 0.0000 \\
\hline$i 10$ & 0 & 0 & 0 & 0 & 0 & 0 & 0 & 0 & 0 & 1 & 0 & 0 & 0 & 0 & 0 & 0 & 0.6212 & 0.3761 & 0.3884 & 0.6205 \\
\hline$i 11$ & 0 & 0 & 0 & 0 & 0 & 0 & 0 & 0 & 0 & 0 & 1 & 0 & 0 & 0 & 0 & 0 & 0.5181 & 0.273 & 0.2853 & 0.5174 \\
\hline$i 12$ & 0 & 0 & 0 & 0 & 0 & 0 & 0 & 0 & 0 & 0 & 0 & 1 & 0 & 0 & 0 & 0 & 0.5989 & 0.3045 & 0.1664 & 0.3985 \\
\hline$i 13$ & 0 & 0 & 0 & 0 & 0 & 0 & 0 & 0 & 0 & 0 & 0 & 0 & 1 & 0 & 0 & 0 & 0.7597 & 0.4653 & 0.3272 & 0.2377 \\
\hline$i 14$ & 0 & 0 & 0 & 0 & 0 & 0 & 0 & 0 & 0 & 0 & 0 & 0 & 0 & 1 & 0 & 0 & 0.663 & 0.4179 & 0.3851 & 0.6172 \\
\hline$i 15$ & 0 & 0 & 0 & 0 & 0 & 0 & 0 & 0 & 0 & 0 & 0 & 0 & 0 & 0 & 1 & 0 & 0.6536 & 0.4085 & 0.2762 & 0.5083 \\
\hline$i 16$ & 0 & 01 & 0 & 0 & 0 & 0 & 0 & 0 & 0 & 0 & 0 & 0 & 0 & 0 & 0 & 1 & 0.8046 & 0.5102 & 0.3712 & 0.3929 \\
\hline
\end{tabular}


Pattern File for Node 11 of the Sample Network

\begin{tabular}{|c|c|c|c|c|c|c|c|c|c|c|c|c|c|c|c|c|c|c|c|c|c|c|}
\hline \multirow{2}{*}{$\begin{array}{l}\text { Destination } \\
\text { Node }\end{array}$} & \multicolumn{16}{|c|}{ Input Vector } & \multicolumn{6}{|c|}{ Output Vector } \\
\hline & $i 1$ & 12 & i3 & $i 4$ & $i 5$ & 16 & 17 & $i 8$ & i9 & $i 10$ & $i 11$ & $i 12$ & $i 13$ & $i 14$ & i15 & $\lcm{i 16}$ & 01 & 02 & 03 & 04 & 05 & 06 \\
\hline 1 & 1 & 0 & 0 & 0 & 0 & 0 & 0 & 0 & 0 & 0 & 0 & 0 & 0 & 0 & 0 & 0 & 0.3763 & 0.4256 & 0.4882 & 0.5759 & 0.6182 & 0.7023 \\
\hline 2 & 0 & 1 & 0 & 0 & 0 & 0 & 0 & 0 & 0 & 0 & 0 & 0 & 0 & 0 & 0 & 0 & 0.3588 & 0.5037 & 0.3793 & 0.6541 & 0.5092 & 0.6088 \\
\hline 3 & 0 & 0 & 1 & 0 & 0 & 0 & 0 & 0 & 0 & 0 & 0 & 0 & 0 & 0 & 0 & 0 & 0.2592 & 0.4041 & 0.3559 & 0.5545 & 0.4859 & 0.5854 \\
\hline 4 & 0 & 0 & 0 & 1 & 0 & 0 & 0 & 0 & 0 & 0 & 0 & 0 & 0 & 0 & 0 & 0 & 0.2343 & 0.2836 & 0.4862 & 0.4339 & 0.6162 & 0.5603 \\
\hline 5 & 0 & 0 & 0 & 0 & 1 & 0 & 0 & 0 & 0 & 0 & 0 & 0 & 0 & 0 & 0 & 0 & 0.3301 & 0.3794 & 0.582 & 0.4732 & 0.712 & 0.5997 \\
\hline 6 & 0 & 0 & 0 & 0 & 0 & 1 & 0 & 0 & 0 & 0 & 0 & 0 & 0 & 0 & 0 & 0 & 0.2745 & 0.4194 & 0.247 & 0.5696 & 0.3769 & 0.4765 \\
\hline 7 & 0 & 0 & 0 & 0 & 0 & 0 & 1 & 0 & 0 & 0 & 0 & 0 & 0 & 0 & 0 & 0 & 0.1348 & 0.2797 & 0.3867 & 0.43 & 0.5166 & 0.5564 \\
\hline 8 & 0 & 0 & 0 & 0 & 0 & 0 & 0 & 1 & 0 & 0 & 0 & 0 & 0 & 0 & 0 & 0 & 0.2445 & 0.1699 & 0.4964 & 0.3203 & 0.565 & 0.4467 \\
\hline 9 & 0 & 0 & 0 & 0 & 0 & 0 & 0 & 0 & 1 & 0 & 0 & 0 & 0 & 0 & 0 & 0 & 0.3476 & 0.273 & 0.5995 & 0.2853 & 0.53 & 0.4117 \\
\hline 10 & 0 & 0 & 0 & 0 & 0 & 0 & 0 & 0 & 0 & 1 & 0 & 0 & 0 & 0 & 0 & 0 & 0.3933 & 0.5382 & 0.1281 & 0.4871 & 0.2944 & 0.3939 \\
\hline 11 & 0 & 0 & 0 & 0 & 0 & 0 & 0 & 0 & 0 & 0 & 1 & 0 & 0 & 0 & 0 & 0 & 0.0000 & 0.0000 & 0.0000 & 0.0000 & 0.0000 & 0.0000 \\
\hline 12 & 0 & 0 & 0 & of & 0 & 0 & 0 & 0 & 0 & 0 & 0 & 1 & 0 & 0 & 0 & 0 & 0.4209 & 0.3463 & 0.4713 & 0.1439 & 0.3886 & 0.2703 \\
\hline 13 & 0 & 0 & 0 & 요 & 0 & 0 & 0 & 0 & 0 & 0 & 0 & 0 & 1 & 0 & 0 & 0 & 0.5603 & 0.4857 & 0.6321 & 0.3047 & 0.5494 & 0.4311 \\
\hline 14 & 0 & 0 & 0 & 0 & 0 & 0 & 0 & 0 & 0 & 0 & 0 & 0 & 0 & 1 & 0 & 0 & 0.4815 & 0.6331 & 0.2526 & 0.3626 & 0.1699 & 0.2695 \\
\hline 15 & 0 & 0 & 0 & 0 & 0 & 0 & 0 & 0 & 0 & 0 & 0 & 0 & 0 & 0 & 1 & 0 & 0.5307 & 0.456 & 0.3615 & 0.2536 & 0.2789 & 0.1605 \\
\hline 16 & 0 & 01 & 0 & 0 & 0 & 0 & 0 & 0 & 0 & 0 & 0 & 0 & 0 & 0 & 0 & 1 & 0.6266 & 0.552 & 0.5418 & 0.3496 & 0.4591 & 0.3408 \\
\hline
\end{tabular}


Appendix B:

Single node modelling results 
Training Results for V1.2.06.2

(Network 2.06.2, V1 Macro)

\begin{tabular}{|c|c|c|c|c|c|c|}
\hline \multirow[t]{2}{*}{ Destination } & \multicolumn{3}{|c|}{ Neuron o1 (Route 3) } & \multicolumn{3}{|c|}{ Neuron o2 (Route 6) } \\
\hline & Target & Meas & Diff & Target & Meas & Diff \\
\hline i 1 & \multirow[t]{2}{*}{0.2569} & 0.30273 & -0.0458 & \multirow[t]{2}{*}{0.3986} & 0.31941 & 0.07919 \\
\hline i 2 & & 0.04306 & -0.0431 & & 0.04654 & -0.0465 \\
\hline i3 & 0.1245 & 0.04622 & 0.07828 & 0.2663 & 0.05003 & 0.21627 \\
\hline ¡ 4 & 0.3486 & 0.34681 & 0.00179 & 0.3966 & 0.36605 & 0.03055 \\
\hline i5 & 0.4146 & 0.43077 & -0.0162 & 0.4924 & 0.45044 & 0.04196 \\
\hline$i 6$ & 0.2335 & 0.041 & 0.1925 & 0.1573 & 0.04433 & 0.11297 \\
\hline i 7 & 0.249 & 0.06095 & 0.18805 & 0.297 & 0.06602 & 0.23098 \\
\hline i 8 & 0.3588 & 0.36095 & -0.0021 & 0.4068 & 0.38049 & 0.02631 \\
\hline i9 & 0.4618 & 0.45955 & 0.00225 & 0.5097 & 0.4796 & 0.0301 \\
\hline$i 10$ & 0.3524 & 0.29049 & 0.06191 & 0.2762 & 0.30855 & -0.0324 \\
\hline$i 11$ & 0.3588 & 0.3472 & 0.0 & 0.3793 & 0.3667 & 0.0126 \\
\hline $\mathrm{i} 12$ & 0.4776 & 0.46848 & 0.00912 & 0.4981 & 0.48932 & 0.00878 \\
\hline 113 & 0.6385 & 0.63023 & 0.00827 & 0.659 & 0.64821 & 0.01079 \\
\hline i14 & 0.4405 & 0.38725 & 0.05325 & 0.3643 & 0.3994 & -0.0351 \\
\hline$i 15$ & 0.4942 & 0.46577 & 0.02843 & 0.4733 & 0.47587 & -0.0026 \\
\hline$i 16$ & 0.6745 & 0.64238 & 0.03212 & 0.6536 & 0.65873 & -0.0051 \\
\hline \multirow{4}{*}{$\begin{array}{l}\text { Neuron } \\
\text { Results }\end{array}$} & \multicolumn{2}{|c|}{ Average |Diff (Full) } & 0.04842 & \multicolumn{2}{|c|}{ Average |Diff| (Full) } & 0.05764 \\
\hline & \multicolumn{2}{|c|}{ Average |Diff (Mod) } & 0.04878 & \multicolumn{2}{|c|}{ Average |Diff| (Mod) } & 0.05838 \\
\hline & \multicolumn{2}{|c|}{ Std Dev |Diff| (Full) } & 0.06003 & \multicolumn{2}{|c|}{ Std Dev |Diff| (Full) } & 0.07077 \\
\hline & \multicolumn{2}{|c|}{ Std Dev |Diff| (Mod) } & 0.06212 & \multicolumn{2}{|c|}{ Std Dev |Diff| (Mod) } & 0.07319 \\
\hline
\end{tabular}

\begin{tabular}{|c|c|c|c|c|}
\hline \multirow[t]{2}{*}{$\begin{array}{l}\text { Network } \\
\text { Results }\end{array}$} & \begin{tabular}{|l|l|} 
Average $\mid$ Diff $\mid$ & (Full) \\
Average $\mid$ Diff $\mid$ & (Mod)
\end{tabular} & $\frac{0.05303}{0.05358}$ & $\begin{array}{l}\text { Std Dev |Diff| } \\
\text { Std Full) }\end{array}$ & 0.06472 \\
\hline & [Average [Ditt (Mod) & 0.05358 & Std Dev |Diff| (Mod) & 0.06688 \\
\hline
\end{tabular}


Training Results for V1.2.08.2

(Network 2.08.2, V1 Macro)

\begin{tabular}{|c|c|c|c|c|c|c|}
\hline \multirow[t]{2}{*}{ Destination } & \multicolumn{3}{|c|}{ Neuron o1 (Route 3) } & \multicolumn{3}{|c|}{ Neuron 02 (Route 6) } \\
\hline & Target & Meas & Diff & Target & Meas & Diff \\
\hline$i 1$ & 0.2569 & 0.28168 & -0.0248 & 0.3986 & 0.30242 & 0.09618 \\
\hline$i 2$ & & 0.0715 & -0.0715 & & 0.07721 & -0.0772 \\
\hline i3 & 0.1245 & 0.07042 & 0.05408 & 0.2663 & 0.07659 & 0.18971 \\
\hline ¡ 4 & 0.3486 & 0.33394 & 0.01466 & 0.3966 & 0.35496 & 0.04164 \\
\hline i 5 & 0.4146 & 0.42424 & -0.0096 & 0.4924 & 0.44666 & 0.04574 \\
\hline i 6 & 0.2335 & 0.06315 & 0.17035 & 0.1573 & 0.06845 & 0.08885 \\
\hline i7 & 0.249 & 0.07516 & 0.17384 & 0.297 & 0.08133 & 0.21567 \\
\hline i 8 & 0.3588 & 0.35021 & 0.00859 & 0.4068 & 0.37191 & 0.03489 \\
\hline i9 & 0.4618 & 0.45184 & 0.00996 & 0.5097 & 0.47508 & 0.03462 \\
\hline$\$ 10$ & 0.3524 & 0.22612 & 0.12628 & 0.2762 & 0.24254 & 0.03366 \\
\hline i11 & 0.3588 & 0.32692 & 0.03188 & 0.3793 & 0.34806 & 0.03124 \\
\hline$i 12$ & 0.4776 & 0.46238 & 0.01522 & 0.4981 & 0.48504 & 0.01306 \\
\hline$i 13$ & 0.6385 & 0.62634 & 0.01216 & 0.659 & 0.64764 & 0.01136 \\
\hline$i 14$ & 0.4405 & 0.37257 & 0.06793 & 0.3643 & 0.39235 & -0.0281 \\
\hline$i 15$ & 0.4942 & 0.43674 & 0.05746 & 0.4733 & 0.45938 & 0.01392 \\
\hline$\$ 16$ & 0.6745 & 0.64214 & 0.03236 & 0.6536 & 0.66176 & -0.0082 \\
\hline \multirow{4}{*}{$\begin{array}{l}\text { Neuron } \\
\text { Results }\end{array}$} & \multicolumn{2}{|c|}{ Average |Diff (Full) } & 0.05504 & \multicolumn{2}{|c|}{ Average |Diff | (Full) } & 0.06025 \\
\hline & \multicolumn{2}{|c|}{ Average |Diff $\mid$ (Mod) } & 0.05395 & \multicolumn{2}{|c|}{ Average [Diff] (Mod) } & 0.05912 \\
\hline & \multicolumn{2}{|c|}{ Std Dev |Diff| (Full) } & 0.0555 & \multicolumn{2}{|c|}{\begin{tabular}{|l|l|l|} 
Std Dev & Diff & (Full) \\
\end{tabular}} & 0.06177 \\
\hline & \multicolumn{2}{|c|}{ Std Dev |Diff| (Mod) } & 0.05727 & \multicolumn{2}{|c|}{ Std Dev |Diff| (Mod) } & 0.06377 \\
\hline
\end{tabular}

\begin{tabular}{|c|c|c|c|}
\hline \multirow[t]{2}{*}{$\begin{array}{l}\text { Network } \\
\text { Results }\end{array}$} & \begin{tabular}{|l|l|l|} 
Average & Diff & (Full) \\
Average & IDiff & (Mnd)
\end{tabular} & \begin{tabular}{l|l|l|}
0.05765 & Std Dev |Diff| (Full) \\
0.05653 & Std
\end{tabular} & 0.0 \\
\hline & [Average [Ditt (Mod) & $0.05653 \mid$ Std Dev |Diff $\mid$ (Mod) & 0.05961 \\
\hline
\end{tabular}


Training Results for V1.2.10.2

(Network 2.10.2, V1 Macro)

\begin{tabular}{|c|c|c|c|c|c|c|}
\hline \multirow[t]{2}{*}{ Destination } & \multicolumn{3}{|c|}{ Neuron 01 (Route 3) } & \multicolumn{3}{|c|}{ Nueron o2 (Route 6) } \\
\hline & Target & Meas & Diff & Target & Meas & Diff \\
\hline i1 & 0.2569 & 0.14932 & 0.10758 & 0.3986 & 0.16186 & 0.23674 \\
\hline i2 & & 0.10219 & -0.1022 & & 0.1108 & -0.1108 \\
\hline i3 & 0.1245 & 0.09382 & 0.03068 & 0.2663 & 0.10212 & 0.16418 \\
\hline i 4 & 0.3486 & 0.28185 & 0.06675 & 0.3966 & 0.30104 & 0.09556 \\
\hline$i 5$ & 0.4146 & 0.41347 & 0.00113 & 0.4924 & 0.43604 & 0.05636 \\
\hline i 6 & 0.2335 & 0.08242 & 0.15108 & 0.1573 & 0.08961 & 0.06769 \\
\hline i7 & 0.249 & 0.104 & 0.145 & 0.297 & 0.11283 & 0.18417 \\
\hline i8 & 0.3588 & 0.34716 & 0.01164 & 0.4068 & 0.36798 & 0.03882 \\
\hline i 9 & 0.4618 & 0.43911 & 0.02269 & 0.5097 & 0.46259 & 0.04711 \\
\hline i10 & 0.3524 & 0.10614 & 0.24626 & 0.2762 & 0.11508 & 0.16112 \\
\hline$i 11$ & 0.3588 & 0.32868 & 0.03012 & 0.3793 & 0.35833 & 0.02097 \\
\hline$i 12$ & 0.4776 & 0.45562 & 0.02198 & 0.4981 & 0.47872 & 0.01938 \\
\hline$i 13$ & 0.6385 & 0.624 & 0.0145 & 0.659 & 0.64538 & 0.01362 \\
\hline$i 14$ & 0.4405 & 0.3625 & 0.078 & 0.3643 & 0.3826 & -0.0183 \\
\hline$i 15$ & 0.4942 & 0.43063 & 0.06357 & 0.4733 & 0.45226 & 0.02104 \\
\hline$i 16$ & 0.6745 & 0.63911 & 0.03539 & 0.6536 & 0.65956 & -0.006 \\
\hline \multirow{4}{*}{$\begin{array}{l}\text { Neuron } \\
\text { Results }\end{array}$} & \multicolumn{2}{|c|}{ Average |Diff $\mid$ (Full) } & 0.07054 & \multicolumn{2}{|c|}{\begin{tabular}{|l|l|l|} 
Average & Diff & (Full) \\
\end{tabular}} & 0.07886 \\
\hline & \multicolumn{2}{|c|}{ Average |Diff| (Mod) } & 0.06842 & \multicolumn{2}{|c|}{ Average |Diff| (Mod) } & 0.07673 \\
\hline & \multicolumn{2}{|c|}{ Std Dev |Diff | (Full) } & 0.0663 & \multicolumn{2}{|c|}{ Std Dev |Diff $\mid$ (Full) } & 0.07215 \\
\hline & \multicolumn{2}{|c|}{ Std Dev $\mid$ Diff $\mid$ (Mod) } & 0.06807 & \multicolumn{2}{|c|}{ Std Dev |Diff| (Mod) } & 0.07416 \\
\hline \multirow{2}{*}{$\begin{array}{l}\text { Network } \\
\text { Results }\end{array}$} & \multirow{2}{*}{\multicolumn{2}{|c|}{\begin{tabular}{|l|l|} 
Average & Diff $\mid$ (Full) \\
Average & Diff $\mid$ (Mod) \\
\end{tabular}}} & 0.0747 & \multirow{2}{*}{\multicolumn{2}{|c|}{\begin{tabular}{|l|l|} 
Std Dev |Diff $\mid$ (Full) \\
Std Dev |Diff | (Mod) \\
\end{tabular}}} & 0.06829 \\
\hline & & & 0.07258 & & & 0.07007 \\
\hline
\end{tabular}


Training Results for V1.2.06.4

(Network 2.06.4, V1 Macro)

\begin{tabular}{|c|c|c|c|c|c|c|}
\hline \multirow[t]{2}{*}{ Destination } & \multicolumn{3}{|c|}{ Neuron o1 (Route 5) } & \multicolumn{3}{|c|}{ Neuron o2 (Route 8) } \\
\hline & Target & Meas & Diff & Target & Meas & Diff \\
\hline$i 1$ & 0.3708 & 0.38696 & -0.0162 & 0.3838 & 0.34123 & 0.04257 \\
\hline$i 2$ & 0.6027 & 0.59431 & 0.00839 & 0.4619 & 0.45203 & 0.00987 \\
\hline i 3 & 0.5031 & 0.49084 & 0.01226 & 0.3623 & 0.34667 & 0.01563 \\
\hline 14 & 0.3088 & 0.2663 & 0.0425 & 0.2418 & 0.23695 & 0.00485 \\
\hline i 5 & 0.213 & 0.28783 & -0.0748 & 0.3376 & 0.27743 & 0.06017 \\
\hline 16 & 0.5481 & 0.53388 & 0.01422 & 0.3776 & 0.36776 & 0.00984 \\
\hline$i 7$ & 0.4084 & 0.33077 & 0.07763 & 0.2379 & 0.24452 & -0.0066 \\
\hline i 8 & 0.4225 & 0.26476 & 0.15774 & 0.1281 & 0.2078 & -0.0797 \\
\hline i 9 & & 0.03653 & -0.0365 & & 0.02367 & -0.0237 \\
\hline$i 10$ & 0.6212 & 0.62441 & -0.0032 & 0.3761 & 0.37954 & -0.0034 \\
\hline$i 11$ & 0.5181 & 0.5127 & 0.0054 & 0.273 & 0.28933 & -0.0163 \\
\hline$i 12$ & 0.5989 & 0.58511 & 0.01379 & 0.3045 & 0.31116 & -0.0067 \\
\hline$i 13$ & 0.7597 & 0.78442 & -0.0247 & 0.4653 & 0.46794 & -0.0026 \\
\hline$i 14$ & 0.663 & 0.67004 & -0.007 & 0.4179 & 0.41063 & 0.00727 \\
\hline i15 & 0.6536 & 0.67837 & -0.0248 & 0.4085 & 0.38525 & 0.02325 \\
\hline$\$ 16$ & 0.8046 & 0.79761 & 0.00699 & 0.5102 & 0.4862 & 0.024 \\
\hline \multirow{4}{*}{$\begin{array}{l}\text { Neuron } \\
\text { Results }\end{array}$} & \multicolumn{2}{|c|}{ Average |Diff| (Full) } & 0.03289 & \multicolumn{2}{|c|}{ Average |Diff| (Full) } & 0.02103 \\
\hline & \multicolumn{2}{|c|}{ Average |Diff| (Mod) } & 0.03264 & \multicolumn{2}{|c|}{ Average |Diff| (Mod) } & 0.02086 \\
\hline & \multirow{2}{*}{\multicolumn{2}{|c|}{$\begin{array}{l}\text { Std Dev } \mid \text { Diff } \mid \text { (Full) } \\
\text { Std Dev Diff } \mid \text { (Mnd }\end{array}$}} & 0.04048 & \multirow{2}{*}{\multicolumn{2}{|c|}{\begin{tabular}{|l|} 
Std Dev $\mid$ Diff $\mid$ (Full) \\
Std Dev $\mid$ Diff $\mid$ (Mod)
\end{tabular}}} & 0.02202 \\
\hline & & iff] (Mod) & 0.04189 & & & 0.02278 \\
\hline
\end{tabular}

\begin{tabular}{|c|c|c|c|c|c|c|}
\hline \multirow[t]{2}{*}{ Destination } & \multicolumn{3}{|c|}{ Neuron o3 (Route 12) } & \multicolumn{3}{|c|}{ Neuron o4 (Route 13) } \\
\hline & Target & Meas & Diff & Target & Meas & Diff \\
\hline i1 & 0.5985 & 0.59328 & 0.00522 & 0.8306 & 0.83464 & -0.004 \\
\hline i2 & 0.6191 & 0.61651 & 0.00259 & 0.8512 & 0.83803 & 0.01317 \\
\hline i3 & 0.5195 & 0.5021 & 0.0174 & 0.7516 & 0.76498 & -0.0134 \\
\hline ¡ 4 & 0.4565 & 0.44198 & 0.01452 & 0.6886 & 0.70348 & -0.0149 \\
\hline i5 & 0.5523 & 0.53303 & 0.01927 & 0.7844 & 0.78615 & -0.0018 \\
\hline i 6 & 0.5072 & 0.49192 & 0.01528 & 0.7393 & 0.74378 & -0.0045 \\
\hline i 7 & 0.395 & 0.38908 & 0.00592 & 0.6271 & 0.64714 & -0.02 \\
\hline i8 & 0.3428 & 0.34296 & -0.0002 & 0.5749 & 0.58669 & -0.0118 \\
\hline i 9 & & 0.02331 & -0.0233 & & 0.03318 & -0.0332 \\
\hline 110 & 0.3884 & 0.38312 & 0.00528 & 0.6205 & 0.58901 & 0.03149 \\
\hline$i 11$ & 0.2853 & 0.2917 & -0.0064 & 0.5174 & 0.47513 & 0.04227 \\
\hline$i 12$ & 0.1664 & 0.2295 & -0.0631 & 0.3985 & 0.31962 & 0.07888 \\
\hline$\lceil 13$ & 0.3272 & 0.24207 & 0.08513 & 0.2377 & 0.24794 & -0.0102 \\
\hline$i 14$ & 0.3851 & 0.39052 & -0.0054 & 0.6172 & 0.58445 & 0.03275 \\
\hline$i 15$ & 0.2762 & 0.30626 & -0.0301 & 0.5083 & 0.44889 & 0.05941 \\
\hline$i 16$ & 0.3712 & 0.33132 & 0.03988 & 0.3929 & 0.43999 & -0.0471 \\
\hline \multirow{4}{*}{$\begin{array}{l}\text { Neuron } \\
\text { Results }\end{array}$} & \multicolumn{2}{|c|}{ Average [Diff (Full) } & 0.02118 & \multicolumn{2}{|c|}{\begin{tabular}{|l|l|} 
Average & Diff \\
(Full)
\end{tabular}} & 0.02618 \\
\hline & \multicolumn{2}{|c|}{ Average $\mid$ Diff $\mid$ (Mod) } & 0.02104 & \multicolumn{2}{|c|}{ Average |Diff $\mid$ (Mod) } & 0.02571 \\
\hline & \multicolumn{2}{|c|}{ Std Dev |Diff| (Full) } & 0.02362 & \multicolumn{2}{|c|}{ Std Dev (Diff $\mid$ (Full) } & 0.02193 \\
\hline & \multicolumn{2}{|c|}{ Std Dev |Diff $\mid$ (Mod) } & 0.02445 & \multicolumn{2}{|c|}{ Std Dev |Diff| (Mod) } & 0.02262 \\
\hline
\end{tabular}

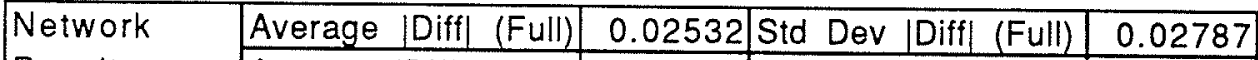

\begin{tabular}{|l|l|l|l|l|l|}
\hline Results & Average & Diffl (Mod) & 0.02506 & Std Dev & Diff \\
(Mod) & 0.02874 \\
\hline
\end{tabular} 
Training Results for V1.2.08.4

(Network 2.08.4, V1 Macro)

\begin{tabular}{|c|c|c|c|c|c|c|}
\hline \multirow[t]{2}{*}{ Destination } & \multicolumn{3}{|c|}{ Neuron 01 (Route 5) } & \multicolumn{3}{|c|}{ Neuron o2 (Route 8) } \\
\hline & Target & Meas & Diff & Target & Meas & Diff \\
\hline$i 1$ & 0.3708 & 0.35205 & 0.01875 & 0.3838 & 0.33991 & 0.04389 \\
\hline$i 2$ & 0.6027 & 0.5856 & 0.0171 & 0.4619 & 0.45071 & 0.01119 \\
\hline 13 & 0.5031 & 0.48237 & 0.02073 & 0.3623 & 0.35385 & 0.00845 \\
\hline i4 & 0.3088 & 0.24995 & 0.05885 & 0.2418 & 0.23459 & 0.00721 \\
\hline i5 & 0.213 & 0.27724 & -0.0642 & 0.3376 & 0.27887 & 0.05873 \\
\hline 16 & 0.5481 & 0.52539 & 0.02271 & 0.3776 & 0.36714 & 0.01046 \\
\hline$i 7$ & 0.4084 & 0.32837 & 0.08003 & 0.2379 & 0.24195 & -0.0041 \\
\hline i 8 & 0.4225 & 0.28845 & 0.13405 & 0.1281 & 0.21074 & -0.0826 \\
\hline i9 & & 0.0526 & -0.0526 & & 0.03387 & -0.0339 \\
\hline i10 & 0.6212 & 0.65128 & -0.0301 & 0.3761 & 0.37922 & -0.0031 \\
\hline$\lceil 11$ & 0.5181 & 0.54835 & -0.0303 & 0.273 & 0.28818 & -0.0152 \\
\hline$i 12$ & 0.5989 & 0.59535 & 0.00355 & 0.3045 & 0.28943 & 0.01507 \\
\hline$i 13$ & 0.7597 & 0.75393 & 0.00577 & 0.4653 & 0.47533 & -0.01 \\
\hline ¡14 & 0.663 & 0.70045 & -0.0375 & 0.4179 & 0.40757 & 0.01033 \\
\hline i15 & 0.6536 & 0.66736 & -0.0138 & 0.4085 & 0.37255 & 0.03595 \\
\hline i16 & 0.8046 & 0.79197 & 0.01263 & 0.5102 & 0.49962 & 0.01058 \\
\hline \multirow{4}{*}{$\begin{array}{l}\text { Neuron } \\
\text { Results }\end{array}$} & \multicolumn{2}{|c|}{ Average [Diff| (Full) } & 0.03766 & \multicolumn{2}{|c|}{ Average |Diff (Full) } & 0.02255 \\
\hline & \multicolumn{2}{|c|}{ Average $\mid$ Diff $\mid$ (Mod) } & 0.03666 & \multicolumn{2}{|c|}{ Average |Diff (Mod) } & 0.02179 \\
\hline & \multicolumn{2}{|c|}{ Std Dev |Diff] (Full) } & 0.03391 & \multicolumn{2}{|c|}{ Std Dev |Diff (Full) } & 0.02262 \\
\hline & \multicolumn{2}{|c|}{ Std Dev |Diff| (Mod) } & 0.03485 & \multicolumn{2}{|c|}{ Std Dev |Diff| (Mod) } & 0.0232 \\
\hline
\end{tabular}

\begin{tabular}{|c|c|c|c|c|c|c|}
\hline \multirow[t]{2}{*}{ Destination } & \multicolumn{3}{|c|}{ Neuron o3 (Route 12) } & \multicolumn{3}{|c|}{ Neuron o4 (Route 13) } \\
\hline & Target & Meas & Diff & Target & Meas & Diff \\
\hline$i 1$ & 0.5 & 0.61316 & -0.0147 & 0.8306 & 0.84129 & 0.010 \\
\hline $\mathrm{i} 2$ & 0.6191 & 0.62066 & -0.0016 & 0.8512 & 0.84059 & 0.01061 \\
\hline i 3 & 0.5195 & 0.50933 & 0.01017 & 0.7516 & 0.75722 & -0.0056 \\
\hline ¡ 4 & 0.4565 & 0.44457 & 0.01193 & 0.6886 & 0.70087 & -0.0123 \\
\hline i 5 & 0.5 & 0.5 & 0.01624 & 0.7 & 0.78113 & 0.00327 \\
\hline$i 6$ & 0.5072 & 0.49369 & 0.01351 & 0.7 & 0.7 & -0.0031 \\
\hline$i 7$ & 0.395 & 0.38487 & 0.01013 & 0.6271 & 0.65044 & -0.023 \\
\hline i 8 & 0.3428 & 0.33575 & 0.00705 & 0.5749 & 0.58957 & -0.014 \\
\hline i 9 & & 0.03763 & -0.0376 & & 0.0 & -0.0622 \\
\hline i10 & 0.3 & 0.36284 & 0.02 & 0.6 & 0.5 & 0.04445 \\
\hline & 0.28 & 0.2 & 0.02122 & 0.5 & 0.45055 & 6685 \\
\hline & 0 . & 0.2 & -0.0463 & 0.3985 & 0.34866 & 984 \\
\hline & 0.3 & 0.28202 & 0.04518 & 0.2377 & 0.24983 & -0 . \\
\hline$i 14$ & 0.3851 & 0.36627 & 0.01883 & 0.6172 & 0.57499 & 0.04221 \\
\hline$\lcm{115}$ & 0.2762 & 0.30839 & -0.0322 & 0.5083 & 0.47451 & 379 \\
\hline i1 & 0.3712 & 0.34629 & 0.02491 & 0.3929 & 0.4174 & -0.0245 \\
\hline \multirow{4}{*}{$\begin{array}{l}\text { Neuron } \\
\text { Results }\end{array}$} & \multicolumn{2}{|c|}{ Average |Diff| (Full) } & 0.02106 & \multicolumn{2}{|c|}{ Average $\mid$ Diff $\mid$ (Full) } & 0.02622 \\
\hline & \multicolumn{2}{|c|}{\begin{tabular}{|l|l|} 
Average & Diff \\
\end{tabular}} & 0.01996 & \multicolumn{2}{|c|}{ Average |Diff $\mid$ (Mod) } & 0.02382 \\
\hline & \multicolumn{2}{|c|}{\begin{tabular}{|l|l|l|} 
Std Dev & Diff \\
\end{tabular}} & 0.01335 & \multicolumn{2}{|c|}{ Std Dev |Diff | (Full) } & 0.02106 \\
\hline & \multicolumn{2}{|c|}{ Std Dev |Diff| (Mod) } & 0.01304 & \multicolumn{2}{|c|}{ Std Dev [Diff] (Mod) } & 0.01942 \\
\hline
\end{tabular}

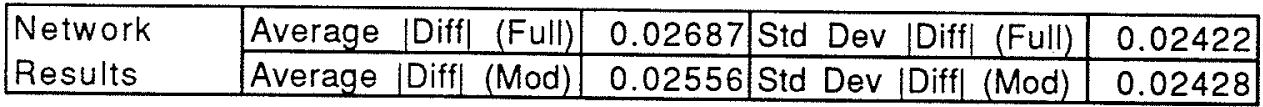


Training Results for V1.2.10.4

(Model 2.06.4, V1 Macro)

\begin{tabular}{|c|c|c|c|c|c|c|}
\hline \multirow[t]{2}{*}{ Destination } & \multicolumn{3}{|c|}{ Neuron o1 (Route 5) } & \multicolumn{3}{|c|}{ Neuron o2 (Route 8) } \\
\hline & Target & Meas & Diff & Target & Meas & Diff \\
\hline$i 1$ & 0.3708 & 0.34781 & 0.02299 & 0.3838 & 0.34264 & 0.04116 \\
\hline$i 2$ & 0.6027 & 0.5936 & 0.0091 & 0.4619 & 0.45209 & 0.00981 \\
\hline i3 & 0.5031 & 0.46029 & 0.04281 & 0.3623 & 0.35066 & 0.01164 \\
\hline ¡ 4 & 0.3088 & 0.28944 & 0.01936 & 0.2418 & 0.24264 & -0.0008 \\
\hline i 5 & 0.213 & 687 & 0.01613 & 0.3 & 0.26826 & 0.06934 \\
\hline 16 & 0.5481 & 75 & 0.0 & 76 & 0.36484 & 0.01276 \\
\hline$i 7$ & 0.4 & 0.3 & 0.05055 & 0.2 & 0.2347 & 0.0032 \\
\hline i 8 & 0.4225 & 0.32037 & 0.10213 & 0.1281 & 0.20368 & -0.0756 \\
\hline i 9 & & 0.0652 & -0.0652 & & 0.04462 & -0.0446 \\
\hline$\$ 10$ & 0.6212 & 0.63596 & -0.0148 & 0.3761 & 0.3 & -0.0004 \\
\hline$\lceil 11$ & 0.5181 & 0.5 & -0.0417 & 0.273 & 0.29702 & -0.024 \\
\hline ¡12 & 0.5989 & 0.59126 & 0.00764 & 0.3045 & 0.30243 & 0.00207 \\
\hline$\lceil 13$ & 0.7597 & 0.7 & 0.01157 & 53 & 53 & -0. \\
\hline$\lceil 14$ & 0.663 & 0.7 & -0. & 79 & 0.41836 & -0.0005 \\
\hline$i 15$ & 0.6536 & 0.6 & -0.0179 & 0.4085 & 0.3817 & 0.0268 \\
\hline$i 16$ & 0.8046 & 0.7784 & 0.0262 & 0.5102 & 0.48441 & 0.02579 \\
\hline \multirow{4}{*}{$\begin{array}{l}\text { Neuron } \\
\text { Results }\end{array}$} & \multicolumn{2}{|c|}{ Average |Diff| (Full) } & 0.03208 & \multicolumn{2}{|c|}{\begin{tabular}{|l|l|} 
Average Diff $\mid$ (Full) \\
\end{tabular}} & 0.0218 \\
\hline & \multicolumn{2}{|c|}{ Average $\mid$ Diff $\mid$ (Mod) } & 0.02987 & \multicolumn{2}{|c|}{ Average |Diff $\mid$ (Mod) } & 0.02027 \\
\hline & \multicolumn{2}{|c|}{ Std Dev $\mid$ Diff $\mid$ (Full) } & 0.02514 & \multicolumn{2}{|c|}{ Std Dev |Diff| (Full) } & 0.02446 \\
\hline & \multicolumn{2}{|c|}{ Std Dev |Diff| (Mod) } & 0.02437 & \multicolumn{2}{|c|}{ Std Dev [Diff] (Mod) } & 0.02452 \\
\hline
\end{tabular}

\begin{tabular}{|c|c|c|c|c|c|c|}
\hline \multirow[t]{2}{*}{ Destination } & \multicolumn{3}{|c|}{ Neuron o3 (Route 12) } & \multicolumn{3}{|c|}{ Neuron o4 (Route 13) } \\
\hline & Target & Meas & Diff & Target & Meas & Diff \\
\hline$i 1$ & 0.5985 & 0.61117 & -0.0127 & 0.8306 & 0.84178 & -0.0112 \\
\hline i2 & 0.6191 & 0.61465 & 0.00445 & 0.8512 & 0.83766 & 0.01354 \\
\hline i3 & 0.5195 & 0.50773 & 0.01177 & 0.7516 & 0.75736 & -0.0050 \\
\hline ¡4 & 0.4565 & 0.42852 & 0.02798 & 0.6886 & 0.69339 & -0.0048 \\
\hline i5 & 0.5523 & 0.56724 & -0.0149 & 0.7 & 0.797 & -0.0126 \\
\hline i 6 & 0.50 & 0.4 & 0.01197 & 0.7393 & 0.74773 & -0.0084 \\
\hline i 7 & 0.3 & 0.37192 & 0.02308 & 0.6271 & 0.65121 & -0.0241 \\
\hline i8 & 0.3428 & 0.32307 & 0.01973 & 0.5749 & 0.59463 & -0.0197 \\
\hline 19 & & 0.05946 & -0.0595 & & 0.10837 & -0.1084 \\
\hline$i 10$ & 0.3884 & 0.37162 & 0.01678 & 0.6205 & 0.57796 & 0.04254 \\
\hline$i 11$ & 0.2853 & 0.25649 & 0.02881 & 0.5174 & 0.40723 & 017 \\
\hline$i 12$ & 0.1664 & 0.21906 & .0 .0527 & 0.3985 & 292 & 0.07558 \\
\hline$i 13$ & 0.32 & 0.2 & 0.05 & 0.2 & 0.2 & -0.0307 \\
\hline$i 14$ & 0.3 & 0.3 & 0.02349 & 0.6172 & 0.5429 & 0.0743 \\
\hline$i 15$ & 0.2762 & 0.30684 & -0.0306 & 0.5083 & 0.45363 & 0.05467 \\
\hline$\$ 16$ & 0.3712 & 0.34605 & 0.02515 & 0.3929 & 0.45789 & -0.065 \\
\hline \multirow{4}{*}{$\begin{array}{l}\text { Neuron } \\
\text { Results }\end{array}$} & \multicolumn{2}{|c|}{ Average |Diff $\mid$ (Full) } & 0.02637 & \multicolumn{2}{|c|}{ Average |Diff (Full) } & 0.04134 \\
\hline & \multicolumn{2}{|c|}{ Average $\mid$ Diff $\mid$ (Mod) } & 0.02416 & \multicolumn{2}{|c|}{ Average [Diff| (Mod) } & 0.03687 \\
\hline & \multicolumn{2}{|c|}{ Std Dev |Diff $\mid$ (Full) } & 0.01675 & \multicolumn{2}{|c|}{$\begin{array}{l}\text { Std Dev |Diff } \mid \text { (Full) } \\
\end{array}$} & 0.03587 \\
\hline & \multicolumn{2}{|c|}{ Std Dev |Diff| (Mod) } & 0.01473 & \multicolumn{2}{|c|}{ Std Dev |Diff| (Mod) } & 0.03219 \\
\hline
\end{tabular}

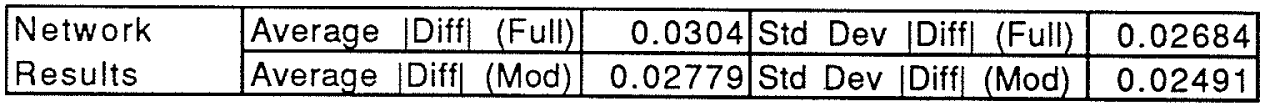


Training Results for V1.2.06.6

(Network 2.06.6, V1 Macro)

\begin{tabular}{|c|c|c|c|c|c|c|c|c|c|}
\hline \multirow[t]{2}{*}{ Destination } & \multicolumn{3}{|c|}{ Neuron o1 (Route 7) } & \multicolumn{3}{|c|}{ Neuron o2 (Route 8) } & \multicolumn{3}{|c|}{ Neuron o3 (Route 10) } \\
\hline & Target & Meas & Diff & Target & Meas & Diff & Target & Meas & Diff \\
\hline$i 1$ & 0.3763 & .32439 & 0.05191 & 0.4256 & 0.45136 & -0.0258 & 0.4882 & 0.446 & 0.042 \\
\hline 12 & 0.3588 & 0.35 & 0.00 & 0.5037 & 0.50846 & -0.0048 & 0.3793 & 0.35668 & 0.0226 \\
\hline$i 3$ & & 0.26 & -0.0018 & $0.4-2-3-3-1$ & 0.3439 & 0.0602 & 559 & .40491 & \\
\hline$\lceil 4$ & 0.2 & 0.19 & 0.03587 & 0.2 & 0.25 & 0.02455 & 0.4862 & 0.4399 & \\
\hline i 5 & 0.3301 & 0.32423 & 0.00 & 0.3 & 0.3 & 0.036 & 0.582 & 37 & -0 \\
\hline$i 6$ & 0.2745 & 0.29 & -0.0244 & 0.4 & 0.4 & -0.0262 & 0.247 & 24 & 0.0 \\
\hline$i 7$ & 0.1348 & 0.165 & -0.0311 & 0.2 & & 0.0 & & & -0.007 \\
\hline i 8 & 0.2445 & 0.24108 & 0.00342 & 0.1 & 0.2 & -0 & & & -0.015 \\
\hline i9 & 0.3476 & 0.30476 & 0.04284 & 0. & 0.2 & -0 . & & 0.53 & \\
\hline$i 10$ & 0.3933 & 0.3 & 0.0 & 0.5 & 0.5 & 0.0 & 0.1281 & & \\
\hline$i 11$ & & 0.0 & -0.0 & & 0.02386 & -0.0239 & & 0.0 & -0.016 \\
\hline$i$ & 0.4 & 0.42 & -0.0013 & 0.3 & 0.35 & -0 . & 10 & & 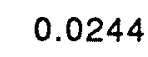 \\
\hline$i 13$ & 0.5603 & 0.56079 & -0.0005 & 0.4857 & 0.4 & 0.0 & 321 & 0.6 & -0. \\
\hline$\lceil 14$ & 0.4815 & 0.50309 & -0.0216 & 0.6331 & 0.5 & 0.0 & 26 & 0.2 & -0.010 \\
\hline$i 15$ & 0.5307 & 0.52237 & 0.00833 & 0.456 & 0.4 & -0. & 0.3615 & 0.36893 & -0.007 \\
\hline$i 1$ & 0.6266 & 0.63594 & -0.0093 & 0.552 & 0.60312 & -0.0511 & 0.5418 & 0.50516 & 0.0366 \\
\hline \multirow{4}{*}{$\begin{array}{l}\text { Neuron } \\
\text { Results }\end{array}$} & \multicolumn{2}{|c|}{ Average |Diff (Fuli) } & 0.0185 & \multicolumn{2}{|c|}{ Average $\mid$ Diff $\mid$ (Full) } & 0.03239 & \multicolumn{2}{|c|}{ Average |Diff| (Fuli) } & \\
\hline & \multicolumn{2}{|c|}{ Average |Diff (Mod) } & 0.0183 & \multicolumn{2}{|c|}{ Average |Diff (Mod) } & 0.03296 & \multicolumn{2}{|c|}{\begin{tabular}{|l|l|l|} 
Average & Diff & (Mod) \\
\end{tabular}} & \\
\hline & \multicolumn{2}{|c|}{ Std Dev |Diff (Full) } & 0.01634 & \multicolumn{2}{|c|}{ Std Dev $\mid$ Diff $\mid$ (Full) } & 0.0 & \multicolumn{2}{|c|}{ Std Dev |Diff| (Full) } & 0.0198 \\
\hline & \multicolumn{2}{|c|}{ Std Dev [Diff (Mod) } & 0.01689 & \multicolumn{2}{|c|}{ Std Dev |Diff $\mid$ (Mod) } & 0.02337 & \multicolumn{2}{|c|}{ Std Dev $\mid$ Diff $\mid$ (Mod) } & 0.0202 \\
\hline
\end{tabular}

\begin{tabular}{|c|c|c|c|c|c|c|c|c|c|}
\hline \multirow[t]{2}{*}{ Destination } & \multicolumn{3}{|c|}{ Neuron o4 (Route 12) } & \multicolumn{3}{|c|}{ Neuron o5 (Route 14) } & \multicolumn{3}{|c|}{ Neuron o6 (Route 15) } \\
\hline & Tarc & Meas & Diff & Target & Meas & Diff & Target & Meas & Diff \\
\hline$i 1$ & 0.5759 & 0.6301 & .0 .0542 & 0.6182 & 60604 & 0.01216 & 0.7023 & 0.67104 & 0.03126 \\
\hline$i 2$ & 0.6541 & 0.63262 & 2148 & 0.5092 & 0.50591 & 0.00329 & 0.6088 & 0.61179 & -0.00 \\
\hline i3 & 0.5545 & 891 & 559 & 0.4859 & 0.52778 & -0.0419 & 54 & 0.5 & 0.0297 \\
\hline i 4 & $0 . c^{2}$ & 6464 & -0.0307 & 0.6162 & 0.58 & 0.03103 & & & -0.03 \\
\hline$i 5$ & 0.4 & 0.464 & 0.0092 & 0.712 & 0.6 & 0.0376 & 97 & 21 & -0.014 \\
\hline$i 6$ & 0.5696 & 0.529 & 0.0406 & 0.3 & 0.3 & 0.02437 & 65 & 097 & 0. \\
\hline$i 7$ & 0.43 & 0.40619 & 0.02381 & 0.5166 & 0.53532 & -0 . & 64 & 716 & 0.0192 \\
\hline i 8 & 0.3203 & 0.2 & 0.04366 & 0.565 & 0.5 & 0.0 & 67 & 0. & 0. \\
\hline i9 & 0.2853 & 0.3 & -0.0213 & 0.53 & & 16 & & & -0.027 \\
\hline$i 10$ & 0.4871 & 0.5 & -0.0163 & 0.2944 & 0.2 & 0.03073 & 0.3939 & 0. & 0. \\
\hline$i 11$ & & 0.0 & -0.0 & & 0.0 & -0.0161 & & & \\
\hline$i 12$ & 39 & 47 & -0.0608 & 0.3886 & 0.3 & 0.02697 & 0.2703 & 301 & \\
\hline$i 13$ & 0.3047 & & 0.0222 & 0.5494 & 0.5 & -0 . & 0.4311 & 925 & 185 \\
\hline$i 14$ & 0.3626 & 0.34563 & 0.01697 & 0.1699 & 0.2 & -0 . & 95 & 0.2 & 0.0113 \\
\hline$i 15$ & 0.2536 & 0.1 & 0.0 & 0.2789 & 0.2 & 0.0 & 0.1605 & 0.1837 & -0.023 \\
\hline$i$ & 0.3496 & 0.34465 & 0.0 & 0.4591 & 0.41313 & 0.0 & 0.3408 & 0.32935 & 0.0 \\
\hline \multirow{4}{*}{$\begin{array}{l}\text { Neuron } \\
\text { Results }\end{array}$} & \multicolumn{2}{|c|}{ Average |Diff (Full) } & 0.03174 & \multicolumn{2}{|c|}{ Average |Diff| (Full) } & 0.02659 & \multicolumn{2}{|c|}{ Average |Diff | (Full) } & \\
\hline & \multicolumn{2}{|c|}{ Average |Diff| (Mod) } & 0.03254 & \multicolumn{2}{|c|}{ Average [Diff] (Mod) } & 0.02729 & \multicolumn{2}{|c|}{\begin{tabular}{|l|l|} 
Average & Diff \\
(Mod)
\end{tabular}} & \\
\hline & \multicolumn{2}{|c|}{ Std Dev |Diff| (Full) } & 0.01918 & \multicolumn{2}{|c|}{ Std Dev |Diff| (Full) } & 0.0 & \multicolumn{2}{|c|}{ Std Dev |Diff| (Full) } & 0.01221 \\
\hline & \multicolumn{2}{|c|}{ IStd Dev |Diff $\mid$ (Mod) } & 0.01957 & \multicolumn{2}{|c|}{ Std Dev |Diff| (Mod) } & 0.01718 & \multicolumn{2}{|c|}{ Std Dev Diff $\mid$ (Mod) } & 0.01261 \\
\hline
\end{tabular}

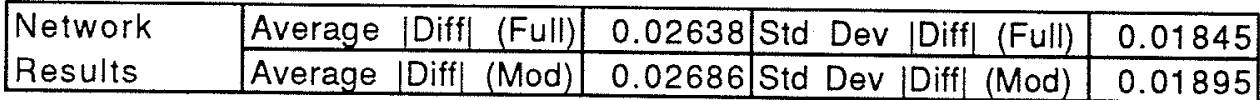


Training Results for V1.2.08.6

(Netork 2.08.6, V1 Macro)

\begin{tabular}{|c|c|c|c|c|c|c|c|c|c|}
\hline \multirow[t]{2}{*}{ Destination } & \multicolumn{3}{|c|}{ Neurón o1 (Route 7) } & \multicolumn{3}{|c|}{ Neuron o2 (Route 8) } & \multicolumn{3}{|c|}{ Neuron o3 (Route 10) } \\
\hline & Target & Meas & Diff & Target & Theas & Diו & Target & Meas & Diff \\
\hline$i 1$ & 0.3763 & 0.3393 & 0.037 & 0.4256 & 0.44591 & -0.0203 & 0.4882 & 0.47327 & 0.014 \\
\hline$i 2$ & 0.3588 & 0.33533 & .02347 & 0.5037 & 0.50079 & 0.00291 & 0.3793 & 0.34106 & .0382 \\
\hline$i 3$ & 0.2 & 0.27336 & -0.0142 & 0.4041 & 0.39986 & 0.00424 & 59 & 25 & 0.0316 \\
\hline ¡ 4 & 0.2 & 0.21966 & 0.01464 & 0.2836 & 0.2 & 0.0 & 0.4862 & 54 & 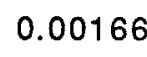 \\
\hline i5 & 0.3301 & 0.3674 & -0.0373 & 0.3 & 0.37739 & 0.00201 & 0.582 & 385 & -0.011 \\
\hline i 6 & 0.2745 & 0.25682 & 0.01768 & 0.4194 & 0.3 & 0.02016 & 0.247 & 45 & 0.0025 \\
\hline i7 & 0.1348 & 0.16452 & -0.0297 & 0.2797 & 0.2 & 0.0 & & & -0.010 \\
\hline i 8 & 0.2445 & 0.21343 & 0.03107 & 0.1 & 0.2 & -0 & & & -0.006 \\
\hline i9 & 0.3476 & 0.32686 & 0.02074 & 0.2 & 0.2 & -0.0 & & & 0.0 \\
\hline$i 10$ & 0.3933 & 0.34171 & 0.05159 & 0.5382 & 0 . & 0.017 & 0.1281 & 0.1 & -0. \\
\hline$i 11$ & & 0.0 & -0.0212 & & 0.0 & -0.0239 & & 0.0 & -0 \\
\hline$i 12$ & & 0.4 & -0.0076 & 0.3463 & 0.32723 & 0.01907 & 0. & 26 & \\
\hline $\mathrm{i}$ & 0.5603 & 0.53861 & 0.02169 & 0.4857 & 0.46 & 0.02028 & 0.6321 & 966 & 0.0 \\
\hline$i 14$ & 0.4815 & 0.50891 & -0.0274 & 0.6331 & 0.65 & -0.0178 & 0.2526 & 0.17443 & 0.07817 \\
\hline$i 15$ & 0.5307 & 0.53985 & -0.0092 & 0.456 & 0.4 & 0.00609 & 0.3615 & 0.37604 & -0.014 \\
\hline$i 1$ & 0.6266 & 0.61148 & 0.01512 & 0.552 & 0.53611 & 0.01589 & 0.5418 & 0.55379 & -0.01 \\
\hline \multirow{4}{*}{$\begin{array}{l}\text { Neuron } \\
\text { Results }\end{array}$} & \multicolumn{2}{|c|}{ Average [Diff| (Full) } & 0.02372 & \multicolumn{2}{|c|}{ Average |Diff| (Full) } & 0.01899 & \multicolumn{2}{|c|}{ Average [Diff (Full) } & 0.0224 \\
\hline & \multicolumn{2}{|c|}{ Average $\mid$ Diff $\mid$ (Mod) } & 0.02389 & \multicolumn{2}{|c|}{ Average |Diff| (Mod) } & 0.01866 & \multicolumn{2}{|c|}{\begin{tabular}{l|l|l|} 
Average & Diff & (Mod) \\
\end{tabular}} & \\
\hline & \multirow{2}{*}{\multicolumn{2}{|c|}{ Std Dev |Diff | (Full) }} & 0.01159 & \multicolumn{2}{|c|}{ Std Dev [Diff| (Full) } & 0.01638 & \multicolumn{2}{|c|}{ Std Dev |Diff $\mid$ (Full) } & \\
\hline & & Std Dev $\mid$ Diff $\mid$ (Mod) & 0.01198 & \multicolumn{2}{|c|}{ Std Dev |Diff| (Mod) } & 0.0169 & \multicolumn{2}{|c|}{ Std Dev |Diff| (Mod) } & 0.0226 \\
\hline
\end{tabular}

\begin{tabular}{|c|c|c|c|c|c|c|c|c|c|}
\hline \multirow[t]{2}{*}{ Destination } & \multicolumn{3}{|c|}{ Neuron o4 (Route 12) } & \multicolumn{3}{|c|}{ Neuron o5 (Route 14) } & \multicolumn{3}{|c|}{ Neuron o6 (Route 15) } \\
\hline & Target & Meas & Diff & Target & Meas & Diff & Tar & Meas & Diff \\
\hline i1 & 0.5759 & 0.60478 & -0.0289 & 0.6182 & 0.61441 & 0.00379 & 0.7023 & 0.65797 & 0.0443 \\
\hline i 2 & 0.6541 & 0.64947 & 0.00463 & 0.5092 & 0.50728 & 0.00192 & 0.6088 & 0.62994 & -0.021 \\
\hline$i 3$ & 0.5545 & 0.56139 & -0.0069 & 0.4859 & 0.47805 & 0.00785 & 0.5854 & 0.57125 & 0.01415 \\
\hline i 4 & 0.4339 & 0.42594 & 0.00796 & 0.6162 & 0.59929 & 0.01691 & & 0.5 & -0.0074 \\
\hline i5 & 0.4732 & 0.4597 & 0.0135 & 0.712 & 0.6 & 0.05722 & & 0. & \\
\hline i6 & 0.5696 & 0.53622 & 0.03338 & 0.3 & 0.3 & -0.0059 & & & 29 \\
\hline i 7 & 0.43 & 0.40664 & 0.02336 & 0.5166 & 0.5 & -0.022 & & 0.53 & 03 \\
\hline i 8 & 0.3203 & 0.28837 & 0.03193 & 0.565 & 0. & 0.0137 & 0.4 & 35 & -0 \\
\hline i9 & 0.2853 & 0.27248 & 0.01282 & 0.53 & 0.5 & -0.0242 & 0. & & 0. \\
\hline i10 & 0.4871 & 0.50188 & -0.0148 & 0.2944 & 0.2 & 0.03379 & 0.3939 & 0.3 & -0.0025 \\
\hline$i 11$ & & 0.02287 & -0.0229 & & 0.0 & 191 & & & -0.021 \\
\hline$i 12$ & .1439 & 0.17667 & -0.0328 & 0.3 & 0.3 & 669 & & & \\
\hline & 0.3047 & 0.3 & -0.0277 & 0.5 & 0.5 & -0.0102 & 0.4 & & 12 \\
\hline & 0.3626 & 0.35 & 0.00415 & 0.1699 & 0.14863 & 0.02127 & 0. & & 0. \\
\hline & 0.2536 & 0.16761 & 0.08599 & 0.2789 & 0.2 & 0.0 & 0. & & 0.00114 \\
\hline & 0.3496 & 0.30618 & 0.04342 & 0.4591 & 0.46552 & -0. & 0.3408 & 0.34009 & 0.00071 \\
\hline \multirow{4}{*}{$\begin{array}{l}\text { Neuron } \\
\text { Results }\end{array}$} & \multicolumn{2}{|c|}{ Average |Diff| (Full) } & 0.02469 & \multicolumn{2}{|c|}{ Average [Diff (Full) } & 0.01824 & \multicolumn{2}{|c|}{ Average |Diff (Full) } & 0.01749 \\
\hline & \multicolumn{2}{|c|}{ Average |Diff| (Mod) } & 0.02481 & \multicolumn{2}{|c|}{ Average JDiff (Mod) } & 0.01818 & \multicolumn{2}{|c|}{ Average |Diff| (Mod) } & 1725 \\
\hline & \multicolumn{2}{|c|}{\begin{tabular}{|l|l|} 
Std Dev $\mid$ Diff $\mid$ (Full) \\
\end{tabular}} & 0.0202 & \multicolumn{2}{|c|}{ Std Dev [Diff] (Full) } & 0.01401 & \multicolumn{2}{|c|}{ Std Dev |Diff| (Full) } & \\
\hline & \multicolumn{2}{|c|}{ Std Dev |Diff| (Mod) } & 0.0209 & \multicolumn{2}{|c|}{ Std Dev |Diff| (Mod) } & 0.0145 & \multicolumn{2}{|c|}{ Std Dev IDiff / (Mod) } & 0.01723 \\
\hline
\end{tabular}

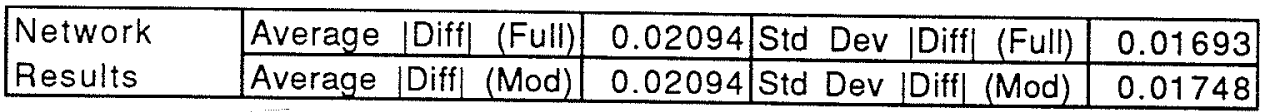


Training Results for V1.2.10.6

(Network 2.10.6, V1 Macro)

\begin{tabular}{|c|c|c|c|c|c|c|c|c|c|}
\hline \multirow[t]{2}{*}{ Destination } & \multicolumn{3}{|c|}{ Neuron o1 (Route 7) } & \multicolumn{3}{|c|}{ Neuron o2 (Route 8) } & \multicolumn{3}{|c|}{ Neuron 03 (Route 10) } \\
\hline & Target & Meas & Diff & Target & Meas & Diff & Target & Meas & Diff \\
\hline$i 1$ & 0.3763 & 0.34288 & 0.03342 & 0.4256 & 0.45625 & -0.0307 & 0.4882 & .46024 & .027 \\
\hline$i 2$ & 0.3588 & 0.33848 & 0.02032 & 0.5037 & 0.50784 & -0.0041 & 0.3793 & 0.32407 & 0.055 \\
\hline$i 3$ & 0.2592 & 0.2 & -0.0118 & 0.4041 & 0.4008 & 0.0033 & 0.3559 & 0.31452 & 0.0415 \\
\hline ¡4 & 0.2 & 0.2 & 0.02576 & 0.2836 & 0.25214 & 0.03146 & 362 & 0.4 & -0.003 \\
\hline i 5 & 0. & 0.3 & -0.0254 & 0.3794 & 0.35879 & 0.02061 & 0.582 & 69 & -0.042 \\
\hline 16 & 0.2 & 0.2 & 0.0169 & 0.4 & 0.39233 & 0.02707 & 0.247 & 07 & -0.004 \\
\hline$i 7$ & 0.1348 & 0.13883 & -0.004 & 0.2797 & 0.19369 & 0.08601 & 67 & & -0 \\
\hline i8 & 0.2445 & 0.17983 & 0.06467 & 0.1699 & 864 & -0.0187 & 0.4964 & 43 & \\
\hline i 9 & 0.3476 & 0.31847 & 0.02913 & 0.273 & 0.28456 & -0.0116 & 0.5995 & 46 & 0.0 \\
\hline ¡10 & 0.3933 & 0.37848 & 0.01482 & 0.5382 & 0.52774 & 0.01046 & 0.1281 & 0.21 & -0.084 \\
\hline ¡11 & & 0.02429 & -0.0243 & & 0.0 & 64 & & 0.0 & -0.024 \\
\hline 2 & & 0.4 & -0.0 & 0.3 & 0.3 & 706 & 0.4713 & 0.4 & -0.010 \\
\hline & & & 0.0141 & 0.4857 & 0.46326 & 0.02244 & 0.6321 & 0.62 & 0.0075 \\
\hline & 0.4815 & 0.49425 & -0.0128 & 0.6331 & 0.64468 & -0.0116 & 0.2526 & 0.1 & 0.1 \\
\hline & 0.5307 & 0.51893 & 0.01177 & 0.456 & 0.45861 & -0.0 & 15 & 0. & $-1 E-0$ \\
\hline$i$ & 0.6266 & 0.62673 & -0.0001 & 0.552 & 0.55495 & -0.003 & 0.5418 & 0.54498 & -0.003 \\
\hline \multirow{4}{*}{$\begin{array}{l}\text { Neuron } \\
\text { Results }\end{array}$} & \multicolumn{2}{|c|}{ Average |Diff| (Full) } & 0.02162 & \multicolumn{2}{|c|}{ Average Diff] (Full) } & 0.02106 & \multicolumn{2}{|c|}{ Average (Diff (Full) } & 0.0278 \\
\hline & \multicolumn{2}{|c|}{ Average |Diff| (Mod) } & 0.02145 & \multicolumn{2}{|c|}{ Average |Diff $\mid$ (Mod) } & 0.02071 & \multicolumn{2}{|c|}{ Average Diff (Mod) } & 0.0280 \\
\hline & \multicolumn{2}{|c|}{ Std Dev |Diff| (Full) } & 0.01524 & \multicolumn{2}{|c|}{ Std Dev |Diff| (Full) } & 0.02015 & \multicolumn{2}{|c|}{ Std Dev |Diff| (Full) } & 0.0293 \\
\hline & Std Dev 15 & iff $($ Mod) & 0.01576 & Std Dev 10 & iff: (Mod) & 0.0208 & Std Dev $1 D$ & iff $\mid$ (Mod) & 0.0303 \\
\hline
\end{tabular}

\begin{tabular}{|c|c|c|c|c|c|c|c|c|c|}
\hline \multirow[t]{2}{*}{ Destination } & \multicolumn{3}{|c|}{ Neuron o4 (Route 12) } & \multicolumn{3}{|c|}{ Neuron o5 (Route 14) } & \multicolumn{3}{|c|}{ Neuron o6 (Route 15) } \\
\hline & Tar & Meas & Diff & Target & Meas & Diff & Target & Meas & Diff \\
\hline i 1 & 0.5759 & 0.61843 & -0.0425 & 82 & 79 & 0.01441 & 0.7023 & 0.6563 & 0.04 \\
\hline$i 2$ & 0.6541 & 0.66501 & -0.0109 & 92 & 14 & 0.01806 & 0.6088 & 0.63166 & -0.022 \\
\hline 13 & 0.5545 & 336 & 89 & 0.4 & 59 & 0.01831 & 0.5854 & 0.57252 & 0.0128 \\
\hline i 4 & 39 & 75 & 0.0 & 0.6 & 34 & 0.01136 & 0. & 0. & -0.005 \\
\hline i5 & 32 & 0.4 & 694 & 12 & 62 & 0.0 & 97 & & 0.0168 \\
\hline 16 & 0.5696 & 0.5 & 241 & & & -0. & & & \\
\hline i 7 & 0.43 & 0.41 & 0.01267 & 0.5 & & & 64 & 0. & 0.0710 \\
\hline i 8 & 0.3203 & 0.29412 & 0.0 & 0.565 & 6 & 0.00994 & 67 & 56 & 009 \\
\hline$i 9$ & 0.2853 & 0.28519 & 0.0 & 0.53 & 43 & -0.0244 & 0.4 & 11 & -0.005 \\
\hline i10 & 0.4871 & 0.47372 & 0.01338 & 0.2944 & 0.2 & 0.0 & 0.3939 & 0.37715 & 0.01675 \\
\hline$i 11$ & & 0.02698 & -0 & & 0.0 & -0 . & & 0.02743 & -0.027 \\
\hline$i 1$ & & 0.1 & -0 . & & 36 & 0.02774 & & & 0. \\
\hline$i 1$ & 0.3047 & 0.3 & -0.0065 & 0.5 & 0.5 & -0 & 0.4 & & 0.0228 \\
\hline$\lceil 14$ & 0.3626 & 0.37839 & -0.0158 & 0.1 & 0.1 & 0.0 & 0.2695 & 0.2 & 0.03594 \\
\hline ¡15 & 0.2536 & 0.20 & 0.0 & 0.2789 & 0. & 0. & 0.1605 & & -0.0362 \\
\hline $\mathrm{i1}$ & 0.3496 & 0.30118 & 0.0 & 0.4591 & 0.45127 & & 0.3408 & 0.33281 & 0.00799 \\
\hline \multirow{4}{*}{$\begin{array}{l}\text { Neuron } \\
\text { Results }\end{array}$} & \multicolumn{2}{|c|}{ Average |Diff| (Full) } & 0.02186 & \multicolumn{2}{|c|}{ Average [Diff] (Full) } & 0.01931 & \multicolumn{2}{|c|}{\begin{tabular}{|l|l|} 
Average & Diff \\
\end{tabular}} & 0.024 \\
\hline & \multicolumn{2}{|c|}{ Average |Diff| (Mod) } & 0.02152 & \multicolumn{2}{|c|}{ Average |Diff| (Mod) } & 0.0188 & \multicolumn{2}{|c|}{ Average |Diff| (Mod) } & 0.02377 \\
\hline & \multicolumn{2}{|c|}{ Std Dev |Diff (Full) } & 0.01658 & \multicolumn{2}{|c|}{ Std Dev $\mid$ Diff $\mid$ (Full) } & 0.00781 & \multicolumn{2}{|c|}{ Std Dev [Diff $\mid$ (Full) } & \\
\hline & \multicolumn{2}{|c|}{ Std Dev Diff| (Mod) } & 0.0171 & \multicolumn{2}{|c|}{ Std Dev [Diff] (Mod) } & 0.0078 & \multicolumn{2}{|c|}{ Std Dev [Diff] (Mod) } & 0.01865 \\
\hline
\end{tabular}

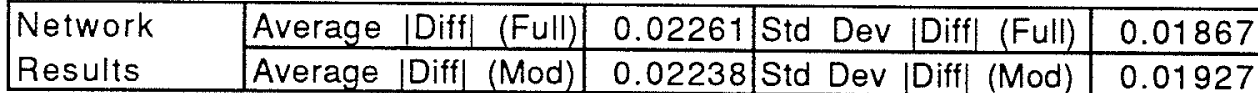


Training Results for V2.2.06.2

(Network 2.06.2, V2 Macro)

\begin{tabular}{|c|c|c|c|c|c|c|}
\hline \multirow[t]{2}{*}{ Destination } & \multicolumn{3}{|c|}{ Neuron o1 (Route 3) } & \multicolumn{3}{|c|}{ Neuron 02 (Route 6) } \\
\hline & Target & Meas & Diff & Target & Meas & Diff \\
\hline i1 & 0.2569 & 0.31309 & -0.0562 & 0.3986 & 0.34103 & 0.05757 \\
\hline i 2 & & 0.34317 & -0.3432 & & 0.3692 & -0.3692 \\
\hline i 3 & 0.1245 & 0.18544 & -0.0609 & 0.2663 & 0.21066 & 0.05564 \\
\hline i 4 & 0.3486 & 0.35828 & -0.0097 & 0.3966 & 0.38638 & 0.01022 \\
\hline i5 & 0.4146 & 0.44063 & -0.026 & 0.4924 & 0.46424 & 0.02816 \\
\hline i 6 & 0.2335 & 0.18121 & 0.05229 & 0.1573 & 0.20552 & -0.0482 \\
\hline i 7 & 0.249 & 0.25843 & -0.0094 & 0.297 & 0.28785 & 0.00915 \\
\hline i 8 & 0.3588 & 0.36858 & -0.0098 & 0.4068 & 0.39643 & 0.01037 \\
\hline i9 & 0.4618 & 0.47515 & -0.0134 & 0.5097 & 0.4953 & 0.0144 \\
\hline i10 & 0.3524 & 0.29949 & 0.05291 & 0.2762 & 0.32862 & -0.0524 \\
\hline i11 & 0.3588 & 0.35477 & 0.00403 & 0.3793 & 0.3831 & -0.0038 \\
\hline$i 12$ & 0.4776 & 0.47591 & 0.00169 & 0.4981 & 0.49988 & -0.0018 \\
\hline i13 & 0.6385 & 0.64429 & -0.0058 & 0.659 & 0.65273 & 0.00627 \\
\hline$i 14$ & 0.4405 & 0.39559 & 0.04491 & 0.3643 & 0.41452 & -0.0502 \\
\hline$i 15$ & 0.4942 & 0.47607 & 0.01813 & 0.4733 & 0.49308 & -0.0198 \\
\hline$i 16$ & 0.6745 & 0.66514 & 0.00936 & 0.6536 & 0.66411 & -0.0105 \\
\hline \multirow{4}{*}{$\begin{array}{l}\text { Neuron } \\
\text { Results }\end{array}$} & \multicolumn{2}{|c|}{ Average |Diff| (Full) } & 0.04486 & \multicolumn{2}{|c|}{ Average |Diff| (Full) } & 0.04673 \\
\hline & \multicolumn{2}{|c|}{ Average |Diff| (Mod) } & 0.02497 & \multicolumn{2}{|c|}{ Average $\mid$ Diff $\mid$ (Mod) } & 0.02523 \\
\hline & \multicolumn{2}{|c|}{ Std Dev |Diff| (Full) } & 0.0823 & \multicolumn{2}{|c|}{ Std Dev |Diff $\mid$ (Full) } & 0.0884 \\
\hline & \multicolumn{2}{|c|}{ Std Dev |Diff| (Mod) } & 0.02183 & \multicolumn{2}{|c|}{ Std Dev $\mid$ Diff $\mid$ (Mod) } & 0.02123 \\
\hline \multirow{2}{*}{$\begin{array}{l}\text { Network } \\
\text { Results }\end{array}$} & \multirow{2}{*}{\multicolumn{2}{|c|}{ 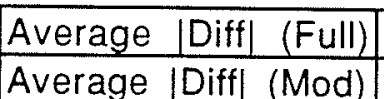 }} & 0.04579 & \multirow{2}{*}{\multicolumn{2}{|c|}{$\begin{array}{l}\text { Std Dev |Diff } \mid \text { (Full) } \\
\text { Std Dev |Diff } \mid \text { (Mod) }\end{array}$}} & 0.08402 \\
\hline & & & 0.0251 & & & 0.02116 \\
\hline
\end{tabular}


Training Results for V2.2.08.2

(Network 2.08.2, V2 Macro)

\begin{tabular}{|c|c|c|c|c|c|c|}
\hline \multirow[t]{2}{*}{ Destination } & \multicolumn{3}{|c|}{ Neuron of (Route 3) } & \multicolumn{3}{|c|}{ Neuron o2 (Route 6) } \\
\hline & Target & Meas & Diff & Target & Meas & Diff \\
\hline$i 1$ & 0.2569 & 0.31318 & -0.0563 & 0.3986 & 0.34232 & 0.05628 \\
\hline i 2 & & 0.34503 & -0.345 & & 0.37134 & -0.3713 \\
\hline$i 3$ & 0.1245 & 0.18331 & -0.0588 & 0.2663 & 0.21173 & 0.05457 \\
\hline i 4 & 0.3486 & 0.3582 & -0.0096 & 0.3966 & 0.38664 & 0.00996 \\
\hline i5 & 0.4146 & 0.44087 & -0.0263 & 0.4924 & 0.4644 & 0.028 \\
\hline i 6 & 0.2335 & 0.18114 & 0.05236 & 0.1573 & 0.20675 & -0.0495 \\
\hline i 7 & 0.249 & 0.25793 & -0.0089 & 0.297 & 0.28839 & 0.00861 \\
\hline i 8 & 0.3588 & 0.36955 & -0.0108 & 0.4068 & 0.39543 & 0.01137 \\
\hline i9 & 0.4618 & 0.47485 & -0.0131 & 0.5 & 0.49584 & 0.01386 \\
\hline i10 & 0.3524 & 0.30073 & 0.05167 & 0.2762 & 0.32784 & -0.0516 \\
\hline$i 11$ & 0.3588 & 0.35572 & 0.00308 & 0.3793 & 0.38211 & -0.0028 \\
\hline i12 & 0.4776 & 0.47731 & 0.00029 & 0.4981 & 0.49838 & -0.0003 \\
\hline i13 & 0.6385 & 0.64395 & -0.0055 & 0.659 & 0.65322 & 0.00578 \\
\hline$i 14$ & 0.4405 & 0.39338 & 0.04712 & 0.3643 & 0.41445 & -0.0502 \\
\hline$i 15$ & 0.4942 & 0.47578 & 0.01842 & 0.4733 & 0.49328 & -0.02 \\
\hline$i 16$ & 0.6745 & 0.66512 & 0.00938 & 0.6536 & 0.664 & -0.0104 \\
\hline \multirow{4}{*}{$\begin{array}{l}\text { Neuron } \\
\text { Results }\end{array}$} & \multicolumn{2}{|c|}{ Average |Diff| (Full) } & 0.04478 & \multicolumn{2}{|c|}{ Average |Diff| (Full) } & 0.04653 \\
\hline & \multicolumn{2}{|c|}{ Average |Diff (Mod) } & 0.02476 & \multicolumn{2}{|c|}{ Average |Diff| (Mod) } & 0.02488 \\
\hline & \multicolumn{2}{|c|}{ Std Dev |Diff| (Full) } & 0.0828 & \multicolumn{2}{|c|}{ Std Dev |Diff $\mid$ (Full) } & 0.08901 \\
\hline & \multicolumn{2}{|c|}{ Std Dev |Diff $\mid$ (Mod) } & 0.02183 & \multicolumn{2}{|c|}{ Std Dev |Diff| (Mod) } & 0.02124 \\
\hline
\end{tabular}

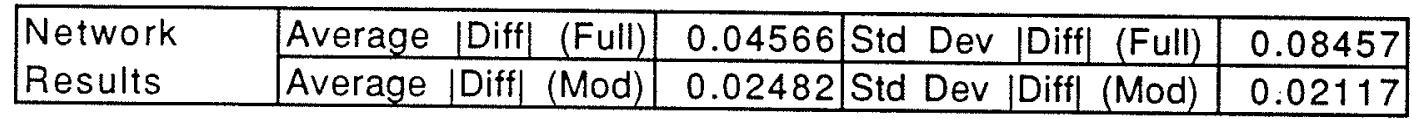


Training Results for V2.2.10.2

(Network 2.10.2, V2 Macro)

\begin{tabular}{|c|c|c|c|c|c|c|}
\hline \multirow[t]{2}{*}{ Destination } & \multicolumn{3}{|c|}{ Neuron o1 (Route 3) } & \multicolumn{3}{|c|}{ Neuron o2 (Route 6) } \\
\hline & Target & Meas & Diff & Target & Meas & Diff \\
\hline$i 1$ & 0.2569 & 0.31316 & -0.0563 & 0.3986 & 0.34247 & 0.05613 \\
\hline i2 & & 0.33448 & -0.3345 & & 0.36095 & -0.361 \\
\hline 13 & 0.1245 & 0.1 & -0.0603 & 0.2663 & 0.2105 & 0.0558 \\
\hline 14 & 0.3486 & 0.3 & 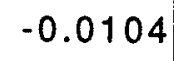 & 0.3 & 0.38 & 0.01085 \\
\hline i5 & 0.4146 & 0.4 & -0.0266 & 0.4924 & 31 & 349 \\
\hline$i 6$ & 0.2335 & 0.1 & 0.05201 & 0.1 & 0.20618 & 89 \\
\hline$i 7$ & 0.249 & 0.25905 & -0.0101 & 0.297 & 0.28 & 976 \\
\hline 18 & 0.3588 & 0.3 & -0.0 & 0.4 & & 181 \\
\hline i9 & 0.4618 & 0.4 & -0. & 0.5 & 0.4 & 376 \\
\hline$\$ 10$ & 0.3524 & 0.3 & 0.05227 & 0.2762 & 0.3 & 22 \\
\hline i11 & 0.3588 & 0.35 & 0.00303 & 0.3793 & 0.38 & -0.0027 \\
\hline & 0.4776 & 0.47662 & 0.00 & 0.4981 & $0.4 \varsigma$ & -0.001 \\
\hline$i$ & 0.6385 & & -0.0056 & 0.659 & 0.65277 & 0.00623 \\
\hline$i 14$ & 0.4405 & 0.3 & 0.0484 & & 0.4153 & -0.051 \\
\hline$i 15$ & 0.4942 & 0.4 & 0.0 & 0.4 & 0.4926 & -0.0193 \\
\hline$i 16$ & 0.6745 & 0.66325 & 0.01125 & 0.6536 & 0.66586 & -0.0123 \\
\hline \multirow{4}{*}{$\begin{array}{l}\text { Neuron } \\
\text { Results }\end{array}$} & \multicolumn{2}{|c|}{ Average $\mid$ Diff $\mid$ (Full) } & 0.04459 & \multicolumn{2}{|c|}{ Average |Diff| (Full) } & 0.04632 \\
\hline & \multirow{2}{*}{\multicolumn{2}{|c|}{\begin{tabular}{|l|l|l|} 
Average & Diff & (Mod) \\
Std Dev & IDiff & (Full) \\
\end{tabular}}} & 0.02526 & \multicolumn{2}{|c|}{ Average |Diff| (Mod) } & 0.02534 \\
\hline & & & 0.08014 & \multirow{2}{*}{\multicolumn{2}{|c|}{\begin{tabular}{|l|l|} 
Std Dev & Diff $\mid$ (Full) \\
Std & Dev \\
Diff $\mid$ (Mod)
\end{tabular}}} & 0.08636 \\
\hline & \multicolumn{2}{|c|}{\begin{tabular}{|l|l|} 
Std Dev & Diff $\mid$ (Fulli) \\
Std Dev $[$ Diff $\mid$ (Mod)
\end{tabular}} & 0.02188 & & & 0.02116 \\
\hline
\end{tabular}

\begin{tabular}{|l|l|r|l|l|}
\hline $\begin{array}{l}\text { Network } \\
\text { Results }\end{array}$ & Average |Diff| (Full) & 0.04545 & Std Dev |Diff $\mid$ (Full) & 0.08196 \\
\cline { 2 - 5 } & Average |Diff $\mid$ (Mod) & 0.0253 & Std Dev |Diff $\mid$ (Mod) & 0.02115 \\
\hline
\end{tabular}


Training Results for V2.2.06.4

(Network 2.06.4, V2 Macro)

\begin{tabular}{|c|c|c|c|c|c|c|}
\hline \multirow[t]{2}{*}{ Destination } & \multicolumn{3}{|c|}{ Neuron o1 (Route 5) } & \multicolumn{3}{|c|}{ Neuron o2 (Route 8) } \\
\hline & Target & Meas & Diff & Target & Meas & Diff \\
\hline$i 1$ & 0.3708 & 0.38399 & -0.0132 & 0.3838 & 0.35059 & 0.03321 \\
\hline i2 & 0.6027 & 0.60233 & 0.00037 & 0.4619 & 0.46229 & -0.0004 \\
\hline i3 & 0.5031 & 0.50783 & -0.0047 & 0.3623 & 0.36139 & 0.00091 \\
\hline i 4 & 0.3088 & 0.30664 & 0.00216 & 0.2418 & 0.25847 & -0.0167 \\
\hline$i 5$ & 0.213 & 0.25767 & -0.0447 & 0.3376 & 0.2794 & 0.0582 \\
\hline i 6 & 0.5481 & 0.54793 & 0.00017 & 0.3776 & 0.38121 & -0.0036 \\
\hline$i 7$ & 0.4084 & 0.39223 & 0.01617 & 0.2379 & 0.27368 & -0.0358 \\
\hline i 8 & 0.4225 & 0.36142 & 0.06108 & 0.1281 & 0.24601 & -0.1179 \\
\hline 19 & & 0.38876 & -0.3888 & & 0.21248 & -0.2125 \\
\hline$i 10$ & 0.6212 & 0.61758 & 0.00362 & 0.3761 & 0.37943 & -0.0033 \\
\hline$i 11$ & 0.5181 & 0.50669 & 0.01141 & 0.273 & 0.29045 & -0.0175 \\
\hline$i 12$ & 0.5989 & 0.58212 & 0.01678 & 0.3045 & 0.30689 & -0.0024 \\
\hline $\mathrm{i} 13$ & 0.7597 & 0.78868 & -0.029 & 0.4653 & 0.45896 & 0.00634 \\
\hline$i 14$ & 0.663 & 0.6663 & -0.0033 & 0.4179 & 0.40769 & 0.01021 \\
\hline$i 15$ & 0.6536 & 0.66154 & -0.0079 & 0.4085 & 0.37938 & 0.02912 \\
\hline$i 16$ & 0.8046 & 0.82517 & -0.0206 & 0.5102 & 0.50217 & 0.00803 \\
\hline \multirow{4}{*}{$\begin{array}{l}\text { Neuron } \\
\text { Results }\end{array}$} & \multicolumn{2}{|c|}{ Average |Diff| (Full) } & 0.03899 & \multicolumn{2}{|c|}{ Average |Diff| (Full) } & 0.03475 \\
\hline & \multicolumn{2}{|c|}{ Average |Diff] (Mod) } & 0.01568 & \multicolumn{2}{|c|}{ Average |Diff| (Mod) } & 0.0229 \\
\hline & \multicolumn{2}{|c|}{\begin{tabular}{|l|l|} 
Std Dev $\mid$ Diff $\mid$ (Full) \\
\end{tabular}} & 0.09478 & \multicolumn{2}{|c|}{ Std Dev |Diff| (Full) } & 0.05608 \\
\hline & \multicolumn{2}{|c|}{ Std Dev |Diff| (Mod) } & 0.01745 & \multicolumn{2}{|c|}{ Std Dev [Diff] (Mod) } & 0.03102 \\
\hline
\end{tabular}

\begin{tabular}{|c|c|c|c|c|c|c|}
\hline \multirow[t]{2}{*}{ Destination } & \multicolumn{3}{|c|}{ Neuron o3 (Route 12) } & \multicolumn{3}{|c|}{ Neuron o4 (Route 13) } \\
\hline & Target & Meas & Diff & Target & Meas & Diff \\
\hline$i 1$ & 0.5985 & 0.59974 & -0.0012 & 0.8306 & 0.84774 & -0.0171 \\
\hline$i 2$ & 0.6191 & 0.62235 & -0.0032 & 0.8512 & 0.84645 & 0.00475 \\
\hline i3 & 0.5195 & 0.50448 & 0.01502 & 0.7516 & 0.76569 & -0.0141 \\
\hline$i 4$ & 0.4565 & 0.43547 & 0.02103 & 0.6886 & 0.70164 & -0.013 \\
\hline$i 5$ & 0.5523 & 0.5509 & 0.0014 & 0.7844 & 0.81159 & -0.0272 \\
\hline i6 & 0.5072 & 0.49724 & 0.00996 & 0.7393 & 0.74685 & -0.0076 \\
\hline$i 7$ & 0.395 & 0.38184 & 0.01316 & 0.6271 & 0.62745 & -0.0003 \\
\hline i 8 & 0.3428 & 0.3317 & 0.0111 & 0.5749 & 0.55588 & 0.01902 \\
\hline$i 9$ & & 0.22271 & -0.2227 & & 0.37734 & -0.3773 \\
\hline$i 10$ & 0.3884 & 0.39953 & -0.0111 & 0.6205 & 0.61139 & 0.00911 \\
\hline$i 11$ & 0.2853 & 0.3072 & -0.0219 & 0.5174 & 0.49998 & 0.01742 \\
\hline $\mathrm{i} 12$ & 0.1664 & 0.24706 & -0.0807 & 0.3985 & 0.35617 & 0.04233 \\
\hline$i 13$ & 0.3272 & 0.26207 & 0.06513 & 0.2377 & 0.27664 & -0.0389 \\
\hline$i 14$ & 0.3851 & 0.40448 & -0.0194 & 0.6172 & 0.60606 & 0.01114 \\
\hline$¡ 15$ & 0.2762 & 0.33001 & -0.0538 & 0.5083 & 0.48314 & 0.02516 \\
\hline 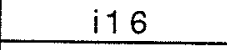 & 0.3712 & 0.33564 & 0.03556 & 0.3929 & 0.41925 & -0.0264 \\
\hline \multirow{4}{*}{$\begin{array}{l}\text { Neuron } \\
\text { Results }\end{array}$} & \multicolumn{2}{|c|}{ Average |Diff| (Full)| } & 0.03665 & \multicolumn{2}{|c|}{ Average |Diff| (Full) } & 0.04068 \\
\hline & \multicolumn{2}{|c|}{ Average |Diff (Mod) } & 0.02425 & \multicolumn{2}{|c|}{ Average |Diff $\mid$ (Mod) } & 0.01824 \\
\hline & \multicolumn{2}{|c|}{\begin{tabular}{|l|l|} 
Std Dev $\mid$ Diff $\mid$ (Full) \\
Std
\end{tabular}} & 0.05481 & \multicolumn{2}{|c|}{ Std Dev |Diff $\mid$ (Full) } & 0.09051 \\
\hline & Std Dev $\mid \mathrm{D}$ & iff (Mod) & 0.02412 & Std Dev $1 D$ & iff) (Mod) & 0.01195 \\
\hline
\end{tabular}

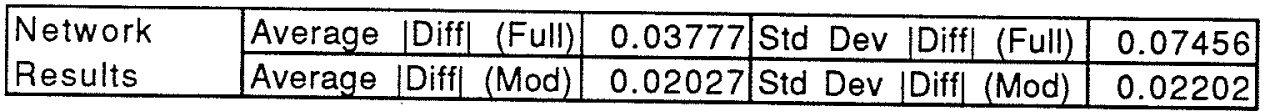


Training Results for V2.2.08.4

(Network 2.08.4, V2 Macro)

\begin{tabular}{|c|c|c|c|c|c|c|}
\hline \multirow[t]{2}{*}{ Destination } & \multicolumn{3}{|c|}{ Neuron o1 (Route 5) } & \multicolumn{3}{|c|}{ Neuron 02 (Route 8) } \\
\hline & Target & Meas & Diff & Target & Meas & Diff \\
\hline i 1 & 0.3708 & 0.38102 & -0.0102 & 0.3838 & 0.35577 & 0.0280 \\
\hline$i 2$ & 0.6027 & 0.60416 & -0.0015 & 0.4619 & 0.46389 & -0.00 \\
\hline i3 & 0.5031 & 0.50401 & -0.0009 & 0.3623 & 0.36782 & -0.0055 \\
\hline i4 & 0.3088 & 0.30029 & 0.00851 & 0.2 & 0.25907 & -0.0173 \\
\hline i 5 & 0.213 & 0.26661 & -0.0536 & 0.3 & 0.28925 & 0.04835 \\
\hline$i 6$ & 0.5 & 0.5502 & -0.0021 & 0.3776 & 0.38232 & -0.0047 \\
\hline i 7 & 0.4084 & 0.39251 & 0.01589 & 0.2379 & 0.27058 & -0.0327 \\
\hline i 8 & 0.4225 & 0.35983 & 0.06267 & 0.1281 & 0.24146 & -0.1134 \\
\hline$i 9$ & & 0.38955 & -0.3896 & & 0.23948 & -0.2395 \\
\hline 110 & 0.6212 & 0.62405 & -0.0029 & 0.3761 & 0.37564 & 0.00046 \\
\hline$\lceil 11$ & 0.5181 & 0.5156 & 0.0025 & 0.273 & 0.28839 & -0.0154 \\
\hline$i 12$ & 0.5989 & 0.59143 & 0.00747 & 0.3045 & 0.3 & 0.004 \\
\hline$\lceil 13$ & 0.7597 & 0.7 & -0.0172 & 0.4653 & 0.4704 & -0.0051 \\
\hline $\mathrm{i}$ & 63 & 0.6 & -0.0133 & 0.4179 & 0.40034 & 0.01756 \\
\hline$i 15$ & 0.6536 & 0.66248 & -0.0089 & 0.4085 & 0.34862 & 0.05988 \\
\hline$i 16$ & 0.8046 & 0.81876 & -0.0142 & 0.5102 & 0.51038 & -0.0002 \\
\hline \multirow{4}{*}{$\begin{array}{l}\text { Neuron } \\
\text { Results }\end{array}$} & \multicolumn{2}{|c|}{ Average |Diff| (Full) } & 0.03821 & \multicolumn{2}{|c|}{ Average |Diff (Full) } & 0.03712 \\
\hline & \multicolumn{2}{|c|}{ Average |Diff $\mid$ (Mod) } & 0.01479 & \multicolumn{2}{|c|}{ Average |Diff| (Mod) } & 0.02363 \\
\hline & \multicolumn{2}{|c|}{ Std Dev |Diff| (Full) } & 0.09538 & \multicolumn{2}{|c|}{\begin{tabular}{|l|l|} 
Std Dev [Diff (Full) \\
\end{tabular}} & 0.06157 \\
\hline & \multicolumn{2}{|c|}{ Std Dev |Diff $\mid$ (Mod) } & 0.01848 & Std Dev 10 & Diff) (Mod) & 0.0307 \\
\hline
\end{tabular}

\begin{tabular}{|c|c|c|c|c|c|c|}
\hline \multirow[t]{2}{*}{ Destination } & \multicolumn{3}{|c|}{ Neuron 03 (Route 12) } & \multicolumn{3}{|c|}{ Neuron o4 (Route 13) } \\
\hline & Target & Meas & Diff & Target & Meas & Diff \\
\hline i1 & 0.5985 & 0.60624 & -0.0077 & 0.8306 & 0.84239 & -0.0118 \\
\hline$i 2$ & 0.6191 & 0.62525 & -0.0061 & 0.8512 & 0.84649 & 0.0047 \\
\hline i3 & 0.5195 & 0.51206 & 0.00744 & 0.7516 & 0.76105 & -0.0094 \\
\hline 14 & 0.4 & 0.4 & 0.01644 & 0.6886 & 0.7017 & -0.0131 \\
\hline i 5 & 0.5 & 0. & -0.004 & 44 & 0.8 & 198 \\
\hline i 6 & 0.5 & 0.4 & 0.00726 & 0.7 & 0.7 & -0.0091 \\
\hline i 7 & 0.395 & 0.3 & 0.01481 & 71 & 0.6 & \\
\hline i 8 & 0.3428 & 0.3 & 0.01294 & 0.5749 & 0.56 & \\
\hline i9 & & 0.2 & -0.2966 & & 0.51 & \\
\hline$\lceil 10$ & 0.3884 & 0.3 & -0.0068 & & 0.6 & \\
\hline$i 1$ & 0.2853 & 0.2 & -0.0141 & & 0.5 & 0. \\
\hline $\mathrm{i}$ & 0.16 & 0.2 & -0.073 & 0.2 & 0.3 & 0. \\
\hline & 2 & 0.2 & 0.04732 & & 0.2 & \\
\hline & 0.38 & 0.3 & -0.0109 & 0.6 & 0.6 & \\
\hline i1 & 0.2762 & 0.3 & -0.0542 & 0.5 & 0.2 & 0. \\
\hline & 0.3712 & 0.34606 & 0.02514 & 0.3929 & 0.40855 & -0 . \\
\hline \multirow{4}{*}{$\begin{array}{l}\text { Neuron } \\
\text { Results }\end{array}$} & \multicolumn{2}{|c|}{ Average |Diff| (Full) } & 0.03779 & \multicolumn{2}{|c|}{ Average |Diff| (Full) } & 0.04381 \\
\hline & \multicolumn{2}{|c|}{ Average [Diff] (Mod) } & 0.02054 & \multicolumn{2}{|c|}{ Average $\mid$ Diff $\mid$ (Mod) } & 0.01263 \\
\hline & \multicolumn{2}{|c|}{ Std Dev |Diff| (Full) } & 0.07186 & \multicolumn{2}{|c|}{ Std Dev |Diff] (Full) } & 0.12492 \\
\hline & \multicolumn{2}{|c|}{\begin{tabular}{|l|} 
Std Dev IDiff] (Mod) \\
\end{tabular}} & 0.02079 & \multicolumn{2}{|c|}{ Std Dev |Diff| (Mod) } & 0.00788 \\
\hline \multirow{2}{*}{$\begin{array}{l}\text { Network } \\
\text { Results }\end{array}$} & \multicolumn{2}{|c|}{\begin{tabular}{|l|l|} 
Average |Diff $\mid$ (Full) \\
\end{tabular}} & 0.03923 & \multirow{2}{*}{\multicolumn{2}{|c|}{\begin{tabular}{|l|l|l|} 
Std Dev $\mid$ Diff $\mid$ (Full) \\
Std Dev $\mid$ Diff $\mid$ (Mod)
\end{tabular}}} & \\
\hline & Average 10 & iff (Mod) & 0.0179 & & & 0.02101 \\
\hline
\end{tabular}


Training Results for V2.2.10.4

(Network 2.08.4, V2 Macro)

\begin{tabular}{|c|c|c|c|c|c|c|}
\hline \multirow[t]{2}{*}{ Destination } & \multicolumn{3}{|c|}{ Neuron o1 (Route 5) } & \multicolumn{3}{|c|}{ Neuron o2 (Route 8) } \\
\hline & Target & Meas & Diff & Target & Meas & Diff \\
\hline$i 1$ & 0.3708 & 0.3779 & -0.0071 & 0.3838 & 0.35714 & 0.02666 \\
\hline$i 2$ & 0.6027 & 0.60212 & 0.00058 & 0.4619 & 0.46571 & -0.0038 \\
\hline i3 & 0.5031 & 0.50546 & -0.0024 & 0.3623 & 0.36542 & -0.0031 \\
\hline i4 & 0.3088 & 0.31018 & -0.0014 & 0.2418 & 0.26116 & -0.0194 \\
\hline i5 & 0.213 & 0.24618 & -0.0332 & 0.3376 & 0.29302 & 0.04458 \\
\hline$i 6$ & 0.5481 & 0.55125 & -0.0031 & 0.3776 & 0.37959 & -0.002 \\
\hline i7 & 0.4084 & 0.40363 & 0.00477 & 0.2379 & 0.26359 & -0.0257 \\
\hline i 8 & 0.4225 & 0.37297 & 0.04953 & 0.1281 & 0.23745 & -0.1094 \\
\hline i9 & & 0.45799 & -0.458 & & 0.31976 & -0.3198 \\
\hline$i 10$ & 0.6212 & 0.62135 & -0.0001 & 0.3761 & 0.37616 & $-6 \mathrm{E}-05$ \\
\hline $\mathrm{i} 11$ & 0.5181 & 0.50856 & 0.00954 & 0.273 & 0.28921 & -0.0162 \\
\hline $\mathrm{i} 12$ & 0.5989 & 0.59116 & 0.00774 & 0.3045 & 0.29978 & 0.00472 \\
\hline$i 13$ & 0.7597 & 0.77617 & -0.0165 & 0.4653 & 0.4692 & -0.0039 \\
\hline ¡14 & 0.663 & 0.67276 & -0.0098 & 0.4179 & 0.40156 & 0.01634 \\
\hline 115 & 0.6536 & 0.66086 & -0.0073 & 0.4085 & 0.38204 & 0.02646 \\
\hline$\$ 16$ & 0.8046 & 0.82075 & -0.0162 & 0.5102 & 0.50839 & 0.00181 \\
\hline \multirow{4}{*}{$\begin{array}{l}\text { Neuron } \\
\text { Results }\end{array}$} & \multicolumn{2}{|c|}{ Average |Diff $\mid$ (Full) } & 0.03919 & \multicolumn{2}{|c|}{\begin{tabular}{|l|l|l|} 
Average & Diff & (Full) \\
\end{tabular}} & 0.03899 \\
\hline & \multicolumn{2}{|c|}{ Average |Diff| (Mod) } & 0.01127 & \multicolumn{2}{|c|}{ Average [Diff] (Mod) } & 0.02027 \\
\hline & \multicolumn{2}{|c|}{ Std Dev |Diff $\mid$ (Full) } & 0.11244 & \multicolumn{2}{|c|}{ Std Dev Diff $\mid$ (Full) } & 0.07955 \\
\hline & \multicolumn{2}{|c|}{ Std Dev |Diff $\mid$ (Mod) } & 0.01355 & \multicolumn{2}{|c|}{ Std Dev |Diff] (Mod) } & 0.02781 \\
\hline
\end{tabular}

\begin{tabular}{|c|c|c|c|c|c|c|}
\hline \multirow[t]{2}{*}{ Destination } & \multicolumn{3}{|c|}{ Neuron o3 (Route 12) } & \multicolumn{3}{|c|}{ Neuron o4 (Route 13) } \\
\hline & Target & Meas & Diff & Target & Meas & Diff \\
\hline$i 1$ & 0.5985 & 0.60495 & -0.0064 & 0.8306 & 0.8447 & -0.0141 \\
\hline$i 2$ & 0.6191 & 0.62475 & -0.0057 & 0.8512 & 0.84417 & 0.00703 \\
\hline i3 & 0.5195 & 0.51082 & 0.00868 & 0.7516 & 0.76138 & -0.0098 \\
\hline ¡ 4 & 0.4565 & 0.43557 & 0.02093 & 0.6886 & 0.70075 & -0.0122 \\
\hline i5 & 0.5523 & 0.56083 & -0.0085 & 0.7844 & 0.80292 & -0.0185 \\
\hline i 6 & 0.5072 & 0.49889 & 0.00831 & 0.7393 & 0.74 & -0.0096 \\
\hline$i 7$ & 0.395 & 0.37291 & 0.02209 & 0.6271 & 0.63743 & -0.0103 \\
\hline i 8 & 0.3428 & 0.32404 & 0.01876 & 0.5749 & 0.56832 & 0.00658 \\
\hline i9 & & 0.44419 & -0.4442 & & 0.70048 & -0.7005 \\
\hline$\lceil 10$ & 0.3884 & 0.39882 & -0.0104 & 0.6205 & 0.6 & 0.00571 \\
\hline$i 11$ & 0.2853 & 0.30611 & -0.0208 & 0.5174 & 0.50194 & 0.01546 \\
\hline$\lceil 12$ & 0.1664 & 0.24297 & -0.0766 & 0.3985 & 0.36923 & 0.02927 \\
\hline$\lceil 13$ & 0.3272 & 0.2716 & 0.0556 & 0.2377 & 0.2 & -0.031 \\
\hline ¡14 & 0.3851 & 0.40075 & -0.0157 & 0.6172 & 0.61169 & 0.00551 \\
\hline$\lceil 15$ & 0.2762 & 0.33292 & -0.0567 & 0.5083 & 0.48511 & 0.02319 \\
\hline$i 8$ & 0.3712 & 0.34059 & 0.03061 & 0.3929 & 0.41279 & -0.0199 \\
\hline \multirow{4}{*}{$\begin{array}{l}\text { Neuron } \\
\text { Results }\end{array}$} & \multicolumn{2}{|c|}{ Average |Diff| (Full) } & 0.05062 & \multicolumn{2}{|c|}{ Average |Diff (Full) } & 0.05741 \\
\hline & \multicolumn{2}{|c|}{\begin{tabular}{|l|l|} 
Average $\mid$ Diff $\mid$ (Mod) \\
\end{tabular}} & 0.02439 & \multicolumn{2}{|c|}{ Average |Diff| (Mod) } & 0.01454 \\
\hline & \multicolumn{2}{|c|}{\begin{tabular}{|l|l|l|} 
Std Dev $\mid$ Diff $\mid$ (Full) \\
\end{tabular}} & 0.10701 & \multicolumn{2}{|c|}{ Std Dev |Diff| (Full) } & 0.17167 \\
\hline & \multicolumn{2}{|c|}{ Std Dev |Diff $\mid$ (Mod) } & 0.02162 & \multicolumn{2}{|c|}{ Std Dev [Diff] (Mod) } & 0.00827 \\
\hline
\end{tabular}

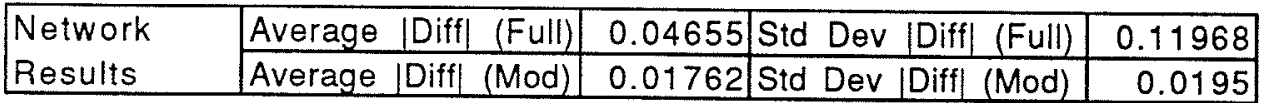


Training Results for V2.2.06.6

(Network 2.06.6, V2 Macro)

\begin{tabular}{|c|c|c|c|c|c|c|c|c|c|}
\hline \multirow[t]{2}{*}{ Destination } & \multicolumn{3}{|c|}{ Neuron o1 (Route 7) } & \multicolumn{3}{|c|}{ Neuron o2 (Route 8) } & \multicolumn{3}{|c|}{ Neuron o3 (Route 10) } \\
\hline & Target & Meas & Diff & Target & Mivas & Diff & Target & Meas & Diff \\
\hline$i 1$ & 0.3763 & 0.32439 & 0.05191 & 0.4256 & 0.45136 & -0.0258 & 0.4882 & 0.446 & $\overline{42}$ \\
\hline$i 2$ & 0.3588 & 0.35306 & 0.00574 & 0.5 & 0.50846 & -0.0048 & 0.3793 & 0.35668 & 0.02262 \\
\hline i3 & 0.2592 & 04 & -0.0018 & 0.4041 & 0.3439 & 0.0602 & 559 & 0.40491 & -0.049 \\
\hline$i 4$ & & 0.1 & 0.03587 & 0.2 & 0.25905 & 0.02455 & 0.2 & & \\
\hline i5 & & 0.32 & 0.00587 & 0.3 & 0.3434 & 0. & 82 & 37 & 194 \\
\hline i6 & 0.2 & 0.29888 & -0.0244 & 0.4 & 0.44557 & -0.0262 & 47 & & 876 \\
\hline i7 & 0.1 & 0.16 & -0.0 & 0.2797 & 506 & 0.06464 & 0.2 & & 076 \\
\hline 18 & 0.2 & 0.24108 & 0.00342 & 0.1699 & 0.2 & -0 & & & -0 \\
\hline i9 & 0.3 & 0.30476 & 0.04284 & 0.273 & & & & 0.5 & 0. \\
\hline$\$ 10$ & 0.3933 & 0.3628 & 0.0305 & 0.5382 & 0.5 & 0.0 & 0.1281 & 0. & -0 \\
\hline $\mathrm{i}$ & & 0.02144 & -0.0214 & & 0.0 & -0.0239 & & 0.01639 & -0 \\
\hline & & 0.4 & -0. & 0.3 & 0.3 & -0.0096 & & & \\
\hline & & 0.5 & -0.0005 & 0.4857 & 0.4 & 0.0 & 21 & & \\
\hline$i 14$ & 0.4815 & 0.50309 & -0.0216 & 0.6331 & 0.5 & 0.0 & 0.2526 & 68 & 101 \\
\hline$i 15$ & 0.5307 & 0.52237 & 0.00833 & 0.456 & 0.2 & -0 & 0.3615 & 0.36893 & -0.0074 \\
\hline & 0.6266 & 0.63594 & -0.0093 & 0.552 & 0.60312 & -0.0 & 0.5418 & 0.50516 & 0.03664 \\
\hline \multirow{4}{*}{$\begin{array}{l}\text { Neuron } \\
\text { Results }\end{array}$} & \multicolumn{2}{|c|}{ Average (Diff) (Full) } & 0.0185 & \multicolumn{2}{|c|}{\begin{tabular}{|l|l|l|} 
Average & Diff & (Full) \\
\end{tabular}} & 0.03239 & \multicolumn{2}{|c|}{ Average [Diff] (Full) } & 0.02881 \\
\hline & \multicolumn{2}{|c|}{ Average |Diff| (Mod) } & 0.0183 & \multicolumn{2}{|c|}{ Average |Diff| (Mod) } & 0.03296 & \multicolumn{2}{|c|}{ Average |Diff| (Mod) } & \\
\hline & \multicolumn{2}{|c|}{ Std Dev [Diff] (Full) } & 0.01634 & \multicolumn{2}{|c|}{ Std Dev [Diff] (Full) } & 0.02269 & \multicolumn{2}{|c|}{ Std Dev [Diff| (Full) } & \\
\hline & \multicolumn{2}{|c|}{ Std Dev $\mid$ Diff $\mid$ (Mod) } & 0.01689 & \multicolumn{2}{|c|}{ Std Dev |Diff| (Mod) } & 0.02337 & \multicolumn{2}{|c|}{ Std Dev [Diff] (Mod) } & 0.02023 \\
\hline
\end{tabular}

\begin{tabular}{|c|c|c|c|c|c|c|c|c|c|}
\hline \multirow[t]{2}{*}{ Destination } & \multicolumn{3}{|c|}{ Neuron o4 (Route 12) } & \multicolumn{3}{|c|}{ Neuron o5 (Route 14) } & \multicolumn{3}{|c|}{ Neuron o6 (Route 15) } \\
\hline & Tars & Meas & Diff & Target & Meas & Diff & Target & Meas & Diff \\
\hline$i 1$ & 0.5759 & 0.6301 & -0.0542 & 0.6182 & 0.60604 & 0.01216 & 0.7023 & 0.67104 & 0.0312 \\
\hline i2 & 0.6541 & 0.63262 & 0.02148 & 0.5092 & 0.50591 & 0.00329 & 0.6088 & 0.61179 & \\
\hline i3 & 0.5545 & 0.48891 & 0.06559 & 59 & 2778 & -0.0419 & 0.5854 & & 0.0297 \\
\hline ¡4 & 0.4339 & 0.46464 & .0 .0307 & 0. & 517 & 0.03103 & 03 & 0 . & -0.03 \\
\hline$i 5$ & 0.4732 & 0.464 & 0.0092 & 0.712 & 44 & 0.0376 & 97 & & \\
\hline 16 & 0.5696 & 0. & 0.0406 & & 0.3 & 0.02437 & 65 & 97 & \\
\hline i 7 & 0.43 & 0.40 & 0.02381 & 0.5 & 0.5 & -0.0187 & 564 & 16 & \\
\hline i 8 & 0.3203 & 0.27664 & 0.0 & 0.5 & 0.5 & 0.02116 & 67 & 0 . & \\
\hline i9 & 0.2853 & 0.30 & -0.0213 & 0.53 & 96 & -0.016 & 0. & & 274 \\
\hline i10 & 0.4871 & 0.50336 & -0.0163 & 0.2944 & 0.2 & 0.03073 & 0.3939 & 0.3 & 0.0 \\
\hline$i 11$ & & 0.01979 & -0.0198 & & 0.0 & -0 & & & -0.017 \\
\hline$i 12$ & 39 & 0.2047 & -0.0608 & 0.3 & 0.3 & 0.02697 & & 0.2 & \\
\hline i13 & 47 & & 22 & 0.5 & 0.5 & -0.0084 & 0.4 & & \\
\hline$i$ & 0.3 & 0.34563 & 0.01697 & 0.1699 & 0.2 & -0.0716 & 95 & 0.2 & 0. \\
\hline$\lceil 15$ & 0.2536 & 0.19728 & 0.05632 & 0.2789 & 0.2 & 0.01945 & 0.1605 & 37 & -0.0232 \\
\hline$i 16$ & 0.3496 & 0.34465 & 0.00495 & 0.4591 & 0.41313 & 597 & 0.3408 & 0.32935 & 0.01145 \\
\hline \multirow{4}{*}{$\begin{array}{l}\text { Neuron } \\
\text { Results }\end{array}$} & \multicolumn{2}{|c|}{ Average |Diff| (Full) } & 0.03174 & \multicolumn{2}{|c|}{ Average |Diff| (Full) } & 0.02659 & \multicolumn{2}{|c|}{ Average |Diff| (Full) } & 0.02025 \\
\hline & \multicolumn{2}{|c|}{ Average $\mid$ Diff $\mid$ (Mod) } & 0.03254 & \multicolumn{2}{|c|}{ Average |Diff| (Mod) } & 0.02729 & \multicolumn{2}{|c|}{ Average |Diff| (Mod) } & \\
\hline & \multicolumn{2}{|c|}{ Std Dev |Diff $\mid$ (Full) } & 0.01918 & \multicolumn{2}{|c|}{ Std Dev |Diff| (Full) } & 683 & \multicolumn{2}{|c|}{ Std Dev /Diff (Full) } & \\
\hline & \multicolumn{2}{|c|}{ Std Dev [Diff (Mod) } & 0.01957 & \multicolumn{2}{|c|}{ Std Dev |Diff (Mod) } & 0.01718 & \multicolumn{2}{|c|}{ Std Dev |Diff| (Mod) } & 0.01261 \\
\hline
\end{tabular}

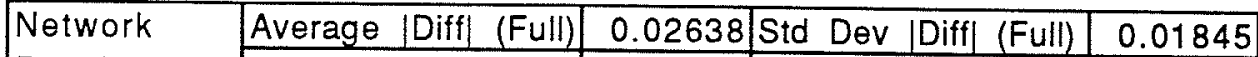

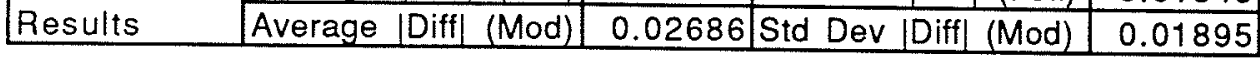


Training Results for V2.2.08.6

(Network 2.08.6, V2 Macro)

\begin{tabular}{|c|c|c|c|c|c|c|c|c|c|}
\hline \multirow[t]{2}{*}{ Destination } & \multicolumn{3}{|c|}{ Neuron 01 (Route 7) } & \multicolumn{3}{|c|}{ Neuron 02 (Route 8 ) } & \multicolumn{3}{|c|}{ Neuron 03 (Route 10) } \\
\hline & Target & Meas & Diff & Target & Meas & Diff & Target & Meas & Diff \\
\hline$i 1$ & 0.3763 & 0.34989 & 0.02641 & 0.4256 & 0.44766 & -0.0221 & 0.4882 & 0.50168 & 0.013 \\
\hline i 2 & 0.3588 & 0.35131 & 0.00749 & 0.5037 & 0.51887 & -0.0152 & 0.3793 & 0.34988 & 0.0294 \\
\hline i3 & 0.2592 & 0.278 & -0.0188 & 0.4041 & 0.40148 & 0.00262 & 0.3559 & 0.34364 & 0.0122 \\
\hline$i 4$ & 0.2343 & 0.23981 & -0.0055 & 0.2 & 0.27888 & 0.00472 & 62 & 0.4 & -0.0056 \\
\hline i 5 & 0.3301 & 644 & -0.0163 & 0.3 & 0.36482 & 0.01458 & 0.582 & 0.6 & -0.0 \\
\hline$i 6$ & 0.2745 & 0.27247 & 0.00203 & 0.4 & 0.42 & -0.0025 & 0.247 & & -0.000 \\
\hline$i 7$ & 0. & 916 & -0.0444 & 0.2797 & 0.2 & 0.0 & & 33 & 63 \\
\hline i 8 & 0.2445 & 0.21329 & 0.03121 & 0.1 & & -0. & 0.4964 & 61 & -0 \\
\hline i 9 & 0.3476 & 0.35041 & -0.0028 & 0.273 & 0.28958 & -0.0166 & 0.5995 & 66 & 0.0 \\
\hline ¡10 & 0.3933 & 0.37683 & 0.01647 & 0.5382 & 0.53924 & -0.001 & 0.1281 & 0.2 & -0 \\
\hline$i 11$ & & 0.12014 & -0.1201 & & 0.1 & -0.1441 & & 0.1 & -0 \\
\hline$i 12$ & 0.4209 & 0.42925 & -0.0084 & 0.3 & 0.3 & 0.0 & 0.4713 & 0.4 & -0 \\
\hline 113 & 0.5603 & 0.56671 & -0. & 0.4 & 84 & 0.0073 & 0.6321 & & 0. \\
\hline & 0. & 0.5 & -0.0 & 0.6331 & 0.62399 & 0.00911 & 0.2 & & \\
\hline & 0.5307 & 0.5 & 0.00381 & 0.456 & 0.46873 & -0 & & & -0.007 \\
\hline$\$ 16$ & 0.6266 & 0.62308 & 0.00352 & 0.552 & 0.56157 & -0. & 0.5418 & 0.54554 & -0.0037 \\
\hline \multirow{4}{*}{$\begin{array}{l}\text { Neuron } \\
\text { Results }\end{array}$} & \multicolumn{2}{|c|}{ Average |Diff| (Full) } & 0.02102 & \multicolumn{2}{|c|}{\begin{tabular}{|l|l|l|} 
Average & Diff & (Full) \\
\end{tabular}} & 218 & \multicolumn{2}{|c|}{ Average |Diff| (Full) } & 0.02307 \\
\hline & \multicolumn{2}{|c|}{ Average $\mid$ Diff $\mid$ (Mod) } & 0.01441 & \multicolumn{2}{|c|}{ Average |Diff (Mod) } & 0.01364 & \multicolumn{2}{|c|}{ Average |Diff| (Mod) } & 0.01792 \\
\hline & \multicolumn{2}{|c|}{ Std Dev Diff (Full) } & 0.02903 & \multicolumn{2}{|c|}{ Std Dev |Diff| (Full) } & 0.03425 & \multicolumn{2}{|c|}{ Std Dev |Diff| (Full) } & 917 \\
\hline & \multicolumn{2}{|c|}{ Std Dev |Diff| (Mod) } & 0.01243 & \multicolumn{2}{|c|}{ Std Dev [Diff] (Mod) } & 0.01083 & \multicolumn{2}{|c|}{ Std Dev |Diff| (Mod) } & 0.02138 \\
\hline
\end{tabular}

\begin{tabular}{|c|c|c|c|c|c|c|c|c|c|}
\hline \multirow[t]{2}{*}{ Destination } & \multicolumn{3}{|c|}{ Neuron o4 (Route 12) } & \multicolumn{3}{|c|}{ Neuron o5 (Route 14) } & \multicolumn{3}{|c|}{ Neuron o6 (Route 15) } \\
\hline & Taro & Meas & Diff & & Meas & $4 \pi 1$ & tar & Meas & DIIT \\
\hline$i 1$ & 0.5759 & 0.59753 & -0.0216 & 82 & 0.63449 & -0.0163 & 0.7023 & 0.66152 & 0.0407 \\
\hline i2 & 0.6541 & 0.6518 & 0.0023 & 92 & 02 & -0.0028 & 0.6088 & 0.62874 & -0.019 \\
\hline i3 & 0.5545 & 0.5615 & -0.007 & & 29 & -0.0114 & 0.5854 & 0.57 & 0.0058 \\
\hline i 4 & 0.4339 & 794 & -0.004 & 0.6162 & 0.60 & 0.0 & 03 & 0.5 & 12 \\
\hline$i 5$ & 0.4 & 0.2 & -0.0059 & 0.712 & 0.67723 & 0.0 & & & \\
\hline i6 & 0.5696 & 0.5 & 0.02572 & 0.3 & 0.3 & -0. & 0.4765 & & \\
\hline i7 & 0.43 & 0.44009 & -0.0101 & 0.5166 & 0.5 & -0 & 64 & & 0 . \\
\hline i 8 & 0.3203 & 0.29372 & 0.02658 & 0.565 & 0.5 & 0.0 & 0.2 & 0. & -0 \\
\hline i9 & 0.2853 & 0.27489 & 0.01041 & 0.53 & 0. & -0 & & & -0 \\
\hline$¡ 10$ & 0.4871 & 0.4 & -0.0061 & 0.2944 & 0.2 & 0.0 & 0.3939 & 0.3 & 0.1 \\
\hline$i 1$ & & 0.1 & -0 & & 0.1 & -0 & & 0.13107 & -0 \\
\hline ¡12 & & 0.1 & -0.0 & 0. & 0.3 & 0.00 & & & \\
\hline i1 & 7 & & -0. & 0.5494 & 0.56 & -0.0 & 0.4 & 0.4 & \\
\hline$i 14$ & 0.3626 & 0.37717 & -0.0146 & 0.1699 & 0.1 & -0.02 & 395 & 0.2 & 0.0 \\
\hline i15 & 0.2536 & 0.21362 & 0.03998 & 0.2789 & 0.2 & 0.013 & 05 & 0.1 & -0.038 \\
\hline$\lceil 16$ & 0.3496 & 0.33426 & 0.01534 & 0.4591 & 0.45888 & & 0.3408 & 0.35561 & -0.0148 \\
\hline \multirow{4}{*}{$\begin{array}{l}\text { Neuron } \\
\text { Results }\end{array}$} & \multicolumn{2}{|c|}{$\begin{array}{ll}\text { Average } \mid \text { Diff } & \text { (Full) } \\
\end{array}$} & 0.02493 & \multicolumn{2}{|c|}{ Average |Diff $\mid$ (Full) } & 0.01974 & \multicolumn{2}{|c|}{ Average |Diff | (Full) } & 0.0246 \\
\hline & \multicolumn{2}{|c|}{ Average |Diff $\mid$ (Mod) } & 0.01731 & \multicolumn{2}{|c|}{ Average |Diff| (Mod) } & 0.01328 & \multicolumn{2}{|c|}{ Average |Diff| (Mod) } & \\
\hline & \multicolumn{2}{|c|}{ Std Dev [Diff] (Full) } & 0.03313 & \multicolumn{2}{|c|}{ Std Dev IDiff (Fuli) } & 0.0 & \multicolumn{2}{|c|}{ Std Dev |Diff| (Full) } & \\
\hline & \multicolumn{2}{|c|}{ Std Dev |Diff $\mid$ (Mod) } & 0.01351 & \multicolumn{2}{|c|}{ Std Dev |Diff $\mid$ (Mod) } & 0.01008 & \multicolumn{2}{|c|}{ Std Dev |Diff| (Mod) } & 0.0113 \\
\hline
\end{tabular}

\begin{tabular}{|l|l|l|l|l|l|l|}
\hline Network & Average $\mid$ Diff $\mid$ (Full) & 0.02253 & Std Dev |Diff & (Full) & 0.02994 \\
\cline { 2 - 7 } & Average Liff & (Mod) & 0.01569 & Std Dev DDiff (Mod & 0.01354 \\
\hline
\end{tabular}

\begin{tabular}{|l|l|l|l|l|l|}
\hline Results & Average LDiff (Mod) & 0.01569 & Std Dev DDiff (Mod) & 0.01354 \\
\hline
\end{tabular} 
Training Results for V2.2.10.6

(Network 2.10.6, V2 Macro)

\begin{tabular}{|c|c|c|c|c|c|c|c|c|c|}
\hline \multirow[t]{2}{*}{ Destination } & \multicolumn{3}{|c|}{ Neuron o1 (Route 7) } & \multicolumn{3}{|c|}{ Neuron 02 (Route 8) } & \multicolumn{3}{|c|}{ Neuron o3 (Route 10) } \\
\hline & Targ & Meas & Diff & Target & Meas & Diff & Target & Meas & Diff \\
\hline$i 1$ & 0.3763 & 0.35425 & 0.02205 & 0.4256 & 0.45679 & -0.0312 & 0.4882 & 0.48911 & -0.000 \\
\hline $\mathrm{i} 2$ & 0.3588 & 0.35134 & 0.00746 & 0.5037 & 0.52054 & -0.0168 & 0.3793 & 0.33574 & 0.0435 \\
\hline i3 & 0.2592 & & -0.0217 & 0.4041 & 0.40064 & 0.00 & 59 & 0.34 & 0.01018 \\
\hline i 4 & 0.2 & 0.2 & 0.00131 & 0.2 & 0.27671 & 0.0 & 0.4 & & \\
\hline is & & 0.3 & -0.0257 & 0.5 & 0.36 & 0.00967 & & & \\
\hline i6 & 0.2 & 0.27319 & 0.00131 & 0. & 0.40513 & 0.01427 & 47 & & 21 \\
\hline i 7 & 0.1348 & 0.16 & -0.0 & 0.2797 & 0.22475 & 495 & 0.3 & & -0 \\
\hline i 8 & 0.2 & 0.2 & 0.03583 & 0.1699 & 0.2 & -0 & 4 & & -0 \\
\hline 19 & 0.3 & 0.34698 & 0.00062 & 0.273 & 0.2 & & & & 0.1 \\
\hline i10 & 0.3933 & 0.39468 & -0.0014 & 0.5382 & & 0.0 & 0.1281 & & -0 . \\
\hline$i 11$ & & 0.07305 & -0.0731 & & 0.0 & -0.0821 & & 0.0 & -0.076 \\
\hline$i 12$ & & 0.44293 & -0 & 0.3 & & 0.0225 & & & \\
\hline & & 0.5 & -0.0032 & 0.4857 & 0.4 & 0.0 & & & 0.1 \\
\hline & 0.4815 & 0.49609 & -0.0146 & 0.6331 & 0.63187 & 0.0 & 0.2526 & 23 & 0.0 \\
\hline$i 15$ & 0.5307 & 0.51708 & 0.01362 & 0.456 & 0.46 & -0 & 0.3615 & 47 & -0.00 \\
\hline$i 1$ & 0.6266 & 0.62369 & 0.00291 & 0.552 & 0.56127 & -0 . & 0.5418 & 0.54884 & -0.007 \\
\hline \multirow{4}{*}{$\begin{array}{l}\text { Neuron } \\
\text { Results }\end{array}$} & \multicolumn{2}{|c|}{ Average |Diff| (Full) } & 0.0175 & \multicolumn{2}{|c|}{ Average |Diff | (Full) } & 0.02166 & \multicolumn{2}{|c|}{\begin{tabular}{|l|l|l|} 
Average $\mid$ Diff & (Full) \\
\end{tabular}} & 0.02788 \\
\hline & \multicolumn{2}{|c|}{ Average $\mid$ Diff $\mid$ (Mod) } & 0.01379 & \multicolumn{2}{|c|}{ Average |Diff] (Mod) } & 0.01763 & \multicolumn{2}{|c|}{ Average [Diff] (Mod) } & \\
\hline & \multirow{2}{*}{\multicolumn{2}{|c|}{ Std Dev |Diff| (Full) }} & 0.01898 & \multicolumn{2}{|c|}{ Std Dev |Diff| (Full) } & 0.02223 & \multicolumn{2}{|c|}{\begin{tabular}{|l|l|l|} 
Std Dev $\mid$ Diff $\mid$ (Full) \\
\end{tabular}} & \\
\hline & & Std Dev |Diff| (Mod) & 0.01227 & \multicolumn{2}{|c|}{ Std Dev |Diff $\mid$ (Mod) } & 0.01584 & \multicolumn{2}{|c|}{ Std Dev |Diff| (Mod) } & 0.02907 \\
\hline
\end{tabular}

\begin{tabular}{|c|c|c|c|c|c|c|c|c|c|}
\hline \multirow[t]{2}{*}{ Destination } & \multicolumn{3}{|c|}{ Neuron o4 (Route 12) } & \multicolumn{3}{|c|}{ Neuron o5 (Route 14) } & \multicolumn{3}{|c|}{ Neuron o6 (Route 15) } \\
\hline & Target & Meas & Diff & Target & Meas & Diff & Ta & Meas & Diff \\
\hline$i 1$ & 0.5759 & 0.61042 & -0.0345 & 0.6182 & 0.62439 & -0.0062 & 023 & 08 & .0412 \\
\hline i2 & 0.6541 & 0.66451 & -0.0104 & 0.5092 & 0.49877 & 0.01043 & 0.6088 & 0.63245 & 0.023 \\
\hline i3 & 0.5545 & 0.56398 & -0.0095 & 0.4859 & 0.49 & -0.0081 & 54 & 0.57875 & 0.0066 \\
\hline ¡ 4 & 0.4339 & 0.44254 & -0 & 0.6162 & 0.60665 & 0.00955 & 0.5603 & 0.5735 & -0.013 \\
\hline i5 & 0.4732 & 0.46698 & 0.00622 & 0.712 & 0.67986 & 0.03214 & 97 & 0.6 & -0.005 \\
\hline i 6 & 0.5696 & 0.53378 & 0.0 & 0.3769 & 0.3 & -0.022 & 65 & 0. & \\
\hline i 7 & 0.43 & 0.4 & 0.0 & 0.5 & 0.5 & -0.0236 & & 4766 & \\
\hline i 8 & 0.32 & 0.30773 & 0.0 & 0.565 & 0.5 & 0.0 & 0.4 & 47 & -0 \\
\hline i9 & 0.2853 & 0.29624 & -0. & 0.53 & 0.55496 & -0.025 & 0.4 & 0. & -0 \\
\hline$i 10$ & 0.4871 & 0.47447 & 0.01263 & 0.2944 & 0.28239 & 0.01201 & 0.3939 & 0.5 & 0.0 \\
\hline$i 11$ & & 0.08528 & -0.0853 & & 0.08725 & -0 & & & -0 . \\
\hline 112 & 0.1439 & 0.1722 & -0.0283 & 0.3 & 0.5 & 709 & & & \\
\hline$i 13$ & 0.3047 & 0.32577 & -0. & 0.5 & 0.5 & -0.0185 & 0.4311 & & \\
\hline & 0.3626 & 0.38 & -0 & 0.1699 & 0.1 & -0.0008 & 0.2 & & \\
\hline & 0.2536 & 0.22715 & 0.02645 & 0.2789 & 0.2 & 0.0 & 05 & 0.21797 & -0.0575 \\
\hline & 0.3496 & 0.32831 & 0.02129 & 0.4591 & 0.46231 & -0 . & 0.3408 & 0.3557 & -0.0149 \\
\hline \multirow{4}{*}{$\begin{array}{l}\text { Neuron } \\
\text { Results }\end{array}$} & \multicolumn{2}{|c|}{ Average [Diff] (Full) } & 0.02187 & \multicolumn{2}{|c|}{ Average |Diff (Full) } & 393 & \multicolumn{2}{|c|}{ Average [Diff] (Full) } & 0.0255 \\
\hline & \multicolumn{2}{|c|}{ Average |Diff $\mid$ (Mod) } & 0.01764 & \multicolumn{2}{|c|}{ Average |Diff] (Mod) } & 0.01224 & \multicolumn{2}{|c|}{ Average $\mid$ Diff $\mid$ (Mod) } & 0.02128 \\
\hline & \multicolumn{2}{|c|}{ Std Dev |Diff| (Full) } & 0.01983 & \multicolumn{2}{|c|}{ Std Dev |Diff| (Full) } & 0.02097 & \multicolumn{2}{|c|}{ Std Dev |Diff| (Full) } & \\
\hline & \multicolumn{2}{|c|}{ Std Dev |Diff $\mid$ (Mod) } & 0.01072 & \multicolumn{2}{|c|}{ Std Dev [Diff] (Mod) } & 0.00971 & \multicolumn{2}{|c|}{ Std Dev |Diff| (Mod) } & 0.0151 \\
\hline
\end{tabular}

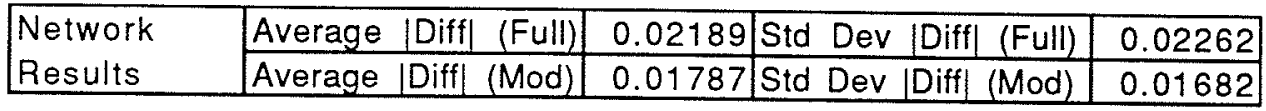


Training Results for V3.2.06.2

(Network 2.06.2, V3 Macro)

\begin{tabular}{|c|c|c|c|c|c|c|}
\hline \multirow[t]{2}{*}{ Destination } & \multicolumn{3}{|c|}{ Neuron o1 (Route 3) } & \multicolumn{3}{|c|}{ Neuron o2 (Route 6) } \\
\hline & Target & Meas & Diff & Target & Meas & Diff \\
\hline i1 & \multirow[t]{2}{*}{0.2569} & 0.31049 & -0.0536 & \multirow[t]{2}{*}{0.3986} & 0.33101 & 0.06759 \\
\hline 12 & & 0.34236 & -0.3424 & & 0.36397 & -0.364 \\
\hline i3 & 5 & 0.18644 & -0.0619 & 0.2663 & 0.20039 & 0.06591 \\
\hline ¡4 & 0.3 & 0. & -0 . & 0.3966 & 0.38 & 0.01598 \\
\hline i5 & 0.4146 & 0.4 & -0 & 0.4 & 0.4 & 0.0 \\
\hline 16 & 0.2335 & 0.7 & 0.0 & 0. & & \\
\hline ¡7 & 0.249 & 0.2 & -0.0 & 97 & 9 & \\
\hline 18 & 8 & 0.3 & -0 . & & 4 & \\
\hline i 9 & 0.46 & 0.4 & -0 . & & & \\
\hline i10 & 0.3524 & 0.2 & 0.05 & & & -0 \\
\hline$i$ & 0.3 & 99 & 0. & 0.3 & & \\
\hline$i 12$ & 0.47 & 0.47 & 0.0 & 0.4 & 0.6 & 12 \\
\hline$i 13$ & 5 & 0.6 & -0.0 & 0.659 & 0.6 & -0 \\
\hline$i 14$ & 5 & 0.3 & 0.0 & 0.3643 & 0.40 & -0.0431 \\
\hline$i 15$ & 0.4942 & 0.4 & & 0.4733 & 0.49 & -0.0222 \\
\hline$i 1$ & 0.6745 & 0.65827 & 0.0 & 0.6536 & 0.6753 & -0. \\
\hline \multirow{4}{*}{$\begin{array}{l}\text { Neuron } \\
\text { Results }\end{array}$} & \multicolumn{2}{|c|}{ Average |Diff| (Full) } & 0.0 & \multicolumn{2}{|c|}{ Average $\mid$ Diff $\mid$ (Full) } & 0.04 \\
\hline & \multirow{2}{*}{\multicolumn{2}{|c|}{\begin{tabular}{|l|l|} 
Average & Diff $\mid$ (Mod) \\
Std Dev & Diff $\mid$ (Full)
\end{tabular}}} & 0.02617 & \multicolumn{2}{|c|}{ Average |Diff] (Mod) } & \\
\hline & & & 0.08191 & Std Dev 1 & Diff] (Full) & 0.08663 \\
\hline & \multicolumn{2}{|c|}{ Std Dev |Diff| (Mod) } & 0.02223 & \multicolumn{2}{|c|}{ Std Dev $\mid$ Diff $\mid$ (Mod) } & 0.02087 \\
\hline \multirow{2}{*}{$\begin{array}{l}\text { Network } \\
\text { Results }\end{array}$} & \multirow{2}{*}{\multicolumn{2}{|c|}{\begin{tabular}{|l|l|l|} 
Average & Diff & (Full) \\
Average & Diff $\mid$ (Mod) \\
\end{tabular}}} & 0.0 & \multirow{2}{*}{\multicolumn{2}{|c|}{\begin{tabular}{|l|l|} 
Std Dev $\mid$ Diff $\mid$ (Full) \\
Std Dev $\mid$ Diff $\mid$ (Mod) \\
\end{tabular}}} & \\
\hline & & & 0.02657 & & & 0.0211 \\
\hline
\end{tabular}


Training Results for V3.2.08.2

(Network 2.08.8, V3 Macro)

\begin{tabular}{|c|c|c|c|c|c|c|}
\hline \multirow[t]{2}{*}{ Destination } & \multicolumn{3}{|c|}{ Neuron o1 (Route 3) } & \multicolumn{3}{|c|}{ Neuron o2 (Route 6) } \\
\hline & Target & Meas & Diff & Target & Meas & Diff \\
\hline$i 1$ & 0.2569 & 0.3107 & -0.0538 & 0.3986 & 0.33231 & 0.06629 \\
\hline i 2 & & 0.34226 & -0.3423 & & 0.36426 & -0.3643 \\
\hline i3 & 0.1245 & 0.18445 & -0.06 & 0.2663 & 0.20049 & 0.06581 \\
\hline$i 4$ & 0.3486 & 0.35909 & -0.0105 & 0.3966 & 0.38145 & 0.01515 \\
\hline i5 & 0.4146 & 0.44047 & -0.0259 & 0.4924 & 0.46309 & 0.02931 \\
\hline$i 6$ & 0.2335 & 0.18575 & 0.04775 & 0.1573 & 0.20162 & -0.0443 \\
\hline i 7 & 0.249 & 0.25662 & -0.0076 & 0.297 & 0.27662 & 0.02038 \\
\hline i 8 & 0.3588 & 0.36594 & -0.0071 & 0.4068 & 0.38819 & 0.01861 \\
\hline i 9 & 0.4618 & 0.47484 & -0.013 & 0.5097 & 0.49684 & 0.01286 \\
\hline i10 & 0.3524 & 0.29729 & 0.05511 & 0.2762 & 0.31842 & -0.0422 \\
\hline$i 11$ & 0.3588 & 0.34732 & 0.01148 & 0.3793 & 0.36972 & 0.00958 \\
\hline$i 12$ & 0.4776 & 0.4762 & 0.0014 & 0.4981 & 0.49844 & -0.0003 \\
\hline$i 13$ & 0.6385 & 0.64155 & -0.0031 & 0.659 & 0.65891 & $9 E-05$ \\
\hline $\mathrm{i} 14$ & 0.4405 & 0.38401 & 0.05649 & 0.3643 & 0.4061 & -0.0418 \\
\hline$i 15$ & 0.4942 & 0.47473 & 0.01947 & 0.4733 & 0.49571 & -0.0224 \\
\hline$i 16$ & 0.6745 & 0.65868 & 0.01582 & 0.6536 & 0.67354 & -0.0199 \\
\hline \multirow{4}{*}{$\begin{array}{l}\text { Neuron } \\
\text { Results }\end{array}$} & \multicolumn{2}{|c|}{ Average |Diff| (Full) } & 0.04567 & \multicolumn{2}{|c|}{ Average |Diff| (Full) } & 0.04834 \\
\hline & \multicolumn{2}{|c|}{ Average $\mid$ Diff $\mid$ (Mod) } & 0.0259 & \multicolumn{2}{|c|}{ Average |Diff| (Mod) } & 0.02727 \\
\hline & \multicolumn{2}{|c|}{ Std Dev |Diff $\mid$ (Full) } & 0.08189 & \multicolumn{2}{|c|}{ Std Dev JDiff $\mid$ (Full) } & 0.08661 \\
\hline & \multicolumn{2}{|c|}{ Std Dev $\mid$ Diff $\mid$ (Mod) } & 0.02198 & \multicolumn{2}{|c|}{ Std Dev |Diff $\mid$ (Mod) } & 0.02082 \\
\hline \multirow{2}{*}{$\begin{array}{l}\text { Network } \\
\text { Results } \\
\end{array}$} & \multirow{2}{*}{\multicolumn{2}{|c|}{ 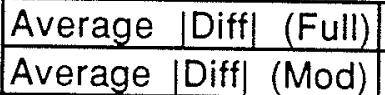 }} & 0.047 & \multirow{2}{*}{\multicolumn{2}{|c|}{\begin{tabular}{|l|l|} 
Std Dev |Diff $\mid$ (Full) \\
Std Dev |Diff $\mid$ (Mod)
\end{tabular}}} & 0.08293 \\
\hline & & & 0.02659 & & & 0.02105 \\
\hline
\end{tabular}


Training Results for V3.2.10.2

(Network 2.10.2, V3 Macro)

\begin{tabular}{|c|c|c|c|c|c|c|}
\hline \multirow[t]{2}{*}{ Destination } & \multicolumn{3}{|c|}{ Neuron o1 (Route 3 ) } & \multicolumn{3}{|c|}{ Neuron 02 (Route 6) } \\
\hline & Target & Meas & Diff & Target & Meas & Diff \\
\hline i1 & 0.2569 & 0.31285 & -0.056 & 0.3986 & 0.33566 & 0.06294 \\
\hline i 2 & & 0.33186 & -0.3319 & & 0.35402 & -0.354 \\
\hline i 3 & 0.1245 & 0.18222 & -0.0577 & 0.2663 & 0.19812 & 0.06818 \\
\hline ¡4 & 0.3486 & 0.35857 & -0.01 & 0.3966 & 0.38115 & 0.01545 \\
\hline i 5 & 0.4146 & 0.43791 & -0.0233 & 0.4924 & 0.46084 & 0.03156 \\
\hline i 6 & 0.2335 & 0.18441 & 0.04909 & 0.1573 & 0.20062 & -0.0433 \\
\hline$i 7$ & 0.249 & 0.25697 & -0.008 & 0.297 & 0.27713 & 987 \\
\hline i 8 & 0.3588 & 0.36222 & -0.0034 & 0.4068 & 0.38876 & 0.01804 \\
\hline i9 & 0.4618 & 0.47542 & -0.0136 & 0.5097 & 0.4974 & 123 \\
\hline $\mathrm{i10}$ & 0.3524 & 0.30009 & 0.05231 & 0.2762 & 0.32254 & -0.0463 \\
\hline $\mathbf{i 1 1}$ & 0.3588 & 0.34818 & 0.01062 & 0.3793 & 0.37104 & 0.00826 \\
\hline $\mathrm{i} 12$ & 0.4776 & 0.47472 & 0.00288 & 0.4981 & 0.49775 & 0.00035 \\
\hline i13 & 0.6385 & 0.64143 & -0.0029 & 0.659 & 0.6583 & 0.0007 \\
\hline ¡14 & 0.4405 & 0.37897 & 0.06153 & 0.3643 & 0.40217 & -0.0379 \\
\hline 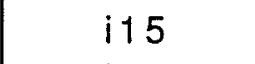 & 0.4942 & 0.47414 & 0.02006 & 0.4733 & 0.49433 & -0.021 \\
\hline$i 16$ & 0.6745 & 0.65897 & 0.01553 & 0.6536 & 0.67354 & -0.0199 \\
\hline \multirow{4}{*}{$\begin{array}{l}\text { Neuron } \\
\text { Results }\end{array}$} & \multicolumn{2}{|c|}{\begin{tabular}{|l|l|l|} 
Average $\mid$ Diff & (Full) \\
\end{tabular}} & 0.04492 & \multicolumn{2}{|c|}{ Average |Diff| (Full) } & 0.04751 \\
\hline & \multicolumn{2}{|c|}{ Average |Diff| (Mod) } & 0.02579 & \multicolumn{2}{|c|}{ Average |Diff $\mid$ (Mod) } & 0.02708 \\
\hline & \multicolumn{2}{|c|}{ Std Dev |Diff| (Full) } & 0.07955 & \multicolumn{2}{|c|}{ Std Dev |Diff| (Full) } & 0.08418 \\
\hline & \multicolumn{2}{|c|}{ Std Dev $\mid$ Diff $\mid$ (Mod) } & 0.02251 & \multicolumn{2}{|c|}{ Std Dev |Diff| (Mod) } & 0.02085 \\
\hline
\end{tabular}

\begin{tabular}{|l|l|l|l|l|l|l|}
\hline $\begin{array}{l}\text { Network } \\
\text { Results }\end{array}$ & Average & Diff $\mid$ (Full) & 0.04622 & Std Dev |Diff $\mid$ (Full) & 0.08058 \\
\cline { 2 - 6 } & Average & Diff $\mid$ (Mod) & 0.02644 & Std Dev & Diff $\mid$ (Mod) & 0.02133 \\
\hline
\end{tabular}


Training Results for V3.2.06.4

(Network 2.06.4, V3 Macro)

\begin{tabular}{|c|c|c|c|c|c|c|}
\hline \multirow[t]{2}{*}{ Destination } & \multicolumn{3}{|c|}{ Neuron o1 (Route 5) } & \multicolumn{3}{|c|}{ Neuron o2 (Route 8) } \\
\hline & Target & Meas & Diff & Target & Meas & $\overline{D i f f}$ \\
\hline$i 1$ & 0.3708 & 0.37876 & -0.008 & 0.3838 & 0.34064 & 0.04316 \\
\hline i2 & 0.6027 & 0.60405 & -0.0013 & 0.4619 & 0.45794 & 0.00396 \\
\hline i3 & 0.5031 & 0.50067 & 0.00243 & 0.3623 & 0.36096 & 0.00134 \\
\hline i4 & 0.3088 & 0.30585 & 0.00295 & 0.2418 & 0.25409 & -0.0123 \\
\hline i 5 & 0.213 & 0.26 & -0.0567 & 0.3376 & 0.27287 & 0.06473 \\
\hline i 6 & 0.5 & 0.54266 & 0.00544 & 0.3776 & 0.37932 & -0.0017 \\
\hline$i 7$ & 0.40 & 0.36134 & 0.04706 & 0.2379 & & -0.0238 \\
\hline ¡ 8 & 0.4225 & 0.32404 & 0.09846 & 0.1281 & 0.23097 & -0.1 \\
\hline i9 & & 0.37377 & -0.3738 & & 0.21279 & -0.2128 \\
\hline i10 & 0.6212 & 0.61504 & 0.00616 & 0.3 & 0.38052 & -0.0044 \\
\hline$i 11$ & 0.5181 & 0.49745 & 0.02065 & 0.273 & 0.2 & -0.0182 \\
\hline $\mathrm{i} 12$ & 0.5989 & 0.57 & 0.02 & 0.3045 & 0.30635 & -0.0019 \\
\hline$\lceil 13$ & 0.7597 & 0.7 & -0.0 & 0.4653 & 0.44776 & 0.01754 \\
\hline & & 0.6 & -0.0045 & 0.4179 & 0.40983 & 807 \\
\hline & 0.6 & 0.6 & -0.008 & 0.4085 & 0.38086 & 2764 \\
\hline$i 16$ & 0.8046 & 0.81807 & -0.0135 & 0.5102 & 0.49415 & 0.01605 \\
\hline \multirow{4}{*}{$\begin{array}{l}\text { Neuron } \\
\text { Results }\end{array}$} & \multicolumn{2}{|c|}{\begin{tabular}{|l|l|l|} 
Average & Diff & (Full) \\
\end{tabular}} & 0.04367 & \multicolumn{2}{|c|}{ Average |Diff| (Full) } & 0.03503 \\
\hline & \multicolumn{2}{|c|}{ Average |Diff| (Mod) } & 0.02167 & \multicolumn{2}{|c|}{ Average |Diff (Mod) } & 0.02318 \\
\hline & \multicolumn{2}{|c|}{ Std Dev |Diff $\mid$ (Full) } & 0.0918 & \multicolumn{2}{|c|}{ Std Dev |Diff $\mid$ (Full) } & 0.05463 \\
\hline & \multicolumn{2}{|c|}{ Std Dev |Diff| (Mod) } & 0.02698 & \multicolumn{2}{|c|}{ Std Dev |Diff| (Mod) } & 0.02811 \\
\hline
\end{tabular}

\begin{tabular}{|c|c|c|c|c|c|c|}
\hline \multirow[t]{2}{*}{ Destination } & \multicolumn{3}{|c|}{ Neuron o3 (Route 12) } & \multicolumn{3}{|c|}{ Neuron o4 (Route 13) } \\
\hline & Target & Meas & Diff & Target & Meas & Diff \\
\hline$i 1$ & 0.5985 & 0.59804 & 0.00046 & 0.8306 & 0.85096 & -0.0204 \\
\hline $\mathrm{i} 2$ & 0.6191 & 0.61865 & 0.00045 & 0.8512 & 0.84572 & 0.00548 \\
\hline i3 & 0.5195 & 0.505 & 0.0145 & 0.7516 & 0.75971 & -0.0081 \\
\hline i 4 & 0.4565 & 0.43128 & 0.02522 & 0.6886 & 0.69797 & -0.0094 \\
\hline i5 & 0.5523 & 0.54344 & 0.00886 & 0.7844 & 0.81463 & -0.0302 \\
\hline$i 6$ & 0.5072 & 0.49587 & 0.01133 & 0.7393 & 0.74216 & -0.0029 \\
\hline$i 7$ & 0.395 & 0.38042 & 0.01458 & 0.6271 & 0.62438 & 0.00272 \\
\hline$i 8$ & 0.3428 & 0.32835 & 0.01445 & 0.5749 & 0.55223 & 0.02267 \\
\hline$i 9$ & & 0.2227 & -0.2227 & & 0.36348 & -0.3635 \\
\hline$i 10$ & 0.3884 & 0.39599 & -0.0076 & 0.6205 & 0.60083 & 0.01967 \\
\hline$i 11$ & 0.2853 & 0.30311 & -0.0178 & 0.5174 & 0.48059 & 0.03681 \\
\hline$i 12$ & 0.1664 & 0.23377 & -0.0674 & 0.3985 & 0.32483 & 0.07367 \\
\hline$i 13$ & 0.3272 & 0.25269 & 0.07451 & 0.2377 & 0.28897 & -0.0513 \\
\hline ¡14 & 0.3851 & 0.3992 & -0.0141 & 0.6172 & 0.59413 & 0.02307 \\
\hline$i 15$ & 0.2762 & 0.32251 & -0.0463 & 0.5083 & 0.46754 & 0.04076 \\
\hline$i 16$ & 0.3712 & 0.3322 & 0.039 & 0.3929 & 0.43131 & -0.0384 \\
\hline \multirow{4}{*}{$\begin{array}{l}\text { Neuron } \\
\text { Results }\end{array}$} & \multicolumn{2}{|c|}{ Average |Diff (Full) } & 0.0362 & \multicolumn{2}{|c|}{\begin{tabular}{|l|l|l|} 
Average & Diff & (Full) \\
\end{tabular}} & 0.04681 \\
\hline & \multicolumn{2}{|c|}{ Average $\mid$ Diff $\mid$ (Mod) } & 0.02377 & \multicolumn{2}{|c|}{ Average |Diff| (Mod) } & 0.0257 \\
\hline & \multicolumn{2}{|c|}{ Std Dev |Diff $\mid$ (Full) } & 0.05442 & \multicolumn{2}{|c|}{ Std Dev $\mid$ Diff $\mid$ (Full) } & 0.08663 \\
\hline & \multicolumn{2}{|c|}{ Std Dev |Diff| (Mod) } & 0.02288 & \multicolumn{2}{|c|}{ Std Dev |Diff| (Mod) } & 0.02001 \\
\hline
\end{tabular}

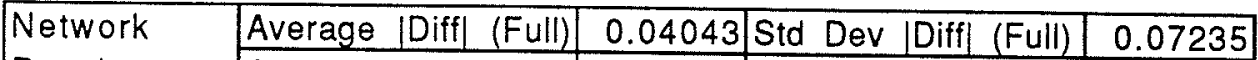

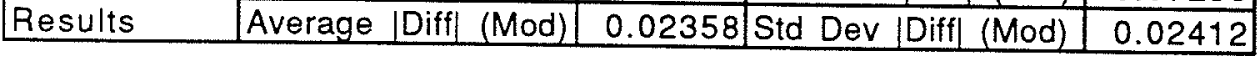


Training Results for V3.2.08.4

(Network 2.08.4, V3 Macro)

\begin{tabular}{|c|c|c|c|c|c|c|}
\hline \multirow[t]{2}{*}{ Destination } & \multicolumn{3}{|c|}{ Neuron o1 (Route 5) } & \multicolumn{3}{|c|}{ Neuron o2 (Route 8) } \\
\hline & Target & Meas & Diff & Target & Meas & Diff \\
\hline$i 1$ & 0.3708 & 0.36703 & 0.00377 & 0.3838 & 0.33978 & 0.04402 \\
\hline $\mathrm{i} 2$ & 0.6027 & 0.59848 & 0.00422 & 0.4619 & 0.45563 & 0.00627 \\
\hline i3 & 0.5031 & 0.49015 & 0.01295 & 0.3623 & 0.35799 & 0.00431 \\
\hline i 4 & 0.3088 & 0.292 & 0.01664 & 0.2418 & 0.24771 & -0.0059 \\
\hline i 5 & 0.213 & 0.2 & -0.0667 & 0.3376 & 0.28056 & 0.05704 \\
\hline i 6 & 0.5 & 0.5 & 0.01326 & 0.3776 & 0.37314 & 0.00446 \\
\hline i 7 & 0.4084 & 0.3 & 0.05567 & 0.2379 & 0.25 & -0.015 \\
\hline i 8 & 0.4225 & 0.31701 & 0.10549 & 0.1281 & 0.2219 & -0.0938 \\
\hline i9 & & 0.37403 & -0.374 & & 0.2342 & -0.2342 \\
\hline ¡10 & 0.6212 & 0.62331 & -0.0021 & 0.3 & 0.3755 & 0.0006 \\
\hline$\lceil 11$ & 0.5181 & 0.50364 & 0.01446 & 0.273 & 349 & -0.0119 \\
\hline$i 12$ & 0.5989 & 0.5 & 0.0 & 0.3045 & 0.29899 & 0.00551 \\
\hline$\lcm{13}$ & 0.7 & 0.7 & -0.0208 & 0.4653 & 0.45453 & 0.01077 \\
\hline$i 14$ & 63 & 0.67192 & -0.0089 & 0.4179 & 0.40 & 576 \\
\hline$\lceil 15$ & 0.6536 & 0.6534 & 0.0002 & 0.4085 & 0.3 & 3373 \\
\hline$i 16$ & 0.8046 & 0.8121 & -0.0075 & 0.5102 & 0.49482 & 0.01538 \\
\hline \multirow{4}{*}{$\begin{array}{l}\text { Neuron } \\
\text { Results }\end{array}$} & \multicolumn{2}{|c|}{ Average |Diff| (Full) } & 0.04577 & \multicolumn{2}{|c|}{ Average |Diff $\mid$ (Full) } & 0.03491 \\
\hline & \multicolumn{2}{|c|}{ Average Diff (Mod) } & 0.02388 & \multicolumn{2}{|c|}{ Average |Diff| (Mod) } & 0.02163 \\
\hline & \multicolumn{2}{|c|}{ Std Dev [Diff] (Full) } & 0.09207 & \multicolumn{2}{|c|}{ Std Dev |Diff| (Full) } & 0.05864 \\
\hline & \multicolumn{2}{|c|}{ Std Dev |Diff $\mid$ (Mod) } & 0.02953 & \multicolumn{2}{|c|}{ Std Dev [Diff] (Mod) } & 0.02566 \\
\hline
\end{tabular}

\begin{tabular}{|c|c|c|c|c|c|c|}
\hline \multirow[t]{2}{*}{ Destination } & \multicolumn{3}{|c|}{ Neuron o3 (Route 12) } & \multicolumn{3}{|c|}{ Neuron o4 (Route 13) } \\
\hline & Target & Meas & Diff & Target & Meas & Diff \\
\hline$i 1$ & 0.5985 & 0.59915 & -0.0006 & 0.8306 & 0.84719 & -0.0166 \\
\hline$i 2$ & 0.6191 & 0.61744 & 0.00166 & 0.8512 & 0.84278 & 0.00842 \\
\hline i3 & 0.5195 & 0.50367 & 0.01583 & 0.7516 & 0.75358 & -0.002 \\
\hline$i 4$ & 0.4565 & 0.42651 & 0.02999 & 0.6886 & 0.69321 & -0.0046 \\
\hline i5 & 0.5523 & 0.53994 & 0.01236 & 0.7844 & 0.80444 & -0.02 \\
\hline i 6 & 0.5072 & 0.49132 & 0.01588 & 0.7393 & 0.73716 & 0.00214 \\
\hline$i 7$ & 0.395 & 0.37019 & 0.02481 & 0.6271 & 0.61763 & 0.00947 \\
\hline i 8 & 0.3428 & 0.31556 & 0.02724 & 0.5749 & 0.5403 & 0.0346 \\
\hline i 9 & & 0.29107 & -0.2911 & & 0.49315 & -0.4932 \\
\hline$i 10$ & 0.3884 & 0.3829 & 0.0055 & 0.6205 & 0.58695 & 0.03355 \\
\hline i11 & 0.2853 & 0.2891 & -0.0038 & 0.5174 & 0.4662 & 0.0512 \\
\hline$i 12$ & 0.1664 & 0.23659 & -0.0702 & 0.3985 & 0.34665 & 0.05185 \\
\hline$i 13$ & 0.3272 & 0.26481 & 0.06239 & 0.2377 & 0.28451 & -0.0468 \\
\hline$i 14$ & 0.3851 & 0.38833 & -0.0032 & 0.6172 & 0.58684 & 0.03036 \\
\hline$i 15$ & 0.2762 & 0.32455 & -0.0484 & 0.5083 & 0.47633 & 0.03197 \\
\hline$i 16$ & 0.3712 & 0.33749 & 0.03371 & 0.3929 & 0.42664 & -0.0337 \\
\hline \multirow{4}{*}{$\begin{array}{l}\text { Neuron } \\
\text { Results }\end{array}$} & \multicolumn{2}{|c|}{ Average |Diff (Full) } & 0.04042 & \multicolumn{2}{|c|}{ Average |Diff| (Full) } & 0.05441 \\
\hline & \multicolumn{2}{|c|}{ Average |Diff $\mid$ (Mod) } & 0.02371 & \multicolumn{2}{|c|}{ Average Diffl (Mod) } & 0.02516 \\
\hline & \multirow{2}{*}{\multicolumn{2}{|c|}{ Std Dev |Diff $\mid$ (Full) }} & 0.07017 & \multirow{2}{*}{\multicolumn{2}{|c|}{\begin{tabular}{|l|} 
Std Dev |Diff| (Full) \\
Std Dev |Diff (Mod) (Mod
\end{tabular}}} & 0.11821 \\
\hline & & & 0.02209 & & & 0.01749 \\
\hline
\end{tabular}

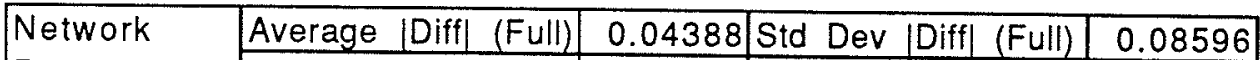
\begin{tabular}{|l|l|l|l|l|l|l|}
\cline { 2 - 5 } Results & Average & Diff & (Mod) & 0.02359 & Std Dev & Diff \\
(Mod) & 0.02352 \\
\hline
\end{tabular} 
Training Results for V3.2.10.4

(Network 2.10.4, V3 Macro)

\begin{tabular}{|c|c|c|c|c|c|c|}
\hline \multirow[t]{2}{*}{ Destination } & \multicolumn{3}{|c|}{ Neuron o1 (Route 5) } & \multicolumn{3}{|c|}{ Neuron o2 (Route 8) } \\
\hline & Target & Meas & Diff & Target & Meas & Diff \\
\hline$i 1$ & 0.3708 & 0.372 & -0.0012 & 0.3838 & 0.33996 & 0.04384 \\
\hline$i 2$ & 0.6027 & 0.60148 & 0.00122 & 0.4619 & 0.45605 & 0.00585 \\
\hline i3 & 0.5031 & 0.49533 & 0.00777 & 0.3623 & 0.35898 & 0.00332 \\
\hline i 4 & 0.3088 & 0.31182 & -0.003 & 0.2418 & 0.2548 & -0.013 \\
\hline i5 & 0.213 & 0.27762 & -0.0646 & 0.3376 & 0.28193 & 0.05567 \\
\hline i6 & 0.5481 & 0.53986 & 0.00824 & 0.3776 & 0.3736 & 0.004 \\
\hline i 7 & 0.4084 & 0.36558 & 0.04282 & 0.2379 & 0.25618 & -0.0183 \\
\hline$i 8$ & 0.4225 & 0.32402 & 0.09848 & 0.1281 & 0.22137 & -0.0933 \\
\hline i9 & & 0.443 & -0.443 & & 0.31402 & -0.314 \\
\hline 110 & 0.6212 & 0.61367 & 0.00753 & 0.3761 & 0.37371 & 0.00239 \\
\hline$i 11$ & 0.5181 & 0.49272 & 0.02538 & 0.273 & 0.28942 & -0.0164 \\
\hline$i 12$ & 0.5989 & 0.57665 & 0.02225 & 0.3045 & 0.3084 & -0.0039 \\
\hline 113 & 0.7597 & 0.78569 & -0.026 & 0.4653 & 0.45449 & 0.01081 \\
\hline$i 14$ & 0.663 & 0.66629 & -0.0033 & 0.4179 & 0.40131 & 0.01659 \\
\hline i15 & 0.6536 & 0.6536 & 0 & 0.4085 & 0.38058 & 0.02792 \\
\hline$i 16$ & 0.8046 & 0.81814 & -0.0135 & 0.5102 & 0.49975 & 0.01045 \\
\hline \multirow{4}{*}{$\begin{array}{l}\text { Neuron } \\
\text { Results }\end{array}$} & \multicolumn{2}{|c|}{ Average |Diff $\mid$ (Full) } & 0.04802 & \multicolumn{2}{|c|}{ Average |Diff| (Full) } & 0.03998 \\
\hline & \multicolumn{2}{|c|}{ Average $\mid$ Diff $\mid$ (Mod) } & 0.02169 & \multicolumn{2}{|c|}{ Average |Diff (Mod) } & 0.02171 \\
\hline & \multicolumn{2}{|c|}{ Std Dev |Diff $\mid$ (Full) } & 0.10871 & \multicolumn{2}{|c|}{ Std Dev [Diff| (Full) } & 0.07698 \\
\hline & \multicolumn{2}{|c|}{ Std Dev |Diff| (Mod) } & 0.02785 & \multicolumn{2}{|c|}{ Std Dev |Diff $\mid$ (Mod) } & 0.02505 \\
\hline
\end{tabular}

\begin{tabular}{|c|c|c|c|c|c|c|}
\hline \multirow[t]{2}{*}{ Destination } & \multicolumn{3}{|c|}{ Neuron o3 (Route 12) } & \multicolumn{3}{|c|}{ Neuron o4 (Route 13) } \\
\hline & Target & Meas & Diff & Target & Meas & Diff \\
\hline$i 1$ & 0.5985 & 0.59644 & 0.00206 & 0.8306 & 0.84772 & -0.0171 \\
\hline i 2 & 0.6191 & 0.61877 & 0.00033 & 0.8512 & 0.84486 & 0.000 \\
\hline i3 & 0.5195 & 0.50345 & 0.01605 & 0.7516 & 0.75345 & -0.001 \\
\hline i 4 & 0.4565 & 0.42226 & 0.03424 & 0.6886 & 0.687 & 0.0016 \\
\hline i 5 & 0.5523 & 0.54052 & 0.01178 & 0.7844 & 0.80542 & -0.021 \\
\hline i6 & 0.50 & 0.45 & 0.01624 & 0.7 & 0.73809 & 0.00121 \\
\hline$i 7$ & 0.5 & 0.36971 & 0.02529 & 0.6271 & 0.6 & 0.00884 \\
\hline i 8 & 0.3428 & 0.30647 & 0.03633 & 0.5 & 0.5 & 0.04789 \\
\hline i 9 & & 0.43965 & -0.4397 & & 0.69181 & -0.6918 \\
\hline i10 & 0.3884 & 0.38729 & 0.00111 & 0.6205 & 0.59039 & 0.0301 \\
\hline$i 11$ & 0.2853 & 0.30184 & -0.0165 & 0.5174 & 0.47867 & 0.03873 \\
\hline$\lceil 12$ & 0.1664 & 0.24764 & -0.0812 & 0.3 & 0.3 & 0.04476 \\
\hline$\lceil 13$ & 0.3272 & 0.2 & 0.06327 & 0.2 & 0.28 & -0.0517 \\
\hline$i 14$ & 0.3 & 0.3 & -0.0031 & 0.6172 & 0.58155 & 0.03565 \\
\hline$i 15$ & 0.2762 & 0.33204 & -0.0558 & 0.5083 & $0.4 \varepsilon$ & 2646 \\
\hline$\$ 16$ & 0.3712 & 0.34386 & 0.02734 & 0.3929 & 0.4397 & -0.0468 \\
\hline \multirow{4}{*}{$\begin{array}{l}\text { Neuron } \\
\text { Results }\end{array}$} & \multicolumn{2}{|c|}{ Average |Diff| (Full) } & 0.0519 & \multicolumn{2}{|c|}{ Average |Diff| (Full) } & 0.06699 \\
\hline & \multicolumn{2}{|c|}{ Average |Diff $\mid$ (Mod) } & 0.02605 & \multicolumn{2}{|c|}{ Average |Diff| (Mod) } & 0.02534 \\
\hline & \multicolumn{2}{|c|}{ Std Dev |Diff $\mid$ (Full) } & 0.10606 & \multicolumn{2}{|c|}{ Std Dev |Diff| (Full) } & 0.16757 \\
\hline & \multicolumn{2}{|c|}{ Std Dev |Diff (Mod) } & 0.02445 & \multicolumn{2}{|c|}{ Std Dev |Diff| (Mod) } & 0.01846 \\
\hline
\end{tabular}

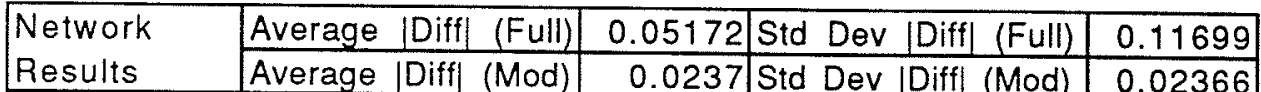


Training Results for V3.2.06.6

(Network 2.06.6, V3 Macro)

\begin{tabular}{|c|c|c|c|c|c|c|c|c|c|}
\hline \multirow[t]{2}{*}{ Destination } & \multicolumn{3}{|c|}{ Neuron o1 (Route 7) } & \multicolumn{3}{|c|}{ Neuron o2 (Route 8) } & \multicolumn{3}{|c|}{ Neuron o3 (Route 10) } \\
\hline & Target & Meas & Diff & Target & Meas & Diff & Target & Meas & Diff \\
\hline i 1 & 0.3763 & 0.31922 & 0.05708 & 0.4256 & 0.43095 & -0.0054 & 0.4882 & 0.45639 & 0.0318 \\
\hline i 2 & 0.3588 & 0.33716 & 0.02164 & 37 & 0.48239 & 0.02131 & 0.3793 & 0.35724 & 0.0220 \\
\hline i3 & 0.2592 & 0.2619 & -0.0027 & 0.4041 & 0.35279 & 0.05131 & 0.3559 & 0.3762 & -0.020 \\
\hline ¡ 4 & 0.2343 & 0.20681 & 0.02749 & 0.2 & 0. & 0.01802 & 0.4862 & 0.42071 & 0.0654 \\
\hline$i 5$ & 0.3301 & 0.32354 & 0.00656 & 0.5 & 0.8 & 0.04548 & 82 & 42 & -0.012 \\
\hline 16 & 0.2745 & 2885 & -0.014 & 0. & 0.4 & -0.0264 & 0.247 & 0.2 & 0 . \\
\hline i 7 & 0.13 & 0.1779 & -0.0431 & 0.2 & 0.22776 & 0.0 & & & \\
\hline i 8 & 0.2445 & 0.23781 & 0.00669 & 0.1 & 0.2 & -0 . & & 12 & \\
\hline i 9 & 0.3476 & 0.28712 & 0.06048 & 0.8 & 0.2 & 0.0 & & 12 & 0.0 \\
\hline 110 & 0.3933 & 0.32988 & 0.06342 & 0.5382 & 0.5 & 0.02328 & 0.1281 & 82 & -0 . \\
\hline$i 11$ & & 0.00575 & -0.0058 & & 0.00616 & -0.0062 & & 0.00 & -0 . \\
\hline$i 12$ & 0.4209 & 0.41683 & 0.00407 & 0.3 & 0.33455 & 0.01175 & 0.4713 & 0.4 & 0.0109 \\
\hline$i 13$ & 0.5603 & 0.55759 & 0.00271 & 0.4 & 32 & 0.0375 & 321 & 0.6 & -0.024 \\
\hline $\mathrm{i}$ & 0.4815 & 0.48 & 0.00 & 0.6331 & 0.55467 & 0.07843 & 0.2526 & 0.23058 & 0.0 \\
\hline & 07 & 49 & 0.01 & 0.456 & 0.44938 & 0.00662 & 0.3615 & 0.3 & -0 \\
\hline & 0.6266 & 0.63397 & -0.0074 & 0.552 & 0.59507 & -0.0 & 0.5418 & 0.4954 & 0.0464 \\
\hline \multirow{4}{*}{$\begin{array}{l}\text { Neuron } \\
\text { Results }\end{array}$} & \multicolumn{2}{|c|}{ Average |Diff| (Full) } & 0.02135 & \multicolumn{2}{|c|}{ Average |Diff| (Full) } & 09 & \multicolumn{2}{|c|}{ Average |Diff| (Full) } & 0.02574 \\
\hline & \multicolumn{2}{|c|}{ Average |Diff $\mid$ (Mod) } & 0.02239 & \multicolumn{2}{|c|}{ Average |Diff| (Mod) } & 0.03255 & \multicolumn{2}{|c|}{ Average [Diff| (Mod) } & 0.02713 \\
\hline & \multicolumn{2}{|c|}{ Std Dev |Diff| (Full) } & 0.02225 & \multicolumn{2}{|c|}{\begin{tabular}{|l|l|} 
Std Dev $\mid$ Diff $\mid$ (Full) \\
\end{tabular}} & 0.02113 & \multicolumn{2}{|c|}{ Std Dev [Diff] (Full) } & 0.01888 \\
\hline & \multicolumn{2}{|c|}{ Std Dev |Diff $\mid$ (Mod) } & 0.02263 & \multicolumn{2}{|c|}{ Std Dev [Diff] (Mod) } & 0.02077 & \multicolumn{2}{|c|}{ Std Dev $\mid$ Diff $\mid$ (Mod) } & 0.01868 \\
\hline
\end{tabular}

\begin{tabular}{|c|c|c|c|c|c|c|c|c|c|}
\hline \multirow[t]{2}{*}{ Destination } & \multicolumn{3}{|c|}{ Neuron o4 (Route 12) } & \multicolumn{3}{|c|}{ Neuron o5 (Route 14) } & \multicolumn{3}{|c|}{ Neuron o6 (Route 15) } \\
\hline & Target & Meas & Diff & Target & Meas & Diff & Target & Meas & Diff \\
\hline i1 & & 0.6009 & -0.025 & 0.6182 & .60927 & 0.00893 & 0.7023 & 0.65786 & 0.04444 \\
\hline i2 & 0.6541 & 0.60543 & 04867 & 0.5092 & & 0.00085 & 0.6088 & 0.60102 & 0.00778 \\
\hline i3 & 0.5545 & 67 & 6383 & 59 & & -0.0182 & 0.5854 & 0.54422 & 0.0411 \\
\hline i 4 & 0.4339 & & -0.0155 & 0.6162 & & 0.05826 & 03 & 07 & -0 \\
\hline i5 & 0.4732 & 87 & 0.03033 & 0.712 & 0.65924 & 0.05276 & 997 & 0.59318 & 0.0065 \\
\hline$i 6$ & 0.5696 & 0.5 & 0.04272 & 0.3 & 0 . & 0.04055 & 765 & 477 & \\
\hline i 7 & 0.43 & 0.3 & 0.03415 & 0.5166 & 36 & 0.0 & 64 & & 0. \\
\hline i 8 & 0.3203 & 0.27749 & 0.0 & 0.565 & 0.5 & 0.0 & 67 & 22 & \\
\hline 19 & 0.2853 & & 0. & 0.53 & 0. & -0 & 17 & 78 & -0 \\
\hline$i 10$ & 0.4871 & & -0 & 0.29 & 0.2 & 0.04812 & 0.3939 & 0.3 & \\
\hline i11 & & 0.0 & -0. & & 0.0 & -0.0048 & & & \\
\hline$i 12$ & 0.1439 & $0.1 \varepsilon$ & -0.0411 & 0.3886 & 0.3 & 0.0 & 0.2 & 674 & 0.0335 \\
\hline i13 & 0.3047 & 0.2705 & 0.0342 & 0.5494 & 0.5 & -0. & 11 & 701 & 0.06 \\
\hline i14 & 0.3626 & 0.36536 & -0.0028 & 0.1699 & 0.2 & -0.0 & 95 & 0.27221 & -0.002 \\
\hline i15 & 0.2536 & 0.1 & 0.0 & 0.2789 & 0.2 & 0.0 & 05 & 0.18856 & -0.028 \\
\hline$i 1$ & 0.3496 & 0.32807 & 0.0 & 0.4591 & 0.40409 & 0.05501 & 0.3408 & 0.3183 & 0.0225 \\
\hline \multirow{4}{*}{$\begin{array}{l}\text { Neuron } \\
\text { Results }\end{array}$} & \multicolumn{2}{|c|}{ Average $\mid$ Diff $\mid$ (Full) } & 0.03151 & \multicolumn{2}{|c|}{ Average |Diff| (FulI) } & 0.02911 & \multicolumn{2}{|c|}{ Average |Diff (Full) } & \\
\hline & \multicolumn{2}{|c|}{\begin{tabular}{|l|l|} 
Average $\mid$ Diff $\mid$ (Mod) \\
\end{tabular}} & 0.03324 & \multicolumn{2}{|c|}{ Average $\mid$ Diff $\mid$ (Mod) } & 0.03072 & \multicolumn{2}{|c|}{ Average |Diff| (Mod) } & \\
\hline & \multirow{2}{*}{\multicolumn{2}{|c|}{$\frac{\text { Std Dev } \mid \text { Diff } \mid \text { (Full) }}{\text { Std Dev [Diff] (Mod) }}$}} & 0.01884 & \multicolumn{2}{|c|}{ Std Dev |Diff| (Full) } & 0.02232 & \multirow{2}{*}{\multicolumn{2}{|c|}{ Std Dev [Diff $\mid$ (Full) }} & 0.01862 \\
\hline & & & 0.01813 & Std Dev $\mid \mathrm{D}$ & iff $\mid$ (Mod) & 0.02212 & & iff (Mod) & 0.01868 \\
\hline
\end{tabular}

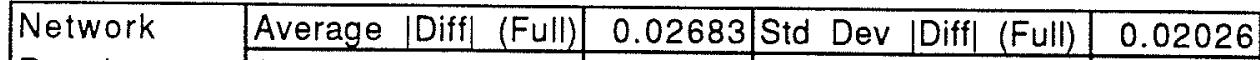

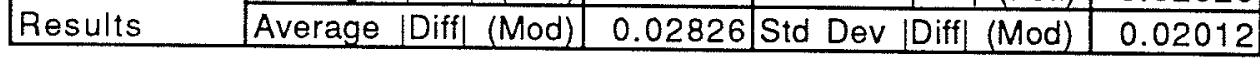


Training Results for V3.2.08.6

(Network 2.08.6, V3 Macro)

\begin{tabular}{|c|c|c|c|c|c|c|c|c|c|}
\hline \multirow[t]{2}{*}{ Destination } & \multicolumn{3}{|c|}{ Neuron o1 (Route 7) } & \multicolumn{3}{|c|}{ Neuron 02 (Route 8) } & \multicolumn{3}{|c|}{ Neuron 03 (Route 10) } \\
\hline & Target & Meas & Diff & Target & Meas & Diff & Target & Meas & Diff \\
\hline$i 1$ & 0.3763 & 0.33826 & 0.03804 & 0.4256 & 0.44574 & -0.0201 & 0.4882 & 0.48056 & 0.00764 \\
\hline i2 & 0.3588 & 0.34062 & 0.01818 & 0.5037 & 0.51702 & -0.0133 & 0.3793 & 0.33204 & 0.04726 \\
\hline i3 & 0.2592 & 0.27116 & -0.012 & 0.4041 & 0.39294 & 0.01116 & 0.3559 & 0.33939 & 0.01651 \\
\hline ¡ 4 & 0.2343 & 0.22674 & 0.00756 & 0.2836 & 0.26405 & 0.01955 & 0.4862 & 0.48497 & 0.00123 \\
\hline ¡5 & 0.3301 & 0.35087 & -0.0208 & 0.3794 & 0.36476 & 0.01464 & 0.582 & 0.59931 & -0.0173 \\
\hline 16 & 0.2745 & 0.26325 & 0.01125 & 0.4194 & 0.40648 & 0.01292 & 0.247 & 0.25037 & -0.0 .034 \\
\hline i 7 & 0.1348 & 0.16411 & -0.0293 & 0.2797 & 0.21672 & 0.06298 & 0.3867 & 0.38461 & 0.00209 \\
\hline i8 & 0.2445 & 0.20613 & 0.03837 & 0.1699 & 0.19544 & -0.0255 & 0.4964 & 0.50233 & -0.0059 \\
\hline i9 & 0.3476 & 0.34014 & 0.00746 & 0.273 & 0.28671 & -0.0137 & 0.5995 & 0.56238 & 0.03712 \\
\hline i10 & 0.3933 & 0.36568 & 0.02762 & 0.5382 & 0.5221 & 0.0161 & 0.1281 & 0.21 & \\
\hline$i 11$ & & 0.11599 & -0.116 & & 0.1386 & -0.1386 & & 0.0 & \\
\hline 112 & 0.4209 & 0.42675 & -0.0059 & 0.3463 & 0.32745 & 0.01885 & 0.4713 & 047169 & \\
\hline 113 & 0.5603 & 0.55488 & 0.00542 & 0.4857 & 047261 & 0.01309 & & & \\
\hline$i 14$ & 0.4815 & 0.50035 & -0.0189 & 0.6331 & 0.63312 & $-2 F-05$ & & & 2 \\
\hline$i 15$ & 0.5307 & 0.52981 & 0.00089 & 0456 & 045380 & 0 & & 0.17913 & 0.07347 \\
\hline$¡ 16$ & 0.6266 & 061528 & & & & 0.0 & 0.3615 & 0.36575 & -0.0043 \\
\hline \multirow{5}{*}{$\begin{array}{l}\text { Neuron } \\
\text { Results }\end{array}$} & \multicolumn{2}{|c|}{ Average |Diff| (Full) } & 0.011132 & 0.552 & 0.54988 & 0.00212 & 0.5418 & 0.54503 & -0.0032 \\
\hline & \multirow{2}{*}{\multicolumn{2}{|c|}{ Average |Diff $\mid$ (Mod) }} & 0.0 & \multicolumn{2}{|c|}{ Average |Diff| (Full) } & 0.02405 & \multicolumn{2}{|c|}{\begin{tabular}{|l|l|} 
Average $\mid$ Diff $\mid$ (Full) \\
\end{tabular}} & 0.02573 \\
\hline & Std Dev |Diff $\mid$ (Full) & & 0.01686 & \multicolumn{2}{|c|}{ Average |Diff| (Mod) } & 0.01642 & \multicolumn{2}{|c|}{ Average |Diff (Mod) } & 0.02098 \\
\hline & \multirow{2}{*}{\multicolumn{2}{|c|}{ Std Dev |Diff| (Mod) }} & 0.02731 & \multicolumn{2}{|c|}{ Std Dev |Diff| (Full) } & 0.03369 & \multicolumn{2}{|c|}{ Std Dev |Diff| (Full) } & 0.03248 \\
\hline & & & 0.01187 & \multicolumn{2}{|c|}{ Std Dev |Diff| (Mod) } & 0.01471 & \multicolumn{2}{|c|}{ Std Dev |Diff] (Mod) } & 0.02724 \\
\hline
\end{tabular}

\begin{tabular}{|c|c|c|c|c|c|c|c|c|c|}
\hline \multirow[t]{2}{*}{ Destination } & \multicolumn{3}{|c|}{ Neuron o4 (Route 12) } & \multicolumn{3}{|c|}{ Neuron o5 (Route 14) } & \multicolumn{3}{|c|}{ Neuron o6 (Route 15) } \\
\hline & Target & Meas & Diff & Target & Meas & Diff & Target & Meas & Diff \\
\hline$i 1$ & 0.5759 & 0.6062 & -0.0303 & 0.6182 & 0.62102 & -0.0028 & 0.7023 & 0.66179 & 0.04051 \\
\hline$i 2$ & 0.6541 & 0.65765 & -0.0035 & 0.5092 & 0.49548 & 0.01372 & 0.6088 & 0.62986 & -0.0211 \\
\hline i3 & 0.5545 & 0.55498 & -0.0005 & 0.4859 & 0.48892 & -0.003 & 0.5854 & 0.57528 & 0.01012 \\
\hline ¡ 4 & 0.4339 & 0.4299 & 0.004 & 0.6162 & 0.59975 & 0.01645 & 0.5603 & 0.56584 & -0.0055 \\
\hline i 5 & 0.4732 & 0.46778 & 0.00542 & 0.712 & 0.66879 & 0.04321 & 0.5997 & 0.60398 & -0.0043 \\
\hline$i 6$ & 0.5696 & 0.5337 & 0.0359 & 0.3769 & 0.38075 & -0.0038 & 0.4765 & 0.50441 & -0.0279 \\
\hline$i 7$ & 0.43 & 0.41821 & 0.01179 & 0.5166 & 0.53277 & -0.0162 & 0.5564 & 0.53868 & 0.01772 \\
\hline ¡ 8 & 0.3203 & 0.28696 & 0.03334 & 0.565 & 0.55946 & 0.00554 & 0.4467 & 0.44863 & -0.0019 \\
\hline ¡ 9 & 0.2853 & 0.27719 & 0.00811 & 0.53 & 0.5528 & -0.0228 & 0.4117 & 0.40852 & 0.00318 \\
\hline$¡ 10$ & 0.4871 & 0.48462 & 0.00248 & 0.2944 & 0.27258 & 0.02182 & 0.3939 & 0.38768 & 0.00622 \\
\hline$i 11$ & & 0.13178 & -0.1318 & & 0.11081 & -0.1108 & & 0.12497 & -0.125 \\
\hline ¡12 & 0.1439 & 0.18246 & -0.0386 & 0.3886 & 0.37619 & 0.01241 & 0.2703 & 0.23701 & 0.03329 \\
\hline$i 13$ & 0.3047 & 0.32384 & -0.0191 & 0.5494 & 0.55918 & -0.0098 & 0.4311 & 0.4123 & 0.0188 \\
\hline ¡14 & 0.3626 & 0.36953 & -0.0069 & 0.1699 & 0.16066 & 0.00924 & 0.2695 & 0.22683 & 0.04267 \\
\hline$i 15$ & 0.2536 & 0.18528 & 0.06832 & 0.2789 & 0.25163 & 0.02727 & 0.1605 & 0.17521 & -0.0147 \\
\hline i16 & 0.3496 & 0.31869 & 0.03091 & 0.4591 & 0.45636 & 0.00274 & 0.3408 & 0.34546 & -0.0047 \\
\hline \multirow{4}{*}{$\begin{array}{l}\text { Neuron } \\
\text { Results }\end{array}$} & \multicolumn{2}{|c|}{ Average |Diff| (Full) } & 0.02694 & \multicolumn{2}{|c|}{\begin{tabular}{|l|l|l|} 
Average & Diff & (Full) \\
\end{tabular}} & 0.0201 & \multicolumn{2}{|c|}{ Average 1 Diff (Full) } & 0.0236 \\
\hline & \multicolumn{2}{|c|}{ Average |Diff (Mod) } & 0.01995 & \multicolumn{2}{|c|}{ Average [Diff| (Mod) } & 0.01406 & \multicolumn{2}{|c|}{ Average IDiff (Mod) } & 0.01684 \\
\hline & \multicolumn{2}{|c|}{ Std Dev |Diff $\mid$ (Full) } & 0.03353 & \multicolumn{2}{|c|}{ Std Dev |Diff $\mid$ (Fuli) } & 0.02651 & \multicolumn{2}{|c|}{\begin{tabular}{|l|l|l|} 
Std Dev |Diff & (Full) \\
\end{tabular}} & 0.03012 \\
\hline & \multicolumn{2}{|c|}{ Std Dev |Diff (Mod) } & 0.01917 & \multicolumn{2}{|c|}{ Std Dev |Diff| (Mod) } & 0.01124 & \multicolumn{2}{|c|}{ Std Dev |Diff (Mod) } & 0.01375 \\
\hline
\end{tabular}

\begin{tabular}{|l|l|l|l|l|r|}
\hline $\begin{array}{l}\text { Network } \\
\text { Results }\end{array}$ & Average $\mid$ Diff $\mid$ (Full) & 0.02391 & Std Dev $\mid$ Diff $\mid$ (Full) & 0.03 \\
\cline { 2 - 6 } & Average $\mid$ Diff $\mid$ (Mod) & 0.01752 & Std Dev Diff $\mid$ (Mod) & 0.0169 \\
\hline
\end{tabular}


Training Results for V3.2.10.6

(Network 2.10.6, V3 Macro)

\begin{tabular}{|c|c|c|c|c|c|c|c|c|c|}
\hline \multirow[t]{2}{*}{ Destination } & \multicolumn{3}{|c|}{ Neuron 01 (Route 7) } & \multicolumn{3}{|c|}{ Neuron o2 (Route 8 ) } & \multicolumn{3}{|c|}{ Neuron o3 (Route 10) } \\
\hline & Target & Meas & Diff & Target & Meas & Dו1ו & Target & Meas & Diff \\
\hline$i 1$ & 0.3763 & 0.33644 & 0.03986 & 0.4256 & 0.45935 & -0.0338 & 0.4882 & 0.44626 & 0.0419 \\
\hline i 2 & 0.3588 & 0.33784 & 0.02096 & 0.5037 & 0.51053 & -0.0068 & 0.3793 & 0.32306 & 0.0562 \\
\hline i3 & 0.2592 & 0.26823 & -0.009 & 0.4041 & 0.38779 & 0.01631 & 0.3559 & 0.33558 & 0.02032 \\
\hline ¡ 4 & 0.2343 & 393 & 0.02037 & 336 & 0.25529 & 0.02831 & 62 & 0.4 & $-0.002 \varepsilon$ \\
\hline i5 & 0.3 & 81 & -0.0217 & 94 & 0.3 & 0.02066 & 0.582 & 0.6 & -0.03 \\
\hline i6 & 0.2 & 0.26123 & 0.01327 & 0.4 & 0.3 & 0.02745 & 0.247 & 77 & \\
\hline i 7 & 0.1348 & 0.14953 & -0.0147 & 0.2797 & 0.19979 & 0.07991 & 0.3867 & 0.3 & 102 \\
\hline i 8 & 0.2445 & 0.18676 & 0.05774 & 0.1699 & 0.1943 & -0.0244 & 64 & 0. & 972 \\
\hline i9 & 0.3476 & 0.33046 & 0.01714 & 0.273 & 0.2 & -0 & & 0.5 & 174 \\
\hline$i 10$ & 0.3933 & 0.38857 & 0.00473 & 0.5382 & 0.5 & 0.00846 & 0.1281 & 0.2 & -0.0943 \\
\hline$i 11$ & & 0.06958 & -0 & & 0.0 & -0.0782 & & 0.07298 & -0.073 \\
\hline$i 12$ & & 0.4 & -0.0 & 3 & 0.3 & 0.02776 & & 0.4 & 05 \\
\hline & & 99 & 0.0104 & 0.4857 & & & & & 48 \\
\hline$\lceil 14$ & 0.4815 & 0.49048 & -0.009 & 0.6331 & 0.6 & -0 . & 26 & 0.1 & 0.0 \\
\hline ¡15 & 0.5307 & 0.51821 & 0.01249 & 0.456 & 0.4 & -0 & 15 & 0.3 & 0.0 \\
\hline$\lceil 16$ & 0.6266 & 0.62188 & 0.00472 & 0.552 & 0.55243 & -0.0004 & 0.5418 & 0.54533 & -0 \\
\hline \multirow{4}{*}{$\begin{array}{l}\text { Neuron } \\
\text { Results }\end{array}$} & \multicolumn{2}{|c|}{ Average |Diff| (Full) } & 0.02207 & \multicolumn{2}{|c|}{ Average |Diff| (Full) } & 0.02496 & \multicolumn{2}{|c|}{\begin{tabular}{l|l} 
Average & Diff \\
(Full)
\end{tabular}} & 0.03226 \\
\hline & \multicolumn{2}{|c|}{ Average |Diff (Mod) } & 0.0189 & \multicolumn{2}{|c|}{ Average |Diff (Mod) } & 0.02141 & \multicolumn{2}{|c|}{ Average |Diff| (Mod) } & 0.0 \\
\hline & \multirow{2}{*}{\multicolumn{2}{|c|}{\begin{tabular}{|l|l|l|} 
Std Dev |Diff| (Full) \\
Std
\end{tabular}}} & 0.01863 & \multicolumn{2}{|c|}{ Std Dev $\mid$ Diff $\mid$ (Full) } & 0.0234 & \multicolumn{2}{|c|}{ Std Dev $\mid$ Diff $\mid$ (Full) } & 0.0 \\
\hline & & Std Dev |Diff| (Mod) & 0.01413 & \multicolumn{2}{|c|}{ Std Dev |Diff| (Mod) } & 0.01926 & \multicolumn{2}{|c|}{\begin{tabular}{|l|l|l|} 
Std Dev & Diff & (Mod) \\
\end{tabular}} & 0.03086 \\
\hline
\end{tabular}

\begin{tabular}{|c|c|c|c|c|c|c|c|c|c|}
\hline \multirow[t]{2}{*}{ Destination } & \multicolumn{3}{|c|}{ Neuron o4 (Route 12) } & \multicolumn{3}{|c|}{ Neuron o5 (Route 14) } & \multicolumn{3}{|c|}{ Neuron o6 (Route 15) } \\
\hline & Target & Meas & Diff & Target & Meas & Diff & Target & Meas & Diff \\
\hline$i 1$ & 0.5759 & 0.63153 & -0.0556 & 0.6182 & 0.59779 & 0.02041 & 0.7023 & 0.66406 & 0.03824 \\
\hline$i 2$ & 0.6541 & 0.66547 & -0.0114 & 0.5092 & 0.48783 & 0.02137 & 0.6088 & 0.63268 & -0.0239 \\
\hline i 3 & 0.5545 & 0.55919 & -0.0047 & 0.4859 & 0.48394 & 0.00196 & 0.5854 & 0.57441 & 0.01099 \\
\hline ¡ 4 & 0.4339 & 0.4273 & 0.0066 & 0.6162 & 0.60361 & 0.01259 & 0.5603 & 0.5642 & -0.0039 \\
\hline i5 & 0.4732 & 0.44591 & 0.02729 & 0.712 & 0.67755 & 0.03445 & 0.5997 & 0.58885 & 0.01085 \\
\hline i 6 & 0.5696 & 0.52914 & 0.04046 & 0.3769 & 0.39143 & -0.0145 & 0.4765 & 0.50729 & -0.0308 \\
\hline 17 & 0.43 & 0.40739 & 0.02261 & 0.5166 & 0.53826 & -0.0217 & 0.5564 & 0.53608 & 0.02032 \\
\hline i 8 & 0.3203 & 0.2999 & 0.0204 & 0.565 & 0.55301 & 0.01199 & 0.4467 & 0.45658 & -0.0099 \\
\hline i9 & 0.2853 & 0.28803 & -0.0027 & 0.53 & 0.54779 & -0.0178 & 0.4117 & 0.41325 & -0.0016 \\
\hline$i 10$ & 0.4871 & 0.46566 & 0.02144 & 0.2944 & 0.26836 & 0.02604 & 0.3939 & 0.37294 & 0.02096 \\
\hline i11 & & 0.08085 & -0.0809 & & 0.08283 & -0.0828 & & 0.0842 & -0.0842 \\
\hline$i 12$ & 0.1439 & 0.15333 & -0.0094 & 0.3886 & 0.36244 & 0.02616 & 0.2703 & 0.20533 & 0.06497 \\
\hline$i 13$ & 0.3047 & 0.31194 & -0.0072 & 0.5494 & 0.56657 & -0.0172 & 0.4311 & 0.40554 & 0.02556 \\
\hline$i 14$ & 0.3626 & 0.38109 & -0.0185 & 0.1699 & 0.15014 & 0.01976 & 0.2695 & 0.23487 & 0.03463 \\
\hline$i 15$ & 0.2536 & 0.20661 & 0.04699 & 0.2789 & 0.26353 & 0.01537 & 0.1605 & 0.19898 & -0.0385 \\
\hline$i 16$ & 0.3496 & 0.30646 & 0.04314 & 0.4591 & 0.45276 & 0.00634 & 0.3408 & 0.33594 & 0.00486 \\
\hline \multirow{4}{*}{$\begin{array}{l}\text { Neuron } \\
\text { Results }\end{array}$} & \multicolumn{2}{|c|}{ Average |Diff (Full) } & 0.02621 & \multicolumn{2}{|c|}{\begin{tabular}{|l|l|} 
Average $\mid$ Diff $\mid$ (Full $\mid$ \\
\end{tabular}} & 0.0219 & \multicolumn{2}{|c|}{ 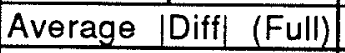 } & 0.0265 \\
\hline & \multicolumn{2}{|c|}{ Average $\mid$ Diff $\mid$ (Mod) } & 0.02257 & \multicolumn{2}{|c|}{ Average |Diff| (Mod) } & 0.01784 & \multicolumn{2}{|c|}{ Average [Diff (Mod) } & 0.02266 \\
\hline & \multicolumn{2}{|c|}{ Std Dev |Diff| (Full) } & 0.02185 & \multicolumn{2}{|c|}{ Std Dev |Diff| (Full) } & 0.01802 & \multicolumn{2}{|c|}{ Std Dev |Diff $\mid$ (Full) } & 0.02253 \\
\hline & \multicolumn{2}{|c|}{ Std Dev [Diff] (Mod) } & 0.01686 & \multicolumn{2}{|c|}{ Std Dev |Diff $\mid$ (Mod) } & 0.00808 & \multicolumn{2}{|c|}{ Std Dev |Diff $\mid$ (Mod) } & 0.01704 \\
\hline
\end{tabular}

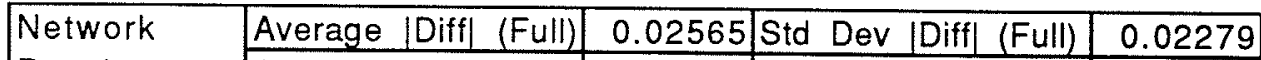

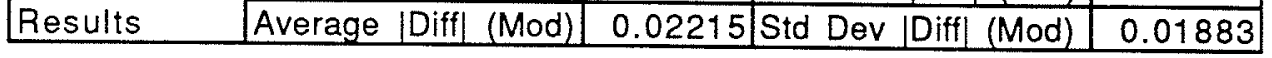


Pattern File for Node 2 of the Sample Network Modified training set

\begin{tabular}{|c|c|c|c|c|c|c|c|c|c|c|c|c|c|c|c|c|c|c|}
\hline \multirow{2}{*}{$\begin{array}{c}\text { Destination } \\
\text { Node }\end{array}$} & \multicolumn{16}{|c|}{ Input Vector } & \multicolumn{2}{|c|}{ Output Vector } \\
\hline & $i 1$ & $i 2$ & $i 3$ & $i 4$ & i5 & i 6 & $i 7$ & $i 8$ & i9 & $i 10$ & $i 11$ & $i 12$ & $i .13$ & $i 14$ & $i 15$ & $i 16$ & 01 & 02 \\
\hline$i 1$ & 1 & 0 & 0 & 0 & 0 & 0 & 0 & 0 & 0 & 0 & 0 & 0 & 0 & 0 & 0 & 0 & 0.25 & 0.75 \\
\hline$i 2$ & 0 & 1 & 0 & 0 & 0 & 0 & 0 & 0 & 0 & 0 & 0 & 0 & 0 & 0 & 0 & 0 & & \\
\hline$i 3$ & 0 & 0 & 1 & 0 & 0 & 0 & 0 & 0 & 0 & 0 & 0 & 0 & 0 & 0 & 0 & 0 & 0.25 & 0.75 \\
\hline$i 4$ & 0 & 0 & 0 & 1 & 0 & 0 & 0 & 0 & 0 & 0 & 0 & 0 & 0 & 0 & 0 & 0 & 0.25 & 0.75 \\
\hline$i 5$ & 0 & 0 & 0 & 0 & 1 & 0 & 0 & 0 & 0 & 0 & 0 & 0 & 0 & 0 & 0 & 0 & 0.25 & 0.75 \\
\hline$i 6$ & 0 & 0 & 0 & 0 & 0 & 1 & 0 & 0 & 0 & 0 & 0 & 0 & 0 & 0 & 0 & 0 & 0.75 & 0.25 \\
\hline$i 7$ & 0 & 0 & 0 & 0 & 0 & 0 & 1 & 0 & 0 & 0 & 0 & 0 & 0 & 0 & 0 & 0 & 0.25 & 0.75 \\
\hline$i 8$ & 0 & 0 & 0 & 0 & 0 & 0 & 0 & 1 & 0 & 0 & 0 & 0 & 0 & 0 & 0 & 0 & 0.25 & 0.75 \\
\hline i9 & 0 & 0 & 0 & 0 & 0 & 0 & 0 & 0 & 1 & 0 & 0 & 0 & 0 & 0 & 0 & 0 & 0.25 & 0.75 \\
\hline$i 10$ & 0 & 0 & 0 & 0 & 0 & 0 & 0 & 0 & 0 & 1 & 0 & 0 & 0 & 0 & 0 & 0 & 0.75 & 0.25 \\
\hline$i 11$ & 0 & 0 & 0 & 0 & 0 & 0 & 0 & 0 & 0 & 0 & 1 & 0 & 0 & 0 & 0 & 0 & 0.25 & 0.75 \\
\hline $\mathrm{i} 12$ & 0 & 0 & 0 & 0 & 0 & 0 & 0 & 0 & 0 & 0 & 0 & 1 & 0 & 0 & 0 & 0 & 0.25 & 0.75 \\
\hline$i 13$ & 0 & 0 & 0 & 0 & 0 & 0 & 0 & 0 & 0 & 0 & 0 & 0 & 1 & 0 & 0 & 0 & 0.25 & 0.75 \\
\hline 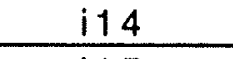 & 0 & 0 & 0 & 0 & 0 & 0 & 0 & 0 & 0 & 0 & 0 & 0 & 0 & 1 & 0 & 0 & 0.75 & 0.25 \\
\hline$i 15$ & 0 & 0 & 0 & 0 & 0 & 0 & 0 & 0 & 0 & 0 & 0 & 0 & 0 & 0 & 1 & 0 & 0.75 & 0.25 \\
\hline$i 16$ & 0 & 0 & 0 & 0 & 0 & 0 & 0 & 0 & 0 & 0 & 0 & 0 & 0 & 0 & 0 & 1 & 0.75 & 0.25 \\
\hline
\end{tabular}

Page B.28 
Pattern File for Node 9 of the Sample Network Modified training set

\begin{tabular}{|c|c|c|c|c|c|c|c|c|c|c|c|c|c|c|c|c|c|c|c|c|}
\hline \multirow{2}{*}{$\begin{array}{c}\text { Destination } \\
\text { Node }\end{array}$} & \multicolumn{16}{|c|}{ Input Vector } & \multicolumn{4}{|c|}{ Output Vector } \\
\hline & $i 1$ & $i 2$ & i 3 & $i 4$ & $i 5$ & i 6 & $i 7$ & i 8 & i9 & $i 10$ & $i 11$ & $i 12$ & $i 13$ & $i 14$ & $i 15$ & $i 16$ & 01 & 02 & 03 & 04 \\
\hline i1 & 1 & 0 & 0 & 0 & 0 & 0 & 0 & 0 & 0 & 0 & 0 & 0 & 0 & 0 & 0 & 0 & 0.125 & 0.375 & 0.625 & 0.875 \\
\hline$i 2$ & 0 & 1 & 0 & 0 & 0 & 0 & 0 & 0 & 0 & 0 & 0 & 0 & 0 & 0 & 0 & 0 & 0.375 & 0.125 & 0.625 & 0.875 \\
\hline 13 & 0 & 0 & 1 & 0 & 0 & 0 & 0 & 0 & 0 & 0 & 0 & 0 & 0 & 0 & 0 & 0 & 0.375 & 0.125 & 0.625 & 0.875 \\
\hline$i 4$ & 0 & 0 & 0 & 1 & 0 & 0 & 0 & 0 & 0 & 0 & 0 & 0 & 0 & 0 & 0 & 0 & 0.375 & 0.125 & 0.625 & 0.875 \\
\hline$i 5$ & 0 & 0 & 0 & 0 & 1 & 0 & 0 & 0 & 0 & 0 & 0 & 0 & 0 & 0 & 0 & 0 & 0.125 & 0.375 & 0.625 & 0.875 \\
\hline$i 6$ & 0 & 0 & 0 & 0 & 0 & 1 & 0 & 0 & 0 & 0 & 0 & 0 & 0 & 0 & 0 & 0 & 0.625 & 0.125 & 0.375 & 0.875 \\
\hline$i 7$ & 0 & 0 & 0 & 0 & 0 & 0 & 1 & 0 & 0 & 0 & 0 & 0 & 0 & 0 & 0 & 0 & 0.625 & 0.125 & 0.375 & 0.875 \\
\hline i 8 & 0 & 0 & 0 & 0 & 0 & 0 & 0 & 1 & 0 & 0 & 0 & 0 & 0 & 0 & 0 & 0 & 0.625 & 0.125 & 0.375 & 0.875 \\
\hline i9 & 0 & 0 & 0 & 0 & 0 & 0 & 0 & 0 & 1 & 0 & 0 & 0 & 0 & 0 & 0 & 0 & & & & \\
\hline$i 10$ & 0 & 0 & 0 & 0 & 0 & 0 & 0 & 0 & 0 & 1 & 0 & 0 & 0 & 0 & 0 & 0 & 0.875 & 0.125 & 0.375 & 0.625 \\
\hline$i 11$ & 0 & 0 & 0 & 0 & 0 & 0 & 0 & 0 & 0 & 0 & 1 & 0 & 0 & 0 & 0 & 0 & 0.875 & 0.125 & 0.375 & 0.625 \\
\hline$\lcm{112}$ & 0 & 0 & 0 & 0 & 0 & 0 & 0 & 0 & 0 & 0 & 0 & 1 & 0 & 0 & 0 & 0 & 0.875 & 0.375 & 0.125 & 0.625 \\
\hline i13 & 0 & 0 & 0 & 0 & 0 & 0 & 0 & 0 & 0 & 0 & 0 & 0 & 1 & 0 & 0 & 0 & 0.875 & 0.625 & 0.375 & 0.125 \\
\hline$i 14$ & 0 & 0 & 0 & 0 & 0 & 0 & 0 & 0 & 0 & 0 & 0 & 0 & 0 & 1 & 0 & 0 & 0.875 & 0.375 & 0.125 & 0.625 \\
\hline$i 15$ & 0 & 0 & 0 & 0 & 0 & 0 & 0 & 0 & 0 & 0 & 0 & 0 & 0 & 0 & 1 & 0 & 0.875 & 0.375 & 0.125 & 0.625 \\
\hline$i 16$ & 0 & 0 & 0 & 0 & 0 & 0 & 0 & 0 & 0 & 0 & 0 & 0 & 0 & 0 & 0 & 1 & 0.875 & 0.625 & 0.125 & 0.375 \\
\hline
\end{tabular}

Page B.29 
Pattern File for Node 11 of the Sample Network

Modified Training Set

\begin{tabular}{|c|c|c|c|c|c|c|c|c|c|c|c|c|c|c|c|c|c|c|c|c|c|c|}
\hline \multirow{2}{*}{$\begin{array}{c}\text { Destination } \\
\text { Node }\end{array}$} & \multicolumn{16}{|c|}{ Input Vector } & \multicolumn{6}{|c|}{ Output Vector } \\
\hline & i 1 & i2 & i3 & $i 4$ & i 5 & $i 6$ & i 7 & i8 & 19 & $i 10$ & $i 11$ & $i 12$ & 113 & $i 14$ & $i 15$ & $i 16$ & 01 & 02 & 03 & 04 & 05 & 06 \\
\hline 1 & 1 & 0 & 0 & 0 & 0 & 0 & 0 & 0 & 0 & 0 & 0 & 0 & 0 & 0 & 0 & 0 & 0.08333 & 0.25 & 0.41667 & 0.58333 & 0.75 & 0.91667 \\
\hline 2 & 0 & 1 & 0 & 0 & 0 & 0 & 0 & 0 & 0 & 0 & 0 & 0 & 0 & $\underline{0}$ & 0 & 0 & 0.08333 & 0.41667 & 0.25 & 0.91667 & 0.58333 & 0.75 \\
\hline 3 & 0 & 요 & 1. & 0 & 0 & 0 & 0 & 0 & 0 & 0 & 0 & 0 & 0 & 0 & 0 & 0 & 0.08333 & 0.41667 & 0.25 & 0.75 & 0.58333 & 0.91667 \\
\hline 4 & 0 & 0 & 욤 & 1 & 0 & 0 & 0 & 0 & 0 & 0 & 0 & 0 & 0 & 0 & 0 & 0 & 0.08333 & 0.25 & 0.58333 & 0.41667 & 0.91667 & 0.75 \\
\hline 5 & 0 & 0 & 0 & 0 & 1 & 0 & 0 & 0 & 0 & 0 & 0 & 0 & 0 & 0 & 0 & 0 & 0.08333 & 0.25 & 0.58333 & 0.41667 & 0.91667 & 0.75 \\
\hline 6 & 0 & 0 & 0 & 0 & 0 & 1 & 0 & 요 & 0 & 0 & 0 & 0 & 0 & 0 & 0 & 0 & 0.25 & 0.58333 & 0.08333 & 0.91667 & 0.41667 & 0.75 \\
\hline 7 & 0 & 0 & 0 & 이 & 0 & 0 & 1 & 요 & 0 & 0 & 0 & 0 & 0 & 0 & 0 & 0 & 0.08333 & 0.25 & 0.41667 & 0.58333 & 0.75 & 0.91667 \\
\hline 8 & 0 & 0 & 0 & 0 & 0 & 0 & 0 & 1 & 0 & 0 & 0 & 0 & 0 & 0 & 0 & 0 & 0.25 & 0.08333 & 0.75 & 0.41667 & 0.91667 & 0.58333 \\
\hline 9 & 0 & 0 & 이 & 0 & 0 & 0 & 0 & 0 & 1 & 0 & 0 & 0 & 0 & 0 & 0 & 0 & 0.41667 & 0.08333 & 0.91667 & 0.25 & 0.75 & 0.58333 \\
\hline 10 & 0 & 0 & 0 & 이 & 0 & 0 & 0 & 0 & 0 & 1 & 0 & 0 & 0 & 0 & 0 & 0 & 0.41667 & 0.91667 & 0.08333 & 0.75 & 0.25 & 0.58333 \\
\hline 11 & 0 & 0 & 온 & 이 & 이 & 요 & 0 & 이 & 요 & 0 & 1 & 0 & 0 & 0 & 0 & 0 & & & & & & \\
\hline 12 & 0 & 0 & 0 & 0 & 0 & 0 & 0 & 이 & 0 & 0 & 0 & 1 & 0 & 0 & 0 & 0 & 0.75 & 0.41667 & 0.91667 & 0.08333 & 0.58333 & 0.25 \\
\hline 13 & 0 & 0 & 0 & 0 & 0 & 0 & 0 & 0 & 0 & 0 & 0 & 0 & 1 & 0 & 0 & 0 & 0.75 & 0.41667 & 0.91667 & 0.08333 & 0.58333 & 0.25 \\
\hline 14 & 0 & 요 & 0 & 0 & 0 & 0 & 0 & 0 & 0 & 0 & 0 & 0 & 0 & 1 & 0 & 0 & 0.75 & 0.91667 & 0.25 & 0.58333 & 0.08333 & 0.41667 \\
\hline 15 & 0 & 0 & 0 & 아 & 아 & 0 & 0 & 0 & 인 & 0 & 0 & 0 & 0 & 0 & 1 & 0 & 0.91667 & 0.75 & 0.58333 & 0.25 & 0.41667 & 0.08333 \\
\hline 16 & 이 & 0 & 0 & 0 & 이 & 0 & 0 & 0 & 0 & 0 & 0 & 0 & 0 & 0 & 0 & 1 & 0.91667 & 0.75 & 0.58333 & 0.25 & 0.41667 & 0.08333 \\
\hline
\end{tabular}

Page B.30 
Training Results for V2.2.08.2(M)

(Network 2.08.2, V2 Macro, modified training set)

\begin{tabular}{|c|c|c|c|c|c|c|}
\hline \multirow[t]{2}{*}{ Destination } & \multicolumn{3}{|c|}{ Neuron o1 (Route '3) } & \multicolumn{3}{|c|}{ Neuron o2 (Route 6) } \\
\hline & Target & Meas & Diff & Target & Meas & Diff \\
\hline$i 1$ & 0.25 & 0.25082 & -0.0008 & 0.75 & 0.75101 & -0.001 \\
\hline$i 2$ & & 0.43514 & -0.4351 & & 0.43082 & -0.4308 \\
\hline i3 & 0.25 & 0.25266 & -0.0027 & 0.75 & 0.75178 & -0.0018 \\
\hline i4 & 0.25 & 0.25483 & -0.0048 & 0.75 & 0.75288 & -0.0029 \\
\hline i5 & 0.25 & 0.25081 & -0.0008 & 0.75 & 0.75098 & -0.001 \\
\hline i6 & 0.75 & 0.75031 & -0.0003 & 0.25 & 0.24928 & 0.00072 \\
\hline i 7 & 0.25 & 0.25088 & -0.0009 & 0.75 & 0.75093 & -0.0009 \\
\hline $\mathrm{i} 8$ & 0.25 & 0.24995 & $5 \mathrm{E}-05$ & 0.75 & 0.7507 & -0.0007 \\
\hline i9 & 0.25 & 0.25259 & -0.0026 & 0.75 & 0.75166 & -0.0017 \\
\hline$\$ 10$ & 0.75 & 0.74899 & 0.00101 & 0.25 & 0.24573 & 0.00427 \\
\hline$i 11$ & 0.25 & 0.24362 & 0.0 & 0.75 & 0.74795 & 0.00205 \\
\hline$i 12$ & 0.25 & 0.24463 & 0.0 & 0.75 & 0.74849 & 0.00151 \\
\hline$i 13$ & 0.25 & 0.25241 & -0.0024 & 0.75 & 0.7 & -0.0017 \\
\hline 114 & 0.75 & 0.74892 & 0.00108 & 0.25 & 0.24514 & 0.00486 \\
\hline$i 15$ & 0.75 & 0.75397 & -0.004 & 0.25 & 0.25943 & -0.0094 \\
\hline$i 16$ & 0.75 & 0.75125 & -0.0012 & 0.25 & 0.25197 & -0.002 \\
\hline \multirow{4}{*}{$\begin{array}{l}\text { Neuron } \\
\text { Results }\end{array}$} & \multicolumn{2}{|c|}{ Average |Diff| (Full) } & 0.02935 & \multicolumn{2}{|c|}{ Average |Diff| (Full) } & 0.02921 \\
\hline & \multicolumn{2}{|c|}{ Average |Diff| (Mod) } & 0.00229 & \multicolumn{2}{|c|}{ Average |Diff| (Mod) } & 0.00243 \\
\hline & \multirow{2}{*}{\multicolumn{2}{|c|}{ Std Dev $\mid$ Diff $\mid$ (Full) }} & 0.10823 & \multicolumn{2}{|c|}{ Std Dev |Diff| (Full) } & 0.10712 \\
\hline & & & 0.00199 & \multicolumn{2}{|c|}{ Std Dev |Diff $\mid$ (Mod) } & 0.00229 \\
\hline \multirow{2}{*}{$\begin{array}{l}\text { Network } \\
\text { Results }\end{array}$} & \multicolumn{2}{|c|}{ Average $\mid$ Diff $\mid$ (Full) } & 0.02928 & \multirow{2}{*}{\multicolumn{2}{|c|}{\begin{tabular}{|l|l|} 
Std Dev $\mid$ Diff & (Full) \\
Std Dev & Diff $\mid$ (Mod) \\
\end{tabular}}} & 01 \\
\hline & Average $\mid[$ & iffl (Mod) & 0.00236 & & & 0.00211 \\
\hline
\end{tabular}


Training Results for V2.2.08.4(M)

(Network 2.08.4, V2 Macro, modified training set)

\begin{tabular}{|c|c|c|c|c|c|c|}
\hline \multirow[t]{2}{*}{ Destination } & \multicolumn{3}{|c|}{ Neuron o1 (Route 5) } & \multicolumn{3}{|c|}{ Neuron o2 (Route 8) } \\
\hline & Target & Meas & Diff & Target & Meas & Diff \\
\hline i1 & 0.125 & 0.1092 & 0.0158 & 0.375 & 0.35656 & 0.01844 \\
\hline i2 & 0.375 & 0.38464 & -0.0096 & 0.125 & 0.16896 & -0.044 \\
\hline i3 & 0.375 & 0.37422 & 0.00078 & 0.125 & 0.13245 & -0.0075 \\
\hline ¡4 & 0.375 & 0.37872 & -0.0037 & 0.125 & 0.14116 & -0.0162 \\
\hline i 5 & 0.125 & 0.12419 & 0.00081 & .375 & 0.38077 & -0.0058 \\
\hline i6 & 0.625 & 0.63012 & -0.0051 & 0.125 & 0.08691 & 0.03809 \\
\hline$i 7$ & 0.625 & 0.6257 & -0.0007 & 0.125 & 0.08052 & 0.04448 \\
\hline i 8 & 0.625 & 0.64197 & -0.017 & 0.125 & 0.07961 & 0.04539 \\
\hline i9 & & 0.17998 & -0.18 & & 106 & -0.0511 \\
\hline$i 10$ & 0.875 & 0.807 & 0.068 & 0.125 & 0.16234 & -0.0373 \\
\hline$i 11$ & 0.875 & 0.83401 & 0.04099 & 0.125 & 0.14268 & -0.0177 \\
\hline$i 12$ & 0.875 & 0.90865 & -0.0337 & 0.375 & 0.37002 & 0.00498 \\
\hline$\lceil 13$ & 0.875 & 0.8 & 0.01398 & 0.625 & 0.63012 & -0.0051 \\
\hline$\lceil 14$ & 0.875 & 0.9 & -0.0345 & 0.375 & 0.36959 & 0.00541 \\
\hline 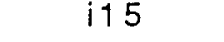 & 0.875 & 0.90949 & -0.0345 & 0.375 & 0.37099 & 0.00401 \\
\hline$i 16$ & 0.875 & 0.92616 & -0.0512 & 0.625 & 0.61295 & 0.01205 \\
\hline \multirow{4}{*}{$\begin{array}{l}\text { Neuron } \\
\text { Results }\end{array}$} & \multicolumn{2}{|c|}{ Average $\mid$ Diff $\mid$ (Full) } & 0.03189 & \multicolumn{2}{|c|}{ Average |Diff| (Full) } & 0.02234 \\
\hline & \multicolumn{2}{|c|}{ Average |Diff| (Mod) } & 0.02202 & \multicolumn{2}{|c|}{ Average |Diff] (Mod) } & 0.02042 \\
\hline & \multicolumn{2}{|c|}{\begin{tabular}{|l|l|} 
Std Dev $\mid$ Diff $\mid$ (Full) \\
\end{tabular}} & 0.04429 & \multicolumn{2}{|c|}{ Std Dev |Diff| (Full) } & 0.01767 \\
\hline & \multicolumn{2}{|c|}{ Std Dev $\mid$ Diff $\mid$ (Mod) } & 0.02075 & \multicolumn{2}{|c|}{ Std Dev |Diff (Mod) } & 0.01649 \\
\hline
\end{tabular}

\begin{tabular}{|c|c|c|c|c|c|c|}
\hline \multirow[t]{2}{*}{ Destination } & \multicolumn{3}{|c|}{ Neuron o3 (Route 12) } & \multicolumn{3}{|c|}{ Neuron o4 (Route 13) } \\
\hline & Target & Meas & Diff & Target & Meas & Diff \\
\hline i1 & 0.625 & 0.63668 & -0.0117 & 0.875 & 0.87536 & -0.0004 \\
\hline$i 2$ & 0.625 & 0.6008 & 0.0242 & 0.875 & 0.8902 & -0.0152 \\
\hline i3 & 0.625 & 0.61008 & 0.01492 & 0.875 & 0.90257 & -0.0276 \\
\hline i 4 & 0.625 & 0.60694 & 0.01806 & 0.875 & 0.90012 & -0.0251 \\
\hline i5 & 0.625 & 0.62944 & -0.0044 & 0.875 & 0.86247 & 0.01253 \\
\hline$i 6$ & 0.375 & 0.42094 & -0.0459 & 0.875 & 0.8203 & 0.0547 \\
\hline$i 7$ & 0.375 & 0.42361 & -0.0486 & 0.875 & 0.82086 & 0.05414 \\
\hline i 8 & 0.375 & 0.41848 & -0.0435 & 0.875 & 0.81252 & 0.06248 \\
\hline i9 & & 0.06041 & -0.0604 & & 0.12549 & -0.1255 \\
\hline i10 & 0.375 & 0.28083 & 0.09417 & 0.625 & 0.72225 & -0.0972 \\
\hline$i 11$ & 0.375 & 0.28011 & 0.09489 & 0.625 & 0.70899 & -0.084 \\
\hline$i 12$ & 0.125 & 0.15639 & -0.0314 & 0.625 & 0.60561 & 0.01939 \\
\hline$i 13$ & 0.375 & 0.38026 & -0.0053 & 0.125 & 0.11959 & 0.00541 \\
\hline$i 14$ & 0.125 & 0.15353 & -0.0285 & 0.625 & 0.60572 & 0.01928 \\
\hline$i 15$ & 0.125 & 0.16594 & -0.0409 & 0.625 & 0.60074 & 0.02426 \\
\hline$\$ 16$ & 0.125 & 0.11439 & 0.01061 & 0.375 & 0.36871 & 0.00629 \\
\hline \multirow{4}{*}{$\begin{array}{l}\text { Neuron } \\
\text { Results }\end{array}$} & \multicolumn{2}{|c|}{ Average |Diff $\mid$ (Full) } & 0.0361 & \multicolumn{2}{|c|}{ Average |Diff| (Full) } & 0.03959 \\
\hline & \multicolumn{2}{|c|}{ Average |Diff $\mid$ (Mod) } & 0.03447 & \multicolumn{2}{|c|}{ Average [Diff (Mod) } & 0.03386 \\
\hline & \multicolumn{2}{|c|}{ Std Dev $\mid$ Diff $\mid$ (Full) } & 0.02822 & \multicolumn{2}{|c|}{ Std Dev |Diff| (Full) } & 0.03669 \\
\hline & \multicolumn{2}{|c|}{ Std Dev [Diff] (Mod) } & 0.02843 & \multicolumn{2}{|c|}{ Std Dev |Diff $\mid$ (Mod) } & 0.02967 \\
\hline
\end{tabular}

\begin{tabular}{|l|l|r|r|r|r|}
\hline $\begin{array}{l}\text { Network } \\
\text { Results }\end{array}$ & Average $\mid$ Diff $\mid$ (Full) & 0.03248 & Std Dev |Diff| (Full) & 0.03307 \\
\cline { 2 - 6 } & Average $\mid$ Diff $\mid$ (Mod) & 0.0277 & Std Dev Diff & (Mod) & 0.0247 \\
\hline
\end{tabular}


Training Results for V2.2.08.6(M)

(Network 2.08.6, V2 Macro, modified training set)

\begin{tabular}{|c|c|c|c|c|c|c|c|c|c|}
\hline \multirow[t]{2}{*}{ Destination } & \multicolumn{3}{|c|}{ Neuron o1 (Route 7) } & \multicolumn{3}{|c|}{ Neuron o2 (Route 8) } & \multicolumn{3}{|c|}{ Neuron 03 (Route 10) } \\
\hline & Target & Meas & Diff & Target & Meas & Diff & Target & Meas & Diff \\
\hline$i 1$ & 0.08333 & 0.06172 & 0.02161 & 0.25 & 0.2585 & -0.0085 & 0.41667 & 0.41389 & 0.00278 \\
\hline 12 & 0.08333 & 0.13664 & -0.0533 & 0.41667 & 0.43062 & -0.014 & 0.25 & 0.23642 & 0.01358 \\
\hline i3 & 0.08333 & 0.10874 & -0.0254 & 0.41667 & 0.398 & 0.01867 & 0.25 & 0.24862 & 0.00138 \\
\hline$i 4$ & 0.08333 & 0.09668 & -0.0133 & 0.25 & .20363 & 0.04637 & 0.58333 & 0.59691 & -0.0136 \\
\hline$i 5$ & 0.08333 & 0.09233 & -0.009 & 0.25 & 0.213 & 0.037 & 0.58333 & 0.59671 & -0.0134 \\
\hline i6 & 0.25 & 0.17169 & 0.07831 & 0.58333 & 0.61812 & -0.0348 & 0.08333 & 0.10494 & -0.0216 \\
\hline$i 7$ & 0.08333 & 0.05721 & 0.02612 & 0.25 & 0.27988 & -0.0299 & 0.41667 & 0.39973 & 0.01694 \\
\hline i 8 & 0.25 & 0.23902 & 0.01098 & 0.08333 & 0.13341 & -0.0501 & 0.75 & 0.82527 & -0.0753 \\
\hline i 9 & 0.41667 & 0.40024 & 0.01643 & 0.08333 & 0.14153 & -0.0582 & 0.91667 & 0.88947 & 0.0272 \\
\hline $\mathrm{i10}$ & 0.41667 & 0.45291 & -0.0362 & 0.91667 & 0.84433 & 0.07234 & 0.08333 & 0.10821 & -0.0249 \\
\hline$i 11$ & & 0.02135 & -0.0214 & & 0.03729 & -0.0373 & & 0.02667 & -0.0267 \\
\hline$i 12$ & 0.75 & 0.78417 & -0.0342 & 0.41667 & 0.3919 & 0.02477 & 0.91667 & 0.85977 & 0.0569 \\
\hline i13 & 0.75 & 0.78289 & -0.0329 & 0.41667 & 0.39392 & 0.02275 & 0.91667 & 0.85939 & 0.05728 \\
\hline$\lceil 14$ & 0.75 & 0.74397 & 0.00603 & 0.91667 & 0.8983 & 0.01837 & 0.25 & 0.20792 & 0.04208 \\
\hline$i 15$ & 0.91667 & 0.88108 & 0.03559 & 0.75 & 0.78922 & -0.0392 & 0.58333 & 0.61683 & -0.0335 \\
\hline$i 16$ & 0.91667 & 0.88152 & 0.03515 & 0.75 & 0.79066 & -0.0407 & 0.58333 & 0.61603 & -0.0327 \\
\hline \multirow{4}{*}{$\begin{array}{l}\text { Neuron } \\
\text { Results }\end{array}$} & \multicolumn{2}{|c|}{\begin{tabular}{l|l|l|l|} 
Average & Diff & (Full) \\
\end{tabular}} & 0.0285 & \multicolumn{2}{|c|}{ Average |Diff| (Full) } & 0.03455 & \multicolumn{2}{|c|}{ Average |Diff| (Full) } & 0.02873 \\
\hline & \multicolumn{2}{|c|}{ Average $\mid$ Diff $\mid$ (Mod) } & 0.02897 & \multicolumn{2}{|c|}{ Average |Diff| (Mod) } & 0.03437 & \multicolumn{2}{|c|}{ Average [Diff| (Mod) } & 0.02887 \\
\hline & \multicolumn{2}{|c|}{ Std Dev $\mid$ Diff $\mid$ (Full) } & 0.01823 & \multicolumn{2}{|c|}{ Std Dev |Diff $\mid$ (Full) } & 0.01696 & \multicolumn{2}{|c|}{ Std Dev |Diff| (Full) } & 0.02052 \\
\hline & \multicolumn{2}{|c|}{ Std Dev |Diff| (Mod) } & 0.01876 & \multicolumn{2}{|c|}{ Std Dev |Diff] (Mod) } & 0.01754 & \multicolumn{2}{|c|}{ Std Dev |Diff $\mid$ (Mod) } & 0.02124 \\
\hline
\end{tabular}

\begin{tabular}{|c|c|c|c|c|c|c|c|c|c|}
\hline \multirow[t]{2}{*}{ Destination } & \multicolumn{3}{|c|}{ Neuron o4 (Route 12) } & \multicolumn{3}{|c|}{ Neuron o5 (Route 14) } & \multicolumn{3}{|c|}{ Neuron o6 (Route 15) } \\
\hline & Target & Meas & Diff & Target & Meas & Diff & Target & Meas & Diff \\
\hline i 1 & 0.58333 & 0.58206 & 0.00127 & 0.75 & 0.81844 & -0.0684 & 0.91667 & 0.85316 & 0.06351 \\
\hline$i 2$ & 0.91667 & 0.84222 & 0.07445 & 0.58333 & 0.58151 & 0.00182 & 0.75 & 0.81554 & -0.0655 \\
\hline i3 & 0.75 & 0.80137 & -0.0514 & 0.58333 & 0.627 & -0.0437 & 0.91667 & 0.82676 & 0.08991 \\
\hline ¡ 4 & 0.41667 & 0.45756 & -0.0409 & 0.91667 & 0.83459 & 0.08208 & 0.75 & 0.80056 & -0.0506 \\
\hline ¡ 5 & 0.41667 & 0.43474 & -0.0181 & 0.91667 & 0.85022 & 0.06645 & 0.75 & 0.80398 & -0.054 \\
\hline i 6 & 0.91667 & 0.90707 & 0.0096 & 0.41667 & 0.36331 & 0.05336 & 0.75 & 0.7905 & -0.0405 \\
\hline i 7 & 0.58333 & 0.56772 & 0.01561 & 0.75 & 0.82371 & -0.0737 & 0.91667 & 0.86394 & 0.05273 \\
\hline i 8 & 0.41667 & 0.34146 & 0.07521 & 0.91667 & 0.82579 & 0.09088 & 0.58333 & 0.65121 & -0.0679 \\
\hline ¡9 & 0.25 & 0.27979 & -0.0298 & 0.75 & 0.81702 & -0.067 & 0.58333 & 0.54126 & 0.04207 \\
\hline$i 10$ & 0.75 & 0.79098 & -0.041 & 0.25 & 0.2244 & 0.0256 & 0.58333 & 0.58303 & 0.0003 \\
\hline$i 11$ & & 0.04389 & -0.0439 & & 0.04197 & -0.042 & & 0.0521 & -0.0521 \\
\hline$\lceil 12$ & 333 & 0.14505 & -0.0617 & 0.58333 & 0.62217 & -0.0388 & 0.25 & 0.20663 & 0.04337 \\
\hline$i 13$ & 0.08333 & 0.14216 & -0.0588 & 0.58333 & 0.62432 & -0.041 & 0.25 & 0.2061 & 0.0439 \\
\hline$i 14$ & 0.58333 & 0.59922 & -0.0159 & 0.08333 & 0.2221 & -0.1388 & 0.41667 & 0.3741 & 0.04257 \\
\hline$i 15$ & 0.25 & 0.20697 & 0.04303 & 0.41667 & 0.36066 & 0.05601 & 0.08333 & 0.15845 & -0.0751 \\
\hline$i 16$ & 0.25 & 0.20322 & 0.04678 & 0.41667 & 0.35996 & 0.05671 & 0.08333 & 0.15646 & -0.0731 \\
\hline \multirow{4}{*}{$\begin{array}{l}\text { Neuron } \\
\text { Results }\end{array}$} & \multicolumn{2}{|c|}{ Average |Diff (Full) } & 0.03921 & \multicolumn{2}{|c|}{ Average |Diff $\mid$ (Full) } & 0.05914 & \multicolumn{2}{|c|}{\begin{tabular}{|l|l|} 
Average & Diff \\
\end{tabular}} & 0.05357 \\
\hline & \multicolumn{2}{|c|}{\begin{tabular}{|l|l|} 
Average $\mid$ Diff & (Mod) \\
\end{tabular}} & 0.0389 & \multicolumn{2}{|c|}{\begin{tabular}{|l|l|} 
Average $\mid$ Diff & (Mod) \\
\end{tabular}} & 0.06029 & \multicolumn{2}{|c|}{ Average $\mid$ Diff $\mid$ (Mod) } & 0.05367 \\
\hline & \multirow{2}{*}{\multicolumn{2}{|c|}{\begin{tabular}{|l|l|l|} 
Std Dev $\mid$ Diff & (Full) \\
Std & (Diff \\
\end{tabular}}} & 0.02255 & \multicolumn{2}{|c|}{ Std Dev |Diff| (Full) } & 0.03058 & \multirow{2}{*}{\multicolumn{2}{|c|}{\begin{tabular}{|l|l|} 
Std Dev & Diff \\
Std Dev IDiff & (Mod) \\
\end{tabular}}} & 0.02014 \\
\hline & & & 0.02331 & Std Dev ID & iff (Mod) & 0.03129 & & & 0.02084 \\
\hline
\end{tabular}

\begin{tabular}{|l|l|l|l|l|l|}
\hline $\begin{array}{l}\text { Network } \\
\text { Results }\end{array}$ & Average $\mid$ Diff| (Full) & 0.04062 & Std Dev $\mid$ Diff $\mid$ (Full) & 0.02444 \\
\cline { 2 - 6 } & Average Diff $\mid$ (Mod) & 0.04084 & Std Dev $\mid$ Diff $\mid$ (Mod) & 0.02509 \\
\hline
\end{tabular} 
Appendix C:

Full network modelling results 
Routing Results for Node 1

of the Sample Network

\begin{tabular}{|c|c|c|c|c|}
\hline \multirow{2}{*}{$\begin{array}{c}\text { Destination } \\
\text { Node }\end{array}$} & \multicolumn{4}{|c|}{ Real Output Values } \\
\hline & 01 & 02 & 03 & 04 \\
\hline 11 & & & & \\
\hline 12 & 0.25685 & 0.49057 & 0.60218 & 1 \\
\hline$i 3$ & 0.1573 & 0.39102 & 0.50263 & 1 \\
\hline$i 4$ & 0.38132 & 0.167 & 0.27861 & 1 \\
\hline$i 5$ & 0.47713 & 0.26281 & 0.1828 & 1 \\
\hline 16 & 0.26626 & 0.40625 & 0.51786 & 1 \\
\hline 17 & 0.28177 & 0.26655 & 0.37816 & 1 \\
\hline 18 & 0.39153 & 0.28067 & 0.39228 & 0.9822 \\
\hline 19 & 0.49463 & 0.38377 & 0.3708 & 0.9472 \\
\hline$i 10$ & 0.38513 & 0.47941 & 0.59102 & 1 \\
\hline$i 11$ & 0.39153 & 0.37631 & 0.48792 & 0.9159 \\
\hline 112 & 0.5104 & 0.45707 & 0.5122 & 0.8058 \\
\hline 113 & 0.6772 & 0.59647 & 0.5835 & 0.7553 \\
\hline 114 & 0.47326 & 0.52121 & 0.63282 & 0.88936 \\
\hline 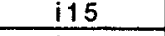 & 0.52703 & 0.51181 & 0.62196 & 0.7804 \\
\hline$i 16$ & 0.70733 & 0.66277 & 0.7387 & 0.6001 \\
\hline
\end{tabular}

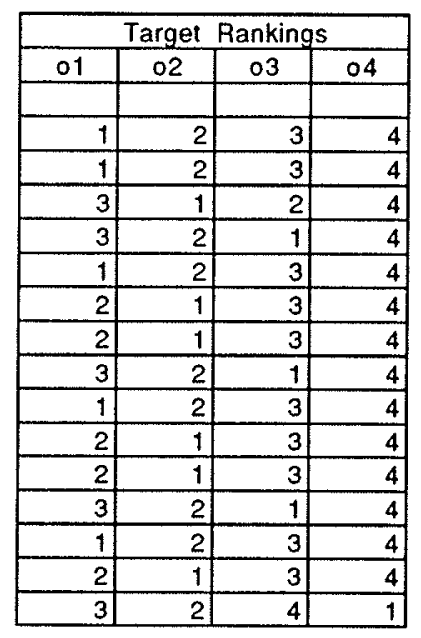

\begin{tabular}{|l|l|l|l|}
\hline \multicolumn{4}{|c|}{ Target Values } \\
\hline 01 & 02 & 03 & 04 \\
\hline & & & \\
\hline 0.125 & 0.375 & 0.625 & 0.875 \\
\hline 0.125 & 0.375 & 0.625 & 0.875 \\
\hline 0.625 & 0.125 & 0.375 & 0.875 \\
\hline 0.625 & 0.375 & 0.125 & 0.875 \\
\hline 0.125 & 0.375 & 0.625 & 0.875 \\
\hline 0.375 & 0.125 & 0.625 & 0.875 \\
\hline 0.375 & 0.125 & 0.625 & 0.875 \\
\hline 0.625 & 0.375 & 0.125 & 0.875 \\
\hline 0.125 & 0.375 & 0.625 & 0.875 \\
\hline 0.375 & 0.125 & 0.625 & 0.875 \\
\hline 0.375 & 0.125 & 0.625 & 0.875 \\
\hline 0.625 & 0.375 & 0.125 & 0.875 \\
\hline 0.125 & 0.375 & 0.625 & 0.875 \\
\hline 0.375 & 0.125 & 0.625 & 0.875 \\
\hline 0.625 & 0.375 & 0.875 & 0.125 \\
\hline
\end{tabular}

\begin{tabular}{|c|}
\hline $\begin{array}{c}\text { Destination } \\
\text { Node }\end{array}$ \\
\hline$i 1$ \\
\hline 12 \\
\hline 13 \\
\hline 14 \\
\hline$i 5$ \\
\hline$i 6$ \\
\hline 17 \\
\hline 18 \\
\hline 19 \\
\hline$i 10$ \\
\hline 111 \\
\hline$i 12$ \\
\hline$i 13$ \\
\hline$i 14$ \\
\hline 115 \\
\hline 116 \\
\hline
\end{tabular}
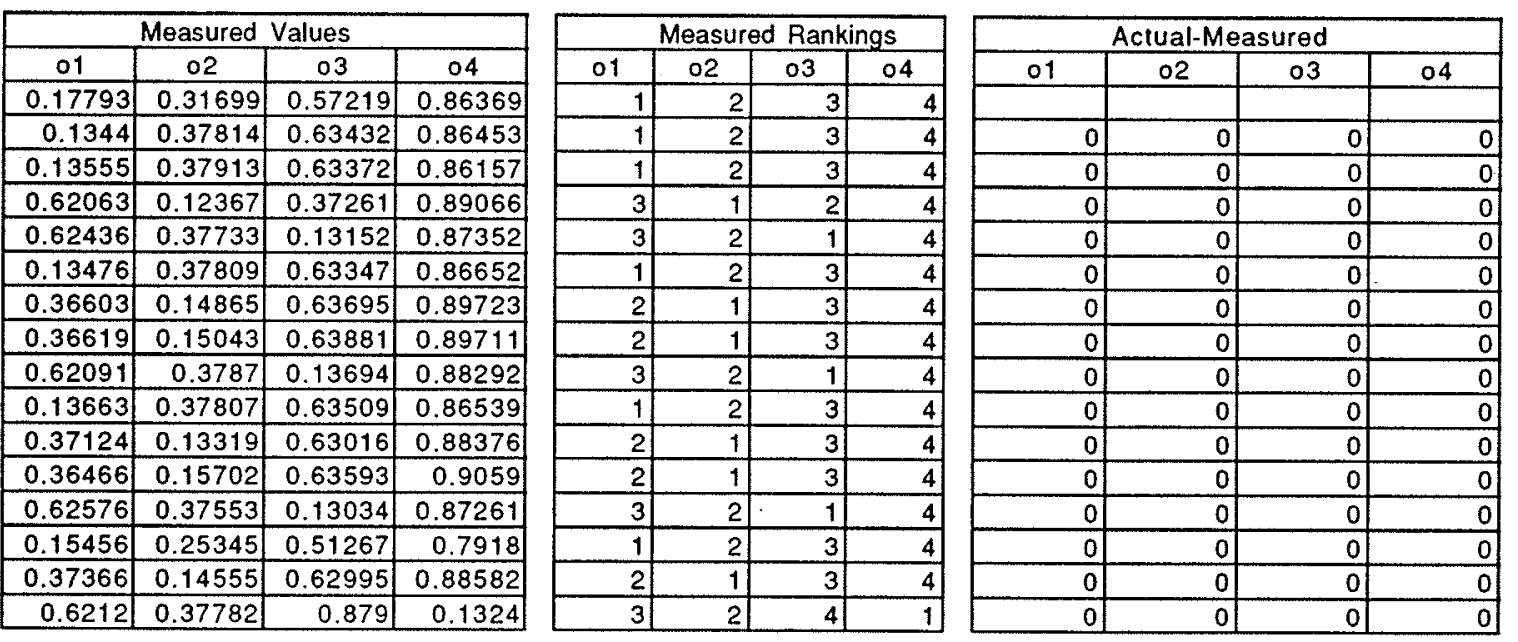

Page C.1 
Routing Results for Node 2

of the Sample Network

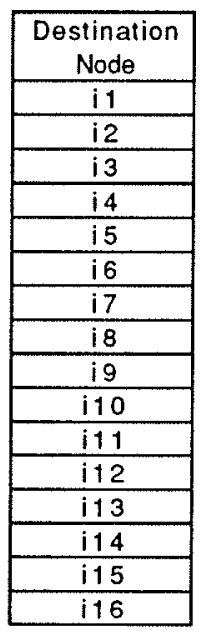

\begin{tabular}{|c|c|}
\hline \multicolumn{2}{|c|}{ Real Output Values } \\
\hline 01 & 02 \\
\hline 0.2569 & 0.3986 \\
\hline & \\
\hline 0.1245 & 0.2663 \\
\hline 0.3486 & 0.3966 \\
\hline 0.4146 & 0.4924 \\
\hline 0.2335 & 0.1573 \\
\hline 0.249 & 0.297 \\
\hline 0.3588 & 0.4068 \\
\hline 0.4618 & 0.5097 \\
\hline 0.3524 & 0.2762 \\
\hline 0.3588 & 0.3793 \\
\hline 0.4776 & 0.4981 \\
\hline 0.6385 & 0.659 \\
\hline 0.4405 & 0.3643 \\
\hline 0.4942 & 0.4733 \\
\hline 0.6745 & 0.6536 \\
\hline
\end{tabular}

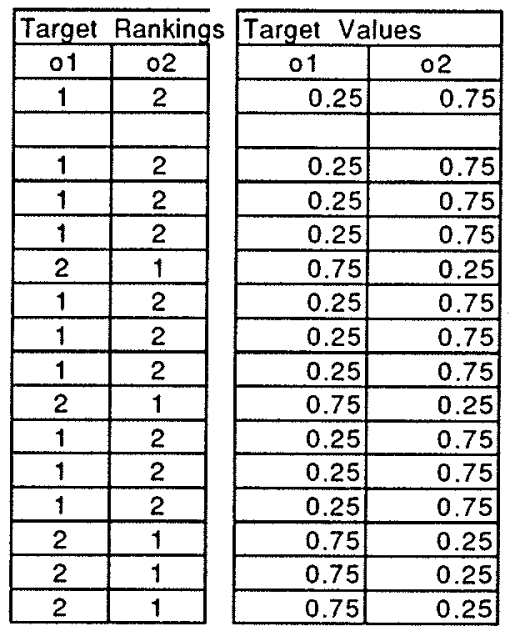

\begin{tabular}{|c|}
\hline $\begin{array}{c}\text { Destination } \\
\text { Node }\end{array}$ \\
\hline$i 1$ \\
\hline$i 2$ \\
\hline$i 3$ \\
\hline$i 4$ \\
\hline$i 5$ \\
\hline$i 6$ \\
\hline$i 7$ \\
\hline$i 8$ \\
\hline$i 9$ \\
\hline$i 10$ \\
\hline$i 11$ \\
\hline$i 12$ \\
\hline$i 13$ \\
\hline$i 14$ \\
\hline$i 15$ \\
\hline$i 16$ \\
\hline
\end{tabular}

\begin{tabular}{|c|c|}
\hline \multicolumn{2}{|c|}{ Measured Values } \\
\hline 01 & 02 \\
\hline 0.25082 & 0.75101 \\
\hline 0.43514 & 0.43082 \\
\hline 0.25266 & 0.75178 \\
\hline 0.25483 & 0.75288 \\
\hline 0.25081 & 0.75098 \\
\hline 0.75031 & 0.24928 \\
\hline 0.25088 & 0.75093 \\
\hline 0.24995 & 0.7507 \\
\hline 0.25259 & 0.75166 \\
\hline 0.74899 & 0.24573 \\
\hline 0.24362 & 0.74795 \\
\hline 0.24463 & 0.74849 \\
\hline 0.25241 & 0.75174 \\
\hline 0.74892 & 0.24514 \\
\hline 0.75397 & 0.25943 \\
\hline 0.75125 & 0.25197 \\
\hline
\end{tabular}
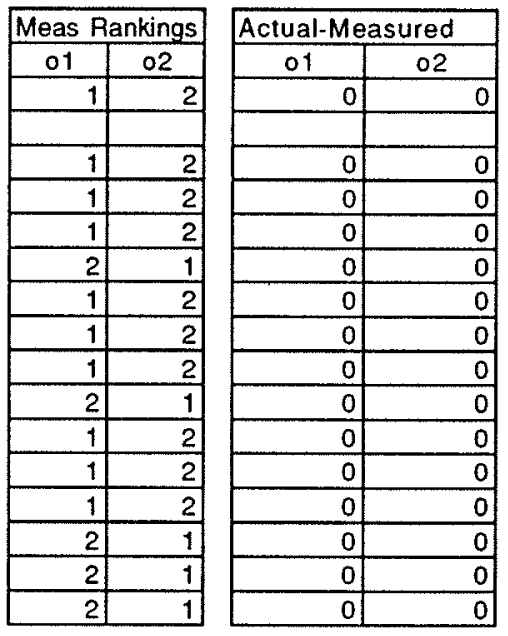

Page C.2 
Routing Results for Node 3

of the Sample Network

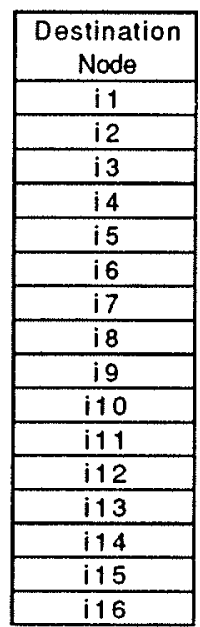

\begin{tabular}{|c|c|c|c|}
\hline \multicolumn{5}{|c|}{ Real Output Values } \\
\hline 01 & 02 & 03 & 04 \\
\hline 0.1573 & 0.6381 & 0.5152 & 0.39102 \\
\hline 0.67085 & 0.12455 & 0.26626 & 0.42146 \\
\hline & & & \\
\hline 0.2993 & 0.4961 & 0.37321 & 0.24902 \\
\hline 0.3151 & 0.59191 & 0.46902 & 0.34483 \\
\hline 0.53855 & 0.25685 & 0.13396 & 0.28917 \\
\hline 0.39885 & 0.39655 & 0.27366 & 0.14947 \\
\hline 0.41297 & 0.50631 & 0.38342 & 0.25923 \\
\hline 0.5031 & 0.60941 & 0.48652 & 0.36233 \\
\hline 0.61171 & 0.37572 & 0.25283 & 0.36233 \\
\hline 0.50861 & 0.47882 & 0.35593 & 0.25923 \\
\hline 0.58937 & 0.59769 & 0.4748 & 0.3781 \\
\hline 0.7158 & 0.75849 & 0.6356 & 0.5389 \\
\hline 0.65351 & 0.46385 & 0.34096 & 0.40413 \\
\hline 0.64411 & 0.57281 & 0.44992 & 0.39473 \\
\hline 0.7324 & 0.75311 & 0.63022 & 0.57503 \\
\hline
\end{tabular}

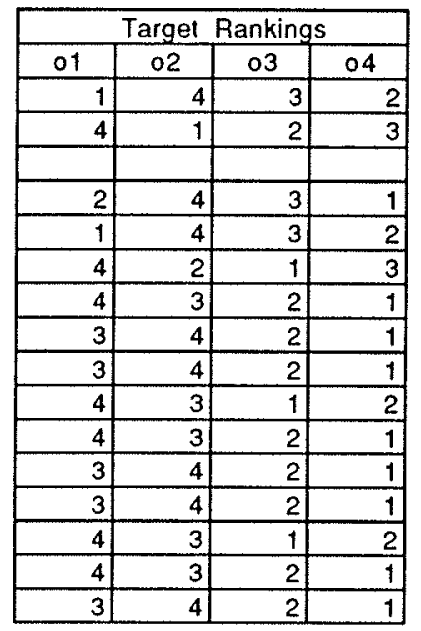

\begin{tabular}{|l|l|l|l|}
\hline \multicolumn{4}{|c|}{ Target Values } \\
\hline 01 & 02 & 03 & 04 \\
\hline 0.125 & 0.875 & 0.625 & 0.375 \\
\hline 0.875 & 0.125 & 0.375 & 0.625 \\
\hline & & & \\
\hline 0.375 & 0.875 & 0.625 & 0.125 \\
\hline 0.125 & 0.875 & 0.625 & 0.375 \\
\hline 0.875 & 0.375 & 0.125 & 0.625 \\
\hline 0.875 & 0.625 & 0.375 & 0.125 \\
\hline 0.625 & 0.875 & 0.375 & 0.125 \\
\hline 0.625 & 0.875 & 0.375 & 0.125 \\
\hline 0.875 & 0.625 & 0.125 & 0.375 \\
\hline 0.875 & 0.625 & 0.375 & 0.125 \\
\hline 0.625 & 0.875 & 0.375 & 0.125 \\
\hline 0.625 & 0.875 & 0.375 & 0.125 \\
\hline 0.875 & 0.625 & 0.125 & 0.375 \\
\hline 0.875 & 0.625 & 0.375 & 0.125 \\
\hline 0.625 & 0.875 & 0.375 & 0.125 \\
\hline
\end{tabular}

\begin{tabular}{|c|}
\hline $\begin{array}{c}\text { Destination } \\
\text { Node }\end{array}$ \\
\hline$i 1$ \\
\hline$i 2$ \\
\hline$i 3$ \\
\hline$i 4$ \\
\hline$i 5$ \\
\hline$i 6$ \\
\hline$i 7$ \\
\hline$i 8$ \\
\hline$i 9$ \\
\hline$i 10$ \\
\hline$i 11$ \\
\hline$i 12$ \\
\hline$i 13$ \\
\hline$i 14$ \\
\hline$i 15$ \\
\hline$i 16$ \\
\hline
\end{tabular}

\begin{tabular}{|c|r|r|r|}
\hline \multicolumn{5}{|c|}{ Measured Values } \\
\hline 01 & \multicolumn{1}{|c|}{02} & \multicolumn{1}{|c|}{03} & \multicolumn{1}{c|}{04} \\
\hline 0.12151 & 0.8926 & 0.61879 & 0.37285 \\
\hline 0.86982 & 0.12291 & 0.37361 & 0.62831 \\
\hline 0.01196 & 0.00902 & 0.00738 & 0.00665 \\
\hline 0.38071 & 0.90741 & 0.58221 & 0.1769 \\
\hline 0.12104 & 0.89017 & 0.61558 & 0.37972 \\
\hline 0.88732 & 0.36894 & 0.14916 & 0.61436 \\
\hline 0.85143 & 0.6956 & 0.27 & 0.15719 \\
\hline 0.62595 & 0.84305 & 0.4083 & 0.09028 \\
\hline 0.62685 & 0.83708 & 0.40614 & 0.09996 \\
\hline 0.89895 & 0.58566 & 0.18704 & 0.37965 \\
\hline 0.8604 & 0.67518 & 0.31801 & 0.13231 \\
\hline 0.62621 & 0.83959 & 0.40877 & 0.079 \\
\hline 0.62563 & 0.84102 & 0.40882 & 0.08469 \\
\hline 0.89937 & 0.59368 & 0.18942 & 0.36824 \\
\hline 0.85911 & 0.67721 & 0.30818 & 0.14161 \\
\hline 0.62597 & 0.8392 & 0.40903 & 0.88541 \\
\hline
\end{tabular}
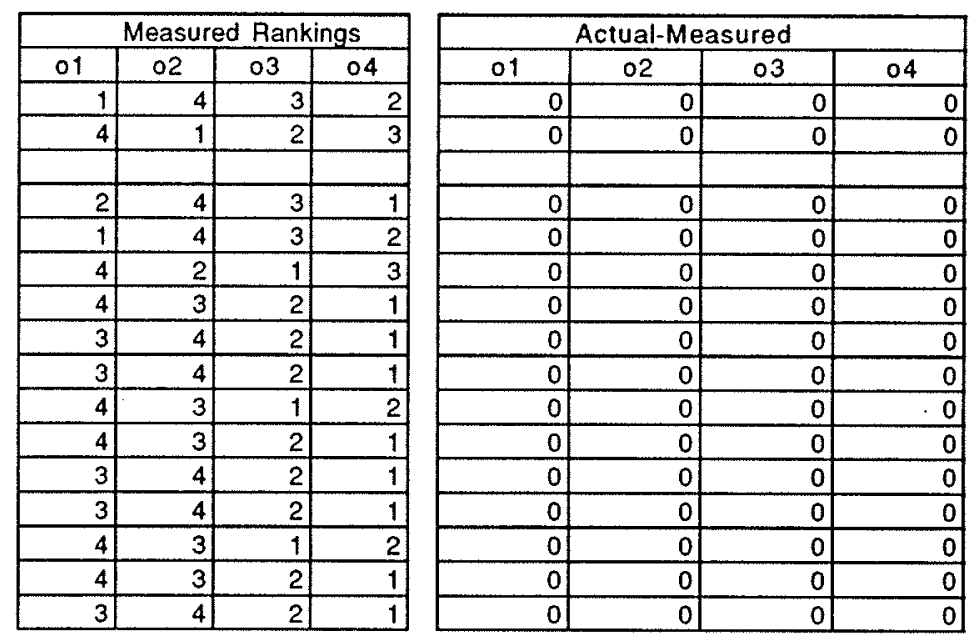

Page C.3 
Routing Results for Node 4

of the Sample Network

\begin{tabular}{|c|c|c|c|}
\hline $\begin{array}{c}\text { Destination } \\
\text { Node }\end{array}$ \\
\hline$i 1$ \\
\hline$i 2$ \\
\hline 13 \\
\hline$i 4$ \\
\hline 15 \\
\hline 16 \\
\hline 0.167 & 0.27861 & 0.38132 & 0.5052 \\
\hline 0.39885 & 0.51046 & 0.34857 & 0.47245 \\
\hline 0.2993 & 0.41091 & 0.24902 & 0.3729 \\
\hline & & & \\
\hline 0.3248 & 0.12081 & 0.52541 & 0.42976 \\
\hline 18 \\
\hline 19 \\
\hline 110 \\
\hline$i 11$ \\
\hline$i 12$ \\
\hline 113 \\
\hline 0.40826 & 0.51987 & 0.26425 & 0.27343 \\
\hline 0.42376 & 0.52167 & 0.12455 & 0.24843 \\
\hline 0.53353 & 0.41191 & 0.23431 & 0.13867 \\
\hline 0.5128 & 0.30881 & 0.33741 & 0.24177 \\
\hline 0.52713 & 0.63874 & 0.33741 & 0.38667 \\
\hline 0.53353 & 0.55681 & 0.23431 & 0.28357 \\
\hline 0.6524 & 0.45021 & 0.35318 & 0.31507 \\
\hline 0.7255 & 0.52151 & 0.51398 & 0.45447 \\
\hline 0.61526 & 0.66893 & 0.37921 & 0.42847 \\
\hline 115 \\
\hline 0.66903 & 0.55997 & 0.36981 & 0.41907 \\
\hline 0.7421 & 0.65591 & 0.55011 & 0.52077 \\
\hline
\end{tabular}

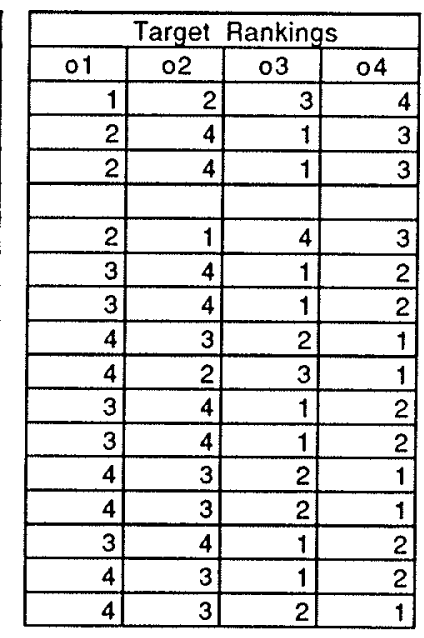

\begin{tabular}{|l|l|l|l|}
\hline \multicolumn{4}{|c|}{ Target Values } \\
\hline 01 & 02 & 03 & 04 \\
\hline 0.125 & 0.375 & 0.625 & 0.875 \\
\hline 0.375 & 0.875 & 0.125 & 0.625 \\
\hline 0.375 & 0.875 & 0.125 & 0.625 \\
\hline & & & \\
\hline 0.375 & 0.125 & 0.875 & 0.625 \\
\hline 0.625 & 0.875 & 0.125 & 0.375 \\
\hline 0.625 & 0.875 & 0.125 & 0.375 \\
\hline 0.875 & 0.625 & 0.375 & 0.125 \\
\hline 0.875 & 0.375 & 0.625 & 0.125 \\
\hline 0.625 & 0.875 & 0.125 & 0.375 \\
\hline 0.625 & 0.875 & 0.125 & 0.375 \\
\hline 0.875 & 0.625 & 0.375 & 0.125 \\
\hline 0.875 & 0.625 & 0.375 & 0.125 \\
\hline 0.625 & 0.875 & 0.125 & 0.375 \\
\hline 0.875 & 0.625 & 0.125 & 0.375 \\
\hline 0.875 & 0.625 & 0.375 & 0.125 \\
\hline
\end{tabular}

\begin{tabular}{|c|}
\hline $\begin{array}{c}\text { Destination } \\
\text { Node }\end{array}$ \\
\hline$i 1$ \\
\hline$i 2$ \\
\hline$i 3$ \\
\hline$i 4$ \\
\hline$i 5$ \\
\hline$i 6$ \\
\hline$i 7$ \\
\hline$i 8$ \\
\hline$i 9$ \\
\hline$i 10$ \\
\hline$i 11$ \\
\hline$i 12$ \\
\hline$i 13$ \\
\hline$i 14$ \\
\hline$i 15$ \\
\hline$i 16$ \\
\hline
\end{tabular}
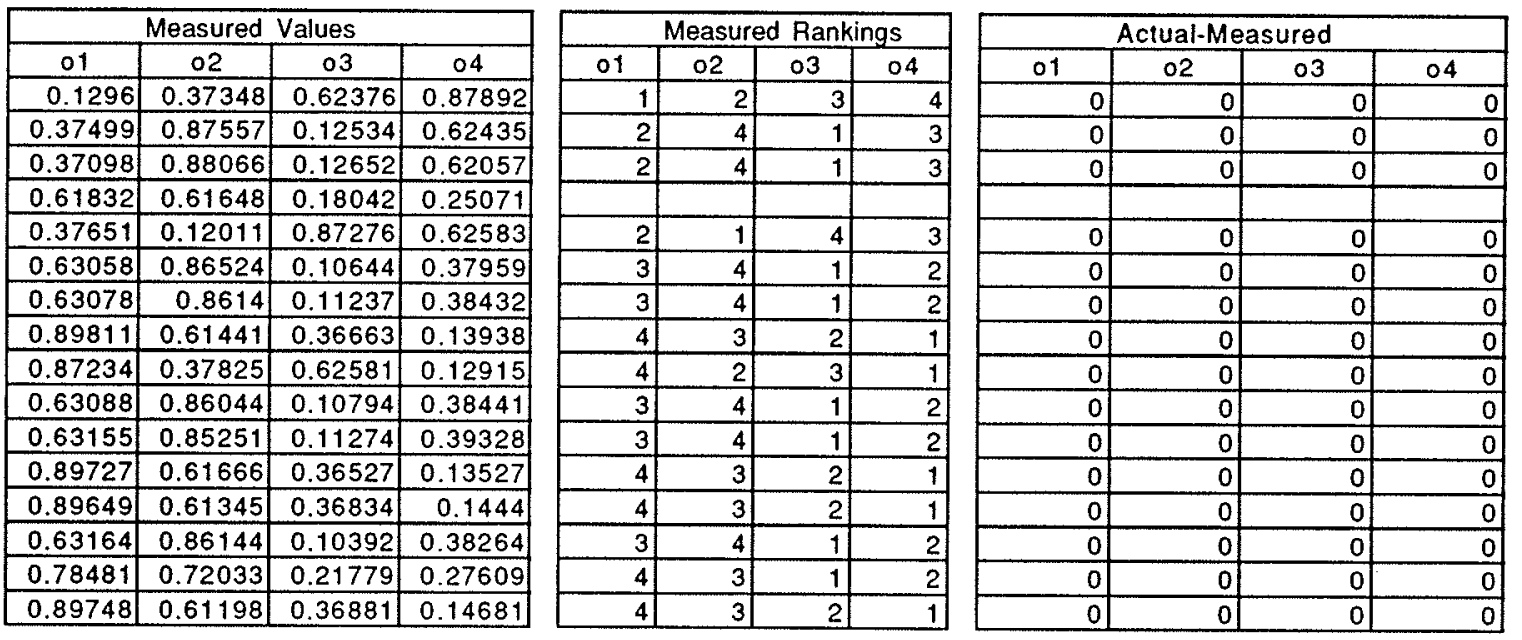

Page C.4 
Routing Results for Node 5

of the Sample Network

\begin{tabular}{|c|}
\hline $\begin{array}{c}\text { Destination } \\
\text { Node }\end{array}$ \\
\hline$i 1$ \\
\hline 12 \\
\hline 13 \\
\hline$i 4$ \\
\hline 15 \\
\hline 16 \\
\hline 17 \\
\hline 18 \\
\hline$i 9$ \\
\hline 110 \\
\hline$i 11$ \\
\hline$i 12$ \\
\hline$i 13$ \\
\hline 114 \\
\hline 115 \\
\hline 116 \\
\hline
\end{tabular}

\begin{tabular}{|r|c|r|}
\hline \multicolumn{3}{|c|}{ Real Output Values } \\
\hline 01 & \multicolumn{1}{|c|}{02} & \multicolumn{1}{c|}{03} \\
\hline 0.1828 & 0.26281 & 0.57177 \\
\hline 0.41465 & 0.44438 & 0.64988 \\
\hline 0.3151 & 0.34483 & 0.55032 \\
\hline 0.3248 & 0.12081 & 0.42977 \\
\hline & & \\
\hline 0.42406 & 0.36006 & 0.56556 \\
\hline 0.42435 & 0.22036 & 0.34776 \\
\hline 0.43847 & 0.23448 & 0.3161 \\
\hline 0.54157 & 0.33758 & 0.213 \\
\hline 0.54293 & 0.43322 & 0.5641 \\
\hline 0.53411 & 0.33012 & 0.461 \\
\hline 0.61486 & 0.41088 & 0.3544 \\
\hline 0.75427 & 0.55028 & 0.4257 \\
\hline 0.63106 & 0.47502 & 0.57312 \\
\hline 0.66961 & 0.46562 & 0.46416 \\
\hline 0.7579 & 0.61658 & 0.5601 \\
\hline
\end{tabular}

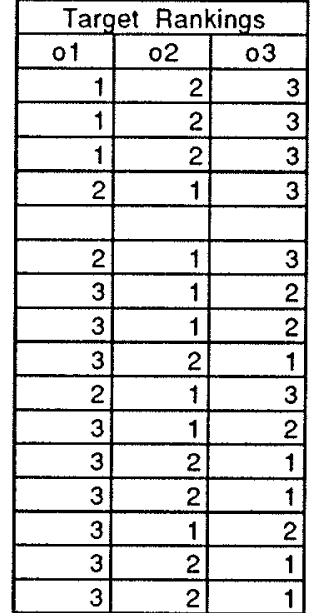

\begin{tabular}{|c|r|c|}
\hline \multicolumn{3}{|c|}{ Target Values } \\
\hline 01 & 02 & 03 \\
\hline 0.16667 & 0.5 & 0.83333 \\
\hline 0.16667 & 0.5 & 0.83333 \\
\hline 0.16667 & 0.5 & 0.83333 \\
\hline
\end{tabular}

\begin{tabular}{r|r|r|}
\hline 0.16667 & 0.16667 & 0.83333 \\
\hline
\end{tabular}

0.5

\begin{tabular}{l|l|l|} 
& & \\
\hline 0.5 & 0.16667 & 0.83333 \\
\hline
\end{tabular}

\begin{tabular}{rrr}
0.83333 & 0.16667 & 0.5 \\
\hline
\end{tabular}

\begin{tabular}{|l|l|l|}
\hline 0.833333 & 0.16667 & 0.5 \\
\hline 0.8333 &
\end{tabular}

\begin{tabular}{r|r|r}
\hline 0.83333 & 0.5 & 0.16667 \\
\hline 0.5 & 0.16667 & 0.83333 \\
\hline
\end{tabular}

\begin{tabular}{l|l|l|}
0.5 & 0.16667 & 0.83333 \\
\hline
\end{tabular}

\begin{tabular}{r|r}
0.83333 & 0.16667 \\
\hline 0.83333 & 0.5 \\
\hline
\end{tabular}

\begin{tabular}{ll|l}
0.83333 & 0.16667 \\
\hline 0.83333 & 0.5 & 0. \\
\hline
\end{tabular}

16667

\begin{tabular}{r|r|r|}
\hline 0.83333 & 0.5 & 0.16667 \\
\hline 0.83333 & 0.16667 & 0.5 \\
\hline 0.83333 & 0.5 & 0.16667 \\
\hline
\end{tabular}

\begin{tabular}{|r|r|}
\hline 0.83333 & 0.16667 \\
\hline 0.83333 & 0.5 \\
\hline
\end{tabular}

\begin{tabular}{l|l|l|}
\hline 0.7579 & 0.61658 & 0.5601 \\
\hline
\end{tabular}

\begin{tabular}{|c|}
\hline $\begin{array}{c}\text { Destination } \\
\text { Node }\end{array}$ \\
\hline$i 1$ \\
\hline$i 2$ \\
\hline$i 3$ \\
\hline$i 4$ \\
\hline$i 5$ \\
\hline$i 6$ \\
\hline$i 7$ \\
\hline$i 8$ \\
\hline$i 9$ \\
\hline$i 10$ \\
\hline$i 11$ \\
\hline$i 12$ \\
\hline$i 13$ \\
\hline$i 14$ \\
\hline$i 15$ \\
\hline$i 16$ \\
\hline
\end{tabular}

\begin{tabular}{|c|r|r|}
\hline \multicolumn{3}{|c|}{ Measured Values } \\
\hline 01 & \multicolumn{1}{|c|}{02} & \multicolumn{1}{|c|}{03} \\
\hline 0.16655 & 0.502 & 1 \\
\hline 0.16606 & 0.5022 & 1 \\
\hline 0.16805 & 0.50247 & 1 \\
\hline 0.50868 & 0.13002 & 0.83719 \\
\hline 0.72533 & 0.17241 & 0.3431 \\
\hline 0.50562 & 0.13304 & 0.83642 \\
\hline 0.8234 & 0.18662 & 0.50348 \\
\hline 0.8215 & 0.19259 & 0.50068 \\
\hline 0.83189 & 0.50225 & 0.16396 \\
\hline 0.50542 & 0.13934 & 0.83827 \\
\hline 0.83184 & 0.17632 & 0.50106 \\
\hline 0.84429 & 0.4976 & 0.16474 \\
\hline 0.84295 & 0.49853 & 0.16853 \\
\hline 0.82804 & 0.18079 & 0.50192 \\
\hline 0.83371 & 0.5024 & 0.1698 \\
\hline 0.83195 & 0.50208 & 0.16326 \\
\hline
\end{tabular}
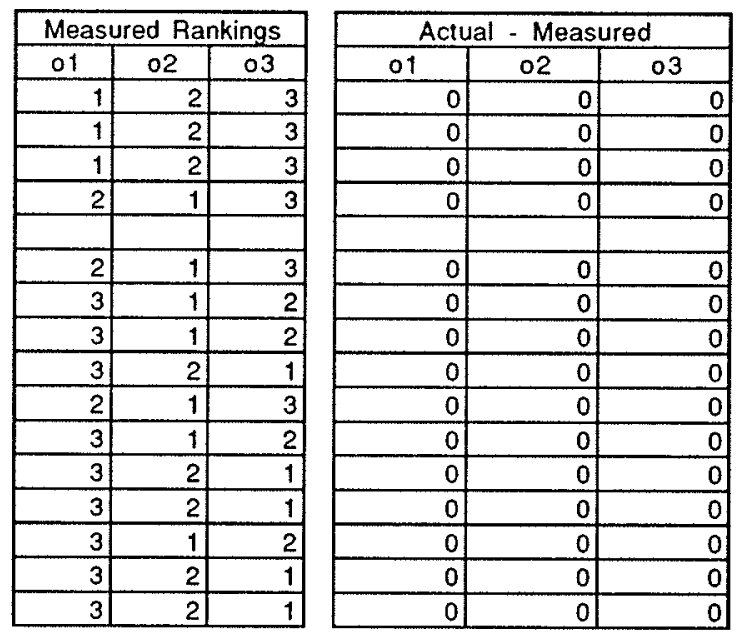

Page C.5 
Routing Results for Node 6

of the Sample Network

\begin{tabular}{|c|c|c|c|c|c|}
\hline \multirow{2}{*}{$\begin{array}{l}\text { Destination } \\
\text { Node }\end{array}$} & \multicolumn{5}{|c|}{ Real Output Values } \\
\hline & 01 & 02 & 03 & 04 & 05 \\
\hline i1 & 0.38915 & 0.26626 & 0.40625 & 0.59828 & 0.72821 \\
\hline 12 & 0.1573 & 0.23351 & 0.38872 & 0.58075 & 0.71068 \\
\hline 13 & 0.25685 & 0.13396 & 0.28917 & 0.4812 & 0.61113 \\
\hline$i 4$ & 0.48087 & 0.35798 & 25 & 0.45628 & 0.58621 \\
\hline$i 5$ & 0.54695 & 0.42406 & 0.36006 & 0.55209 & 0.68202 \\
\hline i6 & & & & & \\
\hline$i 7$ & 0.38132 & 0.25843 & 0.1647 & 0.35673 & 0.48666 \\
\hline$i 8$ & 0.49108 & 0.36819 & 0.27446 & 0.39187 & 0.5218 \\
\hline i9 & 0.59418 & 0.47129 & 0.37756 & 0.49497 & 0.59212 \\
\hline$i 10$ & 0.59418 & 0.47129 & 0.37756 & 0.14387 & 0.35647 \\
\hline$i 11$ & 0.49108 & 0.36819 & 0.27446 & 0.24697 & 0.3769 \\
\hline i12 & 0.60995 & 0.48706 & 0.39333 & 0.36584 & 0.45072 \\
\hline i13 & 0.77075 & 0.64786 & 0.55413 & 0.52664 & 0.61152 \\
\hline$i 14$ & 0.63598 & 0.51309 & 0.41936 & 0.26834 & 0.232 \\
\hline it & 0.62658 & 0.50369 & 0.4 & 0.3773 & 0.34096 \\
\hline$i 16$ & 0.80688 & 0.68399 & 0.59026 & 0.5576 & 0.52126 \\
\hline
\end{tabular}
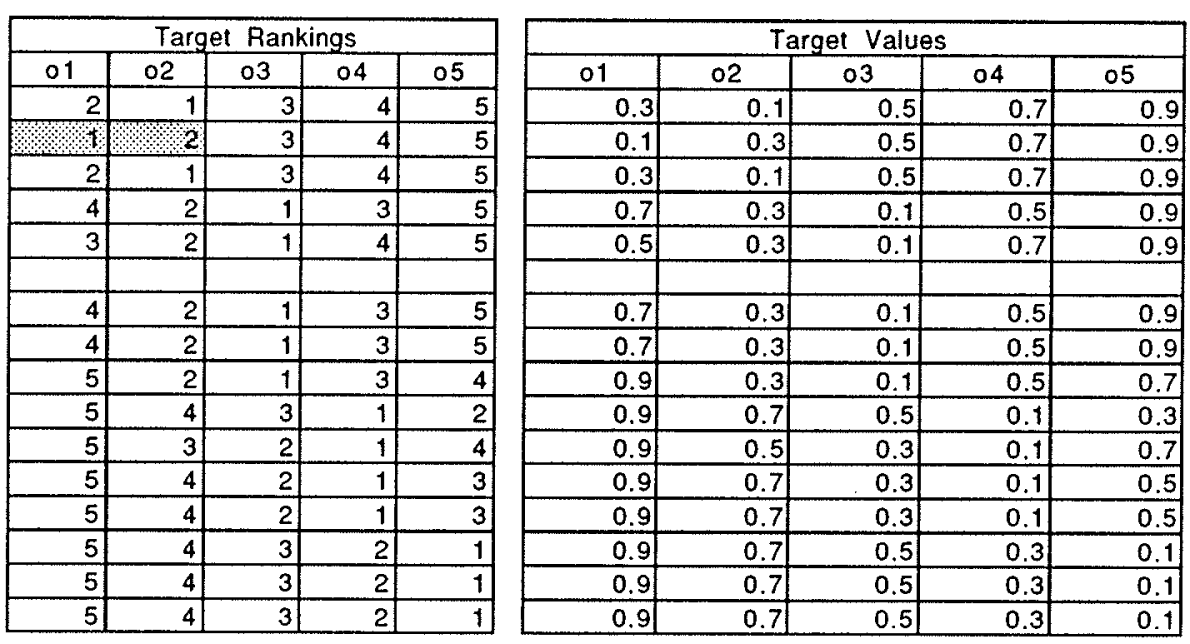

\begin{tabular}{|c|}
\hline $\begin{array}{c}\text { Destination } \\
\text { Node }\end{array}$ \\
\hline 11 \\
\hline$i 2$ \\
\hline$i 3$ \\
\hline$i 4$ \\
\hline$i 5$ \\
\hline$i 6$ \\
\hline$i 7$ \\
\hline 18 \\
\hline$i 9$ \\
\hline 110 \\
\hline$i 11$ \\
\hline$i 12$ \\
\hline$i 13$ \\
\hline$i 14$ \\
\hline$i 15$ \\
\hline$i 16$ \\
\hline
\end{tabular}

\begin{tabular}{|r|r|r|r|r|}
\hline \multicolumn{6}{|c|}{ Measured Values } \\
\hline 01 & \multicolumn{1}{|c|}{02} & \multicolumn{1}{|c|}{03} & \multicolumn{1}{c|}{04} & \multicolumn{1}{c|}{05} \\
\hline 0.25232 & 0.19123 & 0.47996 & 0.70413 & 0.90557 \\
\hline 0.18201 & 0.16575 & 0.52827 & 0.6938 & 0.88846 \\
\hline 0.25292 & 0.18943 & 0.4806 & 0.6989 & 0.9185 \\
\hline 0.7113 & 0.28848 & 0.10273 & 0.50243 & 0.89146 \\
\hline 0.53027 & 0.2118 & 0.13418 & 0.69561 & 0.90745 \\
\hline 0.18339 & 0.08281 & 0.0428 & 0.05672 & 0.11014 \\
\hline 0.70417 & 0.29037 & 0.11097 & 0.49872 & 0.90084 \\
\hline 0.7061 & 0.29221 & 0.11157 & 0.50426 & 0.88712 \\
\hline 0.85164 & 0.34563 & 0.04953 & 0.49798 & 0.70498 \\
\hline 0.91785 & 0.71056 & 0.48507 & 0.1055 & 0.29382 \\
\hline 0.8596 & 0.54984 & 0.26405 & 0.13165 & 0.69051 \\
\hline 0.91634 & 0.67102 & 0.32213 & 0.0641 & 0.50751 \\
\hline 0.92207 & 0.67397 & 0.31777 & 0.07659 & 0.50421 \\
\hline 0.9005 & 0.69775 & 0.50105 & 0.30207 & 0.09311 \\
\hline 0.90131 & 0.69584 & 0.50291 & 0.30099 & 0.102 \\
\hline 0.90402 & 0.69717 & 0.50045 & 0.29798 & 0.10287 \\
\hline & & & & \\
\hline
\end{tabular}
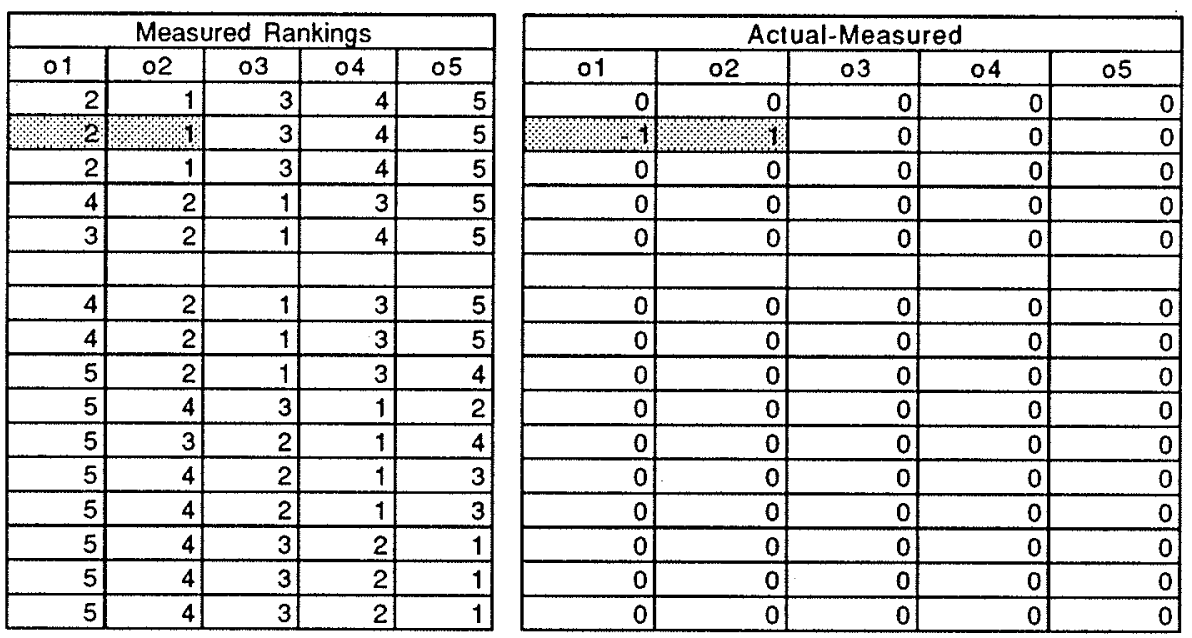

Page C.6 
Routing Results for Node 7

of the Sample Network

\begin{tabular}{|c|c|c|c|c|c|}
\hline \multirow{2}{*}{$\begin{array}{c}\text { Destination } \\
\text { Node }\end{array}$} & \multicolumn{5}{|c|}{ Real Output Values } \\
\hline & 01 & 02 & 03 & 04 & 05 \\
\hline i1 & 0.28177 & 0.26655 & 0.40596 & 0.39043 & 0.5353 \\
\hline$i 2$ & 0.24902 & 0.4984 & 0.297 & 0.62228 & 0.48903 \\
\hline$i 3$ & 0.14947 & 0.39885 & 0.27366 & 0.52273 & 0.46569 \\
\hline 14 & 0.42377 & 0.12455 & 0.5 & 0.24843 & 0.39333 \\
\hline$i 5$ & 0.43957 & 0.22036 & 0.56376 & 0.34424 & 0.48914 \\
\hline$i 6$ & 0.25843 & 0.50781 & .1647 & 0.50163 & 0.35673 \\
\hline$i 7$ & & & & & \\
\hline 18 & 0.53744 & 0.23822 & 0.53157 & 0.13476 & 0.27966 \\
\hline 19 & 0.62756 & 0.34132 & 0.63467 & 0.23786 & 0.38276 \\
\hline 110 & 0.3773 & 0.48622 & 0.28357 & 0.38276 & 0.23786 \\
\hline$i 11$ & 0.4804 & 0.38312 & 0.38667 & 0.27966 & 0.13476 \\
\hline$i 12$ & 0.59927 & 0.41462 & 0.50554 & 0.31116 & 0.25363 \\
\hline i13 & 0.76007 & 0.55402 & 0.66634 & 0.45056 & 0.41443 \\
\hline$i 14$ & 0.46543 & 0.52802 & 0.3717 & 0.42456 & 0.27966 \\
\hline$i 15$ & 0.57439 & 0.51862 & 0.48066 & 0.42092 & 0.27026 \\
\hline$i 16$ & 0.75469 & 0.62032 & 0.66096 & 0.51686 & 0.45056 \\
\hline
\end{tabular}
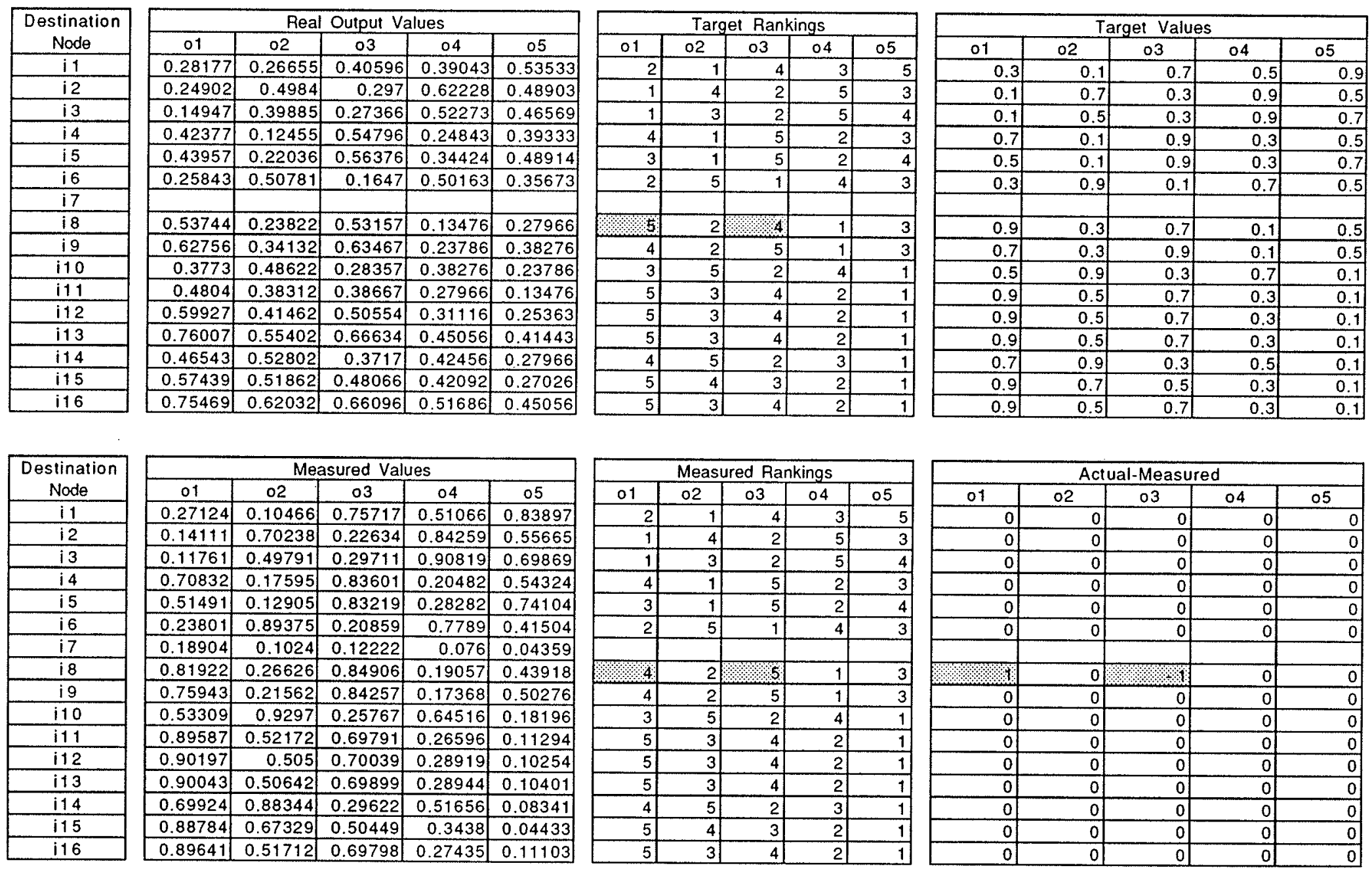

Page C.7 
Routing Results for Node 8

of the Sample Network

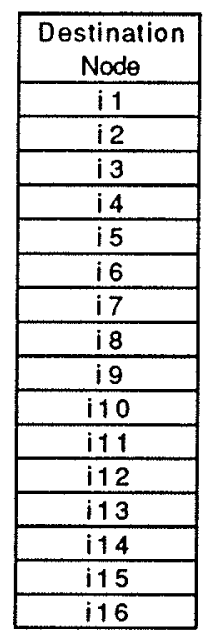

\begin{tabular}{|c|c|r|r|r|}
\hline \multicolumn{6}{|c|}{ Real Output Values } \\
\hline 01 & \multicolumn{1}{|c|}{02} & \multicolumn{1}{|c|}{03} & \multicolumn{1}{|c|}{04} & \multicolumn{1}{c|}{05} \\
\hline 0.28067 & 0.37631 & 0.4739 & 0.52121 & 0.67158 \\
\hline 0.46224 & 0.35878 & 0.70574 & 0.50368 & 0.65405 \\
\hline 0.36269 & 0.25923 & 0.6062 & 0.40413 & 0.5545 \\
\hline 0.13867 & 0.23431 & 0.41191 & 0.37921 & 0.52958 \\
\hline 0.23448 & 0.33012 & 0.3161 & 0.47502 & 0.5308 \\
\hline 0.37792 & 0.27446 & 0.61034 & 0.39187 & 0.54224 \\
\hline 0.23822 & 0.13476 & 0.49813 & 0.27966 & 0.43003 \\
\hline & & & & \\
\hline 0.42248 & 0.42003 & 0.1281 & 0.43017 & 0.3428 \\
\hline 0.45108 & 0.34762 & 0.49147 & 0.273 & 0.42337 \\
\hline 0.34798 & 0.24452 & 0.38837 & 0.1699 & 0.32027 \\
\hline 0.46685 & 0.36339 & 0.2695 & 0.28877 & 0.2014 \\
\hline 0.62765 & 0.52419 & 0.3408 & 0.44957 & 0.3622 \\
\hline 0.49288 & 0.38942 & 0.48822 & 0.3148 & 0.42012 \\
\hline 0.48348 & 0.38002 & 0.37926 & 0.3054 & 0.31116 \\
\hline 0.66378 & 0.56032 & 0.4752 & 0.4857 & 0.4071 \\
\hline
\end{tabular}
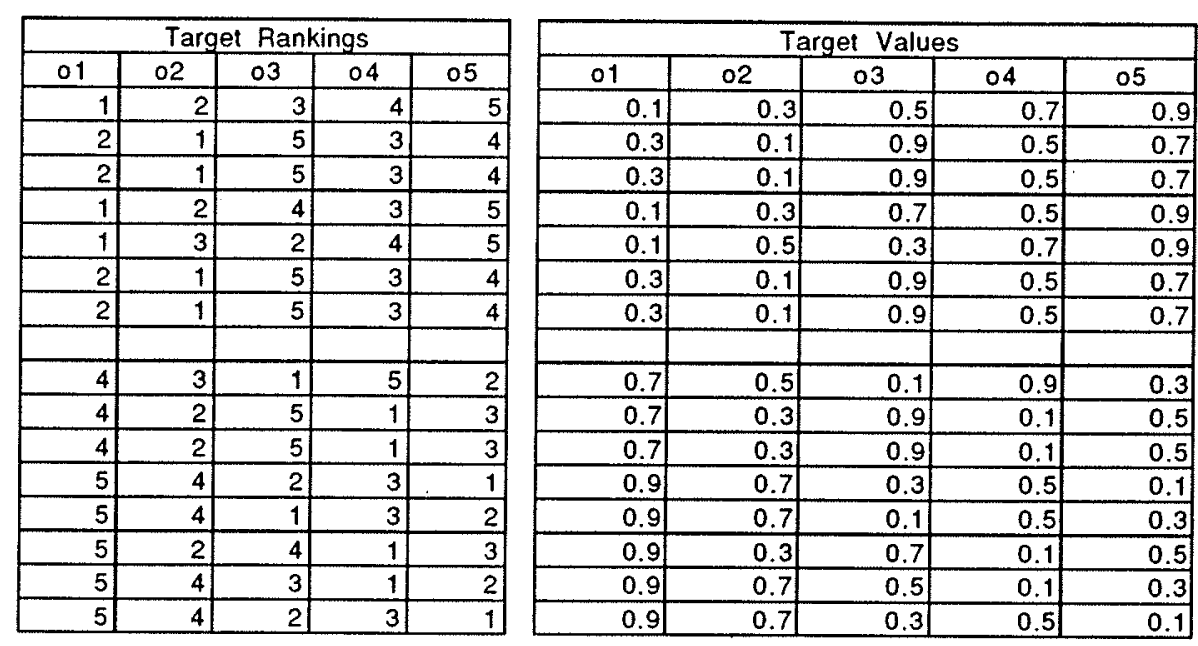

\begin{tabular}{|c|}
\hline $\begin{array}{c}\text { Destination } \\
\text { Node }\end{array}$ \\
\hline$i 1$ \\
\hline$i 2$ \\
\hline$i 3$ \\
\hline$i 4$ \\
\hline$i 5$ \\
\hline$i 6$ \\
\hline$i 7$ \\
\hline$i 8$ \\
\hline$i 9$ \\
\hline$i 10$ \\
\hline$i 11$ \\
\hline$i 12$ \\
\hline$i 13$ \\
\hline$i 14$ \\
\hline$i 15$ \\
\hline$i 16$ \\
\hline
\end{tabular}
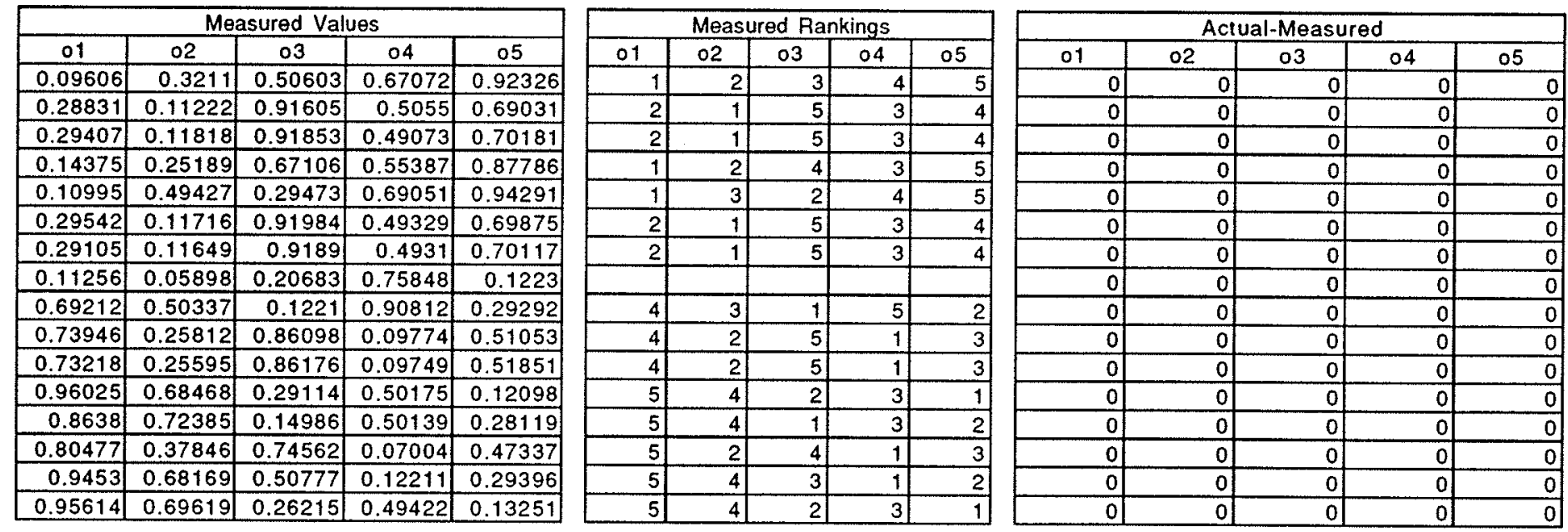

Page C.8 
Routing Results for Node 9 of the Sample Network

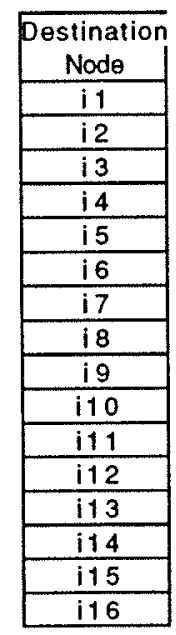

\begin{tabular}{|c|c|c|c|}
\hline \multicolumn{4}{|c|}{ Real Output Values } \\
\hline 01 & 02 & 03 & 04 \\
\hline 0.3708 & 0.3838 & 0.5985 & 0.8306 \\
\hline 0.6027 & 0.4619 & 0.6191 & 0.8512 \\
\hline 0.5031 & 0.3623 & 0.5195 & 0.7516 \\
\hline 0.3088 & 0.2418 & 0.4565 & 0.6886 \\
\hline 0.213 & 0.3376 & 0.5523 & 0.7844 \\
\hline 0.5481 & 0.3776 & 0.5072 & 0.7393 \\
\hline 0.4084 & 0.2379 & 0.395 & 0.6271 \\
\hline 0.4225 & 0.1281 & 0.3428 & 0.5749 \\
\hline & & & \\
\hline 0.6212 & 0.3761 & 0.3884 & 0.6205 \\
\hline 0.5181 & 0.273 & 0.2853 & 0.5174 \\
\hline 0.5989 & 0.3045 & 0.1664 & 0.3985 \\
\hline 0.7597 & 0.4653 & 0.3272 & 0.2377 \\
\hline 0.663 & 0.4179 & 0.3851 & 0.6172 \\
\hline 0.6536 & 0.4085 & 0.2762 & 0.5083 \\
\hline 0.8046 & 0.5102 & 0.3712 & 0.3929 \\
\hline
\end{tabular}

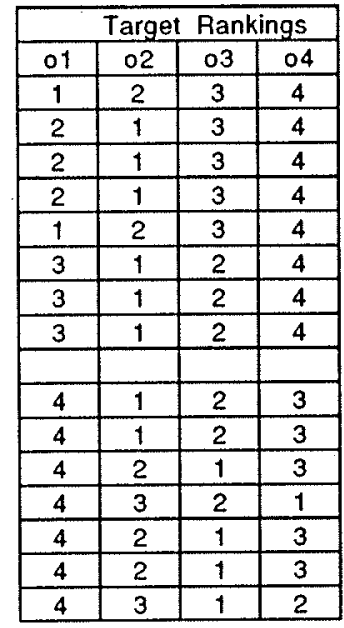

\begin{tabular}{|l|l|l|l|}
\hline \multicolumn{4}{|c|}{ Target Values } \\
\hline 01 & 02 & 03 & 04 \\
\hline 0.125 & 0.375 & 0.625 & 0.875 \\
\hline 0.375 & 0.125 & 0.625 & 0.875 \\
\hline 0.375 & 0.125 & 0.625 & 0.875 \\
\hline 0.375 & 0.125 & 0.625 & 0.875 \\
\hline 0.125 & 0.375 & 0.625 & 0.875 \\
\hline 0.625 & 0.125 & 0.375 & 0.875 \\
\hline 0.625 & 0.125 & 0.375 & 0.875 \\
\hline 0.625 & 0.125 & 0.375 & 0.875 \\
\hline & & & \\
\hline 0.875 & 0.125 & 0.375 & 0.625 \\
\hline 0.875 & 0.125 & 0.375 & 0.625 \\
\hline 0.875 & 0.375 & 0.125 & 0.625 \\
\hline 0.875 & 0.625 & 0.375 & 0.125 \\
\hline 0.875 & 0.375 & 0.125 & 0.625 \\
\hline 0.875 & 0.375 & 0.125 & 0.625 \\
\hline 0.875 & 0.625 & 0.125 & 0.375 \\
\hline
\end{tabular}

\begin{tabular}{|c|}
\hline $\begin{array}{c}\text { Destination } \\
\text { Node }\end{array}$ \\
\hline$i 1$ \\
\hline$i 2$ \\
\hline$i 3$ \\
\hline$i 4$ \\
\hline$i 5$ \\
\hline$i 6$ \\
\hline$i 7$ \\
\hline$i 8$ \\
\hline$i 9$ \\
\hline$i 10$ \\
\hline$i 11$ \\
\hline$i 12$ \\
\hline$i 13$ \\
\hline$i 14$ \\
\hline$i 15$ \\
\hline$i 16$ \\
\hline
\end{tabular}
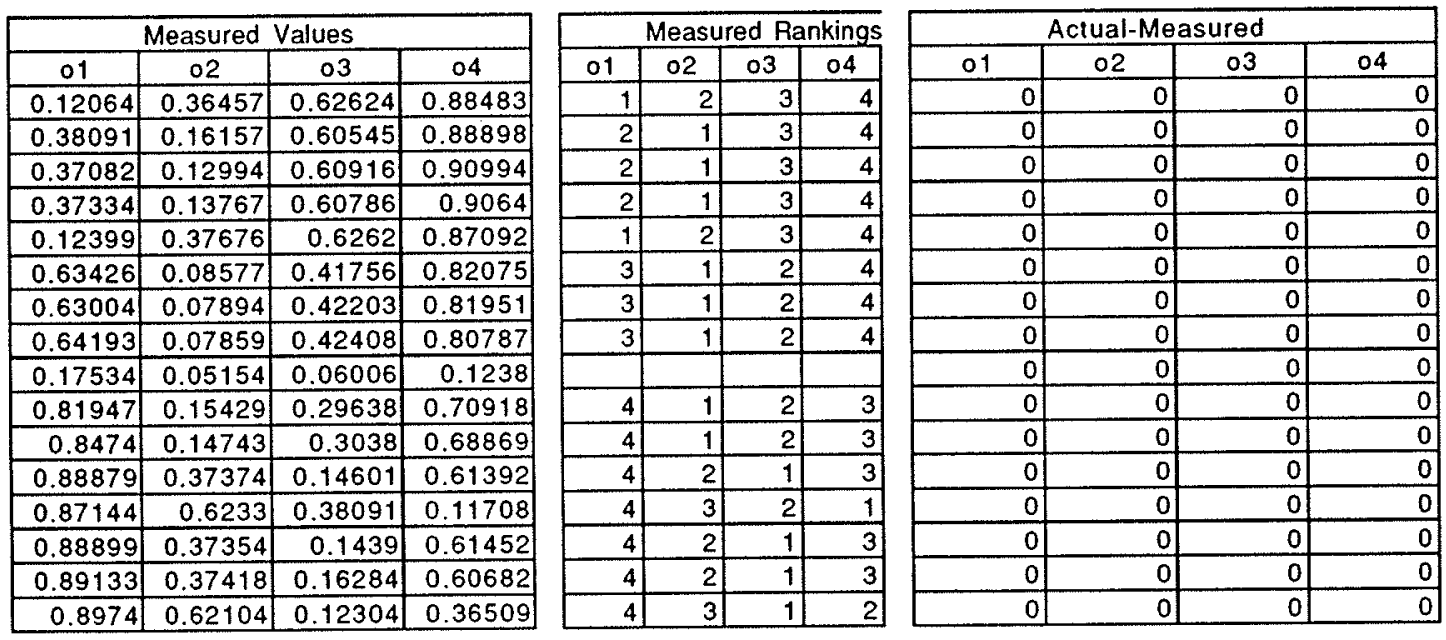

Page C.9 
Routing Results for Node 10

of the Sample Network

\begin{tabular}{|c|}
\hline $\begin{array}{c}\text { Destination } \\
\text { Node }\end{array}$ \\
\hline 11 \\
\hline 12 \\
\hline 13 \\
\hline 14 \\
\hline 15 \\
\hline 16 \\
\hline 17 \\
\hline 18 \\
\hline 19 \\
\hline 110 \\
\hline 111 \\
\hline 112 \\
\hline 113 \\
\hline 114 \\
\hline 115 \\
\hline 116 \\
\hline
\end{tabular}

\begin{tabular}{|c|c|c|}
\hline \multicolumn{3}{|c|}{ Real Output Values } \\
\hline 01 & \multicolumn{1}{c|}{02} & \multicolumn{1}{c|}{03} \\
\hline 0.38513 & 0.47941 & 0.59773 \\
\hline 0.27617 & 0.46188 & 0.48877 \\
\hline 0.25283 & 0.36233 & 0.46543 \\
\hline 0.38312 & 0.33741 & 0.50368 \\
\hline 0.47893 & 0.43322 & 0.59949 \\
\hline 0.14387 & 0.37756 & 0.35647 \\
\hline 0.28357 & 0.23786 & 0.40413 \\
\hline 0.39333 & 0.273 & 0.43927 \\
\hline 0.47643 & 0.3761 & 0.50959 \\
\hline & & \\
\hline 0.39333 & 0.1281 & 0.29437 \\
\hline 0.5122 & 0.24697 & 0.38819 \\
\hline 0.673 & 0.40777 & 0.52899 \\
\hline 0.35087 & 0.273 & 0.14947 \\
\hline 0.45983 & 0.2636 & 0.25843 \\
\hline 0.64013 & 0.4439 & 0.43873 \\
\hline
\end{tabular}
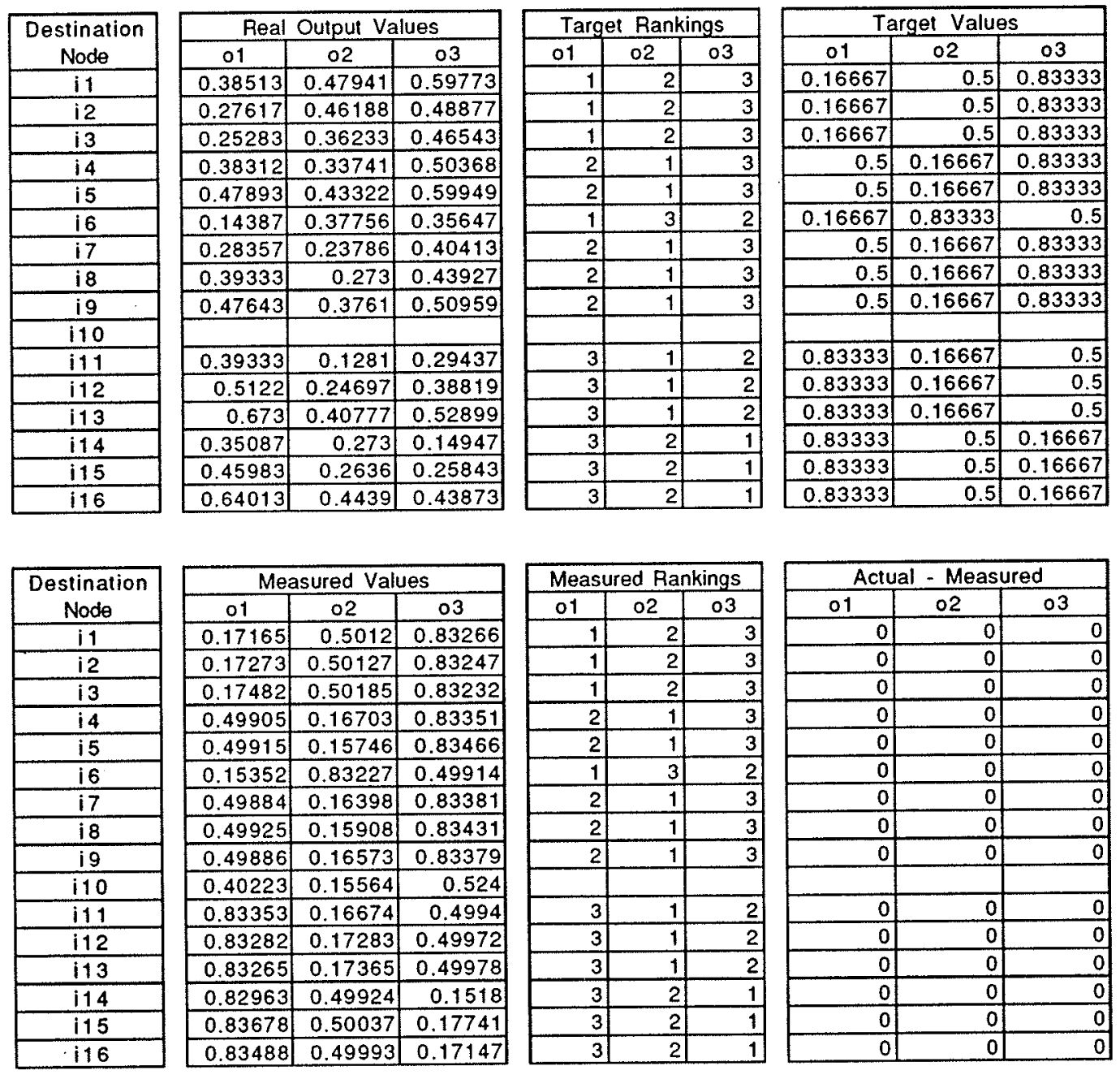

Page C.10 
Routing Results for Node 11

of the Sample Network

\begin{tabular}{|c|c|c|c|c|c|c|}
\hline \multirow{2}{*}{$\begin{array}{c}\text { Destination } \\
\text { Node }\end{array}$} & \multicolumn{6}{|c|}{ Real Output Values } \\
\hline & 01 & 02 & 03 & 04 & 05 & 06 \\
\hline$i 1$ & 0.3763 & 0.4256 & 0.4882 & 0.5759 & 0.6182 & 0.7023 \\
\hline$i 2$ & 0.3588 & 0.5037 & 0.3793 & 0.6541 & 0.5092 & 0.6088 \\
\hline i3 & 0.2592 & 0.4041 & 0.3559 & 0.5545 & 0.4859 & 0.5854 \\
\hline$i 4$ & 0.2343 & 0.2836 & 0.4862 & 0.4339 & 0.6162 & 0.5603 \\
\hline i5 & 0.3301 & 0.3794 & 0.582 & 0.4732 & 0.712 & 0.5997 \\
\hline 16 & 0.2745 & 0.4194 & 0.247 & 0.5696 & 0.3769 & 0.4765 \\
\hline 17 & 0.1348 & 0.2797 & 0.3867 & 0.43 & 0.5166 & 0.5564 \\
\hline 18 & 0.2445 & 0.1699 & 0.4964 & 0.3203 & 0.565 & 0.4467 \\
\hline i9 & 0.3476 & 0.273 & 0.5995 & 0.2853 & 0.53 & 0.4117 \\
\hline$i 10$ & 0.3933 & 0.5382 & 0.1281 & 0.4871 & 0.2944 & 0.3939 \\
\hline \multicolumn{7}{|l|}{$i 11$} \\
\hline$i 12$ & 0.4209 & 0.3463 & 0.4713 & 0.1439 & 0.3886 & 0.2703 \\
\hline$i 13$ & 0.5603 & 0.4857 & 0.6321 & 0.3047 & 0.5494 & 0.4311 \\
\hline$i 14$ & 0.4815 & 0.6331 & 0.2526 & 0.3626 & 0.1699 & 0.2695 \\
\hline$i 15$ & 0.5307 & 0.456 & 0.3615 & 0.2536 & 0.2789 & 0.1605 \\
\hline$i 16$ & 0.6266 & 0.552 & 0.5418 & 0.3496 & 0.4591 & 0.3408 \\
\hline
\end{tabular}

\begin{tabular}{|c|c|c|c|c|c|}
\hline \multicolumn{7}{|c|}{ Target Rankings } \\
\hline 01 & 02 & 03 & 04 & 05 & 06 \\
\hline 1 & 2 & 3 & 4 & 5 & 6 \\
\hline 1 & 3 & 2 & 6 & 4 & 5 \\
\hline 1 & 3 & 2 & 5 & 4 & 6 \\
\hline 1 & 2 & 4 & 3 & 6 & 5 \\
\hline 1 & 2 & 4 & 3 & 6 & 5 \\
\hline 2 & 4 & 1 & 6 & 3 & 5 \\
\hline 1 & 2 & 3 & 4 & 5 & 6 \\
\hline 2 & 1 & 5 & 3 & 6 & 4 \\
\hline 3 & 1 & 6 & 2 & 5 & 4 \\
\hline 3 & 6 & 1 & 5 & 2 & 4 \\
\hline & & & & & \\
\hline 5 & 3 & 6 & 1 & 4 & 2 \\
\hline 5 & 3 & 6 & 1 & 4 & 2 \\
\hline 5 & 6 & 2 & 4 & 1 & 3 \\
\hline 6 & 5 & 4 & 2 & 3 & 1 \\
\hline 6 & 5 & 4 & 2 & 3 & 1 \\
\hline
\end{tabular}

\begin{tabular}{|r|r|r|r|r|r|}
\hline \multicolumn{7}{|c|}{ Target Values } \\
\hline \multicolumn{1}{|c|}{01} & \multicolumn{1}{c|}{02} & \multicolumn{1}{c|}{03} & \multicolumn{1}{c|}{04} & \multicolumn{1}{c|}{05} & \multicolumn{1}{c|}{06} \\
\hline 0.08333 & 0.25 & 0.41667 & 0.58333 & 0.75 & 0.91667 \\
\hline 0.08333 & 0.41667 & 0.25 & 0.91667 & 0.58333 & 0.75 \\
\hline 0.08333 & 0.41667 & 0.25 & 0.75 & 0.58333 & 0.91667 \\
\hline 0.08333 & 0.25 & 0.58333 & 0.41667 & 0.91667 & 0.75 \\
\hline 0.08333 & 0.25 & 0.58333 & 0.41667 & 0.91667 & 0.75 \\
\hline 0.25 & 0.58333 & 0.08333 & 0.91667 & 0.41667 & 0.75 \\
\hline 0.08333 & 0.25 & 0.41667 & 0.58333 & 0.75 & 0.91667 \\
\hline 0.25 & 0.08333 & 0.75 & 0.41667 & 0.91667 & 0.58333 \\
\hline 0.41667 & 0.08333 & 0.91667 & 0.25 & 0.75 & 0.58333 \\
\hline 0.41667 & 0.91667 & 0.08333 & 0.75 & 0.25 & 0.58333 \\
\hline & & & & & \\
\hline 0.75 & 0.41667 & 0.91667 & 0.08333 & 0.58333 & 0.25 \\
\hline 0.75 & 0.41667 & 0.91667 & 0.08333 & 0.58333 & 0.25 \\
\hline 0.75 & 0.91667 & 0.25 & 0.58333 & 0.08333 & 0.41667 \\
\hline 0.91667 & 0.75 & 0.58333 & 0.25 & 0.41667 & 0.08333 \\
\hline 0.91667 & 0.75 & 0.58333 & 0.25 & 0.41667 & 0.08333 \\
\hline
\end{tabular}

\begin{tabular}{|c|}
\hline $\begin{array}{c}\text { Destination } \\
\text { Node }\end{array}$ \\
\hline$i 1$ \\
\hline$i 2$ \\
\hline$i 3$ \\
\hline$i 4$ \\
\hline$i 5$ \\
\hline$i 6$ \\
\hline$i 7$ \\
\hline$i 8$ \\
\hline$i 9$ \\
\hline$i 10$ \\
\hline$i 11$ \\
\hline$i 12$ \\
\hline$i 13$ \\
\hline$i 14$ \\
\hline$i 15$ \\
\hline$i 16$ \\
\hline
\end{tabular}
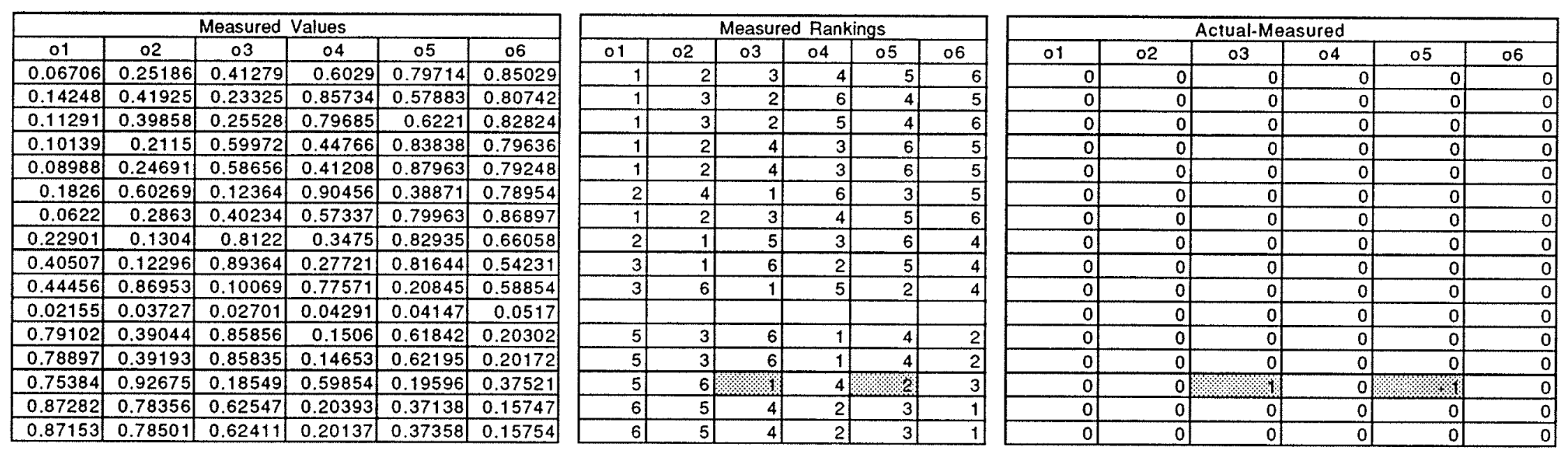

Page C.11 
Routing Results for Node 12

of the Sample Network

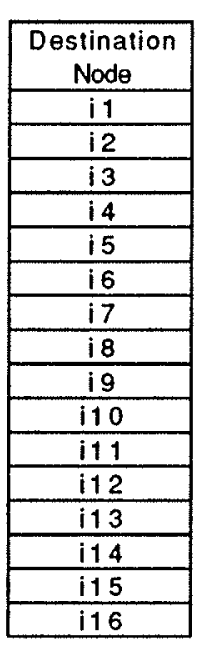

\begin{tabular}{|c|r|r|r|r|r|}
\hline \multicolumn{7}{|c|}{ Real Output Values } \\
\hline 01 & \multicolumn{1}{|c|}{02} & \multicolumn{1}{|c|}{03} & \multicolumn{1}{c|}{04} & \multicolumn{1}{c|}{05} & \multicolumn{1}{c|}{06} \\
\hline 0.45707 & 0.5122 & 0.49518 & 0.7443 & 0.61257 & 0.8058 \\
\hline 0.53518 & 0.60328 & 0.47765 & 0.83538 & 0.58302 & 0.85926 \\
\hline 0.43563 & 0.50373 & 0.3781 & 0.73583 & 0.50449 & 0.78073 \\
\hline 0.31507 & 0.38317 & 0.35318 & 0.61527 & 0.47957 & 0.75581 \\
\hline 0.41088 & 0.3544 & 0.44899 & 0.5865 & 0.57538 & 0.7866 \\
\hline 0.45086 & 0.51896 & 0.36584 & 0.75106 & 0.45072 & 0.72696 \\
\hline 0.31116 & 0.37926 & 0.25363 & 0.61136 & 0.38002 & 0.65626 \\
\hline 0.2014 & 0.2695 & 0.28877 & 0.5016 & 0.41516 & 0.6914 \\
\hline 0.3045 & 0.16664 & 0.39187 & 0.3985 & 0.51826 & 0.5986 \\
\hline 0.4494 & 0.5175 & 0.24697 & 0.7496 & 0.37336 & 0.6496 \\
\hline 0.3463 & 0.4144 & 0.14387 & 0.6465 & 0.27026 & 0.5465 \\
\hline & & & & & \\
\hline 0.5172 & 0.3791 & 0.60457 & 0.1858 & 0.47026 & 0.3859 \\
\hline 0.4912 & 0.5593 & 0.28877 & 0.63026 & 0.24372 & 0.51996 \\
\hline 0.4818 & 0.5499 & 0.27937 & 0.5213 & 0.13476 & 0.411 \\
\hline 0.6621 & 0.5343 & 0.45967 & 0.341 & 0.31506 & 0.2307 \\
\hline
\end{tabular}

\begin{tabular}{|c|}
\hline $\begin{array}{c}\text { Destination } \\
\text { Node }\end{array}$ \\
\hline$i 1$ \\
\hline$i 2$ \\
\hline$i 3$ \\
\hline$i 4$ \\
\hline$i 5$ \\
\hline$i 6$ \\
\hline$i 7$ \\
\hline$i 8$ \\
\hline$i 9$ \\
\hline$i 10$ \\
\hline$i 11$ \\
\hline$i 12$ \\
\hline$i 13$ \\
\hline$i 14$ \\
\hline$i 15$ \\
\hline$i 16$ \\
\hline
\end{tabular}

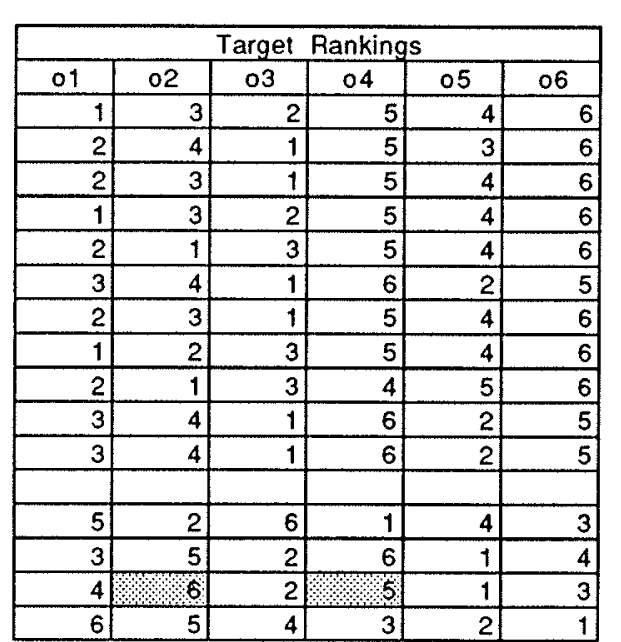

\begin{tabular}{|r|r|r|r|r|r|}
\hline \multicolumn{7}{|c|}{ Target Values } \\
\hline 01 & \multicolumn{1}{|c|}{02} & \multicolumn{1}{c|}{03} & \multicolumn{1}{c|}{04} & \multicolumn{1}{c|}{05} & \multicolumn{1}{c|}{06} \\
\hline 0.08333 & 0.41667 & 0.25 & 0.75 & 0.58333 & 0.91667 \\
\hline 0.25 & 0.58333 & 0.08333 & 0.75 & 0.41667 & 0.91667 \\
\hline 0.25 & 0.41667 & 0.08333 & 0.75 & 0.58333 & 0.91667 \\
\hline 0.08333 & 0.41667 & 0.25 & 0.75 & 0.58333 & 0.91667 \\
\hline 0.25 & 0.08333 & 0.41667 & 0.75 & 0.58333 & 0.91667 \\
\hline 0.41667 & 0.58333 & 0.08333 & 0.91667 & 0.25 & 0.75 \\
\hline 0.25 & 0.41667 & 0.08333 & 0.75 & 0.58333 & 0.91667 \\
\hline 0.08333 & 0.25 & 0.41667 & 0.75 & 0.58333 & 0.91667 \\
\hline 0.25 & 0.08333 & 0.41667 & 0.58333 & 0.75 & 0.91667 \\
\hline 0.41667 & 0.58333 & 0.08333 & 0.91667 & 0.25 & 0.75 \\
\hline 0.41667 & 0.58333 & 0.08333 & 0.91667 & 0.25 & 0.75 \\
\hline & & & & & \\
\hline 0.75 & 0.25 & 0.91667 & 0.08333 & 0.58333 & 0.41667 \\
\hline 0.41667 & 0.75 & 0.25 & 0.91667 & 0.08333 & 0.58333 \\
\hline 0.58333 & 0.91667 & 0.25 & 0.75 & 0.08333 & 0.41667 \\
\hline 0.91667 & 0.75 & 0.58333 & 0.41667 & 0.25 & 0.08333 \\
\hline
\end{tabular}
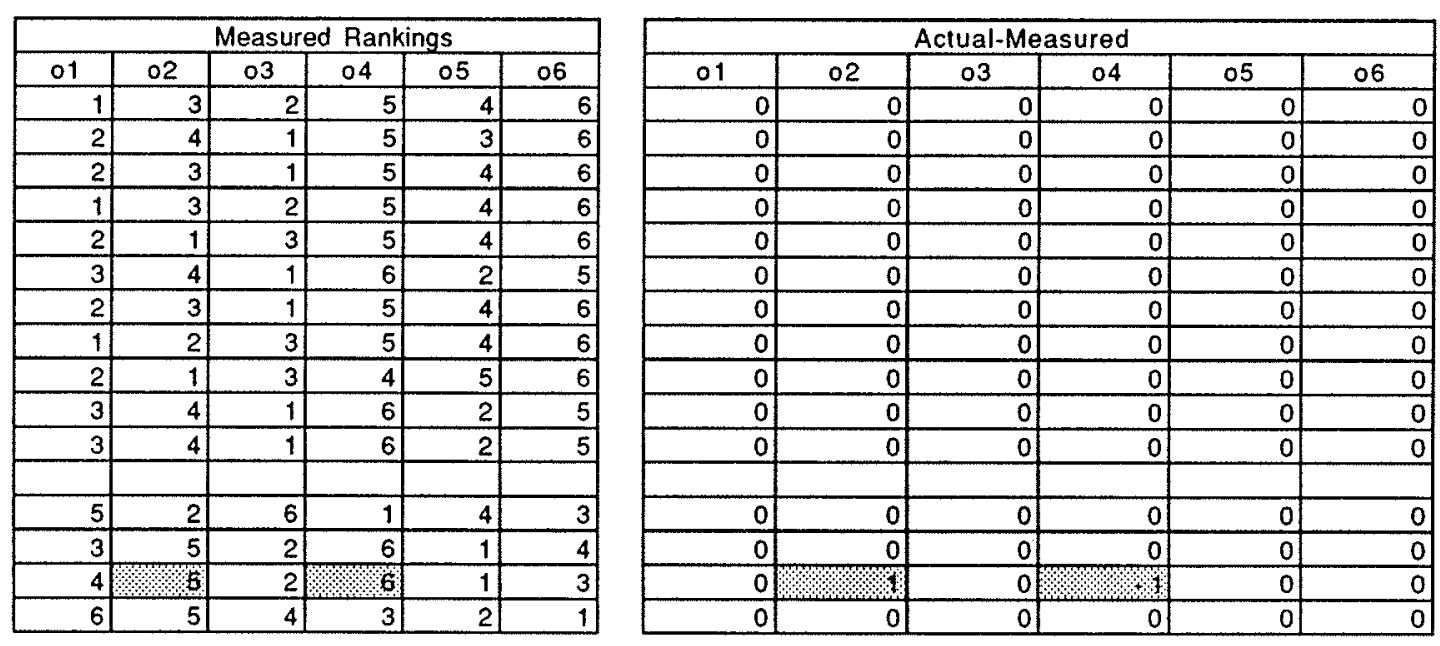
Routing Results for Node 13

of the Sample Network

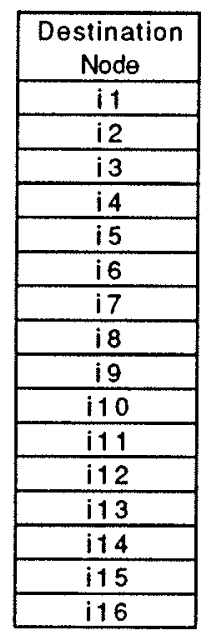

\begin{tabular}{|r|r|r|}
\hline \multicolumn{3}{|c|}{ Real Output Values } \\
\hline \multicolumn{1}{|c|}{$\mid{ }^{1}$} & \multicolumn{1}{c|}{02} & \multicolumn{1}{c|}{03} \\
\hline 0.59647 & 0.61787 & 0.7553 \\
\hline 0.67458 & 0.63845 & 0.80876 \\
\hline 0.57503 & 0.5389 & 0.73023 \\
\hline 0.45447 & 0.47587 & 0.67597 \\
\hline 0.4257 & 0.5152 & 0.7153 \\
\hline 0.59026 & 0.52664 & 0.67646 \\
\hline 0.45056 & 0.41443 & 0.60576 \\
\hline 0.3408 & 0.3622 & 0.5623 \\
\hline 0.2377 & 0.3272 & 0.5273 \\
\hline 0.5888 & 0.40777 & 0.59393 \\
\hline 0.4857 & 0.30467 & 0.496 \\
\hline 0.3791 & 0.1858 & 0.3859 \\
\hline & & \\
\hline 0.59782 & 0.40452 & 0.46946 \\
\hline 0.48886 & 0.29556 & 0.3605 \\
\hline 0.5848 & 0.3915 & 0.1802 \\
\hline
\end{tabular}

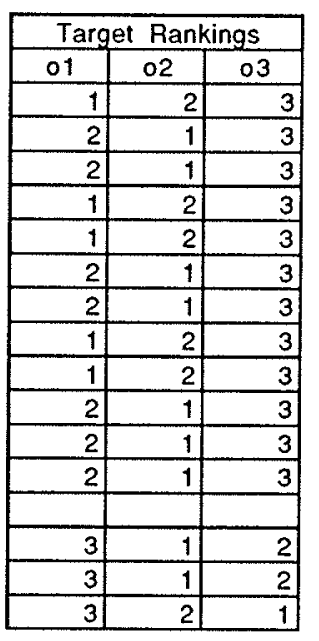

\begin{tabular}{|r|r|r|}
\hline \multicolumn{3}{|c|}{ Target Values } \\
\hline \multicolumn{1}{|c|}{01} & \multicolumn{1}{c|}{02} & \multicolumn{1}{c|}{03} \\
\hline 0.16667 & 0.5 & 0.83333 \\
\hline 0.5 & 0.16667 & 0.83333 \\
\hline 0.5 & 0.16667 & 0.83333 \\
\hline 0.16667 & 0.5 & 0.83333 \\
\hline 0.16667 & 0.5 & 0.83333 \\
\hline 0.5 & 0.16667 & 0.83333 \\
\hline 0.5 & 0.16667 & 0.83333 \\
\hline 0.16667 & 0.5 & 0.83333 \\
\hline 0.16667 & 0.5 & 0.83333 \\
\hline 0.5 & 0.16667 & 0.83333 \\
\hline 0.5 & 0.16667 & 0.83333 \\
\hline 0.5 & 0.16667 & 0.83333 \\
\hline & & \\
\hline 0.83333 & 0.16667 & 0.5 \\
\hline 0.83333 & 0.16667 & 0.5 \\
\hline 0.83333 & 0.5 & 0.16667 \\
\hline
\end{tabular}
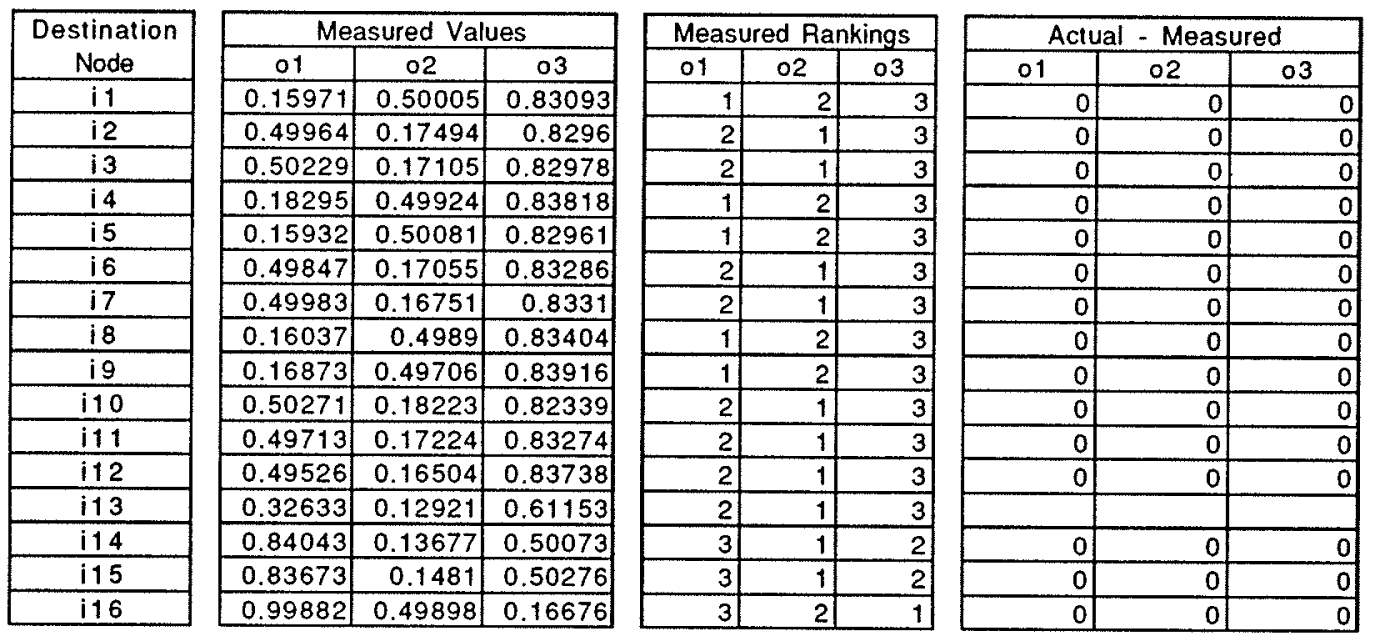

Page 0.13 
Routing Results for Node 14

of the Sample Network

\begin{tabular}{|c|c|c|c|c|}
\hline \multirow{2}{*}{$\begin{array}{l}\text { Destination } \\
\text { Node }\end{array}$} & \multicolumn{4}{|c|}{ Real Output Values } \\
\hline & 01 & 02 & 03 & 04 \\
\hline i1 & 0.47326 & 0.5096 & 0.53643 & 0.636 \\
\hline$i 2$ & 0.3643 & 0.40064 & 0.50368 & 0.60324 \\
\hline$i 3$ & 0.34096 & 0.3773 & 0.40413 & 0.50369 \\
\hline i4 & 0.47125 & 0.46188 & 0.37921 & 0.47877 \\
\hline i5 & 0.56706 & 0.55769 & 0.47502 & 0.57312 \\
\hline$i 6$ & 0.232 & 0.26834 & 0.39187 & 0.49143 \\
\hline 17 & 0.3717 & 0.36233 & 0.27966 & 0.37922 \\
\hline 18 & 0.48146 & 0.39747 & 0.3148 & 0.41436 \\
\hline 19 & 0.58456 & 0.50057 & 0.4179 & 0.38512 \\
\hline 110 & 0.35087 & 0.14947 & 0.273 & 0.37256 \\
\hline$i 11$ & 0.45399 & 0.25257 & 0.1699 & 0.36946 \\
\hline 112 & 0.57284 & 0.37144 & 0.2877 & 0.24372 \\
\hline 113 & 0.73364 & 0.53224 & 0.44957 & 0.40452 \\
\hline i 14 & & & & \\
\hline i15 & 0.58947 & 0.38807 & 0.3054 & 0.13396 \\
\hline$i 16$ & 0.76977 & 0.56837 & 0.4857 & 0.31426 \\
\hline
\end{tabular}

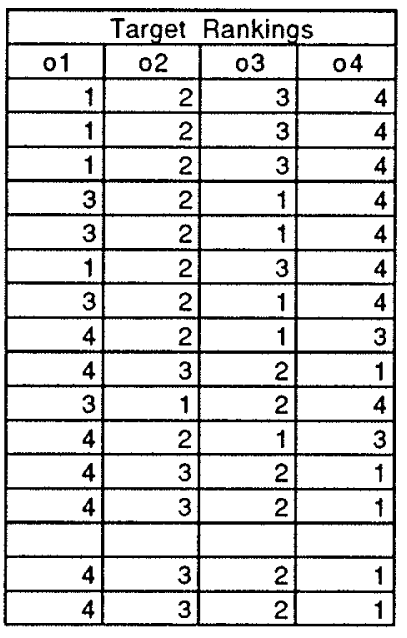

\begin{tabular}{|l|l|l|l|}
\hline \multicolumn{4}{|c|}{ Target Values } \\
\hline 01 & 02 & 03 & 04 \\
\hline 0.125 & 0.375 & 0.625 & 0.875 \\
\hline 0.125 & 0.375 & 0.625 & 0.875 \\
\hline 0.125 & 0.375 & 0.625 & 0.875 \\
\hline 0.625 & 0.375 & 0.125 & 0.875 \\
\hline 0.625 & 0.375 & 0.125 & 0.875 \\
\hline 0.125 & 0.375 & 0.625 & 0.875 \\
\hline 0.625 & 0.375 & 0.125 & 0.875 \\
\hline 0.875 & 0.375 & 0.125 & 0.625 \\
\hline 0.875 & 0.625 & 0.375 & 0.125 \\
\hline 0.625 & 0.125 & 0.375 & 0.875 \\
\hline 0.875 & 0.375 & 0.125 & 0.625 \\
\hline 0.875 & 0.625 & 0.375 & 0.125 \\
\hline 0.875 & 0.625 & 0.375 & 0.125 \\
\hline & & & \\
\hline 0.875 & 0.625 & 0.375 & 0.125 \\
\hline 0.875 & 0.625 & 0.375 & 0.125 \\
\hline
\end{tabular}

\begin{tabular}{|c|}
\hline $\begin{array}{c}\text { Destination } \\
\text { Node }\end{array}$ \\
\hline$i 1$ \\
\hline$i 2$ \\
\hline$i 3$ \\
\hline$i 4$ \\
\hline$i 5$ \\
\hline$i 6$ \\
\hline$i 7$ \\
\hline$i 8$ \\
\hline$i 9$ \\
\hline$i 10$ \\
\hline$i 11$ \\
\hline$i 12$ \\
\hline$i 13$ \\
\hline$i 14$ \\
\hline$i 15$ \\
\hline$i 16$ \\
\hline
\end{tabular}

\begin{tabular}{|c|c|c|c|}
\hline \multicolumn{4}{|c|}{ Measured Values } \\
\hline 01 & \multicolumn{1}{|c|}{02} & \multicolumn{1}{|c|}{03} & 04 \\
\hline 0.12048 & 0.37027 & 0.61759 & 0.90257 \\
\hline 0.12051 & 0.37001 & 0.61782 & 0.90249 \\
\hline 0.12089 & 0.37109 & 0.61672 & 0.90276 \\
\hline 0.62766 & 0.3819 & 0.14226 & 0.85196 \\
\hline 0.63177 & 0.37303 & 0.15155 & 0.85162 \\
\hline 0.12059 & 0.36995 & 0.61783 & 0.90254 \\
\hline 0.62758 & 0.38183 & 0.14053 & 0.85316 \\
\hline 0.87584 & 0.37207 & 0.08853 & 0.63509 \\
\hline 0.87308 & 0.62673 & 0.37411 & 0.12522 \\
\hline 0.62701 & 0.16415 & 0.40707 & 0.81003 \\
\hline 0.87068 & 0.37382 & 0.0891 & 0.63645 \\
\hline 0.87838 & 0.6183 & 0.37841 & 0.13178 \\
\hline 0.87516 & 0.62438 & 0.37473 & 0.12754 \\
\hline 0.02241 & 0.01807 & 0.01799 & 0.02124 \\
\hline 0.8735 & 0.6259 & 0.37462 & 0.12579 \\
\hline 0.87301 & 0.62685 & 0.37404 & 0.12515 \\
\hline
\end{tabular}
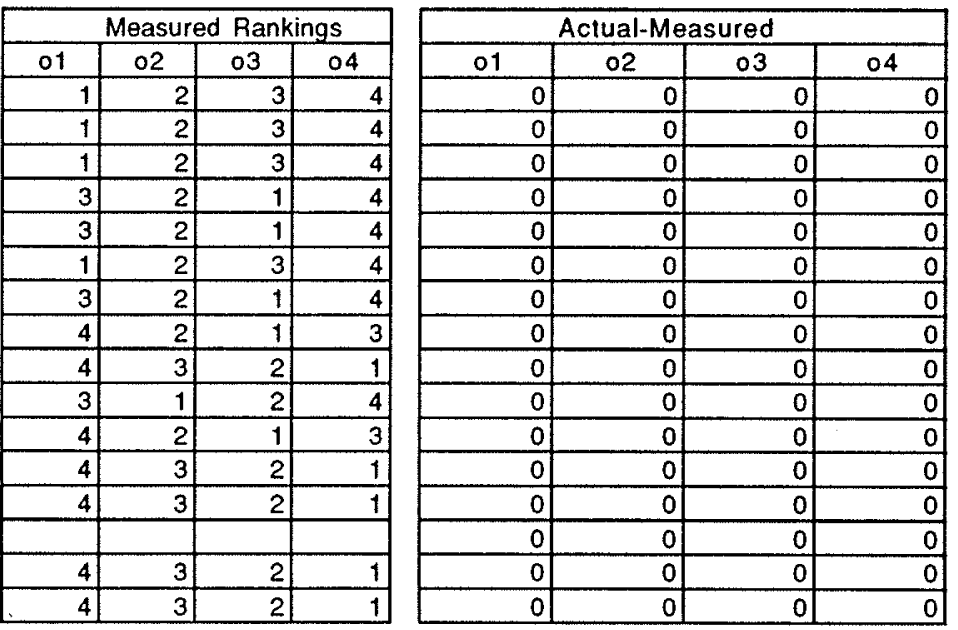

Page C.14 
Routing Results for Node 15

of the Sample Network

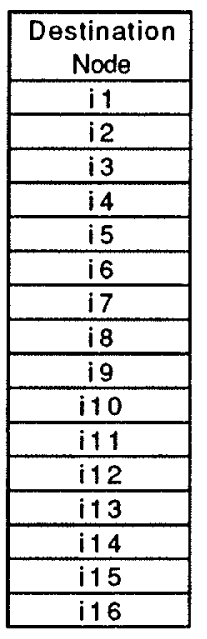

\begin{tabular}{|c|c|c|r|}
\hline \multicolumn{4}{|c|}{ Real Outpul Values } \\
\hline 01 & \multicolumn{1}{|c|}{02} & \multicolumn{1}{|c|}{03} & \multicolumn{1}{c|}{04} \\
\hline 0.51181 & 0.56683 & 0.58222 & \multicolumn{1}{c|}{0.7804} \\
\hline 0.49428 & 0.58741 & 0.47326 & 0.86365 \\
\hline 0.39473 & 0.48786 & 0.44992 & 0.7641 \\
\hline 0.36981 & 0.42483 & 0.48817 & 0.70107 \\
\hline 0.46562 & 0.46416 & 0.58398 & 0.7404 \\
\hline 0.38247 & 0.4756 & 0.34096 & 0.75184 \\
\hline 0.27026 & 0.36339 & 0.38862 & 0.63963 \\
\hline 0.3054 & 0.3116 & 0.42376 & 0.5874 \\
\hline 0.4085 & 0.27616 & 0.52686 & 0.5524 \\
\hline 0.2636 & 0.35673 & 0.25843 & 0.63297 \\
\hline 0.1605 & 0.25363 & 0.27886 & 0.52987 \\
\hline 0.27937 & 0.13476 & 0.39775 & 0.411 \\
\hline 0.44017 & 0.29556 & 0.55853 & 0.3605 \\
\hline 0.3054 & 0.39853 & 0.13396 & 0.67477 \\
\hline & & & \\
\hline 0.48507 & 0.34046 & 0.60343 & 0.2053 \\
\hline
\end{tabular}

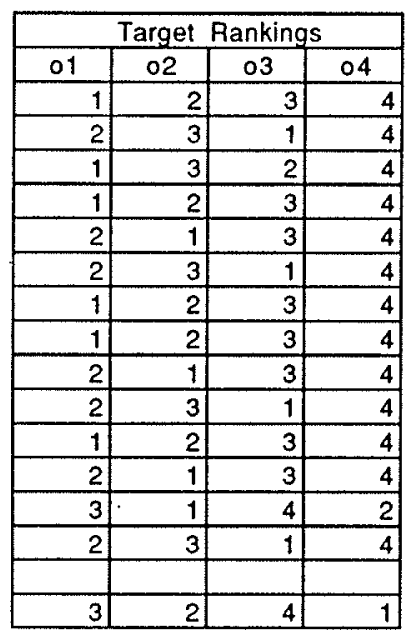

\begin{tabular}{|l|l|l|l|}
\hline \multicolumn{4}{|c|}{ Target Values } \\
\hline 01 & 02 & 03 & 04 \\
\hline 0.125 & 0.375 & 0.625 & 0.875 \\
\hline 0.375 & 0.625 & 0.125 & 0.875 \\
\hline 0.125 & 0.625 & 0.375 & 0.875 \\
\hline 0.125 & 0.375 & 0.625 & 0.875 \\
\hline 0.375 & 0.125 & 0.625 & 0.875 \\
\hline 0.375 & 0.625 & 0.125 & 0.875 \\
\hline 0.125 & 0.375 & 0.625 & 0.875 \\
\hline 0.125 & 0.375 & 0.625 & 0.875 \\
\hline 0.375 & 0.125 & 0.625 & 0.875 \\
\hline 0.375 & 0.625 & 0.125 & 0.875 \\
\hline 0.125 & 0.375 & 0.625 & 0.875 \\
\hline 0.375 & 0.125 & 0.625 & 0.875 \\
\hline 0.625 & 0.125 & 0.875 & 0.375 \\
\hline 0.375 & 0.625 & 0.125 & 0.875 \\
\hline & & & \\
\hline 0.625 & 0.375 & 0.875 & 0.125 \\
\hline
\end{tabular}

\begin{tabular}{|c|}
\hline $\begin{array}{c}\text { Destination } \\
\text { Node }\end{array}$ \\
\hline$i 1$ \\
\hline$i 2$ \\
\hline$i 3$ \\
\hline$i 4$ \\
\hline$i 5$ \\
\hline$i 6$ \\
\hline$i 7$ \\
\hline$i 8$ \\
\hline$i 9$ \\
\hline$i 10$ \\
\hline$i 11$ \\
\hline$i 12$ \\
\hline$i 13$ \\
\hline$i 14$ \\
\hline$i 15$ \\
\hline$i 16$ \\
\hline
\end{tabular}

\begin{tabular}{|c|c|c|c|}
\hline \multicolumn{4}{|c|}{ Measured Values } \\
\hline 01 & \multicolumn{1}{|c|}{02} & \multicolumn{1}{|c|}{03} & 04 \\
\hline 0.19609 & 0.38016 & 0.57985 & 0.87446 \\
\hline 0.37497 & 0.6284 & 0.12907 & 0.87595 \\
\hline 0.07441 & 0.62969 & 0.38442 & 0.85865 \\
\hline 0.11321 & 0.3769 & 0.63134 & 0.86334 \\
\hline 0.35767 & 0.12564 & 0.63656 & 0.87409 \\
\hline 0.37316 & 0.62495 & 0.12884 & 0.87389 \\
\hline 0.15333 & 0.35065 & 0.64172 & 0.86575 \\
\hline 0.10074 & 0.38006 & 0.62392 & 0.88595 \\
\hline 0.38057 & 0.11647 & 0.62529 & 0.87705 \\
\hline 0.37224 & 0.62286 & 0.12834 & 0.87282 \\
\hline 0.09919 & 0.37378 & 0.61651 & 0.89222 \\
\hline 0.35348 & 0.14553 & 0.63405 & 0.88816 \\
\hline 0.63863 & 0.09128 & 0.99357 & 0.36039 \\
\hline 0.38011 & 0.62046 & 0.11562 & 0.87847 \\
\hline 0.00142 & 0.00139 & 0.00143 & 0.0017 \\
\hline 0.61377 & 0.37812 & 0.99982 & 0.14173 \\
\hline
\end{tabular}
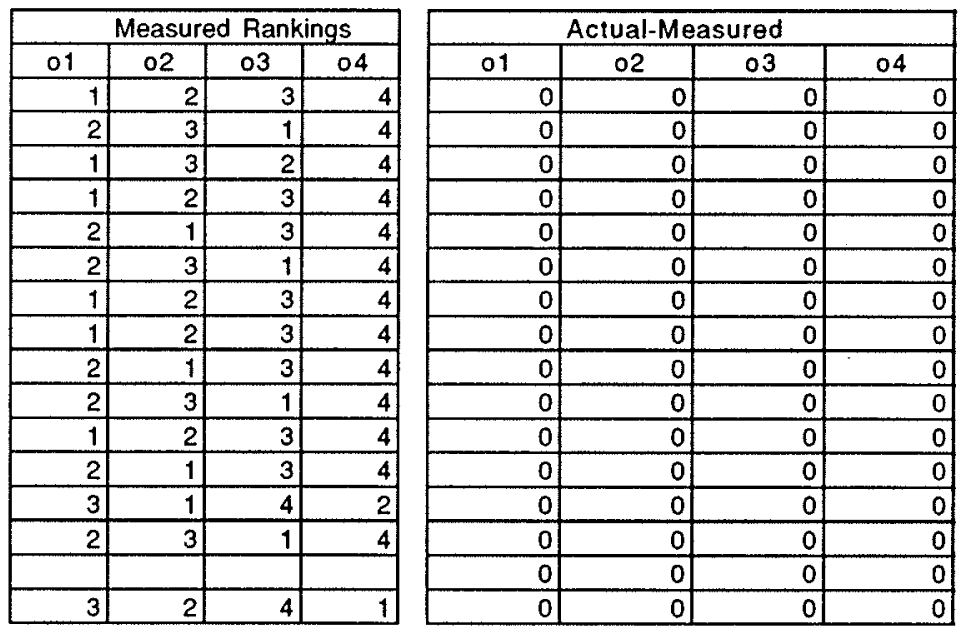

Page C.15 
Routing Results for Node 16

of the Sample Network

\begin{tabular}{|c|}
\hline $\begin{array}{c}\text { Destination } \\
\text { Node }\end{array}$ \\
\hline$i 1$ \\
\hline$i 2$ \\
\hline$i 3$ \\
\hline$i 4$ \\
\hline$i 5$ \\
\hline$i 6$ \\
\hline$i 7$ \\
\hline$i 8$ \\
\hline$i 9$ \\
\hline$i 10$ \\
\hline$i 11$ \\
\hline$i 12$ \\
\hline$i 13$ \\
\hline$i 14$ \\
\hline 115 \\
\hline$i 16$ \\
\hline
\end{tabular}

\begin{tabular}{|r|r|r|r|}
\hline \multicolumn{4}{|c|}{ Real Output Values } \\
\hline 01 & \multicolumn{1}{|c|}{02} & \multicolumn{1}{|c|}{03} & \multicolumn{1}{c|}{04} \\
\hline 0.6001 & 0.66277 & 0.7387 & 0.69211 \\
\hline 0.83195 & 0.68335 & 0.79365 & 0.65356 \\
\hline 0.7324 & 0.5838 & 0.6941 & 0.57503 \\
\hline 0.7421 & 0.52077 & 0.60967 & 0.55011 \\
\hline 0.7579 & 0.5601 & 0.5809 & 0.64446 \\
\hline 0.84136 & 0.57155 & 0.68184 & 0.52126 \\
\hline 0.84165 & 0.45933 & 0.56963 & 0.45056 \\
\hline 0.85577 & 0.4071 & 0.496 & 0.4857 \\
\hline 0.9459 & 0.3721 & 0.3929 & 0.45646 \\
\hline 1 & 0.45267 & 0.56297 & 0.43873 \\
\hline 0.95141 & 0.34957 & 0.45987 & 0.34079 \\
\hline 1 & 0.2307 & 0.341 & 0.31506 \\
\hline 1 & 0.3915 & 0.1802 & 0.47586 \\
\hline 1 & 0.44942 & 0.55972 & 0.31426 \\
\hline 1 & 0.34046 & 0.45076 & 0.2053 \\
\hline & & & \\
\hline
\end{tabular}

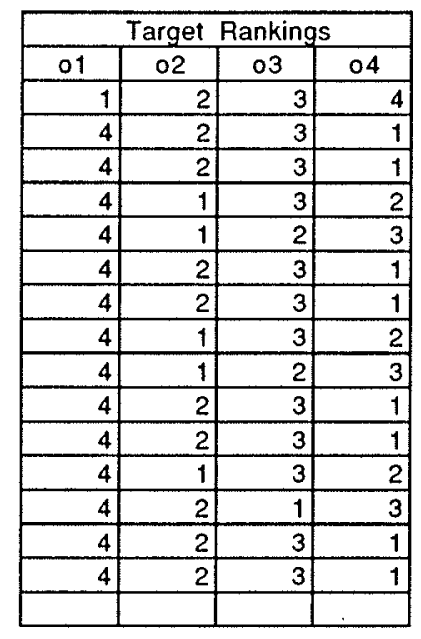

\begin{tabular}{|l|l|l|l|}
\hline \multicolumn{5}{|c|}{ Target Values } \\
\hline 01 & 02 & 03 & 04 \\
\hline 0.125 & 0.375 & 0.625 & 0.875 \\
\hline 0.875 & 0.375 & 0.625 & 0.125 \\
\hline 0.875 & 0.375 & 0.625 & 0.125 \\
\hline 0.875 & 0.125 & 0.625 & 0.375 \\
\hline 0.875 & 0.125 & 0.375 & 0.625 \\
\hline 0.875 & 0.375 & 0.625 & 0.125 \\
\hline 0.875 & 0.375 & 0.625 & 0.125 \\
\hline 0.875 & 0.125 & 0.625 & 0.375 \\
\hline 0.875 & 0.125 & 0.375 & 0.625 \\
\hline 0.875 & 0.375 & 0.625 & 0.125 \\
\hline 0.875 & 0.375 & 0.625 & 0.125 \\
\hline 0.875 & 0.125 & 0.625 & 0.375 \\
\hline 0.875 & 0.375 & 0.125 & 0.625 \\
\hline 0.875 & 0.375 & 0.625 & 0.125 \\
\hline 0.875 & 0.375 & 0.625 & 0.125 \\
\hline & & & \\
\hline
\end{tabular}

\begin{tabular}{|c|}
\hline $\begin{array}{c}\text { Destination } \\
\text { Node }\end{array}$ \\
\hline$i 1$ \\
\hline$i 2$ \\
\hline$i 3$ \\
\hline$i 4$ \\
\hline 15 \\
\hline$i 6$ \\
\hline$i 7$ \\
\hline$i 8$ \\
\hline$i 9$ \\
\hline$i 10$ \\
\hline$i 11$ \\
\hline$i 12$ \\
\hline$i 13$ \\
\hline$i 14$ \\
\hline$i 15$ \\
\hline$i 16$ \\
\hline
\end{tabular}

\begin{tabular}{|c|c|c|c|}
\hline \multicolumn{4}{|c|}{ Measured Values } \\
\hline 01 & 02 & 03 & 04 \\
\hline 0.12483 & 0.37529 & 0.62509 & 1 \\
\hline 0.86913 & 0.37058 & 0.62427 & 0.13071 \\
\hline 0.87751 & 0.38386 & 0.61433 & 0.09268 \\
\hline 0.87292 & 0.08957 & 0.63114 & 0.37628 \\
\hline 0.8794 & 0.11827 & 0.37875 & 0.62546 \\
\hline 0.86869 & 0.37344 & 0.62484 & 0.13219 \\
\hline 0.86987 & 0.36938 & 0.62723 & 0.13355 \\
\hline 0.8737 & 0.17935 & 0.59951 & 0.37171 \\
\hline 0.87727 & 0.12869 & 0.36769 & 0.62402 \\
\hline 0.87969 & 0.38996 & 0.61727 & 0.09295 \\
\hline 0.8848 & 0.32721 & 0.64823 & 0.1552 \\
\hline 0.87887 & 0.08978 & 0.63454 & 0.37573 \\
\hline 0.87083 & 0.37603 & 0.12861 & 0.62488 \\
\hline 0.86847 & 0.3733 & 0.62478 & 0.13374 \\
\hline 0.87825 & 0.37791 & 0.62333 & 0.10166 \\
\hline 0.00116 & 0.00099 & 0.00109 & 0.00102 \\
\hline
\end{tabular}
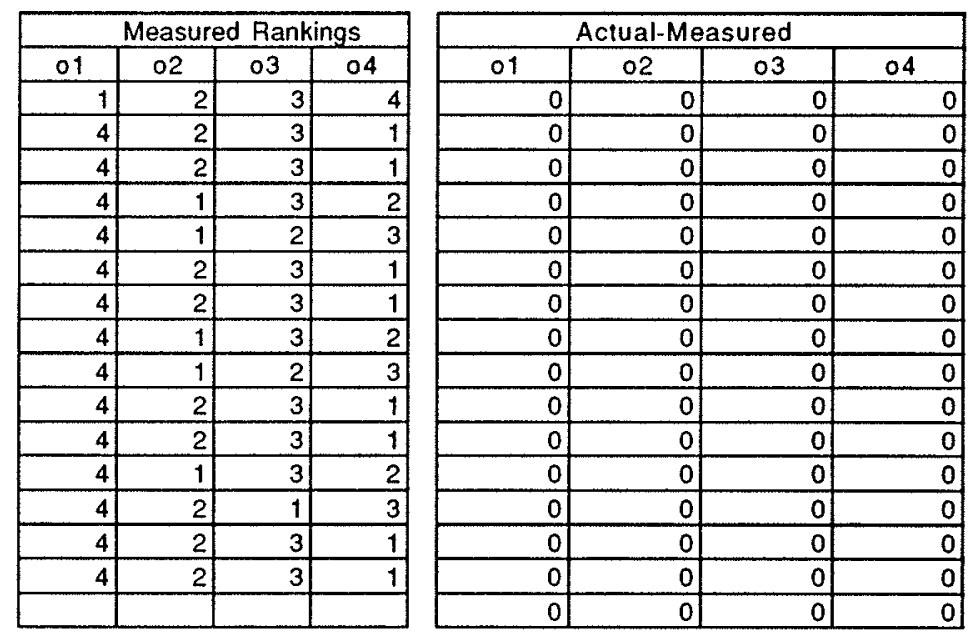

Page C.16 